\author{
Universidade de São Paulo \\ Instituto de Física
}

\title{
Mudanças de opinião em Redes Complexas
}

\author{
André Martin Timpanaro
}

Orientadora: Prof. $^{\text {a }}$ Dr. ${ }^{\text {a }}$ Carmen Pimentel Cintra do Prado

Tese de doutorado apresentada ao Instituto de Física para a obtenção do título de Doutor em Ciências

Comissão examinadora:

Prof. ${ }^{a}$ Dr. ${ }^{\text {a }}$ Carmen Pimentel Cintra do Prado (orientadora/IF-USP)

Prof. Dr. Iberê Luiz Caldas (IF-USP)

Prof. Dr. Eduardo Colli (IME-USP)

Prof. Dr. Marcus Aloizio Martinez de Aguiar (IFGW-UNICAMP)

Prof. Dr. Thierry Corrêa Petit Lobão (UFBA)

São Paulo

2012 


\section{FICHA CATALOGRÁFICA}

Preparada pelo Serviço de Biblioteca e Informação do Instituto de Física da Universidade de São Paulo

Timpanaro, André Martin

Mudanças de opinião em Redes Complexas.

São Paulo, 2012

Tese (Doutorado) - Universidade de São Paulo.

Instituto de Física

Orientadora: Prof $^{\mathrm{a}} \mathrm{Dr}^{\mathrm{a}}$ Carmen Pimentel Cintra do Prado

Área de Concentração:

Unitermos: 1. Dinâmica Estocástica; 2. Métodos de Perturbação; 3. Física Teórica; 4. Física Computacional. 



\title{
Agradecimentos
}

\author{
"I can no other answer make but thanks" \\ - William Shakespeare: Noite de Reis, ato III, cena III
}

Agradeço à minha orientadora, professora Carmen Pimentel Cintra do Prado, tanto pela liberdade com que ela permitiu que eu conduzisse esse trabalho - sem a qual ele seria radicalmente diferente - como por todas as conversas que tivemos, por todas às vezes que ela me trouxe os pés de volta ao chão e pela ajuda na hora de escrever artigos, ou resolver trâmites burocráticos.

Pelas diversas sugestões fornecidas, agradeço aos professores Nestor Felipe Caticha Alfonso, Domingos Humberto Urbano Marchetti e Thierry Corrêa Petit Lobão.

For the warm welcome I would like to thank the whole research group from the Institute of Complex Systems and Mathematical Biology of the University of Aberdeen, with special thanks going to Celso Grebogi, Alessandro P. S. Moura, Murilo da Silva Baptista, Marco Thiel, Naveed Kahn, Elahe Radmaneshfar, Jens Karschau and Luca Ciandrini. I would also like to thank my flatmates in Aberdeen, Rubens Dolmans and Mark Sangster, for making life much more fun on those two months.

For the help given to divulge this work I would like to thank professor Henrik Jeldtoft Jensen from the Imperial College in London and professor Katarzina Sznajd-Weron from the University of Wrocław.

Por discussões sobre topologia, que acabaram levando a uma simplificação crucial de alguns dos resultados, gostaria de agradecer à Caio Cicero Gomes.

Gostaria de agradecer pelas conversas desses últimos 5 anos, tenham sido elas acadêmicas ou não, aos colegas Maycon, Antônio Mário, Helder, Henrique, Átila, Fleury, Danilo, Abdo, Jozismar, Eduardo, Bruno, Bóris, Jonatas e Rafael.

Queria agradecer ao pessoal do Catadão-USP, principalmente o Raul, Rodrigo, Paulo, Tânia, Mayra, Hermínio, Escada, Feijão, Bia, Diana, Ferrero, Augusto, Aida, Renata e os nossos treinadores Renata, Guga, André, Okuda e Arthur. Cabe também um agradecimento especial, mas infelizmente póstumo ao Betão.

Agradeço aos meus amigos Elton, Daniel, Uêba, Rebeca, Chico, Dudu, Gabriel, Gabi, Leo, Siumara, Yoshi, Pirata, Fozzy, Caio, Manu, Wilson, Quel, Renato, Pivo, Elisa, Wilma, 
Juliana, Super, Mattos, Capeta, Erich, Lombardi e mais outros que por ventura tenham sido omitidos, pela convivência, todas as conversas e momentos de descontração.

Agradeço a Fundação de Amparo à Pesquisa do Estado de São Paulo, pelo apoio financeiro, sem o qual este trabalho não teria sido possível.

Finalmente, agradeço a minha família, Wagner, Rita, Julian e Madalena por todo o apoio que me deram ao longo de todo esse período. 


\section{Resumo}

Nos últimos anos, uma míriade de modelos de propagação de opinião foram propostos, motivados pelo interesse crescente dos físicos por problemas interdisciplinares tanto em sociologia, quanto em economia e biologia. Um dos objetivos desse trabalho é unificar alguns desses modelos em uma mesma formulação. Para isso, generalizamos a noção de confiança limitada para o que chamamos de regras de confiança, que podem ser interpretadas como a introdução de viéses ou preconceitos nas interações de agentes com opiniões distintas. Munidos dessa formulação, nos propusemos a estudar como modelos que promovem localmente conformidade (o que está de acordo com experimentos para grupos pequenos conduzidos por psicólogos), poderiam gerar diversidade globalmente (explicando a persistência de pontos de vista distintos em sociedades, por exemplo). Nós estudamos o campo médio do modelo do votante e de variantes do modelo Sznajd. Aplicando ferramentas de sistemas dinâmicos, conseguimos resolver analiticamente o comportamento qualitativo dos modelos na ausência de ruído e desenvolvemos uma teoria de perturbação para o modelo Sznajd com ruído infinitesimal, que nos forneceu um retrato parcial do comportamento na presença de ruído. $\mathrm{Na}$ ausência de ruído, chegamos a conclusão que o modelo do votante se comporta de maneira completamente diferente, enquanto que os outros modelos tem essencialmente o mesmo comportamento. Também fizemos simulações em redes Barabási-Albert e Watts-Strogatz para os modelos votante e Sznajd e, em colaboração com o grupo de pesquisa do Institute for Complex Systems and Mathematical Biology da Universidade de Aberdeen, estudamos um modelo de biodiversidade que pode ser encarado como uma variante do modelo do votante em uma rede quadrada. As nossas conclusões apontam que os resultados de campo médio podem ser compreendidos através de conexões com teoria de grafos e que os diversos modelos simulados se comportam em um certo sentido da mesma maneira, reforçando a idéia de universalidade entre eles (na verdade é essencial que existam aspectos universais no comportamento humano para que a modelagem de sistemas sociais seja factível, dadas as dificuldades óbvias de se construir um modelo realista para uma pessoa ou uma sociedade). Grosso modo, em todos os sistemas estudados, a coexistência ou não de pontos de vista diferentes parece depender mais crucialmente da rede e do tipo de regra de confiança, do que de outros detalhes específicos do modelo. 



\section{Abstract}

In the recent years, a great number of opinion propagation models were proposed, motivated by the increasing interest among physicists in interdisciplinary problems, not only in sociology, but also in economics and biology. One of the goals of this work is to unify some of these models under a same formulation. In order to do that, we generalized the notion of bounded confidence to what we called confidence rules, that can be interpreted as the introduction of biases and prejudices in the interactions among agents holding differing points of view. Using this formulation, we decided to study how models that locally breed conformity (what is in accordance with experiments conducted by psichologists for small groups) could sustain diversity globally (explaining the persistence of different points of view in societies, for example). We studied the mean field version of the voter model and of variants of the Sznajd model. We used dynamical systems techniques and were able to solve analytically the qualitative behaviour of the models in the absence of noise and developed a perturbation theory for the Sznajd model with infinitesimal noise, that yielded a partial picture of the behaviour with noise. In the absence of noise, we found that the voter model has a completely different behaviour, while the other models have essentially the same behaviour. We also did simulations in Barabási-Albert and Watts-Strogatz networks for the voter and the Sznajd models and we collaborated with the research group of the Institute for Complex Systems and Mathematical Biology from the University of Aberdeen, studying a biodiversity model that can be seen as a modification of the voter model in a square lattice. Our conclusions point that the mean field results can be understood through connections with graph theory problems and that the different models that were simulated, in some sense, have the same behaviour, reinforcing the idea of universality for these models (due to the obvious difficulties in modelling human beings in a reliable and realistic way, some degree of universality in human behaviour is actually essential, in order for social modelling to be feasible). Roughly speaking, in all the systems that were studied, the coexistence or not of differing opinions, seems to depend more strongly on the network and on the type of confidence rule used, than in other specific details of the model. 



\section{Sumário}

$\begin{array}{lll}\text { Agradecimentos } & \text { v }\end{array}$

Resumo vii

$\begin{array}{ll}\text { Abstract } & \text { ix }\end{array}$

1 Introdução 1

1.1 Sociofísica e propagação de opiniões . . . . . . . . . . . . . . . . . 1

1.2 Alguns conceitos de teoria de grafos . . . . . . . . . . . . . . . 7

1.2.1 Redes complexas e redes sociais . . . . . . . . . . . . . . 13

1.3 Conceitos de sistemas dinâmicos . . . . . . . . . . . . . . . . . . . 18

2 Definições dos modelos 23

2.1 Modelagem com redes complexas e regras de confiança . . . . . . . . . . . 23

2.2 Convencimento, Adaptação e Homogeneização . . . . . . . . . . . . . . . . . . . . . . . . . 25

2.3 O Modelo do Votante e o modelo Ochrombel . . . . . . . . . . . . . 25

2.4 O modelo Sznajd . . . . . . . . . . . . . . . . . . . . 27

2.5 O modelo $q$-votante e variações do modelo Sznajd . . . . . . . . . . . . . . 29

2.6 O Modelo Deffuant e o modelo HK . . . . . . . . . . . . . . . . . 30

2.7 O modelo Axelrod . . . . . . . . . . . . . . . . 31

3 Dedução das equações de campo médio a partir de cadeias de Markov 33

3.1 Um exemplo simples de cadeia de Markov . . . . . . . . . . . . . . . 33

3.2 Notações . . . . . . . . . . . . . . . . . . . . . . 35

3.3 O modelo do votante . . . . . . . . . . . . . . 36

3.4 O modelo Ochrombel . . . . . . . . . . . . . . . . . . . . . 41

3.5 O modelo Sznajd . . . . . . . . . . . . . . . . . . . 43

3.6 Observações sobre essas deduções . . . . . . . . . . . . . . . . 47

4 Resultados Analíticos para a aproximação de campo médio 49

4.1 Considerações iniciais . . . . . . . . . . . . . . . . 50 
4.2 Pontos fixos e estabilidade . . . . . . . . . . . . . . . . . 51

4.2.1 Regras de confiança com mais de uma componente . . . . . . . . 53

4.2 .2 Pontos fixos de coexistência . . . . . . . . . . . . . . 56

4.2.3 Pontos fixos em que opiniões são extintas . . . . . . . . . . . . . . 58

4.3 Existência dos pontos fixos . . . . . . . . . . . . . 63

4.4 O modelo Sznajd com ruído . . . . . . . . . . . . . . . . . . 68

4.4.1 Modelo usual com ruído uniforme . . . . . . . . . . . . . . . . 69

4.4.2 O modelo com regras de confiança e ruído infinitesimal . . . . . . . 71

4.5 Generalizações do modelo Sznajd . . . . . . . . . . . . . . . . . . . 94

4.6 O modelo do votante . . . . . . . . . . . . . . . . . . . 99

5 Resultados de Simulação $\quad 103$

5.1 Atratores e Estabilidade . . . . . . . . . . . . . . . . . . . . . . . . . . . . 104

5.2 Opiniões inertes sobreviventes . . . . . . . . . . . . . . . 107

5.3 Regras sem atratores estacionários . . . . . . . . . . . . . . . . . 108

5.4 Transientes Longos e Estados Estacionários . . . . . . . . . . . . . . . . . 109

5.5 Simulações em redes de Watts-Strogatz e competição cíclica . . . . . . . . 111

5.6 Comparações quantitativas para o caso de 3 opiniões . . . . . . . . . . . . . 117

5.7 Modelo com regras de confiança e ruído . . . . . . . . . . . . . . . . 121

5.8 O modelo do votante . . . . . . . . . . . . . . . . . . . 122

5.9 O modelo rock-paper-scissors de biodiversidade . . . . . . . . . . . . 128

6 Conclusão 131

A Teoremas utilizados $\quad 139$

A.1 Teoremas sobre grafos . . . . . . . . . . . . . . . . . . . 140

A.2 Teoremas sobre matrizes . . . . . . . . . . . . . . . . 142

A.3 Derivadas de autovalores e teoria de perturbação em matrizes . . . . . . . . 153

A.4 Teoremas de Topologia e de Sistemas Dinâmicos . . . . . . . . . . . . . 154

B Códigos utilizados $\quad 157$

B.1 Formatos dos arquivos utilizados . . . . . . . . . . . . . . 158

B.1.1 Arquivos de regra de confiança . . . . . . . . . . . . . 158

B.1.2 Arquivos de geometria . . . . . . . . . . . . . . . . 158

B.1.3 Arquivos de condição inicial e de saída (estado microscópico) . . . 159

B.1.4 Arquivos de saída (estado macroscópico) . . . . . . . . . . . . . . 159

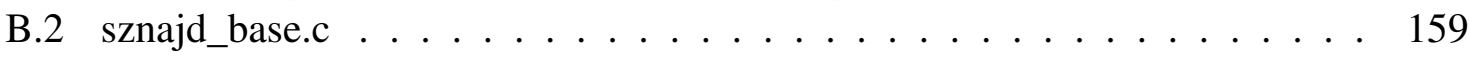

B.2.1 Adaptações para o modelo do votante . . . . . . . . . . . . 170

B.3 cria_BA.c ........................ 171

$\begin{array}{ll}\text { Referências Bibliográficas } & 175\end{array}$ 


\section{Lista de Figuras}

1.2.1 Representação de um grafo . . . . . . . . . . . . . . . . . . 8

1.2.2 Um grafo ponderado e os seus esqueletos, direcionado e não-direcionado. 9

1.2.3 Exemplo de grafo reverso . . . . . . . . . . . . . . . . . . . . . 9

1.2.4 Exemplo de fontes e sorvedouros . . . . . . . . . . . . . . . . . . . . 10

1.2.5 Exemplo de subgrafo induzido . . . . . . . . . . . . . . . . . . . . 11

1.2.6 Exemplo de grafo completo . . . . . . . . . . . . . . . . . . . . . . . . . . . . . . . . . . .

1.2.7 Exemplo de clique . . . . . . . . . . . . . . . . . . . . . . . . . . . . . . . . . 12

1.2.8 Exemplo de união de grafos . . . . . . . . . . . . . . . . 13

2.1.1 Representação de uma regra de confiança . . . . . . . . . . . . . . . 24

4.4.1 Contra-exemplo para o espectro da matriz de perturbação . . . . . . . . . . 94

5.1 .1 Uma regra $\mathcal{R}$ com 2 atratores . . . . . . . . . . . . . . . 104

5.1.2 Séries temporais mostrando os atratores da dinâmica para $\mathcal{R}$. . . . . . . 105

5.1.3 Projeção no espaço de fase para as trajetórias da regra $\mathcal{R}$. . . . . . . . . . 106

5.2.12 regras $\left(\mathcal{R}_{1}\right.$ e $\left.\mathcal{R}_{2}\right)$ em que opiniões inertes conseguem sobreviver . . . . . 107

5.2.2 Séries temporais para as regras $\mathcal{R}_{1}$ e $\mathcal{R}_{2}$ (primeira parte) . . . . . . . . 107

5.2.2 Séries temporais para as regras $\mathcal{R}_{1}$ e $\mathcal{R}_{2}$ (segunda parte) . . . . . . . . 108

5.3 .1 Uma regra sem atratores . . . . . . . . . . . . . . . . 108

5.4.1 Uma regra com predisposição a transientes longos e estados congelados . . 110

5.4.2 Gráfico de tempo de retorno, mostrando os transientes longos . . . . . . . . 111

5.5 .1 A regra de 2 partidos . . . . . . . . . . . . . . . . . . . . . 112

5.5.2 Séries temporais mostrando o regime oscilatório . . . . . . . . . . . . . 113

5.5.3 Amplitude das oscilações e porcentagem de simulações que atingem o estado estacionário . . . . . . . . . . . . . . . . . . . . 114

5.5.4 Projeções no espaço de fase mostrando o regime oscilatório para redes WS e sua ausência em redes BA . . . . . . . . . . . . . . . . . . . 115

5.5.5 Porcentagem das simulações que exibem oscilações para diferentes regras 116

5.6.1 Espaço de fase para o modelo com 3 opiniões e confiança limitada . . . . . 118

5.6.2 Detalhe do espaço de fase em torno da condição inicial uniforme . . . . . . 118 
5.6.3 Espaços de fase para 3 opiniões (primeira parte) . . . . . . . . . . . . 119

5.6.3 Espaços de fase para 3 opiniões (segunda parte) . . . . . . . . . . . . . . 120

5.7.1 Regra de confiança do exemplo da teoria de perturbação . . . . . . . . . . . 121

5.7.2 Espaço de fase exemplificando o modelo no campo médio com ruído . . . . 122

5.8.1 Espaço de fase para o modelo do votante, em um caso com 3 opiniões . . . 123

5.8.2 Detalhe do espaço de fase . . . . . . . . . . . . . . . . . . . . . 124

5.8.3 Série temporal na rede da grandeza conservada do campo médio . . . . . . 124

5.8.4 Espaço de fase para o modelo do votante, em um caso com 4 opiniões . . . 126

5.8.5 Série temporal das grandezas conservadas do campo médio . . . . . . . . . 127

5.9.1 Padrões espaciais exibidos pelo modelo RPS, para diferentes valores de assimetria . . . . . . . . . . . . . . . 130 


\section{Capítulo 1}

\section{Introdução}

"A theory that you can't explain to a bartender is probably no damn good."

- Ernest Rutherford

"Men build too many walls and not enough bridges."

- atribuído a Newton por Georges Pire

\subsection{Sociofísica e propagação de opiniões}

A sociofísica é uma área que surgiu nos últimos anos em virtude de esforços para aplicar ferramentas típicas da física na compreensão de sistemas sociais. Esses esforços são em grande parte consequência do interesse crescente entre os físicos por problemas interdisciplinares, como problemas provenientes da biologia e da economia, e o potencial das ferramentas comumente usadas na física para resolver esses problemas. A maior parte desses esforços diz respeito aos sistemas complexos, que são sistemas com muitos componentes que exibem um comportamento coletivo que não é óbvio a partir do comportamento de cada uma de suas partes (esses comportamentos são denominados emergentes na literatura). Sistemas desse tipo costumam exibir estruturas hierárquicas (que às vezes não são óbvias) e interações que não são homogêneas; alguns exemplos são sociedades, redes metabólicas, ecossistemas, mercados financeiros, etc. A intenção nesses esforços interdisciplinares é a de entender quais seriam os ingredientes essenciais do comportamento dos componentes do sistema para que um determinado comportamento emergente surja, de forma que a modelagem desses sistemas é fortemente influenciada pela idéia de universalidade. Quase sempre, modelos extremamente simplificados são utilizados e os resultados dependem, quase que exclusivamente, de simulações em computador (uma das razões para o aumento no interesse é que o aumento no poder de processamento dos computadores atuais torna cada vez mais factível o estudo desses modelos). Um trabalho de revisão da literatura dessa área foi realizado recentemente ${ }^{[8]}$. 
A idéia de modelar matematicamente a sociedade é muito mais antiga do que os esforços recentes, ou mesmo que dos computadores que tornaram esses estudos possíveis e remonta aos estudos de Adolphe Quetelet sobre estatísticas sociais, de Auguste Comte, que tentou aplicar diretamente leis da física nas ciências sociais, e da criação da sociologia como uma disciplina embasada na estatística, por Émile Durkheim. Os esforços mais recentes remontam ao desenvolvimento da sociodinâmica ${ }^{[48]}$, ao modelo de Schelling ${ }^{[33]}$ e aos trabalhos de Serge Galam ${ }^{[18]}$ e Robert Axelrod ${ }^{[2]}$.

Os modelos que serão estudados nesse trabalho são os modelos de propagação de opiniões. Em um modelo de propagação de opiniões estamos modelando uma sociedade, constituída de agentes que possuem opiniões e essas opiniões se alteram através de interações entre esses agentes. Alguns desses modelos são definidos em uma rede social, ou seja em um grafo que define quais agentes interagem com quais. Nesse caso, são necessários resultados experimentais. Redes sociais são um exemplo de rede complexa, um tipo de rede que começou a ser estudado na década de 90 e que parece estar presente em vários aspectos do nosso dia a dia (mais detalhes sobre redes complexas se encontram mais adiante na seção 1.2.1. Também recomendamos a leitura de um trabalho de revisão sobre esse assunto ${ }^{[5]}$ ). Por essa razão, apesar de estudos usando redes clássicas como reticulados, ainda serem comuns, modelos provenientes do estudo de redes complexas, ou mesmo redes complexas obtidas experimentalmente, são usadas como substrato em modelos de propagação de opinião. Para que a modelagem seja séria, também é necessário que as regras desses modelos, sobre como as interações entre os agentes se dão, tenham alguma fundamentação experimental. No caso da propagação de opiniões, esses experimentos são provenientes da psicologia. Podemos citar como influentes, os experimentos de $\mathrm{Asch}^{[1]}$ sobre conformidade, o experimento de Sherif ${ }^{[36]}$ sobre o efeito autocinético e as idéias de Festinger ${ }^{[14]}$ sobre dissonância cognitiva (refinadas em trabalhos mais recentes ${ }^{[13 ; 28]}$ ).

A complexidade dos seres humanos implica que qualquer modelagem deve ser uma aproximação grosseira, de forma que só é factível encarar esses resultados experimentais, sobre como pessoas interagem entre si, como um guia de como as regras devem se parecer qualitativamente. Não há a menor dúvida que os modelos que são usados nessa área não tem regras microscópicas que sejam acuradas, porém se o comportamento humano tiver aspectos universais, então as minúcias dos modelos não devem ser importantes e esperamos que existam classes de modelos, em que se fizermos pequenas modificações nas regras, não devemos ter modificações do comportamento geral do sistema. Mais do que isso, esses aspectos universais são cruciais. Se eles não existissem, então somente modelos extremamente realistas sobre pessoas teriam alguma chance de descrever a sociedade, mesmo se nos restringirmos aos comportamentos qualitativos, o que significaria efetivamente que o problema se encontraria fora tanto do alcance computacional atual, quanto do conhecimento atual de psicologia (que não alcança esse nível de detalhamento).

Dado que o caráter universal do comportamento humano seria um componente essencial, então bastaria procurar classes de modelos que tivessem o mesmo comportamento e tentar 
encontrar quais são os seus componentes essenciais (de forma semelhante ao que acontece com modelos de mecânica estatística e as simetrias que eles obedecem em um nível microscópico). Porém o que se observa na literatura é que não existe essa preocupação. O caráter qualitativo das regras, segundo as quais os agentes interagem, torna difícil impor restrições fortes na forma que essas regras podem ter e, como consequência, existe na literatura um grande número de modelos de propagação de opinião, que são na verdade pequenas variações de alguns poucos modelos. As formas como as modificações dos modelos são geralmente introduzidas não levam em consideração a possibilidade de comparações simples entre elas, o que faz com que a literatura seja bastante fragmentada e não sejam claros os efeitos que cada modificação causa. Além disso, poucos trabalhos analisam cada modificação e muitas acabam abandonadas rapidamente.

A situação é complicada também por dois outros fatores: a maior parte dos resultados obtidos nesses modelos dependem quase que exclusivamente de simulações computacionais, com poucos resultados analíticos (apesar disso ser um problema no estudo de sistemas complexos em geral, não apenas dos modelos de propagação de opiniões) e a confirmação experimental de resultados dessa área e de outras áreas relacionadas da sociofísica (que não são propriamente áreas diferentes, já que elas só diferem quanto a aspectos de interpretação) ainda é preliminar, com resultados de eleições ainda sendo a maior fonte de dados. Além disso, o aspecto qualitativo dos modelos significa que qualquer tentativa de fazer uma confirmação experimental deve ser cuidadosa. As únicas previsões que fazem sentido de serem testadas experimentalmente são ou macroscópicas ou qualitativas. Isso quer dizer que as únicas previsões possíveis, nesses modelos, dizem respeito a coisas como a distribuição de votos de um candidato, quando um grupo atinge uma situação de consenso, condições em que um idioma pode se subdividir e outras previsões estatísticas como estas.

De um ponto de vista matemático, esses modelos são em sua maioria baseados no modelo de Ising, no modelo de Potts ou em modelos de difusão (as definições exatas de alguns modelos se encontram mais adiante, no capítulo 2). A maior parte dos estudos usam apenas 2 opiniões, representando uma escolha binária, como sim ou não, com apenas alguns usando opiniões contínuas, ou então, mais do que 2 opiniões discretas. Grosso modo, esses modelos exibem ou situações de consenso (ou seja, o modelo atinge uma situação em que todos os agentes concordam entre si e o estado do sistema para de evoluir) ou coexistência inativa de diferentes opiniões (não há consenso entre os agentes, porém as regras de evolução do modelo implicam que nada mais deve mudar), o que ocorre principalmente quando impomos que algumas opiniões não interagem entre si. Enquanto os resultados experimentais provenientes da psicologia, a que já nos referimos, sugerem que grupos pequenos de pessoas tendem a exibir situações de consenso, a experiência cotidiana diz que esse não é o caso para a sociedade como um todo. Diferentes pontos de vista persistem e além disso, a sociedade não é uma coisa estática. Dados os resultados dos modelos existentes na literatura, podemos levantar a seguinte pergunta:

Como explicar, através de um modelo simples, que gere consenso localmente, a persis- 
tência de uma coexistência ativa de opiniões distintas, na sociedade como um todo?

Uma solução possível seria um ruído térmico, porém nos modelos da literatura só conseguimos coexistências ativas em que mais de uma opinião é adotada por uma proporção considerável de agentes em situações em que a intensidade desse ruído térmico é grande. Ou seja, a coexistência surge porque o ruído passa a ser mais importante do que as interações internas do sistema. Essa situação pode ser interpretada como um sistema governado principalmente por influências externas. Por outro lado, pensando na interpretação desse ruído, vemos que a sua origem deve ser a própria sociedade (coisas como propaganda, ou um zeitgeist). Olhando por esse ponto de vista, se um ruído com intensidade alta é necessário, isso não significa que resolvemos o problema e sim, que algum ingrediente essencial foi deixado de fora no modelo original (sem ruído) e que esse modelo deve ser modificado. Apesar disso, um ruído térmico de intensidade pequena poderia modelar hesitação, ou mesmo individualidade, de forma que o papel do ruído não deve ser completamente desprezado, apenas que as coexistências deveriam persistir sem que o ruído precisasse dominar todas as outras interações.

O trabalho que desenvolvemos nesse Doutorado tem como motivação ajudar a sanar algumas dessas deficiências da literatura e avançar na compreensão da diversidade de opiniões que observamos na sociedade. Nós definimos um conjunto extremamente genérico de modificações, que chamamos de regras de confiança, que permite a abordagem de classes de modelos, ao invés de modelos isolados. Grosso modo, essa modificação diz que se um agente muda de opinião segundo as regras do modelo, então essa modificação deve acontecer com uma probabilidade que depende das opiniões envolvidas. Dessa forma, as regras de confiança podem ser interpretadas como uma modelagem de viéses e de preconceitos. Estudamos uma classe de modelos baseada no modelo Sznajd (definida no capítulo 2), em que grupos de agentes que concordam entre si tem um maior poder de convencimento (o que gera consenso localmente). Fizemos uma aproximação de campo médio para esses modelos, na presença de regras de confiança e usando ferramentas da teoria de sistemas dinâmicos, fomos capazes de mostrar que todos os modelos da classe estudada tem o mesmo comportamento. Além disso, esses comportamentos podem ser expressos de maneira bem simples, fazendo uma conexão com a teoria de grafos. Fizemos simulações desses modelos, comparando com os resultados obtidos no campo médio e discutimos brevemente a influência da rede social usada (utilizamos apenas redes Barabási-Albert e redes Watts-Strogatz, que serão definidas na seção 1.2.1). Finalmente, estudamos o que acontece com a adição de um ruído térmico pequeno na aproximação de campo médio. Os resultados mais importantes deste trabalho são os seguintes:

- O comportamento dos modelos na aproximação de campo médio depende apenas de alguns poucos aspectos qualitativos da regra de confiança. Mais precisamente, eles dependem apenas de quais opiniões são capazes de convencer quais opiniões. Isso sugere que é possível tratar modelos de sociofísica em termos de classes de modelos, ao contrário do que acontece hoje na literatura. 
- Os atratores e as estruturas observados na aproximação de campo médio só podem exibir consenso ou coexistências inativas, na ausência de ruído, explicando por que esses estados estacionários são tão prevalentes na literatura. Por outro lado, mesmo na presença de um ruído infinitesimal, estados estacionários diferentes passam a ser possíveis.

- Encontramos uma classe de regras de confiança, que quando simulada na rede WattsStrogatz exibe coexistência ativa mesmo na ausência de um ruído térmico, o que é um avanço na compreensão da existência de diversidade de opiniões na sociedade.

- Podemos fazer conjecturas sobre quais são os aspectos das interações sociais que são mais importantes para a emergência de diversidade de opiniões. Em particular, essas previsões são testáveis experimentalmente. Também podemos sugerir modificações não convencionais nesses modelos, que parecem ser promissoras.

Salientamos também que os resultados que obtivemos para o campo médio são analíticos, o que é algo escasso na literatura. Na nossa abordagem usando sistemas dinâmicos também é crucial o estudo dos efeitos que as condições iniciais podem ter no sistema (o que permitiu resolver inclusive uma controvérsia na literatura ${ }^{[43]}$ ). Outro aspecto incomum é que usamos mais do que 2 opiniões nesse trabalho. A maior parte dos trabalhos, por simplicidade, usa apenas duas opiniões, motivados por escolhas do tipo sim ou não. Nossos resultados mostram que pode ser útil considerar mais de 2 opiniões, mesmo que elas sejam apenas interpretadas como nuances de sim ou não, uma vez que as regras de confiança com apenas 2 opiniões são limitadas demais para exibirem comportamentos novos.

No resto dessa introdução apresentamos alguns conceitos de teoria de grafos (seção 1.2), que serão importantes na hora de expressar os resultados do campo médio. Essa seção se preocupa principalmente com definições básicas, nomenclatura e notação, junto com uma parte sobre redes complexas, em que definimos as redes de Watts-Strogatz e Barabási-Albert, assim como alguns dos índices que são comumente usados para caracterizar as redes complexas. A última seção dessa introdução (seção 1.3) apresenta de maneira resumida os fundamentos da análise de estabilidade linear, que é a principal ferramenta que vamos usar para analisar quais são os atratores do sistema. Em particular, explicamos o que acontece quando temos grandezas conservadas, o que não é normalmente discutido em livros-texto sobre o assunto. No capítulo 2 introduzimos de maneira mais precisa as regras de confiança e a forma como elas podem ser esquematizadas, usando grafos ponderados. Também falamos dos mecanismos mais presentes nos modelos de propagação da literatura e introduzimos as definições dos principais modelos que existem, juntamente com seus principais resultados. No capítulo 3 fazemos a dedução das equações de campo médio através de cadeias de Markov. Começamos com o movimento Browniano em uma dimensão, para fixar as idéias e depois introduzimos uma notação mais adequada pra tratar um modelo definido em uma rede. Fazemos essa dedução primeiro para o modelo do votante, depois para o modelo Ochrombel e finalmente para o modelo Sznajd (esses modelos podem ser encarados como 
complicações sucessivas). Salientamos que, até onde sabemos, esses cálculos são inéditos, apesar do resultado para o modelo do votante já ser conhecido, podendo ser obtido por meios mais simples. O capítulo 4 contém os resultados analíticos para o modelo estudado na aproximação de campo médio. Nesse capítulo, determinamos quais são os pontos fixos possíveis do modelo Sznajd, quais as suas estabilidades e mostramos a conexão entre essas propriedades e problemas tradicionais da teoria de grafos. Também resolvemos o modelo Sznajd com um ruído térmico na ausência de regras de confiança (esse problema já havia sido resolvido parcialmente ${ }^{[46]}$, mas a solução completa que apresentamos aqui ainda não foi publicada) e depois montamos uma teoria de perturbação para tratar o modelo com regras de confiança, o que é possível quando o ruído é infinitesimal. Finalmente, nós mostramos o que acontece quando aplicamos essa abordagem de sistemas dinâmicos para o modelo do votante e para uma variante do modelo Sznajd que tem uma ligação com o modelo $q$-votante. O capítulo 5 é onde estão apresentados os resultados de simulação. Não é essencial para a compreensão dos resultados de simulação a leitura dos capítulos 3 e 4. As simulações do modelo Sznajd sem ruído (seções 5.1 a 5.6) podem ser compreendidas apenas com a leitura do resumo dos resultados analíticos para esse caso, que se encontra na página 132 e da seção 1.2 para o leitor que não esteja familiriazado com as notações. Para a compreensão da seção 5.8 é necessária apenas a leitura da seção 4.6. O objetivo das simulações do modelo Sznajd que apresentamos aqui é mostrar como aparecem de fato os diversos resultados que obtivemos no campo médio e também dar uma idéia do limite de validade da aproximação de campo médio. A seção 5.1 mostra um exemplo em que todas as estruturas principais, previstas pelo campo médio, estão presentes e podem ser facilmente identificadas no espaço de fase. Na seção 5.2 mostramos situações, previstas pelo campo médio, em que opiniões que não conseguem convencer nenhuma outra são mesmo assim capazes de sobreviver no estado estacionário. A seção 5.3 comenta as regras sem atratores. Na seção 5.4 mostramos alguns resultados em que temos um desacordo entre os resultados da simulação e da aproximação de campo médio. A seção 5.5 trata das simulações na rede de Watts-Strogatz (todas as outras foram realizadas em redes Barabási-Albert), em um caso em que temos uma dominância cíclica entre as opiniões e onde conseguimos observar, para alguns valores dos parâmetros usados, uma coexistência ativa de todas as opiniões. A seção 5.6 mostra simulações para todos os casos (de um ponto de vista qualitativo) de 3 opiniões, comparando o campo médio e as simulações na rede Barabási-Albert (também comentamos uma controvérsia que existia para o caso com 3 opiniões e confiança limitada, que pudemos resolver ao olhar o comportamento no espaço de fase como um todo ${ }^{[43]}$ ). A seção 5.7 faz uma confirmação experimental da teoria de perturbação desenvolvida na seção 4.4.2. Na seção 5.8 apresentamos simulações do modelo do votante na rede Barabási-Albert, comparando com a integração das equações de campo médio. A seção 5.9 é independente do resto do trabalho. Nela nós fazemos um paralelo entre modelos de propagação de opinião e um modelo de biodiversidade conhecido como rock-paper-scissors (essa foi a parte do trabalho realizada na Universidade de Aberdeen). O capítulo 6 apresenta nossas conclusões, com uma recapitulação dos resultados que 
obtivemos, assim como alguns aspectos problemáticos desses resultados e possíveis soluções. Nos apêndices se encontram as provas dos teoremas utilizados e os códigos principais utilizados, para fins de referência.

\subsection{Alguns conceitos de teoria de grafos}

Um dos pontos importantes desse trabalho são as conexões que existem entre os resultados qualitativos dos modelos estudados com problemas tradicionais de teoria de grafos. Além disso, como os modelos são definidos em redes complexas (que nada mais são do que uma classe de grafos com certas propriedades especiais), apresentamos nessa seção uma introdução a teoria de grafos, que apesar de não ser completamente informal não tem também a pretensão de ser rigorosa, nem exaustiva. No apêndice A.1 podem ser encontrados conceitos menos elementares sobre grafos, assim como alguns teoremas que nós provamos sobre esse assunto.

Um grafo (usaremos nesse texto grafo e rede como sinônimos) é uma representação abstrata de um conjunto de relações entre objetos quaisquer. Formalmente, um grafo $G$ é definido por um conjunto de sítios (sítios, nós e vértices são usados como sinônimos) $V \neq \varnothing$ (os objetos que estão sendo considerados) e alguma estrutura que estabelece relações entre os elementos de $V$. O tipo de estrutura reflete obviamente propriedades diferentes dessas relações e determina qual será o tipo de grafo. Além disso, traduzindo uma estrutura de um tipo para outro é possível estabelecer conexões entre os diferentes tipos de grafos. As 3 formas de estabelecer relações entre vértices que iremos usar são arestas, arcos e pesos, que definem respectivamente grafos não-direcionados, grafos direcionados e grafos ponderados. Uma aresta é um conjunto com 2 nós distintos e denota uma relação que é simétrica entre os 2 nós pertencentes ao conjunto, um arco é um par ordenado com 2 nós distintos e denota relações que podem exibir assimetrias e o peso de um arco ou de uma aresta é um número real associado a um arco ou a uma aresta que tipicamente denota a intensidade de uma interação ou distâncias em algumas aplicações, sendo em geral uma forma de definir relações que não são homogêneas. Dessa forma, podemos definir um grafo não-direcionado como um par $(V, E)$ onde $E$ é um conjunto de arestas, um grafo direcionado como um par $(V, A)$ onde $A$ é um conjunto de arcos e um grafo ponderado pode ser definido por uma função $p$ de $V^{2}$ em $\mathbb{R}$, onde $p(i, j)$ é o peso do arco $(i, j)$ (ou aresta $\{i, j\}$ se $p(i, j)=p(j, i)$ ). Se o conjunto $V$ é finito pode-se colocar os pesos em uma matriz, para obter a chamada matriz de adjacências.

Dado um grafo $G$ qualquer, vamos sempre nos referir ao conjunto de vértices como $V(G)$, o conjunto de arestas como $E(G)$, o conjunto de arcos como $A(G)$ e a sua matriz de adjacências como $M(G)$ (os termos da matriz de adjacências serão denotados por $M_{i \rightarrow j}$ ). Se o grafo for finito, o número de elementos de $V(G)$ será chamado de tamanho do grafo, $N(G)$. Pode-se atribuir a uma aresta $\{i, j\} 2 \operatorname{arcos}(i, j)$ e $(j, i)$. Da mesma forma podese atribuir a um arco $(i, j)$ a aresta $\{i, j\}$. Finalmente se um grafo não é ponderado pode-se 
mesmo assim falar da sua matriz de adjacências, atribuindo peso 1 a todos os arcos presentes; a partir de uma matriz de adjacências podemos também atribuir um arco para todos os termos não nulos (estamos assumindo $M_{i \rightarrow i}=0$ sempre). Dessa forma, podemos traduzir um grafo de um tipo em um grafo de outro tipo, o que será visto com mais detalhe adiante. Em um grafo $G$, se existe uma aresta entre $i$ e $j,\{i, j\}$, diz-se que os nós $i$ e $j$ estão conectados e se existe um arco $(i, j)$, entre $i$ e $j$, diz-se que $i$ aponta para $j$ e que $j$ é apontado por $i$, o que será denotado por $i \rightarrow j$.

Podemos representar um grafo graficamente usando pontos (círculos ou outros símbolos) indexados para os sítios e segmentos (possivelmente curvos) sem orientação para as arestas. Para um grafo direcionado os arcos são representados por segmentos orientados, de forma que se $V_{1} \rightarrow V_{2}$ o segmento aponta de $V_{1}$ para $V_{2}$. Eventuais pesos que existam podem ser anexados a essas arestas (no caso direcionado iremos sempre anexar perto da cabeça da flecha). Essa representação gráfica implica que podemos pensar no grafo como sendo também um objeto abstrato, que codifica uma geometria (de fato uma métrica no sentido usual define um grafo ponderado). Um exemplo pode ser encontrado na figura 1.2.1. O grafo dessa figura será usado em vários dos exemplos adiante e será referido como $\mathcal{S}$ apenas.

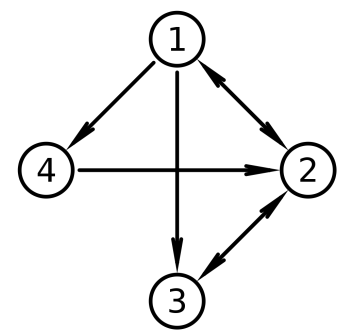

Figura 1.2.1. Representação de um grafo $(\mathcal{S})$.

Esqueletos direcionados e não-direcionados Seja $G$ um grafo com matriz de adjacências $M_{i \rightarrow j}$, vamos definir o seu esqueleto direcionado $\operatorname{Sk}_{d i r}(G)$ como sendo o grafo direcionado com matriz de adjacências:

$$
S_{i \rightarrow j}= \begin{cases}0, & \text { se } M_{i \rightarrow j}=0 \\ 1, & \text { caso contrário }\end{cases}
$$

e o seu esqueleto não-direcionado $\operatorname{Sk}(G)$ é definido como o grafo não-direcionado que tem matriz de adjacências

$$
S_{i \rightarrow j}= \begin{cases}0, & \text { se } M_{i \rightarrow j}=0 \text { e } M_{j \rightarrow i}=0 \\ 1, & \text { caso contrário }\end{cases}
$$

Um exemplo está na figura 1.2.2. 


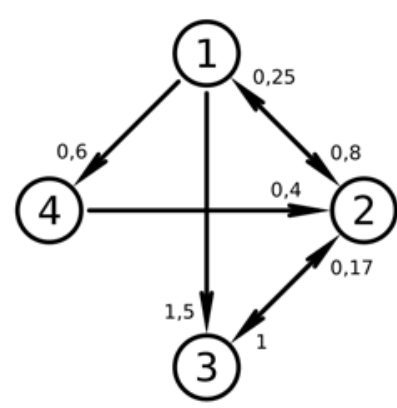

(a) $G$

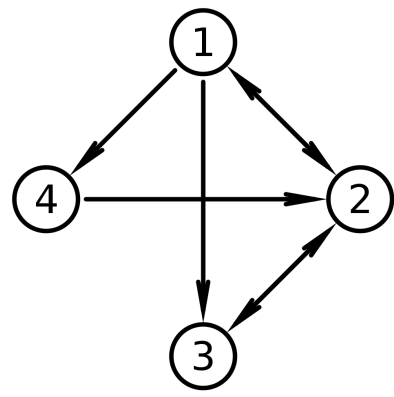

(b) $\operatorname{Sk}_{d i r}(G)$

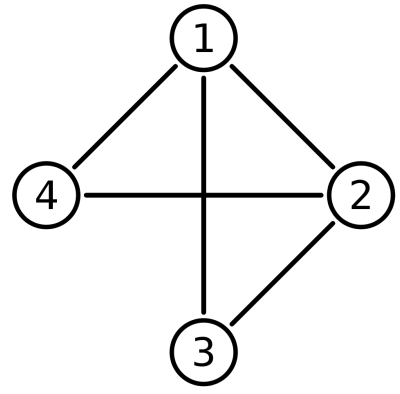

(c) $\operatorname{Sk}(G)=\operatorname{Sk}\left(\operatorname{Sk}_{d i r}(G)\right)$

Figura 1.2.2. Um grafo ponderado e os seus esqueletos, direcionado e não-direcionado.

Grafo reverso Dado um grafo direcionado (ou ponderado) $G$, podemos definir o seu reverso "invertendo a direção de todos os seus arcos". Em termos da matriz de adjacências isso significa que, se $G^{\prime}$ for o reverso de $G$, então $M\left(G^{\prime}\right)=M(G)^{T}$ (por essa razão ele também é conhecido como grafo transposto e denotado por $G^{T}$ em algumas referências). O reverso de $\mathcal{S}$ pode ser visto na figura 1.2.3.

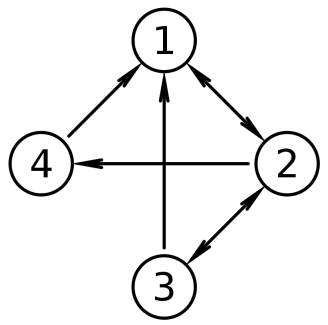

Figura 1.2.3. O grafo $\mathcal{S}^{T}$.

Complemento de um conjunto de nós Se $G$ for um grafo com nós $V(G)$ e $\Delta$ for um subconjunto de $V$, então o complemento de $\Delta$, denotado por $\bar{\Delta}$ é $V(G)-\Delta$, ou seja, os nós do grafo que não estão em $\Delta$. Por exemplo, $\overline{\{2,3\}}=\{1,4\} \mathrm{em} \mathcal{S}$.

Conjunto predecessor e sucessor, vizinhos e graus de um nó Dado um nó $i$ de um grafo, podemos definir o seu conjunto predecessor, denotado por $i_{-}$, como sendo o conjunto de todos os nós do grafo que apontam para $i$. De forma análoga, o sucessor é definido como o conjunto dos nós apontados por $i$ e denotado por $i_{+}$. Podemos ainda definir o conjunto de vizinhos de $i, \Gamma_{i}$, como sendo o conjunto de nós que fazem parte de alguma 
aresta do grafo junto com $i$ (o que significa também que $\Gamma_{i}=i_{-} \cup i_{+}$). Podemos usar esses conjuntos (mais precisamente os tamanhos deles) para definir a noção de grau de um sítio. O grau de entrada $q_{i}^{-}$é $\left|i_{-}\right|$, o grau de saída $q_{i}^{+}$é $\left|i_{+}\right|$e o grau de $i, q_{i}$ é o seu número de vizinhos, ou seja $\left|\Gamma_{i}\right|$. As definições de conjunto sucessor e predecessor podem ser extendidas para conjuntos de nós como sendo o conjunto de sítios que são respectivamente apontados por algum nó do conjunto ou que apontam para algum nó do conjunto, o que implica que

$$
\Delta_{-}=\bigcup_{i \in \Delta} i_{-} \quad \text { e } \quad \Delta_{+}=\bigcup_{i \in \Delta} i_{+} .
$$

Usando $\mathcal{S}$ como exemplo, $3_{-}=\{1,2\},\{1,4\}_{-}=\{1,2\},\{2,3\}_{+}=\{1,2,3\}$ e $\Gamma_{2}=\{1,3,4\}$.

Fontes e sorvedouros Uma fonte é um conjunto de nós aonde "não entram conexões" e um sorvedouro é um conjunto de nós de onde "não saem conexões". O que isso significa é que um sítio fora de uma fonte não aponta para nenhum nó dentro dela e um sítio fora de um sorvedouro não é apontado por nenhum elemento dentro dele. De forma equivalente, $\Delta$ é uma fonte sss $\Delta_{-} \subseteq \Delta$ e um sorvedouro sss $\Delta_{+} \subseteq \Delta$. Além disso, se $\Delta$ é uma fonte (sorvedouro) de um grafo ele é um sorvedouro (fonte) do grafo reverso. No grafo da figura 1.2.4 temos que $\{7\}$ é uma fonte e $\{4,5,6,7\}$ é um sorvedouro.

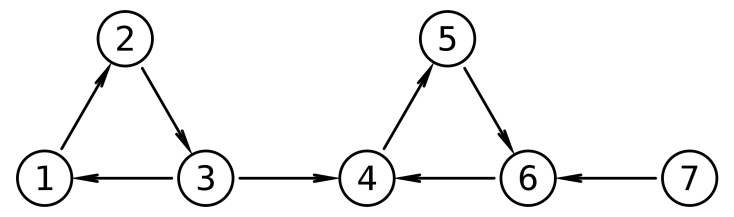

Figura 1.2.4.

Subgrafos e subgrafos induzidos Um subgrafo de $G$ é essencialmente um grafo $G^{\prime}$ construído a partir de alguns dos vértices de $G$ e tal que todas as relações definidas em $G^{\prime}$ já existiam em $G$. Ou seja, $V\left(G^{\prime}\right) \subseteq V(G), E\left(G^{\prime}\right) \subseteq E(G)$ e $A\left(G^{\prime}\right) \subseteq A(G)$. Para um grafo ponderado é comum se exigir que os pesos permaneçam os mesmos para os arcos e arestas que tenham sido "carregados" para o subgrafo. Dado um conjunto de nós não-vazio, $\Delta$, pode-se definir o subgrafo induzido por $\Delta$, denotado $G_{\Delta}$, como sendo o subgrafo tal que $V\left(G_{\Delta}\right)=\Delta$ e que todas as relações entre esses vértices que existiam em $G$ também existem em $G_{\Delta}$. Mais precisamente, $E\left(G_{\Delta}\right)=\{\{i, j\} \mid i, j \in$ $\Delta$ e $\{i, j\} \in E(G)\}$ e $A\left(G_{\Delta}\right)=\{(i, j) \mid i, j \in \Delta$ e $(i, j) \in A(G)\}$. Em termos da matriz de adjacências, $M\left(G_{\Delta}\right)$ é a submatriz principal de $M(G)$ obtida mantendo somente os índices que correspondem aos nós em $\Delta$. Finalmente dizemos que um 
subgrafo $G^{\prime}$ de $G$ é próprio se existe $\Delta \subsetneq V(G)$ tal que $G^{\prime}=G_{\Delta}$ (note que na literatura essa é a definição de um subgrafo próprio induzido. A definição usual de subgrafo próprio requer apenas que $G^{\prime} \neq G$ ). O subgrafo induzido em $\mathcal{S}$ pelo conjunto $\{2,3,4\}$, $\mathcal{S}_{\{2,3,4\}}$, pode ser encontrado na figura 1.2.5.

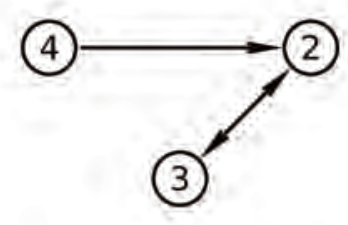

Figura 1.2.5. O subgrafo induzido em $\mathcal{S}$ pelo conjunto $\{2,3,4\}$.

Conjuntos e grafos minimais e maximais Dado um grafo $G$ e sendo $G^{\prime}$ um subgrafo induzido de $G$, diz-se que $G^{\prime}$ é minimal com respeito a uma propriedade $P$ se $G^{\prime}$ exibe essa propriedade e nenhum subgrafo próprio de $G^{\prime}$ exibe essa propriedade. De forma análoga, diz-se que $G^{\prime}$ é maximal se ele não é o subgrafo próprio de nenhum grafo induzido de $G$ obedecendo essa propriedade. De maneira equivalente, podemos falar de conjuntos de vértices maximais e minimais com respeito a uma certa propriedade (uma vez que só se está falando de grafos induzidos, o grafo define o conjunto de vértices e vice-versa. Por causa dessa conexão vamos exigir que tais conjuntos não sejam vazios). Por exemplo, uma fonte minimal é um conjunto de vértices $\Delta$ tal que $\Delta_{-} \subseteq \Delta$ e nenhum $\Delta^{\prime} \subsetneq \Delta$ é tal que $\Delta_{-}^{\prime} \subseteq \Delta^{\prime}$, a menos que $\Delta^{\prime}=\varnothing$ (nesse caso, a propriedade equivalente para grafos seria possuir um conjunto de vértices que fosse uma fonte de $G$ ). Na figura 1.2.4, $\{1,2,3,4,5,6\}$ é um sorvedouro, porém ele não é minimal pois existe o subconjunto próprio $\{4,5,6\}$ que também é um sorvedouro (esse sim é minimal).

Grafos completos Um grafo completo é um grafo não-direcionado que possui todas as arestas possíveis (ou seja, $E=\{\{i, j\} \mid i, j \in V$ e $i \neq j\}$ ). Um grafo direcionado completo é um grafo direcionado que possui todos os arcos possíveis $(A=\{(i, j) \mid i, j \in V$ e $i \neq$ $j\})$. Um grafo completo com 4 sítios pode ser encontrado na figura 1.2.6

Conjuntos independentes Um conjunto de sítios $\Delta$ é independente sss não existem conexões entre os vértices que o compõe. Note que se $\Delta$ é independente, segue que $\Delta_{-}, \Delta_{+} \subseteq \bar{\Delta}$. Um conceito importante é o de conjunto independente maximal, ou seja, um conjunto independente em que a adição de mais um vértice destrói a propriedade de independência. Como todo subconjunto próprio de um conjunto independente também é independente, segue que para caracterizar os conjuntos independentes de um grafo, basta encontrar os maximais. Em $\mathcal{S},\{1\}$ e $\{3,4\}$ são conjuntos independentes maximais, enquanto que $\{3\}$ é independente mas não maximal. 


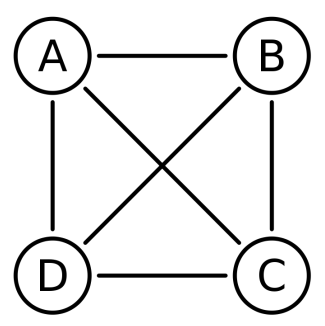

Figura 1.2.6. Um grafo completo com 4 sítios.

Cliques Um clique é um conjunto de vértices que induz um subgrafo completo, logo os seus elementos possuem todas as conexões possíveis entre si. No estudo de redes complexas eles estão ligados com a idéia de aglomeração e surgem por causa de efeitos do tipo $o$ amigo do meи amigo é meu amigo. No grafo da figura 1.2.7, o conjunto $\{A, B, C, D\}$ é um clique com 4 vértices.

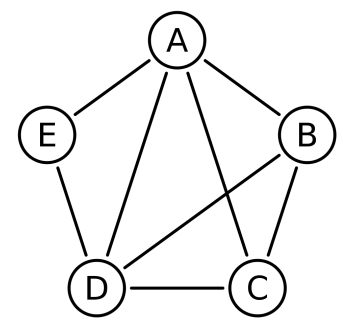

Figura 1.2.7. Um grafo com um clique de 4 sítios.

Uniões de grafos A união de 2 grafos $G$ e $H$, denotada $G \cup H$ é o grafo com todos os nós de $G$ e $H$, mas somente com as conexões que já existiam entre elementos de $G$ ou entre elementos de $H$ (em suma, isso significa que estamos nos referindo a 2 grafos sem relação entre si como partes de um mesmo grafo e sem fazer nenhuma mudança). O grafo $\mathcal{S}_{\{1,2,3\}} \cup \mathcal{S}_{\{4\}}$ se encontra na figura 1.2.8.

Caminhos, comprimentos e distâncias Definimos um caminho entre 2 sítios $v_{1}$ e $v_{2}$ como uma sequência de sítios conectados com o sítio anterior da sequência, que começa com $v_{1}$ e termina com $v_{2}$.Para um grafo direcionado isso significa que um sítio da sequência aponta para o seguinte $\left(v_{1} \rightarrow \ldots \rightarrow v_{2}\right)$. O comprimento de um caminho é o número de arestas ou arcos necessários para desenhar o caminho. Para um grafo ponderado o comprimento é definido as vezes como a soma dos pesos desses arcos. Finalmente, a distância entre 2 sítios é o menor comprimento possível para um caminho entre eles (ou $\infty$ se não existir nenhum caminho). Por exemplo, um sítio está a uma distância 


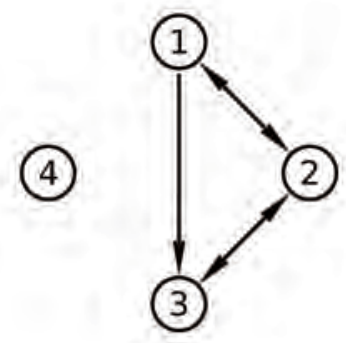

Figura 1.2.8. Exemplo de união de grafos. O grafo $\mathcal{S}_{\{1,2,3\}} \cup \mathcal{S}_{\{4\}}$.

0 dele mesmo e seus vizinhos estão a uma distância 1, no caso não-direcionado (note que só podemos garantir que a distância de $i$ a $j$ é igual a de $j$ até $i$ no caso nãodirecionado). Em $\mathcal{S}, 1 \rightarrow 4 \rightarrow 2$ é um caminho com comprimento 2. Além disso, a distância de 1 até 4 é 1 , mas a de 4 até 1 é 2 .

Componentes, grafos conexos e fortemente conexos Um grafo é conexo se ele não pode ser escrito como a união de 2 grafos e uma componente é um subgrafo induzido conexo maximal (ela não pode ser quebrada em 2 e não pode ser aumentada). Finalmente, um grafo é fortemente conexo se para todo $i$ e $j$ vértices desse grafo existe um caminho de $i$ a $j$ (note que em um grafo não-direcionado todo grafo conexo também é fortemente conexo). $\mathcal{S}, \mathcal{S}_{\{4\}}$ e $\mathcal{S}_{\{1,2\}}$ são fortemente conectados, mas $\mathcal{S}_{\{1,3\}}$ não é, porque não existe um caminho de 3 até 1 nele. $\mathcal{S}_{\{3,4\}}$ não é conexo pois ele pode ser escrito como $\mathcal{S}_{\{3\}} \cup \mathcal{S}_{\{4\}}$.

\subsubsection{Redes complexas e redes sociais}

Até a década de 90 a maior parte dos esforços para entender a teoria de grafos era focada em grafos com algum tipo de regularidade (como grafos completos, ciclos, reticulados, árvores ou grafos bipartites), em grafos aleatórios (seguindo o modelo de Erdös-Rényi), nos problemas de análise combinatória (como colorir grafos, calcular o polinômio de Tutte ou problemas da teoria de Ramsey), na existência de ciclos e recobrimentos (subgrafos ou conjuntos de subgrafos) com propriedades específicas (como a existência de ciclos hamiltonianos ou a conjectura do recobrimento duplo por ciclos), em problemas de otimização (como encontrar o maior subgrafo com uma propriedade dada, o teorema de fluxo máximo-corte mínimo ou o problema do caxeiro viajante) e na importância desse arcabouço teórico para a ciência da computação.

Desde então, com a disponibilidade de dados e o aumento da capacidade computacional, diversos cientistas começaram a aplicar essa teoria para caracterizar diversas redes observadas à nossa volta: a internet, a WWW, a rede que liga atores que já trabalharam juntos, a rede que liga cientistas que já colaboraram, redes de contatos sexuais de uma comunidade, redes 
que formam cadeias alimentares em ecossistemas, a rede de telefones que já fizeram uma ligação entre si, a rede de citações entre artigos científicos, redes relacionadas com aspectos linguísticos de um idioma e redes formadas por neurônios ${ }^{[5]}$. Ao contrário do que se suponha, redes complicadas como todas essas citadas não seguem as propriedades essenciais de grafos aleatórios previstas pelos trabalhos de Erdös e Rényi, embora apresentem inúmeras novas características comuns. O estudo dos processos que levam a criação de redes com as novas propriedades estatísticas observadas e de modelos que usem redes desse tipo para relacionar os seus agentes criou todo um novo ramo de pesquisa e a mecânica estatística se mostrou uma ferramenta valiosa nesses estudos. Iremos definir agora algumas quantidades que normalmente são usadas para caracterizar essas redes.

Diâmetro Definimos o diâmetro $d_{G}$ de um grafo como a distância máxima entre 2 sítios de G.

Distância média Definimos a distância média $\ell_{G}$ de um grafo $G$ como a média da distância entre todos os pares de sítios da rede:

$$
\ell_{G}=\frac{1}{N(N-1)} \sum_{v_{1}, v_{2} \in V(G)} d\left(v_{1}, v_{2}\right) .
$$

Aglomeração (ou clustering) Esta propriedade está associada à existência, no grafo, de cliques (no caso mais simples, de 3 sítios). As grandezas que medem o quanto uma rede possui cliques (tipicamente em um grafo não-direcionado) são chamados de coeficientes de aglomeração. O coeficiente de aglomeração pode ser definido de maneira local ou global. Se $E_{i}$ for o número de arestas entre os vizinhos de $i$ (de maneira mais rigorosa, o número de arestas do grafo induzido por $\Gamma_{i}$ ), temos que $i$ e seus vizinhos formam um clique se e somente se $E_{i}=\left(q_{i}\left(q_{i}-1\right)\right) / 2$. Definimos o coeficiente de aglomeração local como sendo então

$$
C_{i}= \begin{cases}\frac{2 E_{i}}{q_{i}\left(q_{i}-1\right)}, & \text { se } q_{i}>1 \\ 0, & \text { se } q_{i} \leq 1\end{cases}
$$

Podemos definir um coeficiente de aglomeração global como sendo a média dos $C_{i}$ ou, de maneira diferente, como sendo a razão entre 3 vezes o número de triângulos (cliques com 3 sítios) na rede e o número de triplas de sítios conectados (note que essa definição é focada em cliques de 3 sítios). As 2 definições não são equivalentes e, a menos que explicitamente indicado, estaremos nos referindo nesse texto à segunda.

Distribuição de graus Tipicamente sítios diferentes de uma rede vão ter graus (de entrada ou de saída) diferentes. Podemos então determinar, para um dado grafo, a distribuição de probabilidades que um sítio, escolhido ao acaso, tenha um certo grau de entrada ou de saída, ou ainda um certo número de vizinhos. Uma vez determinada a distribuição (chamada distribuição de graus $p(k)$ ), podemos analisar suas propriedades estatísticas, como o grau médio. 
Assortatividade A assortatividade de uma rede mede a tendência dos sítios de conectividade alta (grau alto) de conectarem entre si. Uma rede com assortatividade alta em geral é formada por vários subgrafos altamente conectados e que possuem umas poucas conexões entre si. Se a rede tem assortatividade negativa, sítios de conectividade alta tendem a se conectar com sítios de conectividade baixa, e a rede é dita dissortativa. O coeficiente de assortatividade $r$ é definido como o coeficiente de correlação de Pearson para os graus dos sítios. Se $p(k)$ e $p(q, k)$ forem, respectivamente, a distribuição de graus (probabilidade de um sítio qualquer possuir $k$ vizinhos) e a distribuição de graus entre vizinhos (probabilidade que um sítio qualquer e um vizinho qualquer dele tenham respectivamente $q$ e $k$ vizinhos), então

$$
r=\frac{1}{\sigma^{2}} \sum_{q, k} q k(p(q, k)-p(q) p(k))
$$

onde $\sigma$ é o desvio padrão da distribuição $p(k)$.

As propriedades mais marcantes que são compartilhadas por essas redes - que receberam genericamente o nome de redes complexas -, são as de mundo pequeno, livre de escala $\mathrm{e}$ aglomeração alta.

Mundo Pequeno A propriedade de mundo pequeno identifica o fato de que, apesar dessas redes terem tamanhos tipicamente grandes, todos os sítios estão relativamente próximos uns dos outros, ou seja, podemos partir de qualquer ponto e chegar em qualquer outro através de um caminho relativamente curto.

Devemos salientar que essa propriedade não é exclusiva das redes complexas, uma vez que os grafos aleatórios do modelo de Erdös-Rényi são tais que $\ell \propto \ln (N)$. Porém reticulados (como redes quadradas e redes cúbicas) não exibem essa propriedade.

Aglomeração alta Um fato que diferencia uma rede complexa de um grafo aleatório com o mesmo número de sítios e arestas é que o coeficiente de aglomeração é sistematicamente maior nas redes complexas. Em redes sociais, isso se deve a fenômenos do tipo $O$ amigo do meu amigo também é meu amigo.

Livre de escala A distribuição de graus para um grafo aleatório segue uma distribuição de Poisson, enquanto que tipicamente uma rede complexa tem uma distribuição de graus dada por uma lei de potências. As redes que possuem distribuições desse último tipo são chamadas livres de escala e possuem estruturas com auto-similaridade. Isso revela o fato de que essas redes são mais parecidas com redes fractais (como o grafo de Cayley, a rede de Bethe e a rede de Apolônio) do que com grafos aleatórios. No entanto, nem sempre uma rede livre de escala tem uma estrutura hierárquica clara. 
A constatação de que muitas redes reais apresentavam essas propriedades motivou uma investigação acerca de processos e mecanismos que, presentes durante a formação da rede, pudessem lhes dar origem. Dois processos em particular geram modelos de crescimento de redes que são bastante empregados, o redirecionamento aleatório de arestas, capaz de gerar uma rede com propriedades de mundo pequeno a patir de uma rede inicial em que essa propriedade não esteja presente, também conhecido como modelo de Watts-Strogatz (WS) ${ }^{[4]}$ e o processo de conexões preferenciais, capaz de gerar uma rede livre de escala, e que é conhecido como modelo de Barabási-Albert (BA) ${ }^{[5]}$.

\section{Rede de Watts-Strogatz}

O modelo de Watts-Strogatz original ${ }^{[47]}$ é um modelo de 2 parâmetros, que é em um certo sentido uma interpolação entre um grafo regular e um grafo aleatório. Começamos com um grafo sem arestas e numeramos os sítios de 1 a $N$; em seguida, conectamos o sítio $i$ com os sítios $i \pm 1, i \pm 2, \ldots, i \pm K$ (módulo $N$ ), onde $K$ é um parâmetro do modelo que deve ser um inteiro positivo tal que $N \gg K \gg \ln (N) \gg 1{ }^{1}$. Note que esse grafo inicial tem coeficiente de aglomeração alto, $C=\frac{3(K-1)}{2(2 K-1)}$, que tende para 0,75 quando $K$ se torna grande. $\mathrm{O}$ passo seguinte é mudar cada aresta de maneira aleatória com probabilidade $p$ (o outro parâmetro do modelo). A idéia é que para $p=0$ temos uma rede regular e para $p=1$ temos um grafo aleatório. A rede regular tem a propriedade de alta aglomeração, mas a distância média também é alta; por outro lado, o grafo aleatório tem aglomeração e distância média baixas. O que torna o modelo interessante é que, analisando o coeficiente de aglomeração $C(p)$ e a distância média $\ell(p)$ em função do parâmetro $p$ (já no limite discutido de $K$ grande) e olhando as frações $C(p) / C(0)$ e $\ell(p) / \ell(0)$, vemos que a distância média diminui significativamente para valores de $p$ bem mais baixos do que os necessários para diminuir a aglomeração. Logo, existe uma janela de valores de $p$ em que a rede tem, ao mesmo tempo, a propriedade de mundo pequeno e aglomeração alta.

A distância média $\ell$ depende de $p, N$ e $K$ segundo

$$
\ell \simeq \frac{N}{K} f(p K N) \quad \text { com } \quad f(u)=\frac{4}{\sqrt{u^{2}+4 u}} \operatorname{arctgh}\left(\frac{u}{\sqrt{u^{2}+4 u}}\right)
$$

onde o regime em que $f$ aproxima bem a forma de $\ell$ é $u \ll 1$ ou $u \gg 1$, de forma que $\ell(p) / \ell(0)=f(p K N)$ e o regime de mundo pequeno é dado por $p K N \gg 1$. Nesse caso, a distância média cresce logaritmicamente com o tamanho da rede e obtemos a seguinte forma assintótica para $\ell$,

$$
\ell(p, K, N) \simeq \frac{\ln (p K N)}{p K^{2}}
$$

\footnotetext{
${ }^{1}$ Essa condição é importante para que tenhamos inicialmente uma rede que não seja de mundo pequeno, mas que adquira propriedades interessantes após o próximo passo
} 
O coeficiente de aglomeração pode ser estimado para valores pequenos de $p$ levando em conta que, se um triângulo existia na rede regular, ele não é destruído pelas reconexões com probabilidade $(1-p)^{3}$. De forma que $C(p) / C(0) \simeq(1-p)^{3}$ e essa aproximação deve melhorar a medida que aumentamos $N$. Segue que, se fixarmos um valor pequeno mas não nulo de $p$ e aumentarmos o suficiente o tamanho da rede, sempre teremos aglomeração alta e propriedades de mundo pequeno. Por outro lado, nem a distribuição de graus da rede regular, nem a da rede aleatória são livres de escala. A distribuição de graus da rede de Watts-Strogatz acaba sendo uma interpolação entre uma função delta e uma distribuição de Poisson, com um pico bem determinado e decaimento exponencial.

\section{Rede de Barabási-Albert}

O modelo Barabási-Albert ${ }^{[4]}$ (que por sua vez é baseado em um modelo mais antigo devido a Price ${ }^{[31]}$ ) tem apenas um parâmetro $m$ que dita como ocorre o crescimento da rede a partir de uns poucos sítios iniciais. Começamos com um grafo completo com $m+1$ sítios; a cada sítio que acrescentamos escolhemos $m$ sítios distintos, que já estão na rede. A idéia das conexões preferenciais é que a probabilidade do sítio novo se conectar com um sítio $i$ da rede é proporcional a $q_{i}$, ou seja, ao número de conexões que o sítio $i$ já possui.

A rede que resulta é livre de escala com uma distribuição de graus $p(q) \simeq q^{-3}$ e uma distância média $\ell \simeq \ln (N) / \ln (\ln (N))$. O coeficiente de aglomeração, por outro lado, segue uma lei de potência, $C \simeq N^{-0,75}$ e, ao contrário da rede de Watts-Strogatz, diminui com o tamanho da rede. Por outro lado, se olharmos para uma rede aleatória com o mesmo número de sítios e mesmo grau médio veremos que o seu coeficiente de aglomeração é $C \simeq 1 / N$, portanto menor ainda.

Podemos discutir a importância das conexões preferenciais olhando o que acontece quando temos versões não lineares dessa regra e $m=1$, nas quais a probabilidade de que o sítio novo se conecte com $i$ é proporcional a $q_{i}^{\alpha}$. O caso $\alpha>1$ cria hubs que tem quase todas as conexões. Mais precisamente, se

$$
k(\alpha)=\left\lceil\frac{1}{\alpha-1}\right\rceil
$$

então o número de sítios com grau maior que $k(\alpha)$ é finito mesmo no limite em que o tamanho da rede é infinito. Se $q \leq k(\alpha)$, então o número de sítios com grau $q$ é proporcional a $N^{q-(q-1) \alpha}$, o que dá uma distribuição com decaimento exponencial e um corte em $q=k(\alpha)$. Para $\alpha<1$ é possível obter o resultado analítico

$$
p(q)=\frac{\mu}{q^{\alpha}} \prod_{j=1}^{q}\left(1+\frac{\mu}{j^{\alpha}}\right)^{-1},
$$

o que também dá uma distribuição exponencial ( $\mu$ é um parâmetro entre 1 e 2 que depende de $\alpha$ ). 
Finalmente, apesar da distribuição de graus não possuir desvio padrão no limite $N \rightarrow \infty$, podemos encontrar a forma assintótica da assortatividade da rede Barabási-Albert nesse mesmo limite, olhando redes finitas (isso pode ser feito introduzindo um cut-off na distribuição, uma vez que em uma rede de $N$ sítios não existem sítios com mais do que $N$ vizinhos). É possível mostrar que $r \rightarrow 0$, com $\log (N)^{2} / N$, de forma que não há preferência dos hubs nem de se conectarem entre si, nem de se "afastarem", ou seja, a rede não é nem assortativa, nem dissortativa.

\subsection{Conceitos de sistemas dinâmicos}

Ao longo de todo o texto, iremos usar conceitos de sistemas dinâmicos. A maior parte desses conceitos pode ser encontrada em diversos livros introdutórios sobre o assunto, como por exemplo ${ }^{[12]}$. Porém alguns dos conceitos são um pouco mais sofisticados, ou fogem do escopo de obras introdutórias, de forma que uma pequena introdução é necessária.

Iremos tratar apenas de sistemas autônomos, contínuos no tempo e definidos em $\mathbb{R}^{M}$, que são comumente chamados de fluxos na literatura. Mais precisamente, estamos nos referindo a equações do tipo

$$
\frac{\mathrm{d} \vec{x}}{\mathrm{dt}}=\vec{f}(\vec{x}),
$$

com $f: \mathbb{R}^{M} \rightarrow \mathbb{R}^{M}$. Iremos chamar de pontos fixos, os pontos onde a derivada no tempo de $\vec{x}$ se anula, ou seja os pontos $\vec{x}^{*}$ tais que

$$
\vec{f}\left(\vec{x}^{*}\right)=\overrightarrow{0} .
$$

Iremos chamar de trajetória o resultado da integração de um fluxo no tempo, partindo de alguma condição inicial.

Estaremos sempre interessados em resolver o comportamento qualitativo de um fluxo. Logo, ao invés de determinar exatamente quais são as trajetórias, iremos estabelecer como elas se parecem de um ponto de vista topológico (ou seja, permitindo deformações). Iremos sempre trabalhar em situações em que as coordenadas de $\vec{f}$ são infinitamente diferenciáveis e com a primeira derivada limitada no espaço de fases (a região do espaço em que estamos considerando a equação). Podemos garantir existência e unicidade das trajetórias pelo teorema de Picard-Lindelöf (a limitação nas primeiras derivadas implica continuidade Lipschitz) e a unicidade das trajetórias implica que trajetórias diferentes só podem se encontrar em pontos fixos. Além disso, a limitação nas derivadas também implica que pontos fixos demoram um tempo infinito para serem atingidos.

A informação qualitativa, sobre as trajetórias, que é mais simples de ser obtida é o seu comportamentos assintótico na vizinhança dos pontos fixos. Considere um ponto fixo $\vec{x}^{*}$. Podemos então expandir os primeiros termos da série de Taylor de $\vec{f}\left(\vec{x}^{*}+\vec{\epsilon}\right)$ em torno de 
$\vec{x}^{*}$ (usando a notação de Einstein, ou seja se um índice aparece repetido, subentende-se uma soma sobre esse índice e denotando $\partial^{k} / \partial x_{c_{1}}, \ldots, \partial x_{c_{k}}$ por $\left.\partial_{c_{1}, \ldots, c_{k}}^{k}\right)$ :

$$
\vec{f}\left(\vec{x}^{*}+\vec{\epsilon}\right)-\vec{f}\left(\vec{x}^{*}\right)=\frac{\epsilon_{i}}{1 !} \partial_{i} \vec{f}+\frac{\epsilon_{i} \epsilon_{j}}{2 !} \partial_{i, j}^{2} \vec{f}+\frac{\epsilon_{i} \epsilon_{j} \epsilon_{k}}{3 !} \partial_{i, j, k}^{3} \vec{f}+\ldots,
$$

onde todas as derivadas de $\vec{f}$ são avaliadas no ponto fixo $\vec{x}^{*}$. Intuitivamente, para $\vec{\epsilon}$ pequeno, só precisamos considerar o primeiro termo dessa expansão, ou seja, se $\mathcal{J}$ for o jacobiano de $\vec{f}$ avaliado no ponto fixo, a expansão seria truncada como $\mathcal{J} \vec{\epsilon}$. O teorema de HartmanGrobman nos diz que, se esse jacobiano tiver todos os autovalores com parte real não-nula, então o comportamento assintótico é regido apenas por esse termo, ou seja, precisariamos considerar a equação linear

$$
\frac{\mathrm{d} \vec{\epsilon}}{\mathrm{dt}}=\mathcal{J} \vec{\epsilon}
$$

de forma que o comportamento é todo determinado pelos autovalores do jacobiano. Os pontos fixos em que isso acontece são ditos hiperbólicos. Os casos em que os autovalores tem parte real nula precisam ser analisados levando em conta mais termos da expansão.

Em alguns casos teremos alguma grandeza conservada pelas equações do fluxo e caso isso ocorra podemos ter autovalores nulos. Porém, nesses casos específicos, os termos de ordem superior não precisam ser levados em conta na análise. Para entender o que queremos dizer com isso, suponha que uma função $\phi(\vec{x})$ (suficientemente diferenciável) obedece

$$
\frac{\mathrm{d} \phi}{\mathrm{dt}}=0
$$

e que estamos interessados em trajetórias tais que $\phi=a$. Essa equação de fato reduz o número de dimensões do espaço de fase em que as trajetórias que queremos estudar estão e poderiamos impor esse vínculo com uma mudança de coordenadas. Por outro lado, essa mudança de coordenadas pode complicar consideravelmente as equações e obscurecer a análise. Chamamos a atenção então para o fato que a equação 1.3.4 é equivalente a

$$
\vec{f} \cdot \nabla \phi=0
$$

e que tirando o gradiente em ambos os lados temos

$$
\vec{f} \cdot \mathcal{J}_{\nabla \phi}+\nabla \phi \cdot \mathcal{J}_{\vec{f}}=\vec{f} \cdot \mathcal{H}_{\phi}+\nabla \phi \cdot \mathcal{J}_{\vec{f}}=\overrightarrow{0}
$$

onde $\mathcal{H}$ denota a matriz hessiana. Logo em um ponto fixo temos

$$
\nabla \phi \cdot \mathcal{J}=\overrightarrow{0}
$$

e temos 2 situações possíveis, $\nabla \phi=\overrightarrow{0}$, o que implica que o ponto fixo é um ponto crítico de $\phi$ (isso é comum quando o ponto fixo é um centro, por exemplo), ou então $\nabla \phi$ é um 
autovetor à esquerda de $\mathcal{J}$ com autovalor $0^{2}$. Nesse segundo caso, o autovalor não tem relevância nenhuma para as trajetórias tais que $\phi=a$. Para ver isso, primeiro notamos que todos os autovetores à direita de $\mathcal{J}$ que não tem autovalor 0 devem ser perpendiculares a $\nabla \phi$. Considere então o seguinte fluxo:

$$
\frac{\mathrm{d} \vec{x}}{\mathrm{dt}}=\vec{g}(\vec{x})=\vec{f}(\vec{x})+\mu \cdot(\phi(\vec{x})-a) \cdot \nabla \phi(\vec{x}) .
$$

As equações 1.3 .8 e 1.3.1 coincidem para $\phi=a$ e logo as trajetórias dentro dessa variedade devem ter as mesmas propriedades. Considerando o jacobiano de $\vec{g}(\vec{x})$ em um ponto fixo que obedeça $\phi=a$ temos

$$
\mathcal{J}+\mu . \nabla \phi \otimes \nabla \phi
$$

Logo, $\nabla \phi$ continua sendo um autovetor à esquerda, mas agora com autovalor $\mu \cdot|\nabla \phi|^{2}$ :

$$
\nabla \phi \cdot(\mathcal{J}+\mu \cdot \nabla \phi \otimes \nabla \phi)=\nabla \phi \cdot \mathcal{J}+\mu \cdot(\nabla \phi \cdot \nabla \phi) \cdot \nabla \phi=\mu \cdot|\nabla \phi|^{2} \cdot \nabla \phi
$$

e todos os autovetores $\vec{v}$ à direita, tais que $\nabla \phi \cdot \vec{v}=0$, continuam com o mesmo autovalor $\lambda$ :

$$
(\mathcal{J}+\mu \cdot \nabla \phi \otimes \nabla \phi) \cdot \vec{v}=\mathcal{J} \cdot \vec{v}+\mu \nabla \phi \cdot(\nabla \phi \cdot \vec{v})=\lambda \vec{v} .
$$

Usando os teoremas A.2.6 e A.2.8, vemos que todos os autovetores à direita, $\vec{v}$, que tem autovalor não-nulo e alguns dos que tem autovalor nulo, são tais que $\nabla \phi \cdot \vec{v}=0$, indicando que a direção $\nabla \phi$ não é relevante para a análise de estabilidade dentro da variedade $\phi=a$. Iremos nos referir ao longo do texto a autovalores e autovetores desse tipo como artefatos de imersão (Um caso com que iremos nos deparar é quando $\phi$ é a soma de todas as probabilidades, que permanece igual a 1 ao longo da evolução temporal).

Esse argumento pode ser facilmente generalizado para quando temos mais de uma grandeza conservada, desde que os gradientes dessas grandezas formem uma base linearmente independente em torno de uma vizinhança do ponto fixo que estamos considerando (isso pode ser visto como uma generalização da imposição que $\nabla \phi \neq \overrightarrow{0}$ ). Nesse caso, se os valores das grandezas conservadas $\phi_{i}$ forem $a_{i}$, consideramos para essa vizinhança o fluxo:

$$
\frac{\mathrm{d} \vec{x}}{\mathrm{dt}}=\vec{f}(\vec{x})+\sum_{i}\left(\phi_{i}(\vec{x})-a_{i}\right) \sum_{j} \alpha_{i, j}(\vec{x}) \cdot \nabla \phi_{j}(\vec{x}),
$$

cujo jacobiano no ponto fixo fica

\footnotetext{
${ }^{2}$ Um autovetor à direita, com autovalor $\lambda$, de uma matriz $A$ é um vetor $\vec{x} \neq \overrightarrow{0}$ (ou, uma matriz coluna), tal que $A \cdot \vec{x}=\lambda \vec{x}$. De forma análoga, um autovetor à esquerda, com autovalor $\lambda$ é um vetor $\vec{y} \neq \overrightarrow{0}$ (ou, uma matriz linha), tal que $\vec{y} \cdot A=\lambda \vec{y}$. Os autovetores à esquerda são essencialmente os autovetores à direita da matriz transposta e vice-versa, de forma que eles tem os mesmos autovalores. Algumas propriedades importantes de como esses dois tipos de autovetores se relacionam são elaboradas no apêndice A.2.
} 


$$
\mathcal{J}+\sum_{i, j} \alpha_{i, j} \cdot \nabla \phi_{j} \otimes \nabla \phi_{i}
$$

Os autovalores não-nulos são trivialmente preservados (pela mesma razão que no caso com uma grandeza conservada). Multiplicando à esquerda por $\nabla \phi_{i}$ vem

$$
\sum_{j, k} \alpha_{j, k} \cdot \nabla \phi_{i} \cdot\left(\nabla \phi_{k} \otimes \nabla \phi_{j}\right)=\sum_{j, k} \alpha_{j, k} \cdot\left(\nabla \phi_{i} \cdot \nabla \phi_{k}\right) \cdot \nabla \phi_{j},
$$

que queremos igualar a $\lambda_{i} \cdot \nabla \phi_{i}$, através de uma escolha dos $\alpha$ (que são funções que ainda não especificamos). Definindo as matrizes $A=\left(\alpha_{i, j}\right), \Phi=\left(\nabla \phi_{i} . \nabla \phi_{j}\right)$ e $\Lambda=\left(\lambda_{i} \delta_{i, j}\right)$, segue da equação 1.3.14 que $\nabla \phi_{i}$ tem autovalor $\lambda_{i}$ para todo $i$ sss

$$
A . \Phi=\Lambda \Rightarrow A=\Lambda . \Phi^{-1},
$$

lembrando que o fato de $\left\{\nabla \phi_{i}\right\}$ ser uma base linearmente independente implica que $\Phi$ é inversível. Usando essa escolha para a matriz $A$, podemos modificar os autovalores provenientes dos artefatos da maneira que quisermos.

Outros dois conceitos relacionados a pontos fixos que serão usados são o conceito de isolamento e índice. Um ponto fixo é dito isolado, se existe uma vizinhança aberta dele em que ele é o único ponto fixo. Pelo teorema da função implícita, para que um ponto fixo seja isolado, basta que $\mathcal{J}$ seja inversível. Como o determinante de uma matriz é o produto de seus autovalores, isso é o mesmo que dizer que o 0 não é um autovalor. Note que artefatos de imersão não precisam ser levados em conta, dependendo das informações em que estivermos interessados. Por exemplo, se $\phi$ for conservada pela evolução temporal e estivermos interessados na variedade $\phi=a$, então para checar se um ponto fixo tal que $\nabla \phi \neq$ $\overrightarrow{0}$ é isolado com respeito a topologia natural da variedade $\phi=a$ (a topologia induzida por essa variedade, assumindo que estamos usando a topologia euclideana para $\mathbb{R}^{M}$ ), podemos ignorar o autovalor correspondendo ao autovetor à esquerda, $\nabla \phi$ (pelo mesmo argumento que usamos, envolvendo o fluxo 1.3.8). A definição de índice que iremos utilizar é uma simplificação para pontos fixos em que $\mathcal{J}$ é inversível. Nesse caso, o índice de um ponto fixo é:

$$
\left\{\begin{aligned}
-1, & \text { se } \operatorname{det}(\mathcal{J})<0 \\
1, & \text { se } \operatorname{det}(\mathcal{J})>0
\end{aligned}\right.
$$

Como $\mathcal{J}$ é uma matriz real, isso é o mesmo que dizer que o índice é $(-1)^{s}$, onde $s$ é o número de autovalores com parte real negativa. Novamente, se tivermos artefatos, eles podem ser ignorados e podemos usar $(-1)^{s}$ como o índice para o ponto fixo na variedade correspondente, por exemplo, $\phi=a$.

Finalmente iremos usar o conceito de bifurcação e de estabilidade estrutural. Essencialmente, uma bifurcação local acontece quando temos um fluxo que depende de alguns parâmetros, por exemplo 


$$
\frac{\mathrm{d} \vec{x}}{\mathrm{dt}}=\vec{f}(\vec{p} ; \vec{x}),
$$

onde $\vec{p}$ são os parâmetros fixos e uma modificação desses parâmetros modifica qualitativamente a estrutura dos pontos fixos. Para uma bifurcação global, a perturbação muda topologicamente quais são as trajetórias. Vamos dizer que uma bifurcação ocorre para um certo conjunto de parâmetros se existem perturbações arbitrariamente pequenas deles que causem uma bifurcação. Estabilidade estrutural é um conceito relacionado, mas que vai além de perturbações dos parâmetros e da estrutura de pontos fixos. A idéia é que um fluxo é estruturalmente estável se qualquer perturbação $\vec{g}(\vec{x})$, contínua, com a primeira derivada contínua e suficientemente pequena (em algum sentido) é tal que as trajetórias são topologicamente iguais a do fluxo original (ou seja, o fluxo é qualitativamente o mesmo). É possível fazer uma conexão entre os 2 conceitos reescrevendo essa perturbação de forma que o fluxo seja

$$
\frac{\mathrm{d} \vec{x}}{\mathrm{dt}}=\vec{f}(\vec{p} ; \vec{x})+\mu \vec{g}(\vec{x})
$$

e consideramos $\mu=0$ para o fluxo original. Uma ferramenta poderosa para o estudo de bifurcações locais é o teorema da função implícita, já que ele implica que perturbações nos parâmetros ou no fluxo não alteram os pontos fixos hiperbólicos, de forma que para buscar bifurcações locais é necessário procurar condições de existência de pontos não hiperbólicos. Por outro lado é possível criar bifurcações globais sem bifurcações locais, como o surgimento das janelas de periodicidade no sistema de Lorenz ${ }^{[29]}$, de forma que uma análise dos pontos fixos não é suficiente para encontrar essas bifurcações. 


\section{Capítulo 2}

\section{Definições dos modelos}

“All models are wrong, but some are useful."

- George E. P. Box

"The purpose of models is not to fit the data but to sharpen the questions."

- Samuel Karlin

Nessa seção iremos apresentar alguns dos modelos que são usados no estudo de propagação de opiniões e alguns resultados conhecidos deles, assim como algumas idéias que são comuns a vários modelos.

\subsection{Modelagem com redes complexas e regras de confiança}

Em todos os modelos que iremos estudar, a sociedade é definida usando uma rede complexa, em que cada nó representa uma pessoa e cada aresta representa uma conexão social. Nós não iremos considerar efeitos como a intensidade dessas conexões sociais e para cada nó $i$, iremos representar o estado (interpretado como uma opinião) da pessoa correspondente por $\sigma_{i}$. Esse estado pode ser ou um número real, um número inteiro, ou mesmo um vetor de inteiros, dependendo do modelo.

Uma propriedade comum a vários modelos (apesar de ter realizações consideravelmente diferentes) é a confiança limitada (bounded confidence), que essencialmente diz que 2 pessoas com opiniões diferentes só interagem se elas tem opiniões que não são tão diferentes. Mais precisamente, quanto maior $\left\|\sigma_{i}-\sigma_{j}\right\|$ para uma certa norma, menor a probabilidade da interação (normalmente com um cut-off a partir do qual não há interação). Nos modelos com opiniões discretas (números inteiros, principalmente) é comum usar uma confiança limitada em que $i$ e $j$ interagem se e somente se $\left|\sigma_{i}-\sigma_{j}\right| \leq 1$, ou seja, se as suas opiniões forem consecutivas. 


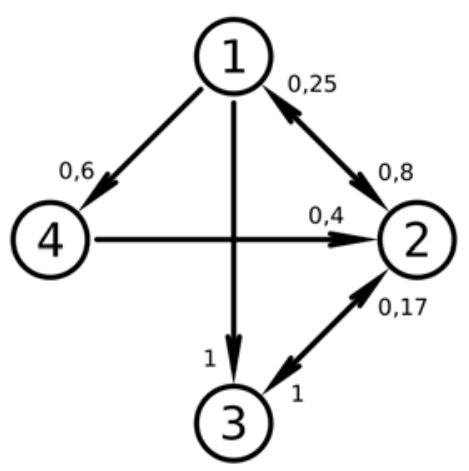

Figura 2.1.1. Representação da regra de confiança, em que temos 4 opiniões, com $p_{1 \rightarrow 2}=0,8$; $p_{1 \rightarrow 3}=1 ; p_{1 \rightarrow 4}=0,6 ; p_{2 \rightarrow 1}=0,25 ; p_{2 \rightarrow 3}=1 ; p_{3 \rightarrow 2}=0,17 ; p_{4 \rightarrow 2}=0,4$ e $p_{\sigma \rightarrow \sigma^{\prime}}=0$ nos outros casos.

Uma das principais contribuições desse trabalho é a generalização das regras de confiança limitada, para o que chamamos de regras de confiança generalizadas. Em $^{[43]}$, nós propusemos esse conceito que engloba a ideia de confiança limitada e que pode ser encarado como uma modelagem de viéses e preconceitos, podendo ser aplicada a qualquer modelo com opiniões discretas. Nela, nós definimos a priori um conjunto de parâmetros $\left\{p_{\sigma \rightarrow \sigma^{\prime}}\right\}$, onde $p_{\sigma \rightarrow \sigma^{\prime}}$ significa a probabilidade que uma interação que mude a opinião de um agente de $\sigma$ para $\sigma^{\prime}$ de fato ocorra. Por exemplo, se as regras usuais de um modelo dizem que um sítio com opinião 3 deve passar a ter opinião 1, então usando essa modificação, com probabilidade $p_{3 \rightarrow 1}$ a mudança de 3 para 1 de fato ocorre, do contrário, o sistema permanece no mesmo estado. Note também que não importa qual seja a regra, os parâmetros $p_{\sigma \rightarrow \sigma}$ são irrelevantes para todos os valores possíveis de opinião $\sigma$ (mudar o valor de uma opinião para o valor dela antes da interação é o mesmo que a interação não ser bem sucedida, já que os estados finais da rede são os mesmos), de forma que vamos sempre tomar esses parâmetros como sendo 0 .

Uma representação esquemática que será bastante útil é obtida interpretando esses parâmetros $p_{\sigma \rightarrow \sigma^{\prime}}$ como sendo os pesos de um grafo ponderado (nós iremos chamar de regra de confiança tanto um conjunto de parâmetros quanto o grafo associado a ele), o que permite uma representação gráfica (ver figura 2.1.1).

É importante sublinhar que podemos usar regras de confiança para recuperar não apenas a confiança limitada, mas também algumas outras modificações que são comuns:

- Se $p_{\sigma \rightarrow \sigma^{\prime}}=1$ para todo $\sigma \neq \sigma^{\prime}$, então temos um modelo sem confiança limitada. Isso é o que acontece, por exemplo, na versão usual do modelo Sznajd e do modelo do votante, que serão os modelos mais estudados nos próximos capítulos.

- Se $p_{\sigma \rightarrow \sigma^{\prime}}$ depende apenas de $\left\|\sigma-\sigma^{\prime}\right\|$ recupera-se a regra de confiança limitada. Em particular, a regra que vamos usar no modelo Sznajd é $p_{\sigma \rightarrow \sigma^{\prime}}=1$ se $\left|\sigma-\sigma^{\prime}\right|=1 \mathrm{e}$ 
$p_{\sigma \rightarrow \sigma^{\prime}}=0$ caso contrário, que é a regra de confiança limitada mais usual.

- Se $\sigma$ é uma opinião tal que para todo $\sigma^{\prime} \neq \sigma$ temos $p_{\sigma \rightarrow \sigma^{\prime}}=p$ e $p_{\sigma^{\prime} \rightarrow \sigma}=0$, então $\sigma$ pode ser interpretado como um estado de indecisão.

- Regras de confiança permitem interações cíclicas do tipo pedra $(R)$, papel $(P)$, tesoura $(S)$, em que temos $p_{R \rightarrow P}=p_{P \rightarrow S}=p_{S \rightarrow R}=p$ e $p_{P \rightarrow R}=p_{S \rightarrow P}=p_{R \rightarrow S}=q$, com $p>q$.

\subsection{Convencimento, Adaptação e Homogeneização}

De forma geral, as regras de interação entre os agentes dos modelos de propagação de opinião costumam ser de três tipos:

Convencimento Nesse tipo de interação, um grupo de sítios com uma certa opinião é escolhido de alguma forma e um outro grupo de sítios, com opiniões que podem divergir das do primeiro grupo, também é escolhido. A seguir, o primeiro grupo tenta convencer os agentes do segundo grupo de sua opinião.

Adaptação Novamente, dois grupos de sítios são escolhidos segundo algum critério. Porém agora, um dos grupos (que não precisa necessariamente ter só uma opinião) observa as opiniões dos agentes do segundo grupo. Depois, seus agentes podem ou não mudar as suas opiniões, com base nessa nova informação.

Homogenização Nesse tipo de interação, um grupo de sítios é escolhido e simulamos um processo de discussão entre eles. Depois desse processo o grupo chega a um consenso, que não precisa ser uma opinião que algum dos agentes já tivesse, podendo ser um compromisso entre as diversas opiniões prévias dos agentes.

Note que a única interação que é simétrica entre os agentes que participam dela é a homogenização. Nos outros dois tipos de interação temos basicamente agentes tentando modificar o ambiente em que eles se encontram (convencimento) ou agentes se adaptando ao ambiente (adaptação). Essas duas interações também são conhecidas como de dentro para fora (outward) e de fora para dentro (inward). Uma questão que surgiu e que tem sido debatida na literatura ${ }^{[17 ; 27 ; 37]}$ é se existe alguma diferença essencial no resultado final da evolução de um modelo devida exclusivamente a esses dois tipos de dinâmica, um ponto que ainda não está completamente claro.

\subsection{O Modelo do Votante e o modelo Ochrombel}

O modelo do votante é um modelo de propagação de opiniões, baseado na idéia de processo de contato. A primeira versão deste modelo foi proposta em ${ }^{[22]}$ por Ligget. No modelo 
do votante a opinião de cada agente é representada por um número inteiro entre 1 e $M$. As regras do modelo, que definem como as opiniões evoluem, são:

- Escolhemos um sítio $i$ de maneira aleatória e um vizinho $j$ desse sítio.

- O sítio $i$ copia a opinião de $j$.

No caso de 2 opiniões, essa regra lembra a dinâmica de Glauber para temperatura 0, porém com a diferença que o sítio não copia a opinião da maioria de seus vizinhos (isso acontece no chamado modelo do votante majoritário) e sim a opinião de algum de seus vizinhos.

Essa regra de evolução permite estabelecer conexões entre as propriedades do modelo com diferentes quantidades de opiniões, por causa da seguinte transformação: Considere o modelo com $M$ opiniões e monte $m<M$ conjuntos disjuntos $X_{i}$ que possuam todas as opiniões entre 1 e $M$. Ou seja,

$$
X_{i} \cap X_{j}=\varnothing \forall i \neq j \text { e } \cup_{i} X_{i}=\{1, \ldots, M\} .
$$

Se um sítio tiver opinião $\sigma \in X_{i}$, ele passa a ter opinião $\xi_{i}$. Com essa transformação podemos olhar como seria a evolução do modelo considerando, ao invés das opiniões novas $\xi_{i}$, as opiniões antigas subjacentes $\sigma$ : se um sítio tem opinião $\sigma$ e copia a opinião de seu vizinho que tem opinião $\sigma^{\prime}$, ele passa a ter opinião $\sigma^{\prime}\left(\sigma, \sigma^{\prime} \in\{1, \ldots, M\}\right)$. Se, por outro lado, $\sigma \in X_{i}$ e $\sigma^{\prime} \in X_{j}$, então temos, do ponto de vista das opiniões novas, um sítio com opinião $\xi_{i}$ que copia a opinião de um sítio que tem opinião $\xi_{j}$ e passa também a ter opinião $\xi_{j}$, ou seja as regras são as mesmas não importa qual dos 2 conjuntos de opiniões seja usado para descrever a dinâmica. Uma forma menos abstrata de entender isso é considerar que o conjunto original de opiniões trazia informações sobre 2 questões diferentes e no novo conjunto de opiniões nós nos restringimos a uma delas.

Uma consequência disso é que, se as opiniões são sorteadas em uma rede finita com uma probabilidade que independe do sítio (opiniões diferentes podem ser sorteadas com probabilidades diferentes), então a probabilidade de que eventualmente a rede se encontre em um estado de consenso da opinião $\sigma$ é igual à probabilidade com que a opinião $\sigma$ foi sorteada na condição inicial. Para entender esse fato, seja $p_{\sigma}$ a probabilidade de sorteio da opinião $\sigma$. Podemos aproximar os $p_{\sigma}$ tão bem quanto se queira por números racionais $q_{\sigma} / \mathrm{m}$, que somem 1. Então, se considerarmos um modelo com $m$ opiniões sorteadas de forma equiprovável na mesma rede, podemos juntar essas opiniões em grupos e identificar esses grupos com as opiniões originais (o grupo associado com a opinião $\sigma$ deve ter $q_{\sigma}$ dessas $m$ opiniões). Como a rede é finita, eventualmente um estado de consenso é atingido e por simetria, o consenso de cada uma das $m$ opiniões é equiprovável, o que prova o resultado para o modelo com as opiniões originais.

Por essa mesma razão, para analisar a dependência da geometria no ordenamento dos sítios no limite termodinâmico, basta olhar o caso com 2 opiniões. Em um reticulado de 
dimensão $D^{[16]}$, se considerarmos a fração de arestas ativas (que conectam 2 sítios com opiniões diferentes) $\rho(t)$, temos os seguintes comportamentos:

$$
\rho(t) \simeq \begin{cases}t^{-1 / 2} & \text { para } D=1 \\ 1 / \ln (t) & \text { para } D=2 \\ a-b t^{-D / 2} & \text { para } D>2 .\end{cases}
$$

Logo, apenas para $D \leq 2$ surgem grandes domínios ordenados, com só uma opinião. Para dimensões maiores, o sistema cai em estados meta-estáveis, em que o crescimento dos domínios das 2 opiniões, em média, é nulo. Nas redes de Watts-Strogatz e Barabási-Albert vemos um comportamento parecido com o do modelo em um reticulado com dimensionalidade grande; em redes fractais, em que $D$ está entre 1 e 2 , também temos um decaimento de $\rho$ segundo uma lei de potência.

Uma variante do modelo do votante em que a opinião flui de dentro para fora (dinâmica de convencimento) é o modelo Ochrombel. Ele é definido em ${ }^{[30]}$ como:

- Escolha um sítio $i$ de maneira aleatória.

- Todos os vizinhos de $i$ assumem a opinião dele.

Note que esse modelo possui a mesma propriedade do modelo do votante de ter a mesma regra para opiniões isoladas e para grupos de opiniões, valendo o mesmo resultado para a probabilidade de consenso em função da forma como as condições iniciais são escolhidas. Esse modelo só foi estudado em uma rede quadrada e nesse caso, sempre se ordena.

Outra modificação, também conhecida como modelo Ochrombel na literatura é

- Escolha um sítio $i$ de maneira aleatória.

- Um dos vizinhos de $i$, escolhido aleatoriamente, assume a opinião dele.

Interessantemente, para um reticulado essa modificação é indistinguível do modelo do votante.

\section{4 modelo Sznajd}

O principal modelo que iremos estudar é o modelo Sznajd e suas variações. Assim como no modelo do votante, as opiniões dos agentes são números inteiros. Apesar dele possuir diversas variações, grosso modo a dinâmica do modelo Sznajd é a seguinte

- Sorteie um sítio $i$, aleatoriamente.

- Sorteie um vizinho $j$ de $i$, aleatoriamente (todos os vizinhos são sorteados com probabilidade igual). 
- Se $i$ e $j$ concordam, ou seja, se $\sigma_{i}=\sigma_{j}$, então $i$ e $j$ tentam convencer os seus vizinhos.

- Se por outro lado, $i$ e $j$ discordam, nada acontece.

Esse modelo foi originalmente proposto para uma rede unidimensional em ${ }^{[40]}{ }^{1}$, depois foi adaptado para reticulados em ${ }^{[39]}$ e generalizado para uma rede genérica em ${ }^{[7]}$.

Uma das coisas que varia entre essas versões é a forma como que os vizinhos, a serem convencidos, são escolhidos. $\mathrm{Em}^{[39]}$, que define o modelo em um reticulado de dimensão $d$, todos os vizinhos de $i$ e de $j$ ( $4 d-2$ no total) eram convencidos. Já em ${ }^{[46 ; 7]}$ cada vizinho de $i$ é convencido com probabilidade $1 / q_{i}$ e cada vizinho de $j$, com probabilidade $1 / q_{j}$. Na versão que usamos $\mathrm{em}^{[43]}$ (e nesse trabalho), apenas um vizinho de $j$ é escolhido aleatoriamente. O importante de todas essas versões é que o número médio de vizinhos que são convencidos quando um par que concorda é encontrado seja o mesmo, não importando quem são os sítios $i$ e $j(4 d-2,2$ e 1 , respectivamente). Ao longo de todo esse trabalho, o nosso foco será no modelo Sznajd usando regras de confiança. Com essa modificação, as regras passam a ser

- Sorteie um sítio $i$, aleatoriamente.

- Sorteie um vizinho $j$ de $i$, aleatoriamente (todos os vizinhos são sorteados com probabilidade igual).

- Se $i$ e $j$ discordam, nada acontece.

- Por outro lado, se $i$ e $j$ concordam, ou seja, se $\sigma_{i}=\sigma_{j}$, então sorteamos um vizinho $k$ de $j$.

- Se $\sigma^{\prime}$ for a opinião de $i$ e $j$ e $\sigma$ for a opinião de $k$, então $k$ é convencido com probabilidade $p_{\sigma \rightarrow \sigma^{\prime}}$.

Ao contrário do modelo do votante, o limite termodinâmico do modelo Sznajd na ausência de regras de confiança atinge o consenso em reticulados com qualquer número de dimensões, em uma aproximação de campo médio e em redes complexas. O transiente desse modelo, quando simulado em uma rede de Barabási-Albert e com muitas opiniões, exibe uma distribuição do número de sítios com uma dada opinião que tem a forma de uma lei de potência, o que reproduz dados de eleições proporcionais em vários países do mundo ${ }^{[10 ; 7 ; 46]}$.

Também, ao contrário do modelo do votante, quando uma das opiniões é sorteada com probabilidade maior que as outras na condição inicial, a probabilidade que essa opinião domine a rede inteira no estado de consenso tende a 1 , quando o tamanho da rede aumenta. $\mathrm{O}$ argumento de agrupar as opiniões não funciona mais por causa da etapa em que procuramos 2 sítios que concordem, antes da interação de fato.

\footnotetext{
${ }^{1}$ essa versão é equivalente ao modelo do votante ${ }^{[6]}$, por causa que nessa versão, se $i$ e $j$ discordassem, a discordância era propagada. As adaptações feitas posteriormente abandonaram em sua grande maioria a regra de discordância do modelo original
} 
O modelo com confiança limitada foi estudado em redes quadradas por Stauffer ${ }^{[38]}$, que chegou a conclusão que, para 3 opiniões, quase sempre um consenso na opinião intermediária (2) é atingido (quando as opiniões são sorteadas com a mesma probabilidade nas condições iniciais), enquanto que para 4 opiniões ou mais a rede congela quase sempre em um estado em que domínios vizinhos não interagem. Schulze simulou uma versão de vizinhos aleatórios ${ }^{[34]}$ do modelo com confiança limitada, com a mesma condição inicial e chegou nos mesmos resultados que para a rede quadrada no caso de 4 ou mais opiniões. Porém, para 3 opiniões, o consenso na opinião intermediária era atingido em $50 \%$ das vezes e em $50 \%$ das vezes uma coexistência entre as opiniões extremistas (1 e 3) era atingido. Nos próximos capítulos apresentamos uma representação do modelo com espaços de fase e no capítulo 5 mostraremos com mais detalhes que essas diferenças são causadas pela condição inicial que ambos os autores usaram e pelo fato que as bacias de atração dos 2 atratores (consenso em 2 e coexistência de 1 com 3 ) sofrem uma ligeira mudança, por efeitos de rede.

\subsection{O modelo $q$-votante e variações do modelo Sznajd}

Podemos obter generalizações tanto do modelo do votante quanto do modelo Sznajd variando o tamanho do grupo que se forma para convencer um sítio. No modelo $q$-votante, ao invés de um sítio checar a opinião de um de seus vizinhos, $q$ vizinhos são levados em conta e se eles concordarem o sítio assume a opinião desse grupo. A versão original do modelo, definida em ${ }^{[9]}$, também introduzia uma probabilidade do sítio mudar de opinião espontaneamente se os $q$ sítios não concordassem entre si. Mais precisamente as regras são

- Escolha um sítio $i$ aleatoriamente.

- Sorteie $q$ sítios $j_{1}, \ldots, j_{q}$, tais que $j_{k} \in \Gamma_{i}$ e onde repetições são permitidas.

- Se todos os sítios $j_{k}$ concordarem, então $i$ copia a opinião deles.

- Se não, com probabilidade $\epsilon$ (um parâmetro do modelo) o sítio $i$ assume uma opinião aleatória e com probabilidade $1-\epsilon$, ele mantém a sua mesma opinião.

Uma modificação análoga pode ser feita no modelo Sznajd, em que primeiro buscamos um grupo de tamanho $q$ e depois sorteamos o sítio a ser convencido, ou seja:

- Escolha uma sequência de $q$ sítios $j_{1}, \ldots, j_{q}$, em que $j_{1}$ é escolhido aleatoriamente entre todos os sítios da rede e $j_{k+1}$ é escolhido aletoriamente dentre os vizinhos de $j_{k}$.

- Se eles não tiverem todos a mesma opinião, nada acontece.

- Se eles concordarem, escolha $i$, um vizinho de $j_{q}$. O grupo então convence o sítio $i$. 
$\mathrm{Na}$ aproximação de campo médio, os 2 modelos são equivalentes (tomando $\epsilon=0$ no $q$-votante) e enquanto essa modificação não causa grandes alterações para o modelo Sznajd (a maior é que quando as opiniões aparecem em proporções parecidas a dinâmica fica consideravelmente mais lenta, já que se torna mais difícil montar um grupo com $q$ sítios que concordem entre si), o modelo $q$-votante só se comporta como o modelo do votante original para valores precisos de $q$ e $\epsilon$.

\subsection{O Modelo Deffuant e o modelo HK}

O modelo Deffuant ${ }^{[11]}$ é um modelo de opiniões contínuas, ao contrário dos modelos votante e Sznajd. Além disso, a dinâmica dele é baseada na idéia de homogenização, com agentes discutindo e buscando um compromisso entre suas opiniões originais. Outra diferença é que a confiança limitada é um ingrediente essencial (senão o modelo se reduz a uma dinâmica de difusão). Em um modelo de opiniões contínuas a idéia de opiniões iguais ou da maioria não é tão útil. Ao invés disso, uma tolerância a opiniões diferentes é definida, ou seja, temos um parâmetro $\epsilon$ definindo essa tolerância e 2 sítios só interagem de fato se a diferença entre as suas opiniões for de no máximo $\epsilon$. As regras do modelo em detalhe são:

- Escolha um sítio $i$ aleatoriamente.

- Escolha um vizinho $j$ de $i$.

- Se as opiniões dos dois, $\sigma_{i}$ e $\sigma_{j}$ forem tais que $\left|\sigma_{i}-\sigma_{j}\right| \leq \epsilon$, eles interagem.

- A interação dos sítios depende de um parâmetro $\mu$ :

$$
\left\{\begin{array}{l}
\sigma_{i}:=(1-\mu) \sigma_{i}+\mu \sigma_{j} \\
\sigma_{j}:=(1-\mu) \sigma_{j}+\mu \sigma_{i} .
\end{array}\right.
$$

A interpretação dos parâmetros é simples: $\epsilon$ mede a tolerância dos agentes a pontos de vista distintos e $\mu$ (entre 0 e $1 / 2$ ) mede o quanto eles estão dispostos a um compromisso com uma outra opinião que tolerem. Após algum tempo, os sítios formam domínios em que todos os sítios estão a uma distância menor que $\epsilon$ de uma certa opinião e que não podem mais interagir com sítios fora desse domínio, de forma que o modelo evolui para uma situação em que os sítios de cada domínio eventualmente atingem consenso entre si, mas os diferentes domínios não interagem. Em geral, os tamanhos e a quantidade desses domínios dependem de $\epsilon$ e a dependência com $\mu$ afeta apenas a velocidade da dinâmica (porém há uma dependência em $\mu$ para as características dos domínios, para valores baixos de $\mu$ ). Em um grafo completo, o número de domínios é inversamente proporcional a $\epsilon$ e em redes complexas em geral, além dessa dependência, temos que o número de domínios é proporcional ao número de sítios.

O modelo Hegselmann-Krause $(\mathrm{HK})^{[21]}$ é bem parecido em alguns aspectos com o modelo Deffuant. Também temos opiniões contínuas e a regra de confiança limitada, porém as regras de interação são diferentes e se baseiam na idéia de adaptação: 
- Escolha um sítio $i$.

- Seja $X_{i}$, o conjunto de vizinhos compatíveis com $i, X_{i}=\left\{j \in \Gamma_{i}\right.$ tal que $\left|\sigma_{j}-\sigma_{i}\right| \leq$ $\epsilon\}$.

- $i$ Assume a opinião média dos sítios em $X_{i}$.

Apesar das semelhanças com o modelo Deffuant, esse modelo tem simulações mais demoradas e também foi menos estudado. Apesar disso, o padrão qualitativo dos estados estacionários é bem parecido nos 2 modelos, porém com uma dependência no grau médio do grafo em que o modelo é simulado ${ }^{[15]}$.

\subsection{O modelo Axelrod}

Esse modelo foi proposto em ${ }^{[3]}$ para explicar a formação de culturas e tentar resolver a questão de como interações que localmente aumentam a similaridade são capazes de criar diferenças culturais persistentes. A ideia por trás do modelo é que os agentes devem interagir de maneira que a similaridade entre eles propicie uma interação (uma forma de confiança limitada) e uma interação tenda a aumentar a similaridade, porém agentes que não sejam similares, não interagem e portanto sustentam suas diferenças. As opiniões são representadas por vetores de inteiros $\sigma_{i}=\left(c_{i 1}, c_{i 2}, \ldots, c_{i F}\right)$ em que cada componente é um número inteiro que pode variar entre 1 e $Q$. As regras do modelo em detalhe são:

- Escolha de maneira aleatória um arco $(i, j)$.

- Os sítios interagem com probabilidade igual a $\frac{1}{F} \sum_{k=1}^{F} \delta_{c_{i k}, c_{j k}}$.

- Na interação, escolha aleatoriamente um inteiro $s$ entre 1 e $Q$, tal que $c_{i s} \neq c_{j s}$ (não há interação se $\sigma_{i}=\sigma_{j}$ ). $c_{i s}$ passa a valer $c_{j s}$.

Note que a escolha de $(i, j)$ e de $(j, i)$ tem a mesma probabilidade, logo a discussão da diferença entre convencimento e adaptação é irrelevante nesse caso. Além disso, a probabilidade de interação entre $i$ e $j$ pode ser reescrita como

$$
1-\frac{\left\|\sigma_{i}-\sigma_{j}\right\|}{F},
$$

onde a norma é derivada a partir da distância de Hamming (o que deixa claro o papel da confiança limitada)

O modelo apresenta comportamentos diferentes nas redes de Watts-Strogatz e na rede Barabási-Albert ${ }^{[26]}$. Na primeira (que engloba também reticulados e grafos aleatórios para $p$ igual a 0 e 1 respectivemente), se considerarmos uma rede quadrada e redirecionarmos as arestas com probabilidade $p$, podemos analisar o que acontece quando variamos o valor 
de $Q$, usando como parâmetro de ordem a fração da rede ocupada pela maior componente $s_{\text {max }}$ (um conjunto conexo de sítios que possuem todos a mesma opinião). O modelo exibe uma transição de fase de primeira ordem, quando variamos $Q$. Para $Q<Q_{c}(p)$, o modelo se ordena e quase todos os sítios pertencem a mesma componente enquanto que para $Q>$ $Q_{c}(p)$, ele atinge um estado congelado desordenado. Na vizinhança de $Q_{c}(p)$, o modelo exibe biestabilidade (quando $N$ não é grande) e pode cair tanto no estado ordenado, quanto no desordenado. O comportamento de $Q_{c}$ obedece $Q_{c}(p)-Q_{c}(0) \simeq p^{0,4}$ e, a partir de $Q_{c}(1)$, o modelo sempre termina na fase desordenada.

Já na rede Barabási-Albert, a relação entre $Q$ e $N$, para que o modelo exiba ordem ou desordem, é diferente. Essa relação pode ser obtida reescalando $Q$ e colapsando os gráficos de $\left\langle s_{\max }>(Q)\right.$, de forma que obtemos $\left\langle s_{\max }>=f\left(Q N^{-0,39}\right)\right.$, com $f(0)=1$ e $\lim _{x \rightarrow \infty} f(x)=0$. Segue que, para um $Q$ fixo, o modelo sempre se ordena no limite termodinâmico. 


\section{Capítulo 3}

\section{Dedução das equações de campo médio a partir de cadeias de Markov}

"In mathematics you don't understand things. You just get used to them."

- John von Neumann

\subsection{Um exemplo simples de cadeia de Markov}

Antes de fazermos as deduções das equações do modelo Sznajd na aproximação de campo médio, iremos usar o movimento browniano como um exemplo para expor algumas das idéias básicas em um contexto mais simples.

Uma cadeia de Markov é um processo estocástico com tempo discreto, em que o estado seguinte do sistema só depende do estado atual do sistema (o que é equivalente ao estado seguinte depender de apenas de um número finito fixo de estados anteriores, redefinindo de maneira conveniente quais são os estados do modelo), ou seja, o sistema não tem memória. Estamos interessados em particular em como a probabilidade do sistema se encontrar em um determinado estado evolui no tempo. Ou seja, se $S$ for o conjunto de estados do sistema (que pode ser infinito), então estamos interessados nas funções $P(s ; t)$, onde $P(s ; t)$ dá a probabilidade do sistema se encontrar no estado $s \in S$ no instante de tempo $t$. Como o estado no tempo $t+1$ só depende do estado no tempo $t$, devem existir probabilidades $\mathcal{P}_{s \rightarrow s^{\prime}}$ que dão a probabilidade do sistema ir do estado $s$ no tempo $t$ até o estado $s^{\prime}$ no tempo $t+1$. Considerando como independentes os eventos "o sistema está no estado s no tempo $t$ ", podemos calcular $P(s ; t+1)$ contabilizando para todos os estados possíveis as transições para $s$, ponderadas pelas probabilidades no tempo $t$, o que nos leva a chamada equação mestra:

$$
P(s ; t+1)=\sum_{s \in S} P\left(s^{\prime} ; t\right) \mathcal{P}_{s^{\prime} \rightarrow s}
$$


No movimento Browniano que iremos considerar, temos uma partícula em uma dimensão que, com probabilidade $p \leq 1 / 2$, anda uma distância 1 (em alguma unidade conveniente) em uma direção, com probabilidade $p$, ela anda 1 na direção oposta e com probabilidade $1-2 p$, ela permanece no mesmo lugar. O estado do sistema é a posição da partícula e as transições são dadas por

$$
\mathcal{P}_{s^{\prime} \rightarrow s}= \begin{cases}p, & \text { se } s^{\prime}=s-1 \\ 1-2 p, & \text { se } s^{\prime}=s \\ p, & \text { se } s^{\prime}=s+1 \\ 0, & \text { se }\left|s^{\prime}-s\right|>1\end{cases}
$$

de forma que a equação mestra é

$$
P(s ; t+1)=p(P(s+1 ; t)+P(s-1 ; t))+(1-2 p) P(s ; t) .
$$

Um tratamento comum para a equação 3.1.3, é reescrevê-la usando um laplaciano discretizado:

$$
\begin{gathered}
P(s ; t+1)-P(s ; t)=p(P(s+1 ; t)-P(s ; t)-(P(s ; t)-P(s-1 ; t))) \Rightarrow \\
\Rightarrow \Delta_{t} P=p \Delta_{s}^{2} P
\end{gathered}
$$

e aproximar o resultado por uma equação de difusão (trocar os operadores de diferenças por operadores diferenciais). No nosso caso, iremos estudar principalmente a evolução no tempo de grandezas médias no tempo. Como a equação 3.1.3 é uma difusão pura, nada de interessante acontece com $\bar{s}$. Considere por outro lado um movimento Browniano enviesado, com transições

$$
\mathcal{P}_{s^{\prime} \rightarrow s}= \begin{cases}p, & \text { se } s^{\prime}=s-1 \\ 1-p-q, & \text { se } s^{\prime}=s \\ q, & \text { se } s^{\prime}=s+1 \\ 0, & \text { se }\left|s^{\prime}-s\right|>1\end{cases}
$$

onde $p+q \leq 1$ e $p>q$. A equação mestra associada é

$$
\begin{gathered}
P(s ; t+1)=q P(s+1 ; t)+p P(s-1 ; t)+(1-p-q) P(s ; t)= \\
=p(P(s+1 ; t)+P(s-1 ; t))+(1-2 p) P(s ; t)+(q-p)(P(s+1 ; t)-P(s ; t)) \Rightarrow \\
\Rightarrow \Delta_{t} P=p \Delta_{s}^{2} P+(q-p) \Delta_{s} P
\end{gathered}
$$

que corresponde a uma difusão com drift e $\log o \bar{s}$ evolui no tempo.

Definindo de maneira mais precisa essa média:

$$
\bar{s}(t)=\sum_{s \in \mathbb{Z}} s P(s ; t)
$$


e a evolução temporal pode ser obtida a partir da equação mestra

$$
\begin{gathered}
\bar{s}(t+1)=\sum_{s \in \mathbb{Z}} s P(s ; t+1)=\sum_{s \in \mathbb{Z}} s(q P(s+1 ; t)+p P(s-1 ; t)+(1-p-q) P(s ; t))= \\
=\sum_{s \in \mathbb{Z}} q(s-1) P(s ; t)+p(s+1) P(s ; t)+s(1-p-q) P(s ; t)= \\
=q(\bar{s}-1)+p(\bar{s}+1)+\bar{s}(1-p-q) \Rightarrow \Delta_{t} \bar{s}=p-q .
\end{gathered}
$$

\subsection{Notações}

Antes de obter as equações mestras dos modelos estudados com uma regra de confiança $p_{\sigma \rightarrow \sigma^{\prime}}$, iremos introduzir algumas notações.

O estado do sistema é dado pelas opiniões de todos os sítios e denotado $\vec{\sigma}=\left(\sigma_{1}, \sigma_{2}, \ldots, \sigma_{N}\right)$. Para denotar modificações do estado $\vec{\sigma}$, vamos usar ( " $\left.\vec{\sigma} ", i_{1}: \xi_{1}, \ldots, i_{m}: \xi_{m}\right)$, para denotar o estado em que cada sítio $j$ tem opinião $\sigma_{j}$, exceto os sítios $i_{k}$, que tem opiniões $\xi_{k}$. De forma parecida, $\left(i_{1}: \xi_{1}, \ldots, i_{m}: \xi_{m}\right)$ denota o evento em que os sítios $i_{k}$ tem opinião $\xi_{k}$, independente da opinião dos outros sítios. Logo, se temos, por exemplo, 5 sítios e $\vec{\sigma}=(a, b, c, d, e)$, segue que (" $\vec{\sigma}$ ", $2: f, 4: g)$ denota o estado $(a, f, c, g, e)$ e $(2: f, 4: g)$ denota todos os estados $(x, f, y, g, z)$, para todas as possibilidades de $x, y$ e $z$.

$P_{\tau}(X)$ vai denotar a probabilidade que o evento $X$ ocorra na rede, na iteração $\tau$ (se não houver ambiguidade $\tau$ pode ser omitido). No nosso exemplo anterior, $P_{\tau}(\vec{\sigma})$ é a probabilidade que a rede se encontre no estado $(a, b, c, d, e)$ na iteração $\tau$, ou seja, $P_{\tau}(a, b, c, d, e) \mathrm{e}$ $P_{\tau}(2: f, 4: g)$ é a probabilidade que o sítio 2 tenha opinião $f$ e o sítio 4 tenha opinião $g$, ou seja,

$$
P_{\tau}(2: f, 4: g)=\sum_{x, y, z} P_{\tau}(x, f, y, g, z) .
$$

Iremos denotar o somatório

$$
\sum_{\sigma_{1}} \sum_{\sigma_{2}} \ldots \sum_{\sigma_{N}}(\ldots)
$$

por

$$
\sum_{\{\vec{\sigma}\}}(\ldots)
$$

(ou seja, estamos somando sobre todos os estados) e o somatório

$$
\sum_{\sigma_{c_{1}}} \sum_{\sigma_{c_{2}}} \ldots \sum_{\sigma_{c_{n}}}(\ldots)
$$


onde $c_{1}, c_{2}, \ldots, c_{n}$ são os inteiros entre 1 e $N$ tal que alguma função $f:\{1, \ldots, N\} \rightarrow$ \{verdadeiro, falso $\}$ é verdadeira, por

$$
\sum_{\substack{\left\{\sigma_{l}\right\} \\ f(l)}}(\ldots)
$$

Por exemplo,

$$
\sum_{\substack{\left\{\sigma_{l}\right\} \\ l \leq 4}}(\ldots)=\sum_{\sigma_{1}} \sum_{\sigma_{2}} \sum_{\sigma_{3}} \sum_{\sigma_{4}}(\ldots)
$$

Finalmente, se $A$ é alguma expressão booleana, $\delta_{A}$ vale 0 se $A$ é falsa e 1 se $A$ é verdadeira (de forma parecida com a notação de Iverson). $\delta_{a, b, c, \ldots, d}$ denota o mesmo que $\delta_{(a=b=c=\ldots=d)}$. Relembramos que $\Gamma_{i}$ é o conjunto de vizinhos de $i$ e $q_{i}$ é o número de vizinhos de $i$ (ver a seção 1.2).

Vamos deduzir a equação de campo médio dos modelos Sznajd, votante e Ochrombel. Vamos começar com a dedução do modelo do votante e fazer adaptações na dedução para o modelo Ochrombel, de forma a fixar algumas idéias primeiro com casos mais simples. A idéia é primeiro escrever a equação mestra, obtida fazendo uma contagem de todos os processos permitidos pelo modelo, para ter uma equação de evolução temporal para $P_{\tau}(\vec{\sigma})$, a distribuição de probabilidades do estado da rede, no tempo $\tau$. A partir daí, a transformamos em uma equação de diferenças, chegando em uma expressão para $\Delta P(\vec{\sigma})$, a variação na probabilidade de encontrar o sistema no estado $\vec{\sigma}$ no passo de tempo em questão ( $\tau$ passa a ser omitido). Para cada sítio $i$, fazemos uma soma dos $\Delta P(\vec{\sigma})$, de forma a obter a variação da distribuição marginal $P(i: \sigma)$ a cada passo de tempo, que pode depender de distribuições marginais envolvendo mais de um sítio (como $P\left(i: \sigma, j: \sigma^{\prime}, k: \sigma^{\prime \prime}\right)$ ). Fazemos então uma aproximação que pode ser interpretada como uma aproximação de campo médio, quando usamos um grafo completo, de forma que as equações fechem (ou seja, tal que a evolução temporal da distribuição marginal envolvendo um sítio só depende das outras distribuições marginais de um sítio). Finalmente, para deduzir as versões de campo médio, colocamos o modelo em um grafo completo e tomamos o limite termodinâmico, o que transforma a equação de diferenças em uma equação diferencial.

Por simplicidade vamos assumir um grafo não-direcionado conexo e com mais de um vértice (de forma que todo sítio tem algum vizinho) para ser a geometria do modelo.

\subsection{O modelo do votante}

A equação mestra do modelo do votante é dada por 


$$
P_{\tau+1}(\vec{\sigma})=\frac{1}{N} \sum_{i} \frac{1}{q_{i}} \sum_{j \in \Gamma_{i}}(\underbrace{\left(1-p_{\sigma_{i} \rightarrow \sigma_{j}}\right) P_{\tau}(\vec{\sigma})}_{A}+\sum_{\sigma^{\prime}} \underbrace{P_{\tau}\left(“ \vec{\sigma} ", i: \sigma^{\prime}\right) p_{\sigma^{\prime} \rightarrow \sigma_{j}} \delta_{\sigma_{i}, \sigma_{j}}}_{B}) .
$$

Em todos os termos da equação, $i$ é o primeiro sítio a ser escolhido e $j$ é o segundo, logo por causa das regras do modelo, com probabilidade $p_{\sigma_{i} \rightarrow \sigma_{j}}$, o sítio $i$ copia a opinião de $j$, caso contrário, o estado não muda. Para obter a equação 3.3.1, é necessário contar todas as probabilidades de transição de algum estado $\vec{\sigma}^{\prime}$ para o estado $\vec{\sigma}$, ponderando a soma por $P_{\tau}\left(\vec{\sigma}^{\prime}\right)$. O termo $A$ contabiliza os casos em que o estado não muda, enquanto que $B$ contabiliza todas as mudanças de estados em que $\sigma^{\prime}$ era a opinião anterior de $i$, ou seja, $\vec{\sigma}^{\prime}=\left(" \vec{\sigma} ", i: \sigma^{\prime}\right)$. Esse termo é proporcional a $\delta_{\sigma_{i}, \sigma_{j}}$, pois os únicos estados que podem ser atingidos a partir de uma mudança em que $i$ e $j$ interagiram são aqueles em que $i$ e $j$ concordam. Vale lembrar que $\sigma_{i}$ e $\sigma_{j}$ são as opiniões no estado $\vec{\sigma}$, ou seja, depois da interação entre os 2 sítios.

Como

$$
\frac{1}{N} \sum_{i} \frac{1}{q_{i}} \sum_{j \in \Gamma_{i}} P_{\tau}(\vec{\sigma})=P_{\tau}(\vec{\sigma})
$$

então a equação 3.3.1 pode ser facilmente reescrita como

$$
\Delta P(\vec{\sigma})=\frac{1}{N} \sum_{i} \frac{1}{q_{i}} \sum_{j \in \Gamma_{i}}\left(-p_{\sigma_{i} \rightarrow \sigma_{j}} P(\vec{\sigma})+\sum_{\sigma^{\prime}} P\left(“ \vec{\sigma} ", i: \sigma^{\prime}\right) p_{\sigma^{\prime} \rightarrow \sigma_{j}} \delta_{\sigma_{i}, \sigma_{j}}\right) .
$$

Para fazer a marginalização da distribuição vamos usar

$$
P(s: \sigma)=\sum_{\{\vec{\sigma}\}} P(\vec{\sigma}) \delta_{\sigma_{s}, \sigma} .
$$

Aplicando na equação 3.3 .3 chegamos em

$$
\begin{gathered}
\Delta P(s: \sigma)=\underbrace{-\frac{1}{N} \sum_{i} \frac{1}{q_{i}} \sum_{j \in \Gamma_{i}} \sum_{\{\vec{\sigma}\}} \delta_{\sigma_{s}, \sigma} p_{\sigma_{i} \rightarrow \sigma_{j}} P(\vec{\sigma})}_{C}+ \\
+\underbrace{\frac{1}{N} \sum_{i} \frac{1}{q_{i}} \sum_{j \in \Gamma_{i}} \sum_{\{\vec{\sigma}\}} \delta_{\sigma_{s}, \sigma} \sum_{\sigma^{\prime}} P\left(“ \vec{\sigma} ", i: \sigma^{\prime}\right) p_{\sigma^{\prime} \rightarrow \sigma_{j}} \delta_{\sigma_{i}, \sigma_{j}}}_{D} .
\end{gathered}
$$

Iremos primeiro analisar o termo $D$. A soma em $\vec{\sigma}$ pode ser sempre separada da seguinte forma: 


$$
\sum_{\{\vec{\sigma}\}}(\ldots)=\sum_{\substack{\left\{\sigma_{l}\right\} \\ l \neq i}} \sum_{\sigma_{i}}(\ldots)
$$

Como $P\left(" \vec{\sigma}\right.$ ", $\left.i: \sigma^{\prime}\right)$ não depende de $\sigma_{i}$ (lembramos novamente que $\sigma_{i}$ seria o estado de $i$ depois do convencimento e o que entra no cálculo é o estado anterior), então a soma em $D$ pode ser reordenada como

$$
D=\frac{1}{N} \sum_{i} \frac{1}{q_{i}} \sum_{j \in \Gamma_{i}} \sum_{\substack{\left\{\sigma_{l}\right\} \\ l \neq i}} \sum_{\sigma^{\prime}} P\left(“ \vec{\sigma} ", i: \sigma^{\prime}\right) p_{\sigma^{\prime} \rightarrow \sigma_{j}} \sum_{\sigma_{i}} \delta_{\sigma_{s}, \sigma} \delta_{\sigma_{i}, \sigma_{j}} .
$$

Chamamos a atenção para o fato que na soma

$$
\sum_{\sigma_{i}} \delta_{\sigma_{s}, \sigma} \delta_{\sigma_{i}, \sigma_{j}}
$$

podemos ter resultados diferentes se houverem repetições entre os sítios $i, j$ e $s$. Como $j \in \Gamma_{i}$, segue que $i \neq j$ sempre, porém se $i=s$ temos

$$
\sum_{\sigma_{i}} \delta_{\sigma_{i}, \sigma_{j}, \sigma}
$$

enquanto que se $i \neq s$ temos

$$
\delta_{\sigma_{s}, \sigma} \sum_{\sigma_{i}} \delta_{\sigma_{i}, \sigma_{j}}
$$

ou seja

$$
\sum_{\sigma_{i}} \delta_{\sigma_{s}, \sigma} \delta_{\sigma_{i}, \sigma_{j}}=\delta_{i=s} \delta_{\sigma_{j}, \sigma}+\delta_{i \neq s} \delta_{\sigma_{s}, \sigma}
$$

A diferença entre os casos $i=s$ e $i \neq s$, que aparece na equação 3.3.7, é responsável por fazer todos os termos que não modificam $s$ se cancelarem em 3.3.5. Voltando a nossa expressão para $D$ temos

$$
D=\frac{1}{N} \sum_{i} \frac{1}{q_{i}} \sum_{j \in \Gamma_{i}} \sum_{\substack{\left\{\sigma_{l}\right\} \\ l \neq i}} \sum_{\sigma^{\prime}} P\left(" \vec{\sigma} ", i: \sigma^{\prime}\right) p_{\sigma^{\prime} \rightarrow \sigma_{j}}\left(\delta_{i=s} \delta_{\sigma_{j}, \sigma}+\delta_{i \neq s} \delta_{\sigma_{s}, \sigma}\right) .
$$

Agora chamamos a atenção para o fato que $D$ não depende de $\sigma_{i}$ (na verdade, depois da marginalização o estado $\vec{\sigma}$ ao qual $\sigma_{i}$ faz parte se tornou apenas uma varíavel muda) e que logo podemos fazer a mudança de varíaveis $\sigma^{\prime}=\sigma_{i}$, porém ( " $\left.\vec{\sigma} ", i: \sigma_{i}\right)=\vec{\sigma}$, de forma que a nossa expressão para $D$ passa a ser 


$$
D=\frac{1}{N} \sum_{i} \frac{1}{q_{i}} \sum_{j \in \Gamma_{i}} \sum_{\{\vec{\sigma}\}} P(\vec{\sigma}) p_{\sigma_{i} \rightarrow \sigma_{j}}\left(\delta_{i=s} \delta_{\sigma_{j}, \sigma}+\delta_{i \neq s} \delta_{\sigma_{s}, \sigma}\right) .
$$

Mas então, juntando com $C$ (equação 3.3.5), todos os termos tais que $i \neq s$ se cancelam, pois

$$
C=-\frac{1}{N} \sum_{i} \frac{1}{q_{i}} \sum_{j \in \Gamma_{i}} \sum_{\{\vec{\sigma}\}} P(\vec{\sigma}) p_{\sigma_{i} \rightarrow \sigma_{j}}\left(\delta_{i=s} \delta_{\sigma_{s}, \sigma}+\delta_{i \neq s} \delta_{\sigma_{s}, \sigma}\right),
$$

logo a equação 3.3 .5 fica

$$
\begin{gathered}
\Delta P(s: \sigma)=\frac{1}{N} \sum_{i} \frac{1}{q_{i}} \sum_{j \in \Gamma_{i}} \sum_{\{\vec{\sigma}\}} \delta_{i, s} P(\vec{\sigma}) p_{\sigma_{i} \rightarrow \sigma_{j}}\left(\delta_{\sigma_{j}, \sigma}-\delta_{\sigma_{s}, \sigma}\right)= \\
=\frac{1}{N q_{s}} \sum_{j \in \Gamma_{s}} \sum_{\{\vec{\sigma}\}} P(\vec{\sigma}) p_{\sigma_{s} \rightarrow \sigma_{j}}\left(\delta_{\sigma_{j}, \sigma}-\delta_{\sigma_{s}, \sigma}\right)= \\
=\frac{1}{N q_{s}} \sum_{j \in \Gamma_{s}} \sum_{\sigma_{j}, \sigma_{s}} P\left(j: \sigma_{j}, s: \sigma_{s}\right) p_{\sigma_{s} \rightarrow \sigma_{j}}\left(\delta_{\sigma_{j}, \sigma}-\delta_{\sigma_{s}, \sigma}\right) \Rightarrow \\
\Delta P(s: \sigma)=\frac{1}{N q_{s}} \sum_{j \in \Gamma_{s}} \sum_{\sigma^{\prime}}(\underbrace{P\left(j: \sigma, s: \sigma^{\prime}\right) p_{\sigma^{\prime} \rightarrow \sigma}}_{E}-\underbrace{P\left(j: \sigma^{\prime}, s: \sigma\right) p_{\sigma \rightarrow \sigma^{\prime}}}_{F}) .
\end{gathered}
$$

A interpretação dos 2 termos $E$ e $F$ é clara. $E$ é o termo contabilizando a transição do sítio $s$ da opinião $\sigma^{\prime}$ para a opinião $\sigma$ e $F$ contabiliza o processo inverso. É interessante notar que na ausência de regras de confiança, a equação 3.3 .10 pode ser simplificada um pouco mais:

$$
\Delta P(s: \sigma)=\frac{1}{N q_{s}} \sum_{j \in \Gamma_{s}}(P(j: \sigma)-P(s: \sigma))=\frac{1}{N}\left(\langle P(j: \sigma)\rangle_{j \in \Gamma_{s}}-P(s: \sigma)\right) .
$$

Nesse caso, as equações fecham e além disso, definindo o vetor $\vec{P}_{\sigma} \equiv(P(1: \sigma), \ldots, P(N: \sigma))$ vem que a equação 3.3.11 pode ser reescrita como

$$
\Delta \vec{P}_{\sigma}=\frac{1}{N} \mathbb{L}_{G} \vec{P}_{\sigma}
$$

onde $\mathbb{L}_{G}$ é o operador laplaciano do grafo que forma a geometria do modelo.

Voltando à equação 3.3.10, para chegar nas equações de campo médio que usamos mais adiante, primeiro fazemos a aproximação $P\left(i: \sigma, j: \sigma^{\prime}\right)=P(i: \sigma) P\left(j: \sigma^{\prime}\right)$, quando $i$ e $j$ são vizinhos: 


$$
\Delta P(s: \sigma)=\frac{1}{N q_{s}} \sum_{j \in \Gamma_{s}} \sum_{\sigma^{\prime}}\left(P(j: \sigma) P\left(s: \sigma^{\prime}\right) p_{\sigma^{\prime} \rightarrow \sigma}-P\left(j: \sigma^{\prime}\right) P(s: \sigma) p_{\sigma \rightarrow \sigma^{\prime}}\right),
$$

depois colocamos o modelo em um grafo completo, chegando em

$$
\begin{aligned}
& \Delta P(s: \sigma)=\frac{1}{N(N-1)} \sum_{j \neq s} \sum_{\sigma^{\prime}}\left(P(j: \sigma) P\left(s: \sigma^{\prime}\right) p_{\sigma^{\prime} \rightarrow \sigma}-P\left(j: \sigma^{\prime}\right) P(s: \sigma) p_{\sigma \rightarrow \sigma^{\prime}}\right)= \\
& =\frac{1}{N^{2}} \sum_{j} \sum_{\sigma^{\prime}}\left(P(j: \sigma) P\left(s: \sigma^{\prime}\right) p_{\sigma^{\prime} \rightarrow \sigma}-P\left(j: \sigma^{\prime}\right) P(s: \sigma) p_{\sigma \rightarrow \sigma^{\prime}}\right)+\mathcal{O}\left(\frac{1}{N^{2}}\right) .
\end{aligned}
$$

Finalmente, definindo $\eta_{\sigma}$ como sendo o valor esperado da proporção de sítios com opinião $\sigma$, como a proporção de sítios com opinião $\sigma$ no estado $\vec{\sigma}$ é dada por

$$
\mathcal{P}_{\sigma}(\vec{\sigma})=\frac{1}{N} \sum_{i} \delta_{\sigma_{i}, \sigma}
$$

então

$$
\eta_{\sigma}=\sum_{\{\vec{\sigma}\}} \mathcal{P}_{\sigma}(\vec{\sigma}) P(\vec{\sigma})=\frac{1}{N} \sum_{i} \sum_{\{\vec{\sigma}\}} \delta_{\sigma_{i}, \sigma} P(\vec{\sigma})=\frac{1}{N} \sum_{i} P(i: \sigma)
$$

e substituindo em 3.3.14, temos

$$
\begin{gathered}
\Delta \eta_{\sigma}=\frac{1}{N^{3}} \sum_{j, s} \sum_{\sigma^{\prime}}\left(P(j: \sigma) P\left(s: \sigma^{\prime}\right) p_{\sigma^{\prime} \rightarrow \sigma}-P\left(j: \sigma^{\prime}\right) P(s: \sigma) p_{\sigma \rightarrow \sigma^{\prime}}\right)+\mathcal{O}\left(\frac{1}{N^{2}}\right)= \\
=\frac{1}{N} \sum_{\sigma^{\prime}} \eta_{\sigma} \eta_{\sigma^{\prime}}\left(p_{\sigma^{\prime} \rightarrow \sigma}-p_{\sigma \rightarrow \sigma^{\prime}}\right)+\mathcal{O}\left(\frac{1}{N^{2}}\right) .
\end{gathered}
$$

A equação 3.3.16 é o mapa obtido quando integramos o seguinte fluxo usando o método de Euler e um passo $1 / N$ :

$$
\dot{\eta}_{\sigma}=\sum_{\sigma^{\prime}} \eta_{\sigma} \eta_{\sigma^{\prime}}\left(p_{\sigma^{\prime} \rightarrow \sigma}-p_{\sigma \rightarrow \sigma^{\prime}}\right)
$$

que é a equação de campo médio do modelo do votante. 


\subsection{O modelo Ochrombel}

A diferença principal entre o caso do modelo do votante e do modelo Ochrombel é que os papéis do primeiro e segundo sítios escolhidos se invertem. A equação mestra é dada por

$$
P_{\tau+1}(\vec{\sigma})=\frac{1}{N} \sum_{i} \frac{1}{q_{i}} \sum_{j \in \Gamma_{i}}(\underbrace{\left(1-p_{\sigma_{j} \rightarrow \sigma_{i}}\right) P_{\tau}(\vec{\sigma})}_{A}+\sum_{\sigma^{\prime}} \underbrace{P_{\tau}\left(“ \vec{\sigma} ", j: \sigma^{\prime}\right) p_{\sigma^{\prime} \rightarrow \sigma_{i}} \delta_{\sigma_{i}, \sigma_{j}}}_{B})
$$

e as interpretações dos termos $A$ e $B$ são as mesmas que para o modelo do votante. Novamente, chegamos em $\Delta P(\vec{\sigma})$ através da equação 3.3 .2

$$
\Delta P(\vec{\sigma})=\frac{1}{N} \sum_{i} \frac{1}{q_{i}} \sum_{j \in \Gamma_{i}}\left(-p_{\sigma_{j} \rightarrow \sigma_{i}} P(\vec{\sigma})+\sum_{\sigma^{\prime}}\left(" \vec{\sigma} ", j: \sigma^{\prime}\right) p_{\sigma^{\prime} \rightarrow \sigma_{i}} \delta_{\sigma_{i}, \sigma_{j}}\right) .
$$

Novamente marginalizamos a distribuição e chegamos em

$$
\begin{gathered}
\Delta P(s: \sigma)=\underbrace{-\frac{1}{N} \sum_{i} \frac{1}{q_{i}} \sum_{j \in \Gamma_{i}} \sum_{\{\vec{\sigma}\}} \delta_{\sigma_{s}, \sigma} p_{\sigma_{j} \rightarrow \sigma_{i}} P(\vec{\sigma})}_{C}+ \\
+\underbrace{\frac{1}{N} \sum_{i} \frac{1}{q_{i}} \sum_{j \in \Gamma_{i}} \sum_{\{\vec{\sigma}\}} \delta_{\sigma_{s}, \sigma} \sum_{\sigma^{\prime}} P\left(“ \vec{\sigma} », j: \sigma^{\prime}\right) p_{\sigma^{\prime} \rightarrow \sigma_{i}} \delta_{\sigma_{i}, \sigma_{j}}}_{D} .
\end{gathered}
$$

Para tratar $D$ separamos a soma em $\sigma_{j}$ do resto da soma em $\{\vec{\sigma}\}$, chegando em

$$
D=\frac{1}{N} \sum_{i} \frac{1}{q_{i}} \sum_{j \in \Gamma_{i}} \sum_{\left\{\sigma_{l}\right\}} \sum_{\sigma^{\prime}} P\left(“ \vec{\sigma} ", j: \sigma^{\prime}\right) p_{\sigma^{\prime} \rightarrow \sigma_{i}} \sum_{\sigma_{j}} \delta_{\sigma_{s}, \sigma} \delta_{\sigma_{i}, \sigma_{j}} .
$$

A equação 3.3.7 pode ser facilmente adaptada para chegarmos em

$$
D=\frac{1}{N} \sum_{i} \frac{1}{q_{i}} \sum_{j \in \Gamma_{i}} \sum_{\substack{\left\{\sigma_{l}\right\} \\ l \neq j}} \sum_{\sigma^{\prime}} P\left(“ \vec{\sigma} ", j: \sigma^{\prime}\right) p_{\sigma^{\prime} \rightarrow \sigma_{i}}\left(\delta_{j=s} \delta_{\sigma_{i}, \sigma}+\delta_{j \neq s} \delta_{\sigma_{s}, \sigma}\right) .
$$

Fazendo a mudança de variável $\sigma^{\prime}=\sigma_{j}$ temos

$$
D=\frac{1}{N} \sum_{i} \frac{1}{q_{i}} \sum_{j \in \Gamma_{i}} \sum_{\{\vec{\sigma}\}} P(\vec{\sigma}) p_{\sigma_{j} \rightarrow \sigma_{i}}\left(\delta_{j=s} \delta_{\sigma_{i}, \sigma}+\delta_{j \neq s} \delta_{\sigma_{s}, \sigma}\right)
$$


e juntando com $C$ temos

$$
\Delta P(s: \sigma)=\frac{1}{N} \sum_{i} \frac{1}{q_{i}} \sum_{j \in \Gamma_{i}} \delta_{j, s} \sum_{\{\vec{\sigma}\}} P(\vec{\sigma}) p_{\sigma_{j} \rightarrow \sigma_{i}}\left(\delta_{\sigma_{i}, \sigma}-\delta_{\sigma_{s}, \sigma}\right) .
$$

Esses passos seguem essencialmente o que foi feito para o modelo do votante, porém aqui a conta tem um complicador, que não aparece no modelo do votante. Para usar o $\delta_{j, s}$ para eliminar uma das somas, é necessário fazer um reoordenamento. Isso acontece porque o sítio que é modificado não é o primeiro sítio sorteado (no caso do modelo Sznajd esse reoordenamento é ainda mais complicado, pois vai existir uma soma a mais que no caso do Ochrombel). Para entender como deve ser esse reoordenamento, considere

$$
\begin{gathered}
\frac{1}{N} \sum_{i} \frac{1}{q_{i}} \sum_{j \in \Gamma_{i}} \delta_{j, s} \Theta_{i, j, s}=\sum_{i, j} \frac{\delta_{j, s} \Theta_{i, j, s} \delta_{j \in \Gamma_{i}}}{N q_{i}}=\sum_{i} \frac{\Theta_{i, s, s} \delta_{s \in \Gamma_{i}}}{N q_{i}}= \\
=\sum_{i} \frac{\Theta_{i, s, s} \delta_{i \in \Gamma_{s}}}{N q_{i}}=\frac{1}{N} \sum_{i \in \Gamma_{s}} \frac{\Theta_{i, s, s}}{q_{i}}
\end{gathered}
$$

logo a equação 3.4 .7 pode ser reescrita como

$$
\begin{gathered}
\Delta P(s: \sigma)=\frac{1}{N} \sum_{i \in \Gamma_{s}} \frac{1}{q_{i}} \sum_{\{\vec{\sigma}\}} P(\vec{\sigma}) p_{\sigma_{s} \rightarrow \sigma_{i}}\left(\delta_{\sigma_{i}, \sigma}-\delta_{\sigma_{s}, \sigma}\right)= \\
=\frac{1}{N} \sum_{i \in \Gamma_{s}} \frac{1}{q_{i}} \sum_{\sigma^{\prime}}\left(P\left(s: \sigma^{\prime}, i: \sigma\right) p_{\sigma^{\prime} \rightarrow \sigma}-P\left(s: \sigma, i: \sigma^{\prime}\right) p_{\sigma \rightarrow \sigma^{\prime}}\right),
\end{gathered}
$$

que é apenas ligeiramente diferente que a equação para o modelo do votante. Isso era esperado, já que, se a geometria é tal que todos os sítios tem a mesma coordenação, então os 2 modelos são equivalentes, e logo as 2 equações mestras são iguais:

$$
\begin{gathered}
P_{\tau+1}^{(v o t)}(\vec{\sigma})=\frac{1}{N q} \sum_{i} \sum_{j \in \Gamma_{i}}\left(\left(1-p_{\sigma_{i} \rightarrow \sigma_{j}}\right) P_{\tau}(\vec{\sigma})+\sum_{\sigma^{\prime}} P_{\tau}\left(“ \vec{\sigma} ", i: \sigma^{\prime}\right) p_{\sigma^{\prime} \rightarrow \sigma_{j}} \delta_{\sigma_{i}, \sigma_{j}}\right)= \\
=\frac{1}{N q} \sum_{i} \sum_{j \in \Gamma_{i}}(\ldots)=\sum_{i, j} \frac{\delta_{j \in \Gamma_{i}}}{N q}(\ldots)=\sum_{i, j} \frac{\delta_{i \in \Gamma_{j}}}{N q}(\ldots)=\frac{1}{N q} \sum_{j} \sum_{i \in \Gamma_{j}}(\ldots)= \\
=\frac{1}{N q} \sum_{j} \sum_{i \in \Gamma_{j}}\left(\left(1-p_{\sigma_{i} \rightarrow \sigma_{j}}\right) P_{\tau}(\vec{\sigma})+\sum_{\sigma^{\prime}} P_{\tau}\left(“ \vec{\sigma} », i: \sigma^{\prime}\right) p_{\sigma^{\prime} \rightarrow \sigma_{j}} \delta_{\sigma_{i}, \sigma_{j}}\right)= \\
=\frac{1}{N q} \sum_{i} \sum_{j \in \Gamma_{i}}\left(\left(1-p_{\sigma_{j} \rightarrow \sigma_{i}}\right) P_{\tau}(\vec{\sigma})+\sum_{\sigma^{\prime}} P_{\tau}\left(“ \vec{\sigma} ", j: \sigma^{\prime}\right) p_{\sigma^{\prime} \rightarrow \sigma_{i}} \delta_{\sigma_{i}, \sigma_{j}}\right)=P_{\tau+1}^{(o c h)}(\vec{\sigma}) .
\end{gathered}
$$


O análogo da equação 3.3.11 é

$$
\Delta P(s: \sigma)=\frac{1}{N} \sum_{j \in \Gamma_{s}} \frac{1}{q_{j}}(P(j: \sigma)-P(s: \sigma))
$$

e a equação de campo médio que obtemos fazendo as mesmas aproximações é a mesma que para o modelo do votante.

\subsection{O modelo Sznajd}

A equação mestra é dada por

$$
\begin{array}{r}
P_{\tau+1}(\vec{\sigma})=\frac{1}{N} \sum_{i} \frac{1}{q_{i}} \sum_{j \in \Gamma_{i}}(\underbrace{\left(1-\delta_{\sigma_{i}, \sigma_{j}}\right) P_{\tau}(\vec{\sigma})}_{A}+\delta_{\sigma_{i}, \sigma_{j}} \frac{1}{q_{j}}(\underbrace{P_{\tau}(\vec{\sigma})}_{B}+ \\
\left.\left.+\sum_{k \in \Gamma_{j}-\{i\}}(\underbrace{\left(1-p_{\sigma_{k} \rightarrow \sigma_{i}}\right) P_{\tau}(\vec{\sigma})}_{C}+\sum_{\sigma^{\prime}} \underbrace{P_{\tau}\left(“ \vec{\sigma} ", k: \sigma^{\prime}\right) p_{\sigma^{\prime} \rightarrow \sigma_{i}} \delta_{\sigma_{k}, \sigma_{i}}}_{D})\right)\right) .
\end{array}
$$

Em cada um dos termos da equação 3.5.1, $i$ indica o primeiro sítio que foi sorteado nessa iteração, $j$ indica o vizinho dele e $k$ o vizinho de $j$, que $i$ e $j$ estão tentando convencer. Os termos $A, B$ e $C$ correspondem a casos em que o estado não muda (logo, eles devem ser ponderados por $P_{\tau}(\vec{\sigma})$ ), sendo que os termos $A$ e $B$ se referem a situações novas que ocorrem no modelo Sznajd. $A$ contabiliza os casos em que $i$ e $j$ discordam, $B$ contabiliza o caso em que $i$ e $k$ são o mesmo sítio e $C$ é o caso em que $i$ e $j$ concordam, mas não conseguem convencer $k$ por causa da regra de confiança (esse processo já aparecia nos modelos do votante e Ochrombel). O termo $D$ é o responsável pelos convencimentos bem sucedidos, mais especificamente, ele contabiliza o caso em que $k$ tinha opinião $\sigma^{\prime}$ antes do convencimento, logo ele é ponderado por $P_{\tau}$ ( " $\vec{\sigma}$ ", $\left.k: \sigma^{\prime}\right)$ e deve ser proporcional a $\delta_{\sigma_{k}, \sigma_{i}}$ para filtrar os casos em que o estado $\vec{\sigma}$ não é compatível com uma interação bem sucedida entre $i, j$ e $k$. Assim como fizemos com o modelo do votante e o modelo Ochrombel, vamos transformar a equação em uma equação de diferenças e depois somar sobre $\vec{\sigma}$ ponderando por $\delta_{\sigma_{s}, \sigma}$ para marginalizar a distribuição. Depois vamos nos focar no termo que vem de $D$, separando a soma em $\sigma_{k}$, para depois fazer a mudança de variável $\sigma^{\prime}=\sigma_{k}$, reescrevendo o termo em função de $P(\vec{\sigma})$ e reestabelecendo a soma em $\vec{\sigma}$.

Lembrando da equação 3.3.2, podemos reescrever 3.5.1 como

$$
\begin{gathered}
\Delta P(\vec{\sigma})=\frac{1}{N} \sum_{i} \frac{1}{q_{i}} \sum_{j \in \Gamma_{i}} \delta_{\sigma_{i}, \sigma_{j}}\left(-P(\vec{\sigma})+\frac{1}{q_{j}}(P(\vec{\sigma})+\right. \\
\left.\left.+\sum_{k \in \Gamma_{j}-\{i\}}\left(\left(1-p_{\sigma_{k} \rightarrow \sigma_{i}}\right) P(\vec{\sigma})+\sum_{\sigma^{\prime}} P\left(“ \vec{\sigma} ", k: \sigma^{\prime}\right) p_{\sigma^{\prime} \rightarrow \sigma_{i}} \delta_{\sigma_{k}, \sigma_{i}}\right)\right)\right),
\end{gathered}
$$


Os termos $A, B$ e $C$ podem ser combinados para simplificar essa equação:

$$
\begin{gathered}
\Delta P(\vec{\sigma})=\frac{1}{N} \sum_{i} \frac{1}{q_{i}} \sum_{j \in \Gamma_{i}} \delta_{\sigma_{i}, \sigma_{j}}\left(-P(\vec{\sigma})+\frac{1}{q_{j}}(P(\vec{\sigma})+\right. \\
\left.\left.+\sum_{k \in \Gamma_{j}-\{i\}}\left(\left(1-p_{\sigma_{k} \rightarrow \sigma_{i}}\right) P(\vec{\sigma})+\sum_{\sigma^{\prime}} P\left(“ \vec{\sigma} ", k: \sigma^{\prime}\right) p_{\sigma^{\prime} \rightarrow \sigma_{i}} \delta_{\sigma_{k}, \sigma_{i}}\right)\right)\right),
\end{gathered}
$$

resultando em

$$
\Delta P(\vec{\sigma})=\frac{1}{N} \sum_{i} \frac{1}{q_{i}} \sum_{j \in \Gamma_{i}} \frac{\delta_{\sigma_{i}, \sigma_{j}}}{q_{j}} \sum_{k \in \Gamma_{j}-\{i\}}(\underbrace{-p_{\sigma_{k} \rightarrow \sigma_{i}} P(\vec{\sigma})}_{E}+\underbrace{\sum_{\sigma^{\prime}} P\left(" \vec{\sigma} ", k: \sigma^{\prime}\right) p_{\sigma^{\prime} \rightarrow \sigma_{i}} \delta_{\sigma_{k}, \sigma_{i}}}_{F}),
$$

que é bem semelhante às equações obtidas para os outros modelos (apenas com uma soma extra adicionada). Dessa forma, podemos interpretar o termo $E$ como contabilizando as situações em que já estamos no estado $\vec{\sigma}$ e um convencimento bem sucedido nos leva a um estado diferente (note que quando $\sigma_{i}=\sigma_{k}$ esse termo não contribui pois $p_{\sigma_{k} \rightarrow \sigma_{i}}=0$ nesse caso), diminuindo $P(\vec{\sigma})$. O termo $F$ pode ser interpretado como contabilizando todas as formas de um estado chegar em $\vec{\sigma}$ a partir de um convencimento bem sucedido, logo aumentando $P(\vec{\sigma})$. Marginalizando a distribuição (novamente usando a equação 3.3.4) temos

$$
\begin{gathered}
\Delta P(s: \sigma)=\frac{1}{N} \sum_{i} \frac{1}{q_{i}} \sum_{j \in \Gamma_{i}} \frac{1}{q_{j}} \sum_{k \in \Gamma_{j}-\{i\}} \sum_{\{\vec{\sigma}\}} \delta_{\sigma_{s}, \sigma} \delta_{\sigma_{i}, \sigma_{j}}\left(-p_{\sigma_{k} \rightarrow \sigma_{i}} P(\vec{\sigma})+\right. \\
\left.\sum_{\sigma^{\prime}} P\left(“ \vec{\sigma} ", k: \sigma^{\prime}\right) p_{\sigma^{\prime} \rightarrow \sigma_{i}} \delta_{\sigma_{k}, \sigma_{i}}\right) .
\end{gathered}
$$

O termo proveniente de $D$ (e de $F$ ) fica

$$
\sum_{\{\vec{\sigma}\}} \delta_{\sigma_{s}, \sigma} \delta_{\sigma_{i}, \sigma_{j}} \sum_{\sigma^{\prime}} P\left(“ \vec{\sigma} ", k: \sigma^{\prime}\right) p_{\sigma^{\prime} \rightarrow \sigma_{i}} \delta_{\sigma_{k}, \sigma_{i}} .
$$

Vamos reoordenar as somas, separando a soma em $\sigma_{k}$ e chegando em

$$
\sum_{\substack{\left\{\sigma_{l}\right\} \\ l \neq k}} \sum_{\sigma^{\prime}} \delta_{\sigma_{i}, \sigma_{j}} P\left(" \vec{\sigma} ", k: \sigma^{\prime}\right) p_{\sigma^{\prime} \rightarrow \sigma_{i}} \sum_{\sigma_{k}} \delta_{\sigma_{k}, \sigma_{i}} \delta_{\sigma_{s}, \sigma},
$$

pois $k \neq i, j$ e $P\left(\right.$ " $\vec{\sigma}$ ", $\left.k: \sigma^{\prime}\right)$ é independente de $\sigma_{k}$. Relembrando a equação 3.3.7, temos

$$
\sum_{\sigma_{k}} \delta_{\sigma_{k}, \sigma_{i}} \delta_{\sigma_{s}, \sigma}=\delta_{s \neq k} \delta_{\sigma_{s}, \sigma}+\delta_{s, k} \delta_{\sigma, \sigma_{i}}
$$


e logo, substituindo em 3.5 .5 vem

$$
\sum_{\substack{\left\{\sigma_{l}\right\} \\ l \neq k}} \sum_{\sigma^{\prime}} \delta_{\sigma_{i}, \sigma_{j}} P\left(“ \vec{\sigma} ", k: \sigma^{\prime}\right) p_{\sigma^{\prime} \rightarrow \sigma_{i}}\left(\delta_{s \neq k} \delta_{\sigma_{s}, \sigma}+\delta_{s, k} \delta_{\sigma, \sigma_{i}}\right) .
$$

Fazemos a mudança de variável $\sigma^{\prime}=\sigma_{k}$ e chegamos finalmente em

$$
\sum_{\{\vec{\sigma}\}} \delta_{\sigma_{i}, \sigma_{j}} P(\vec{\sigma}) p_{\sigma_{k} \rightarrow \sigma_{i}}\left(\delta_{s \neq k} \delta_{\sigma_{s}, \sigma}+\delta_{s, k} \delta_{\sigma, \sigma_{i}}\right) .
$$

Substituindo na equação 3.5.4 e usando $\delta_{s \neq k}+\delta_{s, k}=1$ vem

$$
\begin{gathered}
\Delta P(s: \sigma)=\frac{1}{N} \sum_{i} \frac{1}{q_{i}} \sum_{j \in \Gamma_{i}} \frac{1}{q_{j}} \sum_{k \in \Gamma_{j}-\{i\}} \sum_{\{\vec{\sigma}\}}\left(-P(\vec{\sigma})\left(\delta_{s \neq k}+\delta_{s, k}\right) \delta_{\sigma_{s}, \sigma} \delta_{\sigma_{i}, \sigma_{j}} p_{\sigma_{k} \rightarrow \sigma_{i}}+\right. \\
\left.\quad+P(\vec{\sigma}) p_{\sigma_{k} \rightarrow \sigma_{i}} \delta_{\sigma_{i}, \sigma_{j}}\left(\delta_{(s \neq k)} \delta_{\sigma_{s}, \sigma}+\delta_{s, k} \delta_{\sigma, \sigma_{i}}\right)\right) \Rightarrow \\
\Delta P(s: \sigma)=\frac{1}{N} \sum_{i} \frac{1}{q_{i}} \sum_{j \in \Gamma_{i}} \frac{1}{q_{j}} \sum_{k \in \Gamma_{j}-\{i\}} \delta_{s, k} \sum_{\{\vec{\sigma}\}} P(\vec{\sigma}) p_{\sigma_{s} \rightarrow \sigma_{i}}\left(\delta_{\sigma_{i}, \sigma_{j}, \sigma}-\delta_{\sigma_{s}, \sigma} \delta_{\sigma_{i}, \sigma_{j}}\right) .
\end{gathered}
$$

Iremos agora reordenar as somas em $i, j$ e $k$, para usar o termo $\delta_{s, k}$. Para isso, considere a soma

$$
\begin{gathered}
\frac{1}{N} \sum_{i} \frac{1}{q_{i}} \sum_{j \in \Gamma_{i}} \frac{1}{q_{j}} \sum_{k \in \Gamma_{j}-\{i\}} \delta_{s, k} \Theta_{i, j, k, s}=\sum_{i, j, k} \frac{\delta_{j \in \Gamma_{i}} \delta_{k \in \Gamma_{j}} \delta_{k \neq i} \delta_{s, k} \Theta_{i, j, k, s}}{N q_{i} q_{j}}= \\
=\sum_{i, j} \frac{\delta_{j \in \Gamma_{i}} \delta_{s \in \Gamma_{j}} \delta_{s \neq i} \Theta_{i, j, s, s}}{N q_{i} q_{j}} .
\end{gathered}
$$

Como $j \in \Gamma_{i} \Leftrightarrow i \in \Gamma_{j}$ e $s \in \Gamma_{j} \Leftrightarrow j \in \Gamma_{s}$, temos

$$
\sum_{i, j} \frac{\delta_{i \in \Gamma_{j}} \delta_{j \in \Gamma_{s}} \delta_{s \neq i} \Theta_{i, j, s, s}}{N q_{i} q_{j}}=\frac{1}{N} \sum_{j \in \Gamma_{s}} \frac{1}{q_{j}} \sum_{i \in \Gamma_{j}} \frac{\delta_{s \neq i} \Theta_{i, j, s, s}}{q_{i}}=\frac{1}{N} \sum_{j \in \Gamma_{s}} \frac{1}{q_{j}} \sum_{i \in \Gamma_{j}-\{s\}} \frac{\Theta_{i, j, s, s}}{q_{i}} .
$$

Aplicando na equação 3.5 .8 chegamos em

$$
\Delta P(s: \sigma)=\frac{1}{N} \sum_{j \in \Gamma_{s}} \frac{1}{q_{j}} \sum_{i \in \Gamma_{j}-\{s\}} \frac{1}{q_{i}} \sum_{\{\vec{\sigma}\}} P(\vec{\sigma}) p_{\sigma_{s} \rightarrow \sigma_{i}}\left(\delta_{\sigma_{i}, \sigma_{j}, \sigma}-\delta_{\sigma_{s}, \sigma} \delta_{\sigma_{i}, \sigma_{j}}\right) .
$$

Finalmente, fazemos a soma em $\vec{\sigma}$ e chegamos no análogo das equações 3.3 .10 e 3.4 .8 para o modelo Sznajd 


$$
\begin{aligned}
& \Delta P(s: \sigma)=\frac{1}{N} \sum_{j \in \Gamma_{s}} \frac{1}{q_{j}} \sum_{i \in \Gamma_{j}-\{s\}} \frac{1}{q_{i}} \sum_{\sigma_{i}, \sigma_{j}, \sigma_{s}} P\left(i: \sigma_{i}, j: \sigma_{j}, s: \sigma_{s}\right) p_{\sigma_{s} \rightarrow \sigma_{i}}\left(\delta_{\sigma_{i}, \sigma_{j}, \sigma}-\delta_{\sigma_{s}, \sigma} \delta_{\sigma_{i}, \sigma_{j}}\right)= \\
& =\frac{1}{N} \sum_{j \in \Gamma_{s}} \frac{1}{q_{j}} \sum_{i \in \Gamma_{j}-\{s\}} \frac{1}{q_{i}}\left(\sum_{\sigma_{s}} P\left(i: \sigma, j: \sigma, s: \sigma_{s}\right) p_{\sigma_{s} \rightarrow \sigma}-\sum_{\sigma_{i}} P\left(i: \sigma_{i}, j: \sigma_{i}, s: \sigma\right) p_{\sigma \rightarrow \sigma_{i}}\right) \Rightarrow \\
& \Delta P(s: \sigma)=\frac{1}{N} \sum_{j \in \Gamma_{s}} \frac{1}{q_{j}} \sum_{i \in \Gamma_{j}-\{s\}} \frac{1}{q_{i}} \sum_{\sigma^{\prime}}\left(P\left(i: \sigma, j: \sigma, s: \sigma^{\prime}\right) p_{\sigma^{\prime} \rightarrow \sigma}-P\left(i: \sigma^{\prime}, j: \sigma^{\prime}, s: \sigma\right) p_{\sigma \rightarrow \sigma^{\prime}}\right) .
\end{aligned}
$$

Note que o termo $\left(P\left(i: \sigma, j: \sigma, s: \sigma^{\prime}\right) p_{\sigma^{\prime} \rightarrow \sigma}-P\left(i: \sigma^{\prime}, j: \sigma^{\prime}, s: \sigma\right) p_{\sigma \rightarrow \sigma^{\prime}}\right)$ seria 0 no caso $i=s$, logo a restrição $i \in \Gamma_{j}-\{s\}$ (na segunda soma) é equivalente a $i \in \Gamma_{j}$. Também é importante notar que em uma geometria geral isso dá um sistema de equações que não fecham mesmo se não usarmos regras de confiança (ao contrário do acontece para os modelos do votante e Ochrombel). Fazendo uma aproximação parecida com a que fizemos para o modelo do votante chegamos em

$$
\begin{gathered}
\Delta P(s: \sigma)=\frac{1}{N} \sum_{j \in \Gamma_{s}} \frac{1}{q_{j}} \sum_{i \in \Gamma_{j}} \frac{1}{q_{i}} \sum_{\sigma^{\prime}}\left(P(i: \sigma) P(j: \sigma) P\left(s: \sigma^{\prime}\right) p_{\sigma^{\prime} \rightarrow \sigma}+\right. \\
\left.-P\left(i: \sigma^{\prime}\right) P\left(j: \sigma^{\prime}\right) P(s: \sigma) p_{\sigma \rightarrow \sigma^{\prime}}\right) .
\end{gathered}
$$

Colocando o modelo em um grafo completo vem

$$
\begin{gathered}
\Delta P(s: \sigma)=\frac{1}{N(N-1)^{2}} \sum_{j \neq s} \sum_{i \neq j} \sum_{\sigma^{\prime}}\left(P(i: \sigma) P(j: \sigma) P\left(s: \sigma^{\prime}\right) p_{\sigma^{\prime} \rightarrow \sigma}+\right. \\
\left.-P\left(i: \sigma^{\prime}\right) P\left(j: \sigma^{\prime}\right) P(s: \sigma) p_{\sigma \rightarrow \sigma^{\prime}}\right)= \\
\frac{1}{N^{3}} \sum_{i, j} \sum_{\sigma^{\prime}}\left(P(i: \sigma) P(j: \sigma) P\left(s: \sigma^{\prime}\right) p_{\sigma^{\prime} \rightarrow \sigma}-P\left(i: \sigma^{\prime}\right) P\left(j: \sigma^{\prime}\right) P(s: \sigma) p_{\sigma \rightarrow \sigma^{\prime}}\right)+\mathcal{O}\left(\frac{1}{N^{2}}\right) \Rightarrow \\
\Delta \eta_{\sigma}=\frac{1}{N^{4}} \sum_{i, j, s} \sum_{\sigma^{\prime}}\left(P(i: \sigma) P(j: \sigma) P\left(s: \sigma^{\prime}\right) p_{\sigma^{\prime} \rightarrow \sigma}+\right. \\
\left.-P\left(i: \sigma^{\prime}\right) P\left(j: \sigma^{\prime}\right) P(s: \sigma) p_{\sigma \rightarrow \sigma^{\prime}}\right)+\mathcal{O}\left(\frac{1}{N^{2}}\right)= \\
=\frac{1}{N} \sum_{\sigma^{\prime}}\left(\eta_{\sigma}^{2} \eta_{\sigma^{\prime}} p_{\sigma^{\prime} \rightarrow \sigma}-\eta_{\sigma} \eta_{\sigma^{\prime}}^{2} p_{\sigma \rightarrow \sigma^{\prime}}\right)+\mathcal{O}\left(\frac{1}{N^{2}}\right) .
\end{gathered}
$$

No limite termodinâmico isso é equivalente a integrar o fluxo 


$$
\dot{\eta}_{\sigma}=\sum_{\sigma^{\prime}} \eta_{\sigma} \eta_{\sigma^{\prime}}\left(\eta_{\sigma} p_{\sigma^{\prime} \rightarrow \sigma}-\eta_{\sigma^{\prime}} p_{\sigma \rightarrow \sigma^{\prime}}\right),
$$

que é a equação de campo médio que usamos para o modelo Sznajd.

\subsection{Observações sobre essas deduções}

Como a equação de diferenças que gera a equação de campo médio é um mapa, em que cada passo de tempo equivale a uma iteração de um método de Euler, integrando a equação de campo médio com passo $1 / N$, então a unidade de tempo das equações de campo médio é o tempo de Monte Carlo, ou seja, o número de iterações para que um sítio seja visitado uma vez em média na rede. Como $\eta_{\sigma}$ é definido como sendo o valor esperado para a proporção de sítios da rede que tem opinião $\sigma$, então, para fazermos comparações entre as equações de campo médio e as simulações, devemos nos lembrar que as equações de campo médio lidam com valores esperados. Isso implica que as condições iniciais do fluxo são as probabilidades com que as opiniões são sorteadas na rede (no caso em que todos os sítios são sorteados usando as mesmas probabilidades) e as trajetórias do fluxo devem ser comparadas com médias sobre realizações na rede.

Para chegar nas equações de campo médio, nós precisamos assumir que as distribuições marginais de 2 (para o caso do votante e do modelo Ochrombel) e de 3 (para o modelo Sznajd) sítios eram dadas pelo produto das distribuições marginais de 1 sítio. Em outras palavras, estamos assumindo que a probabilidade de um sítio ter uma dada opinião é independente da probabilidade de os seus vizinhos terem outra opinião dada. Se as opiniões forem sorteadas de maneira aleatória, então isso deve ser verdadeiro para tempos curtos. Porém, a medida que o tempo passa, vão se formando correlações entre os sítios e essa aproximação pode deixar de ser verdade. Por outro lado, se fizermos simulações em uma rede completa, as correlações são irrelevantes e a aproximação vale sempre, ou seja, a geometria também deve ter um efeito na qualidade dessa aproximação. 



\title{
Capítulo 4
}

\section{Resultados Analíticos para a aproximação de campo médio}

\author{
"I understand what an equation means if I have a way of figuring out the \\ characteristics of its solution without actually solving it."
}

- Paul Dirac

Iremos nesse capítulo apresentar a aproximação de campo médio (que foi deduzida no capítulo 3) e estabelecer algumas propriedades das trajetórias, para depois estudar o que ocorre com a introdução de ruído, com o modelo do votante e com generalizações do modelo Sznajd. Primeiramente nós iremos estabelecer algumas propriedades básicas dessas equações, como estabelecer quais estados fazem sentido no contexto do nosso modelo e mostrar que a evolução temporal é consistente no sentido de não gerar probabilidades negativas e das probabilidades sempre somarem 1. Depois disso, nós iremos fazer o estudo de como as trajetórias se comportam na vizinhança de pontos fixos. Nessa parte, ao invés de calcular os pontos fixos em função dos parâmetros da regra de confiança (o que é completamente impraticável, já que esses pontos fixos seriam soluções de sistemas de equações algébricas não-lineares e que são a priori arbitrariamente grandes), nós assumimos que um ponto fixo com determinadas propriedades exista e vemos as conclusões a que essa hipótese nos leva, posteriormente usando teoremas de topologia para determinar quando esses pontos hipotéticos existem e quando eles não existem. Ao final de nossa análise chegamos a importante conclusão de que todas as propriedades de estabilidade de um ponto fixo dependem apenas de quais são as opiniões que sobrevivem nele e de qual é o esqueleto direcionado da regra de confiança que está sendo usada e descobrimos também que os pontos fixos que uma determinada regra exibe também são inteiramente determinados pelo esqueleto direcionado dessa regra. Além disso, tanto as propriedades de estabilidade quanto de existência de pontos fixos podem ser expressas em termos de problemas de teoria de grafos (mais precisamente do problema de conjuntos independentes maximais e do problema de componentes fortemente conectadas). A seguir, resolvemos o modelo usual com ruído (no sentido de determinar os 
seus pontos fixos e as propriedades de estabilidade), determinando todas as bifurcações que ocorrem a medida que a intensidade do ruído vai aumentando. Depois, fazemos um tratamento perturbativo do modelo com regras de confiança e ruído infinitesimal, determinando quais das estruturas que existem no modelo sem ruído não sobrevivem à adição desse ruído infinitesimal e o que ocorre com as que sobrevivem. Além disso, com a adição desse ruído, passamos a ter a possibilidade do surgimento de novas estruturas como ciclos limite e atratores estranhos. Na sequência, analisamos uma generalização do modelo Sznajd com regras de confiança em que é necessário formar um grupo de tamanho $q$ para que ocorra convencimento e mostramos que os resultados são exatamente os mesmos de um ponto de vista qualitativo. Finalmente, fazemos o tratamento do modelo do votante, mostrando quais são as diferenças entre ele e o modelo Sznajd na aproximação de campo médio.

\subsection{Considerações iniciais}

Como vimos no capítulo 3, a equação de campo médio do modelo Sznajd é dada por

$$
\dot{\eta}_{\sigma}=F_{\sigma}=\sum_{\sigma^{\prime}} \eta_{\sigma} \eta_{\sigma^{\prime}}\left(\eta_{\sigma} p_{\sigma^{\prime} \rightarrow \sigma}-\eta_{\sigma^{\prime}} p_{\sigma \rightarrow \sigma^{\prime}}\right)
$$

Suponha que estejamos analisando um modelo com $M$ opiniões. Então essa equação tem $M$ variáveis dadas pelos $\eta_{\sigma}$, que são a proporção de sítios na rede com opinião $\sigma$, ou de forma equivalente, a probabilidade que um sítio escolhido ao acaso tenha opinião $\sigma$. Como todos os sítios tem alguma opinião, segue que os estados que fazem sentido são dados pelo simplexo

$$
\operatorname{Sim}_{M}=\left\{\vec{\eta} \in \mathbb{R}^{M} \mid \eta_{\sigma} \geq 0 \text { e } \sum_{\sigma} \eta_{\sigma}=1\right\} \text {. }
$$

Primeiramente devemos mostrar que as equações 4.1.1 são consistentes. Para tanto, precisamos mostrar que uma trajetória que começa nesse simplexo (que será o nosso espaço de fase) nunca o deixa (quando tratamos a equação como sendo um fluxo em $\mathbb{R}^{M}$ ), ou seja, devemos mostrar que, se todas as coordenadas começam positivas e somando 1, elas permanecem com essa propriedade ao longo de toda a trajetória (o caso em que alguma das coordenadas começa igual a 0 não precisa ser analisado, pois a derivada dessa coordenada seria sempre $0)$. Das equações 4.1.1 vem que

$$
\frac{\mathrm{d}}{\mathrm{dt}} \ln \left|\eta_{\sigma}\right|=\frac{\dot{\eta}_{\sigma}}{\eta_{\sigma}}=\sum_{\sigma^{\prime}} \eta_{\sigma^{\prime}}\left(\eta_{\sigma} p_{\sigma^{\prime} \rightarrow \sigma}-\eta_{\sigma^{\prime}} p_{\sigma \rightarrow \sigma^{\prime}}\right) .
$$

Logo, se uma trajetória começa no interior de $\operatorname{Sim}_{M}$, ela é tal que 


$$
\left|\frac{\mathrm{d}}{\mathrm{dt}} \ln \right| \eta_{\sigma}||=\left|\sum_{\sigma^{\prime}} \eta_{\sigma^{\prime}}\left(\eta_{\sigma} p_{\sigma^{\prime} \rightarrow \sigma}-\eta_{\sigma^{\prime}} p_{\sigma \rightarrow \sigma^{\prime}}\right)\right| \leq \sum_{\sigma^{\prime}} \eta_{\sigma^{\prime}}\left(\left|\eta_{\sigma} p_{\sigma^{\prime} \rightarrow \sigma}\right|+\left|\eta_{\sigma^{\prime}} p_{\sigma \rightarrow \sigma^{\prime}}\right|\right) \leq 2 M
$$

Isso implica que nenhuma variável se torna negativa, pois como as trajetórias são contínuas seria necessário que uma das variáveis se anulasse em um tempo finito, o que implicaria que para algum $\sigma$, a grandeza $\ln \left|\eta_{\sigma}\right|$ deveria divergir para $-\infty$ em um tempo finito, o que é proibido pelas desigualdades em 4.1.2, uma vez que elas implicam que

$$
\ln \left|\eta_{\sigma}\right| \geq \ln \left|\eta_{\sigma o}\right|-2 M t \forall \sigma .
$$

Falta então apenas mostrar que a soma das variáveis permanece igual. Somando todas as equações em 4.1.1 vem

$$
\frac{\mathrm{d}}{\mathrm{dt}} \sum_{\sigma} \eta_{\sigma}=\sum_{\sigma} \dot{\eta}_{\sigma}=\sum_{\sigma, \sigma^{\prime}} \eta_{\sigma} \eta_{\sigma^{\prime}}\left(\eta_{\sigma} p_{\sigma^{\prime} \rightarrow \sigma}-\eta_{\sigma^{\prime}} p_{\sigma \rightarrow \sigma^{\prime}}\right)=0,
$$

pois $\eta_{\sigma} \eta_{\sigma^{\prime}}\left(\eta_{\sigma} p_{\sigma^{\prime} \rightarrow \sigma}-\eta_{\sigma^{\prime}} p_{\sigma \rightarrow \sigma^{\prime}}\right)$ é antissimétrico por uma troca de $\sigma$ por $\sigma^{\prime}$.

\subsection{Pontos fixos e estabilidade}

Uma vez estabelecida a consistência das equações de campo médio, iremos analisar o comportamento das trajetórias na vizinhança dos pontos fixos. Iremos apresentar a equação de pontos fixos e escrever o jacobiano de $\vec{F}$. A principal ferramenta que iremos utilizar é a análise de estabilidade linear, ou seja, avaliar o jacobiano no ponto fixo e encontrar os autovalores dele. Lembramos que se a parte real de todos esses autovalores for diferente de 0 essa análise dá a informação completa do comportamento das trajetórias em torno desses pontos (ver a seção 1.3, ou o teorema A.4.2 para uma apresentação mais precisa).

Os pontos fixos da eq. 4.1.1 são os pontos de $\operatorname{Sim}_{M}$ que satisfazem o seguinte sistema de equações

$$
\eta_{\sigma}=0 \quad \text { ou } \quad \sum_{\sigma^{\prime}} \eta_{\sigma^{\prime}}\left(\eta_{\sigma} p_{\sigma^{\prime} \rightarrow \sigma}-\eta_{\sigma^{\prime}} p_{\sigma \rightarrow \sigma^{\prime}}\right)=0 \quad \forall \sigma
$$

Toda regra de confiança tem pelo menos $M$ pontos fixos triviais, pois se $\eta_{\sigma} \eta_{\sigma^{\prime}}=0 \forall \sigma \neq \sigma^{\prime}$, temos uma solução de 4.2.1. Esses pontos fixos correspondem aos estados de consenso, em que todos os sítios tem a mesma opinião.

De forma mais geral, para cada ponto fixo $\vec{\eta}^{*}$ podemos definir $\Omega$ como sendo o conjunto de opiniões tais que $\eta_{\sigma}^{*}=0$ (logo as opiniões que foram "extintas") e $\Delta=\bar{\Omega}$ (as opiniões "sobreviventes"). Para fazer a análise de estabilidade linear precisamos calcular o jacobiano de $\vec{F}$ (como definido na eq. 4.1.1), $\mathcal{J}_{\vec{F}}$ : 


$$
\begin{gathered}
\left(\mathcal{J}_{\vec{F}}\right)_{\sigma, \sigma^{\prime}}=\frac{\partial F_{\sigma}}{\partial \eta_{\sigma^{\prime}}}=\frac{\partial}{\partial \eta_{\sigma^{\prime}}} \sum_{\sigma^{\prime \prime}} \eta_{\sigma} \eta_{\sigma^{\prime \prime}}\left(\eta_{\sigma} p_{\sigma^{\prime \prime} \rightarrow \sigma}-\eta_{\sigma^{\prime \prime}} p_{\sigma \rightarrow \sigma^{\prime \prime}}\right)= \\
=\sum_{\sigma^{\prime \prime}}\left(\left(\frac{\partial \eta_{\sigma}}{\partial \eta_{\sigma^{\prime}}} \eta_{\sigma^{\prime \prime}}+\eta_{\sigma} \frac{\partial \eta_{\sigma^{\prime \prime}}}{\partial \eta_{\sigma^{\prime}}}\right)\left(\eta_{\sigma} p_{\sigma^{\prime \prime} \rightarrow \sigma}-\eta_{\sigma^{\prime \prime}} p_{\sigma \rightarrow \sigma^{\prime \prime}}\right)+\eta_{\sigma} \eta_{\sigma^{\prime \prime}}\left(\frac{\partial \eta_{\sigma}}{\partial \eta_{\sigma^{\prime}}} p_{\sigma^{\prime \prime} \rightarrow \sigma}-\frac{\partial \eta_{\sigma^{\prime \prime}}}{\partial \eta_{\sigma^{\prime}}} p_{\sigma \rightarrow \sigma^{\prime \prime}}\right)\right)= \\
=\sum_{\sigma^{\prime \prime}}\left(\left(\delta_{\sigma, \sigma^{\prime}} \eta_{\sigma^{\prime \prime}}+\eta_{\sigma} \delta_{\sigma^{\prime}, \sigma^{\prime \prime}}\right)\left(\eta_{\sigma} p_{\sigma^{\prime \prime} \rightarrow \sigma}-\eta_{\sigma^{\prime \prime}} p_{\sigma \rightarrow \sigma^{\prime \prime}}\right)+\eta_{\sigma} \eta_{\sigma^{\prime \prime}}\left(\delta_{\sigma, \sigma^{\prime}} p_{\sigma^{\prime \prime} \rightarrow \sigma}-\delta_{\sigma^{\prime}, \sigma^{\prime \prime}} p_{\sigma \rightarrow \sigma^{\prime \prime}}\right)\right) \Rightarrow \\
\left(\mathcal{J}_{\vec{F}}\right)_{\sigma, \sigma^{\prime}}=\delta_{\sigma, \sigma^{\prime}} \sum_{\sigma^{\prime \prime}}\left(2 \eta_{\sigma} \eta_{\sigma^{\prime \prime}} p_{\sigma^{\prime \prime} \rightarrow \sigma}-\eta_{\sigma^{\prime \prime}}^{2} p_{\sigma \rightarrow \sigma^{\prime \prime}}\right)+\eta_{\sigma}^{2} p_{\sigma^{\prime} \rightarrow \sigma}-2 \eta_{\sigma} \eta_{\sigma^{\prime}} p_{\sigma \rightarrow \sigma^{\prime}} .
\end{gathered}
$$

Ao invés de encontrar os pontos fixos da equação 4.1.1, o que envolveria resolver um sistema cúbico de $M$ equações e $M$ incógnitas, iremos assumir que exista uma solução $\vec{\eta}^{*} \in$ $\operatorname{Sim}_{M}$ para as equações de campo médio. Num primeiro momento iremos apenas escrever os resultados em termos das coordenadas dessa solução hipotética, sem nos preocuparmos se ela existe ou não, e a única propriedade que iremos impor sobre as coordenadas de $\vec{\eta}^{*}$ é que elas satisfazem as equações de campo médio. Seja $\mathcal{J}^{*}$ o jacobiano avaliado em $\vec{\eta}^{*}$. Nesse caso temos

$$
\mathcal{J}_{\sigma, \sigma^{\prime}}^{*}= \begin{cases}-\delta_{\sigma, \sigma^{\prime}} \sum_{\sigma^{\prime \prime}} \eta_{\sigma^{\prime \prime}}^{* 2} p_{\sigma \rightarrow \sigma^{\prime \prime}}, & \text { se } \sigma \in \Omega \\ \eta_{\sigma}^{* 2} p_{\sigma^{\prime} \rightarrow \sigma}-2 \eta_{\sigma}^{*} \eta_{\sigma^{\prime}}^{*} p_{\sigma \rightarrow \sigma^{\prime}}+\delta_{\sigma, \sigma^{\prime}} \sum_{\sigma^{\prime \prime}} \eta_{\sigma}^{*} \eta_{\sigma^{\prime \prime}}^{*} p_{\sigma^{\prime \prime} \rightarrow \sigma}, & \text { se } \sigma \in \Delta\end{cases}
$$

e portanto sempre existe uma permutação que leva $\mathcal{J}^{*}$ em

$$
\mathcal{J}^{*}=\left[\begin{array}{cc}
\mathcal{J}_{\Delta}^{*} & \mathcal{N} \\
0 & \mathcal{J}_{\Omega}^{*}
\end{array}\right]
$$

Onde $\mathcal{J}_{\Delta}^{*}$ é o jacobiano restrito às opiniões sobreviventes e $\mathcal{J}_{\Omega}^{*}$ é o restrito às opiniões extintas. Como $\mathcal{J}^{*}$ é bloco triangular, a matriz $\mathcal{N}$ não interfere nos autovalores, pois se $\vec{x}$ é tal que $\mathcal{J}_{\Delta}^{*} \cdot \vec{x}=\lambda \vec{x}$, então

$$
\mathcal{J}^{*}=\left[\begin{array}{cc}
\mathcal{J}_{\Delta}^{*} & \mathcal{N} \\
0 & \mathcal{J}_{\Omega}^{*}
\end{array}\right] \cdot\left[\begin{array}{c}
\vec{x} \\
\overrightarrow{0}
\end{array}\right]=\left[\begin{array}{c}
\mathcal{J}_{\Delta}^{*} \cdot \vec{x} \\
\overrightarrow{0}
\end{array}\right]=\lambda\left[\begin{array}{c}
\vec{x} \\
\overrightarrow{0}
\end{array}\right]
$$

e se $\vec{y}$ é tal que $\vec{y} \cdot \mathcal{J}_{\Omega}^{*}=\lambda \vec{y}$, então

$$
\left[\begin{array}{ll}
\overrightarrow{0} & \vec{y}
\end{array}\right] \cdot \mathcal{J}^{*}=\left[\begin{array}{cc}
\mathcal{J}_{\Delta}^{*} & \mathcal{N} \\
0 & \mathcal{J}_{\Omega}^{*}
\end{array}\right]=\left[\begin{array}{ll}
\overrightarrow{0} & \vec{y} \cdot \mathcal{J}_{\Omega}^{*}
\end{array}\right]=\lambda\left[\begin{array}{ll}
\overrightarrow{0} & \vec{y}
\end{array}\right] .
$$

Logo o espectro de $\mathcal{J}^{*}$ é a "união" dos espectros de $\mathcal{J}_{\Delta}^{*}$ e $\mathcal{J}_{\Omega}^{*}$. Essa é uma propriedade importante, pois significa que podemos separar a nossa análise em partes. Além disso, como $\mathcal{J}_{\Omega}^{*}$ é uma matriz diagonal (o termo do jacobiano em 4.2.3 quando $\sigma \in \Omega$ é proporcional a $\left.\delta_{\sigma, \sigma^{\prime}}\right)$, temos os seus autovalores automaticamente, de forma que iremos primeiro focar 
nossa atenção em $\mathcal{J}_{\Delta}^{*}$. Primeiro notamos que $\mathcal{J}_{\Delta}^{*}$ é igual ao jacobiano obtido a partir do modelo em que nos restringimos apenas às opiniões em $\Delta$, mas em um ponto fixo em que nenhuma opinião foi extinta, ou seja se o modelo original tiver regra de confiança $\mathcal{R}$, então $\mathcal{J}_{\Delta}^{*}$ seria o jacobiano do modelo com regra $\mathcal{R}_{\Delta}$ em um ponto fixo em que todas as opiniões (que agora seriam apenas as em $\Delta$ ) sobrevivem. Por isso, iremos analisar essa parte do espectro assumindo que todas as opiniões sobrevivem. Porém como $\mathcal{R}_{\Delta}$ pode ter mais de uma componente, mesmo se $\mathcal{R}$ só tiver uma componente, iremos analisar os casos com uma e muitas componentes separadamente. Finalmente, $\mathcal{J}_{\Omega}^{*}$ será analisado de maneira mais detalhada na seção 4.2.3

Antes de prosseguir, lembramos que como vimos na seção introdutória sobre sistemas dinâmicos (seção 1.3), a conservação da soma das variáveis $\eta_{\sigma}$ deve introduzir um artefato de imersão. Mais precisamente, como

$$
\phi(\vec{\eta})=\sum_{\sigma} \eta_{\sigma}
$$

se conserva, então $\vec{\nabla} \phi=(1, \ldots, 1) \equiv \overrightarrow{1}$ deve ser um autovetor à esquerda com autovalor 0 para todos os pontos fixos e no nosso caso, como as segundas derivadas de $\phi$ se anulam no espaço de fase inteiro, segue da equação 1.3.6 que $\overrightarrow{1} \cdot \mathcal{J}_{\vec{F}}=\overrightarrow{0}$ no espaço de fase inteiro. Mais explicitamente

$$
\left(\overrightarrow{1} . \mathcal{J}_{\vec{F}}\right)_{\sigma}=\sum_{\sigma^{\prime}} \mathcal{J}_{\sigma^{\prime}, \sigma}^{*}=\sum_{\sigma^{\prime}} \frac{\partial}{\partial \eta_{\sigma}} F_{\sigma^{\prime}}=\frac{\partial}{\partial \eta_{\sigma}} \sum_{\sigma^{\prime}} F_{\sigma^{\prime}}=0 .
$$

O importante aqui é que todos os autovetores à direita que não são artefatos de imersão devem ser ortogonais a $\overrightarrow{1}$, ou seja, são vetores paralelos ao espaço de fase.

Por fim, notamos que $\vec{F}$ é uma função homogênea de grau 3 , implicando que

$$
\mathcal{J}_{\vec{F}} \cdot \vec{\eta}=3 \vec{F}=3 \dot{\vec{\eta}} \Rightarrow \mathcal{J}^{*} \cdot \vec{\eta}^{*}=\overrightarrow{0}
$$

de forma que o autovetor à direita correspondente ao artefato nos pontos fixos é $\vec{\eta}^{*}$.

\subsubsection{Regras de confiança com mais de uma componente}

Iremos começar a análise com o caso em que a regra tem mais de uma componente. A conclusão principal em que iremos chegar é que as componentes podem ser analisadas separadamente, com alguns outros detalhes do espectro podendo ser tratados da mesma forma que artefatos de imersão, faltando então apenas a análise do caso de uma componente, que será feito nas seções seguintes.

Seja $\mathcal{R}$ a nossa regra de confiança e sejam $\Delta_{1}, \Delta_{2}, \ldots, \Delta_{k}$ as suas componentes. Em um certo sentido, a regra restrita a cada uma dessas componentes se comporta como se fosse um modelo independente, pois as opiniões de uma componente não interagem com as outras. Podemos permutar $\mathcal{J}^{*}$ para: 


$$
\mathcal{J}^{*}=\left[\begin{array}{cccc}
\mathcal{J}_{\Delta_{1}}^{*} & 0 & \ldots & 0 \\
0 & \mathcal{J}_{\Delta_{2}}^{*} & \ldots & 0 \\
\vdots & \vdots & \ddots & \vdots \\
0 & 0 & \ldots & \mathcal{J}_{\Delta_{k}}^{*}
\end{array}\right]
$$

deixando nas primeiras linhas as coordenadas correspondentes às opiniões em $\Delta_{1}$, seguidas das correspondentes às que estão em $\Delta_{2}$ e assim por diante. De maneira análoga ao que aconteceu quando separamos $\Delta$ e $\Omega$ no caso geral, $\mathcal{J}_{\Delta_{i}}^{*}$ vai ser o jacobiano de um ponto fixo em que todas as opiniões coexistem e a regra é dada por $\mathcal{R}_{\Delta_{i}}$. Como $\mathcal{J}^{*}$ é bloco diagonal, basta saber o resultado para uma componente, para cada $\mathcal{J}_{\Delta_{i}}^{*}$ (o que será feito nas seções seguintes).

Iremos agora mostrar explicitamente que as equações para as diversas componentes podem ser separadas. No nosso caso, a equação 4.1.1 tem $k$ constantes de movimento:

$$
\eta_{\Delta_{i}}=\sum_{\sigma \in \Delta_{i}} \eta_{\sigma}, i=1, \ldots, k
$$

relacionadas por

$$
\sum_{i=1}^{k} \eta_{\Delta_{i}}=1
$$

Definindo

$$
\zeta_{\sigma}=\frac{\eta_{\sigma}}{\eta_{\Delta(\sigma)}}
$$

onde $\Delta(\sigma)$ é a componente à qual $\sigma$ pertence, segue que $\zeta_{\sigma}$ obedece a

$$
\dot{\zeta}_{\sigma}=\eta_{\Delta(\sigma)}^{2} \sum_{\sigma^{\prime} \in \Delta(\sigma)} \zeta_{\sigma} \zeta_{\sigma^{\prime}}\left(\zeta_{\sigma} p_{\sigma \rightarrow \sigma^{\prime}}-\zeta_{\sigma^{\prime}} p_{\sigma^{\prime} \rightarrow \sigma}\right)
$$

que é a equacão de campo médio a menos da escala de tempo ( 1 unidade de tempo da equação usual corresponde a $1 / \eta_{\Delta(\sigma)}^{2}$ unidades da equação 4.2 .12 , de forma que só faz sentido falar dela quando $\eta_{\Delta(\sigma)} \neq 0$ ), para o modelo com regra $\mathcal{R}_{\Delta(\sigma)}$, que é um caso com somente uma componente e tal que

$$
\sum_{\sigma^{\prime} \in \Delta(\sigma)} \zeta_{\sigma^{\prime}}=1
$$

Se para cada uma das $k$ componentes, $\vec{\zeta}_{i}$ for um ponto fixo do modelo com regra $\mathcal{R}_{\Delta_{i}}$, então todos os pontos do espaço de fase que correspondem aos mesmos $\vec{\zeta}_{i}$, através da 
equação 4.2.11, devem ser pontos fixos da regra $\mathcal{R}$ e esses pontos formam um hiperplano $\mathcal{F}\left(\vec{\zeta}_{1}, \vec{\zeta}_{2}, \ldots, \vec{\zeta}_{k}\right)$ definido por

$$
\mathcal{F}\left(\vec{\zeta}_{1}, \ldots, \vec{\zeta}_{k}\right)=\left\{\vec{\eta} \in \operatorname{Sim}_{M} \mid \eta_{\sigma}=\zeta_{\sigma} \eta_{\Delta(\sigma)}\right\}
$$

que forma uma variedade parametrizada pelos $\eta_{\Delta_{i}}$.

Para prosseguir em nossa análise, iremos encontrar, para cada conjunto $\left\{\vec{\zeta}_{i}\right\}$, a base que gera o hiperplano associado. Definindo

$$
\vec{u}_{1}=\left[\begin{array}{c}
\vec{\zeta}_{1} \\
\overrightarrow{0} \\
\vdots \\
\overrightarrow{0}
\end{array}\right], \vec{u}_{2}=\left[\begin{array}{c}
\overrightarrow{0} \\
\vec{\zeta}_{2} \\
\vdots \\
\overrightarrow{0}
\end{array}\right], \ldots, \vec{u}_{k}=\left[\begin{array}{c}
\overrightarrow{0} \\
\overrightarrow{0} \\
\vdots \\
\vec{\zeta}_{k}
\end{array}\right],
$$

onde o primeiro elemento é o bloco com as opiniões em $\Delta_{1}$, o seguinte tem as opiniões de $\Delta_{2}$ e assim por diante, até as opiniões de $\Delta_{k}$ (ou seja, as opiniões estão dispostas nesses vetores de acordo com a forma como elas aparecem na matriz jacobiana na equação 4.2.9), então os pontos de $\mathcal{F}\left(\vec{\zeta}_{1}, \ldots, \vec{\zeta}_{k}\right)$ são da forma

$$
\vec{\eta}^{*}=\sum_{i} \eta_{\Delta_{i}} \vec{u}_{i}, \text { com } \sum_{i} \eta_{\Delta_{i}}=1
$$

Como todos os $\vec{u}_{i}$ são ortogonais entre si, então a base $\left\{\vec{v}_{i} \mid \vec{v}_{i}=\vec{u}_{i}-\vec{u}_{k}, i=1, \ldots, k-1\right\}$, é linearmente independente e ela gera $\mathcal{F}$, pois

$$
\vec{\eta}^{*}=\vec{u}_{k}+\sum_{i=1}^{k-1} \eta_{\Delta_{i}} \vec{v}_{i} .
$$

Primeiro, isso implica que $\mathcal{F}$ é sempre uma variedade com $k-1$ dimensões. Segundo, esses vetores são paralelos a $\mathcal{F}$ e todos os pontos do hiperplano são pontos fixos, logo esperamos que eles sejam autovetores que correspondam a direções neutras, ao longo das quais não há movimento. Isso é uma consequência de podermos tratar essas direções como artefatos de imersão (ver seção 1.3 para mais detalhes). Mais precisamente, segue das equações 4.2.7 e 4.2.8 que $\vec{u}_{1}, \ldots, \vec{u}_{k}$ são autovetores com autovalor 0 à direita e que os duais (autovetores à esquerda) são respectivamente

$$
\left[\begin{array}{llll}
\overrightarrow{1} & \overrightarrow{0} & \ldots & \overrightarrow{0}
\end{array}\right],\left[\begin{array}{llll}
\overrightarrow{0} & \overrightarrow{1} & \ldots & \overrightarrow{0}
\end{array}\right], \ldots,\left[\begin{array}{llll}
\overrightarrow{0} & \overrightarrow{0} & \ldots & \overrightarrow{1}
\end{array}\right] .
$$

A equação de campo médio com os parâmetros que estamos utilizando tem as grandezas conservadas

$$
\phi_{i}(\vec{\eta})=\sum_{\sigma \in \Delta_{i}} \eta_{\sigma}
$$


e $\vec{\nabla} \phi_{1}=(\overrightarrow{1}, \overrightarrow{0}, \overrightarrow{0}, \ldots, \overrightarrow{0}), \vec{\nabla} \phi_{2}=(\overrightarrow{0}, \overrightarrow{1}, \overrightarrow{0}, \ldots, \overrightarrow{0}), \ldots, \vec{\nabla} \phi_{k}=(\overrightarrow{0}, \overrightarrow{0}, \overrightarrow{0}, \ldots, \overrightarrow{1})$, que são os autovetores à esquerda, formam uma base linearmente independente, de forma que podemos aplicar o tratamento apresentado na seção 1.3 e para analisar o fluxo restrito à variedade

$$
\left\{\vec{\eta} \in \operatorname{Sim}_{M} \mid \phi_{i}=\eta_{\Delta_{i}}\right\}
$$

esses autovalores nulos não precisam ser levados em conta. Antes de prosseguir, vamos lembrar que o que fizemos não prova que o hiperplano $\mathcal{F}$ não possa fazer parte de uma variedade de pontos fixos com mais dimensões ainda. No caso geral, precisariamos mostrar que os pontos fixos $\vec{\zeta}_{1}, \ldots, \vec{\zeta}_{k}$ são isolados. Na próxima seção, por exemplo, mostramos que um ponto fixo de coexistência (todas as opiniões sobrevivem) em um modelo com uma componente é sempre isolado, de forma que os pontos fixos de coexistência de um modelo de $k$ componentes aparecem em variedades de $k-1$ dimensões.

\subsubsection{Pontos fixos de coexistência}

Vamos considerar agora o caso em que todas as opiniões sobrevivem e $\mathcal{R}$ tem apenas uma componente. Ao invés de calcular os autovalores do jacobiano $\mathcal{J}^{*}$, nós iremos fazer uma transformação nessa matriz, para chegar em outra (que chamaremos de $A$ ) em que podemos descobrir o sinal da parte real dos autovalores e depois recuperar essa informação para a matriz original (o arcabouço necessário é desenvolvido em detalhe no apêndice A.2 e os teoremas desse apêndice serão frequentemente utilizados).

Sejam $\vec{\eta}^{*}$ o ponto fixo de coexistência que queremos analisar, $D=\operatorname{diag}\left(\vec{\eta}^{*}\right)$, a matriz diagonal em que os termos da diagonal principal são as coordenadas de $\vec{\eta}^{*}$ e $A=\mathcal{J}^{*} D+$ $\left(\mathcal{J}^{*} D\right)^{T}$. A matriz $A$ é simétrica real e, por causa das equações 4.2 .7 e 4.2 .8 , ela é tal que a soma de cada uma das linhas e de cada uma das colunas é 0 . Como

$$
A_{\sigma, \sigma^{\prime}}=\mathcal{J}_{\sigma, \sigma^{\prime}}^{*} \eta_{\sigma^{\prime}}^{*}+\mathcal{J}_{\sigma^{\prime}, \sigma}^{*} \eta_{\sigma}^{*}
$$

então se $\sigma \neq \sigma^{\prime}$, temos da equação 4.2.3 que $\mathcal{J}_{\sigma, \sigma^{\prime}}^{*}=\eta_{\sigma}^{* 2} p_{\sigma^{\prime} \rightarrow \sigma}-2 \eta_{\sigma}^{*} \eta_{\sigma^{\prime}}^{*} p_{\sigma \rightarrow \sigma^{\prime}}$, logo

$$
A_{\sigma, \sigma^{\prime}}=-\eta_{\sigma}^{*} \eta_{\sigma^{\prime}}^{*}\left(\eta_{\sigma}^{*} p_{\sigma^{\prime} \rightarrow \sigma}+\eta_{\sigma^{\prime}}^{*} p_{\sigma \rightarrow \sigma^{\prime}}\right) .
$$

Como $A_{\sigma, \sigma^{\prime}} \leq 0$, segue que $A$ sempre é uma $C_{0}$-matriz. No apêndice A.2, demonstramos o teorema A.2.17, que diz no nosso caso que se $A$ for irredutível (não pode ser permutada para uma forma bloco triangular), então todos os autovalores de $A$ são reais positivos, com a exceção de um autovalor nulo (com multiplicidade 1). Porém, o fato da regra $\mathcal{R}$ ter apenas uma componente implica que temos 2 casos, ou o modelo tem apenas uma opinião e nesse caso a matriz $A$ é nula, ou $A$ é irredutível.

Para ver que $A$ é sempre irredutível no caso com mais de uma opinião, primeiro notamos que se $\sigma \neq \sigma^{\prime}$, então $A_{\sigma, \sigma^{\prime}} \neq 0$ se e somente se $\sigma$ e $\sigma^{\prime}$ estão conectados no esqueleto nãodirecionado de $\mathcal{R}, \operatorname{Sk}(\mathcal{R})$. Além disso, como $\mathcal{R}$ tem apenas uma componente, $\operatorname{Sk}(\mathcal{R})$ é um 
grafo conectado e como $A$ é simétrica, uma permutação que a leve em uma forma bloco triangular a leva em uma forma bloco diagonal. Suponha então que $A$ não seja irredutível. Isso implica que deve existir uma permutação dela para

$$
\left[\begin{array}{cc}
A_{X, X} & 0 \\
0 & A_{Y, Y}
\end{array}\right]
$$

onde $A_{X, X}$ e $A_{Y, Y}$ são as matrizes com os termos $A_{\sigma, \sigma^{\prime}}$ onde respectivamente $\sigma, \sigma^{\prime} \in X \mathrm{e}$ $\sigma, \sigma^{\prime} \in Y, \operatorname{com} X, Y \neq \varnothing$. Mas então isso significa que $X$ e $Y$ não possuem conexões entre si em $\operatorname{Sk}(\mathcal{R})$, o que contradiz $\operatorname{Sk}(\mathcal{R})$ ser conectado.

Uma vez que estabelecemos que $A$ é irredutível, temos todas as propriedades do seu espectro (usando o teorema A.2.17). Agora é necessário traduzir o que esses resultados significam para o jacobiano $\mathcal{J}^{*}$. Para isso, usaremos nossa extensão do teorema de Johnson (teorema A.2.11). Esse teorema implica que se $A$ tem todos os autovalores positivos, com a exceção do 0 que tem multiplicidade algébrica 1 , então $\mathcal{J}^{*}$ tem todos os autovalores com parte real não-negativa e a soma das multiplicidades geométricas dos autovalores com parte real nula é menor ou igual a 1 . Como o 0 é um autovalor de $\mathcal{J}^{*}$ (por causa do artefato, devido à conservação da soma das variáveis) e todo autovalor tem multiplicidade geométrica maior ou igual a 1 , isso implica que todos os autovalores de $\mathcal{J}^{*}$ tem parte real positiva, com a exceção do 0 que tem multiplicidade geométrica 1. A diferença importante entre o resultado para a matriz $A$ e para o jacobiano $\mathcal{J}^{*}$ é que em um determinamos a multiplicidade algébrica do 0 (a multiplicidade do autovalor na equação característica) e no outro, determinamos a multiplicidade geométrica (a dimensão do espaço gerado pelos autovetores). Essas 2 multiplicidades são sempre iguais se a matriz é diagonalizável, porém a forma como escrevemos $\mathcal{J}^{*}$ (em termos de uma solução hipotética) torna bem difícil determinar se ele é diagonalizável ou não. Além disso, no caso geral, a multiplicidade geométrica é menor ou igual à algébrica e o nosso argumento de que podemos ignorar o 0 na análise de estabilidade linear, por ser um artefato, assume que a multiplicidade algébrica é 1. Por essa razão, vamos analisar as submatrizes principais com ordem $M-1 \mathrm{de} \mathcal{J}^{*}$, para utilizar o teorema A.2.16 e mostrar que a multiplicidade algébrica é 1.

O que precisamos provar é que se $\mathcal{J}^{*(\sigma)}$ for a submatriz principal de $\mathcal{J}^{*}$ obtida com a remoção da linha e da coluna $\sigma$, então

$$
\sum_{\sigma} \operatorname{det}\left(\mathcal{J}^{*(\sigma)}\right) \neq 0
$$

Para tanto, iremos mostrar que $\operatorname{det}\left(\mathcal{J}^{*(\sigma)}\right) \geq 0$ para todo $\sigma$ e que $\operatorname{det}\left(\mathcal{J}^{*(\sigma)}\right) \neq 0$ para algum $\sigma$. Se definirmos $A^{(\sigma)}$ e $D^{(\sigma)}$ a partir de $A$ e $D$, da mesma forma que definimos $\mathcal{J}^{*(\sigma)}$ a partir de $\mathcal{J}^{*}$, então notamos que $A^{(\sigma)}=\mathcal{J}^{*(\sigma)} D^{(\sigma)}+\left(\mathcal{J}^{*(\sigma)} D^{(\sigma)}\right)^{T}$. Isso implica que podemos fazer em $A^{(\sigma)}$ uma análise parecida com a que fizemos em $A$, para obter as informações que queremos sobre $\mathcal{J}^{*(\sigma)}$.

Lembramos primeiro que $A_{\sigma, \sigma^{\prime}} \neq 0$ se e somente se $\sigma$ e $\sigma^{\prime}$ estão conectados em $\operatorname{Sk}(\mathcal{R})$ 
e como $\operatorname{Sk}(\mathcal{R})$ é conectado, então todo $\sigma$ se conecta com algum $\sigma^{\prime} \neq \sigma$. Isso é equivalente a dizer que para todo $\sigma$ existe um $\sigma^{\prime}$ tal que $A_{\sigma, \sigma^{\prime}}<0$. Como a soma dos termos de cada uma das linhas de $A$ é 0 , então a soma dos termos da linha (e da coluna) $\sigma^{\prime}$ é $-A_{\sigma, \sigma^{\prime}}$. Isso tem 2 consequências, todos os $A^{(\sigma)}$ serão $C_{0}$-matrizes e logo positivas semidefinidas e todos os $A^{(\sigma)}$ tem alguma linha cujos termos tem uma soma positiva. Como os $A^{(\sigma)}$ são positivos semidefinidos, então usando o teorema de Johnson extendido, chegamos a conclusão que os $\mathcal{J}^{*(\sigma)}$ também são positivos semidefinidos e como os $\mathcal{J}^{*(\sigma)}$ são matrizes reais, segue (essencialmente uma consequência do teorema A.2.9) que $\operatorname{det}\left(\mathcal{J}^{*(\sigma)}\right) \geq 0$.

Para provar que $\operatorname{det}\left(\mathcal{J}^{*(\sigma)}\right) \neq 0$ para algum $\sigma$, basta mostrar que algum $A^{(\sigma)}$ é positivo definido e usar o teorema de Johnson, para mostrar que o $\mathcal{J}^{*(\sigma)}$ correspondente também é positivo definido e logo pelo teorema A.2.9, tem $\operatorname{det}\left(\mathcal{J}^{*(\sigma)}\right)>0$. Iremos usar o teorema A.2.14, que junto com as outras propriedades que provamos, implica que se $A^{(\sigma)}$ é irredutível, então ele é positivo definido (e logo, como já vimos, $\operatorname{det}\left(\mathcal{J}^{*(\sigma)}\right)>0$ ). Por outro lado, como $A$ é irredutível, a existência de um $A^{(\sigma)}$ irredutível é assegurada pelo teorema A.2.15 desde que algum dos termos da diagonal principal de $A$ seja não-nulo. Como para todo $\sigma$, existe $\sigma^{\prime} \neq \sigma$ tal que $A_{\sigma, \sigma^{\prime}}<0$ e a soma de cada linha de $A$ é 0 , então $A_{\sigma, \sigma}>0$ para todo $\sigma$, ou seja, todos os termos da diagonal principal de $A$ são positivos, o que termina a prova que a multiplicidade algébrica do 0 é 1 para $\mathcal{J}^{*}$, correspondendo então ao artefato de imersão causado pela conservação de $\sum_{\sigma} \eta_{\sigma}$. Como todos os outros autovalores tem parte real positiva, podemos concluir que todos os pontos fixos, do caso de uma componente, em que todas as opiniões coexistem, devem ser nós instáveis. Além disso, isso implica que podemos usar o teorema da função implícita para mostrar que esses pontos fixos devem ser isolados (eles são os únicos pontos fixos em alguma vizinhança aberta deles).

É importante salientar alguns pontos sobre o caso em que $\mathcal{R}$ possui mais de uma componente (lembramos que esse caso vai ser importante quando $\Omega \neq \varnothing$, pois mesmo se $\mathcal{R}$ só tiver uma componente, não há nenhuma garantia que $\mathcal{R}_{\Delta}$ também só tenha uma). Nosso resultado para uma componente implica que, se $\mathcal{R}$ tem $k$ componentes, $\mathcal{J}^{*}$ é positiva semidefinida, o 0 é o único autovalor com parte real nula e ele tem multiplicidade algébrica e geométrica iguais a $k$. Além disso, o isolamento dos pontos fixos mostra que eles aparecem em variedades com $k-1$ dimensões, que são hiperplanos dados pela equação 4.2.13. Finalmente, nosso resultado implica que esses hiperplanos não são Lyapunov estáveis, a menos que $k=M$.

\subsubsection{Pontos fixos em que opiniões são extintas}

Agora examinamos o caso em que nem todas as opiniões sobrevivem no ponto fixo, o que corresponde ao caso mais geral. Iremos nos restringir ao caso em que a regra de confiança $\mathcal{R}$ tem uma componente, pois como vimos na seção 4.2.1, diferentes componentes equivalem apenas a modelos independentes entre si. Porém, como $\mathcal{R}_{\Delta}$ terá muitas componentes em vários casos, iremos fazer uso do caso em que todas as opiniões coexistem, para mais de uma componente. Iremos mais uma vez chamar de $\vec{\eta}^{*}$ o ponto fixo que estamos analisando. Como temos opiniões que são extintas, então $\Omega \neq \varnothing$ e o jacobiano $\mathcal{J}^{*}$ tem a forma dada 
pela equação 4.2.4:

$$
\mathcal{J}^{*}=\left[\begin{array}{cc}
\mathcal{J}_{\Delta}^{*} & \mathcal{N} \\
0 & \mathcal{J}_{\Omega}^{*}
\end{array}\right]
$$

Os resultados para pontos fixos de coexistência em $\mathcal{R}_{\Delta}$ valem automaticamente e o resto do espectro é dado pela matriz $\mathcal{J}_{\Omega}^{*}$. Mas como

$$
\left(\mathcal{J}_{\Omega}^{*}\right)_{\sigma, \sigma^{\prime}}=-\delta_{\sigma, \sigma^{\prime}} \sum_{\sigma^{\prime \prime}} \eta_{\sigma^{\prime \prime}}^{* 2} p_{\sigma \rightarrow \sigma^{\prime \prime}}
$$

então $\mathcal{J}_{\Omega}^{*}$ é uma matriz diagonal e seus autovalores são

$$
\lambda_{\sigma}=-\sum_{\sigma^{\prime}} \eta_{\sigma^{\prime}}^{* 2} p_{\sigma \rightarrow \sigma^{\prime}}
$$

Se $\lambda_{\sigma} \neq 0$ para todas as opiniões em $\Omega$, então temos um retrato completo das trajetórias na vizinhança de $\vec{\eta}^{*}$ (podemos determinar o sinal da parte real dos autovalores de $\mathcal{J}^{*}$ e somente artefatos de imersão possuem autovalores com parte real nula). Porém, existe a possibilidade de $\lambda_{\sigma}$ ser nulo. Mais precisamente

$$
\lambda_{\sigma}=0 \Leftrightarrow \eta_{\sigma^{\prime}}^{*} p_{\sigma \rightarrow \sigma^{\prime}}=0 \forall \sigma^{\prime} \Leftrightarrow p_{\sigma \rightarrow \sigma^{\prime}}=0 \forall \sigma^{\prime} \in \Delta \Leftrightarrow \sigma \notin \Delta_{-}(\mathcal{R})
$$

onde lembramos que $\Delta_{-}(\mathcal{R})$ denota o conjunto predecessor de $\Delta$, com respeito ao grafo da regra de confiança, $\mathcal{R}$ (ver a seção 1.2 para as definições relevantes). Ou seja, se nenhuma das opiniões que sobrevivem consegue convencer $\sigma$, então $\lambda_{\sigma}=0$. Além disso, como $\lambda_{\sigma}$ é sempre menor ou igual a 0 , isso implica que se cada uma das opiniões que são extintas puder ser convencida (no sentido que isso é permitido pela regra de confiança) por alguma das opiniões sobreviventes, então a variedade $\mathcal{M}_{\Delta}$, definida por

$$
\mathcal{M}_{\Delta}=\left\{\vec{\eta} \in \operatorname{Sim}_{M} \mid \eta_{\sigma}=0 \forall \sigma \in \bar{\Delta}\right\}
$$

é atrativa em uma vizinhança de $\vec{\eta}^{*}$. A interpretação disso é intuitiva: se pegarmos o estado $\vec{\eta}^{*}$ e "adicionarmos uma pequena quantidade" das opiniões que fazem parte de $\Omega$, rapidamente elas serão extintas pelas opiniões em $\Delta$, mas isso só acontece se todas elas puderem ser eliminadas por opiniões em $\Delta$.

Voltando ao caso em que $\lambda_{\sigma}=0$, o autovetor à direita correspondente será $\hat{e}_{\sigma}-\vec{\eta}^{*}$ (onde $\hat{e}_{\sigma}$ é o vetor que tem todas as componentes nulas, exceto a que corresponde a $\sigma$, que é igual a 1). Precisamos ir além da análise de estabilidade linear nesse caso. Fazendo a expansão em Taylor da equação 4.1.1 vem que se $\vec{\eta}=\vec{\eta}^{*}+\vec{\epsilon}$, então

$$
\dot{\epsilon}_{\sigma}=F_{\sigma}\left(\vec{\eta}^{*}\right)+\left(\frac{\partial F_{\sigma}}{\partial \eta_{\sigma^{\prime}}}\left(\vec{\eta}^{*}\right)\right) \epsilon_{\sigma^{\prime}}+\frac{1}{2}\left(\frac{\partial^{2} F_{\sigma}}{\partial \eta_{\sigma^{\prime}} \partial \eta_{\sigma^{\prime \prime}}}\left(\vec{\eta}^{*}\right)\right) \epsilon_{\sigma^{\prime}} \epsilon_{\sigma^{\prime \prime}}+\mathcal{O}\left(\|\vec{\epsilon}\|^{3}\right),
$$


usando a notação de Einstein (ou seja, se 2 índices aparecem repetidos em um termo, uma soma sobre aquele índice está subentendida: $\left.x_{i} y_{i}=\sum_{i} x_{i} y_{i}\right)$. Vamos agora calcular o termo de segunda ordem:

$$
\begin{gathered}
\frac{1}{2} \frac{\partial^{2} F_{\sigma}}{\partial \eta_{\sigma^{\prime}} \partial \eta_{\sigma^{\prime \prime}}}=\frac{1}{2} \frac{\partial}{\partial \eta_{\sigma^{\prime \prime}}}\left(\mathcal{J}_{\vec{F}}\right)_{\sigma, \sigma^{\prime}}= \\
=\frac{1}{2} \frac{\partial}{\partial \eta_{\sigma^{\prime \prime}}}\left(\delta_{\sigma, \sigma^{\prime}} \sum_{\sigma^{\prime \prime \prime}}\left(2 \eta_{\sigma} \eta_{\sigma^{\prime \prime \prime}} p_{\sigma^{\prime \prime \prime} \rightarrow \sigma}-\eta_{\sigma^{\prime \prime \prime}}^{2} p_{\sigma \rightarrow \sigma^{\prime \prime \prime}}\right)+\eta_{\sigma}^{2} p_{\sigma^{\prime} \rightarrow \sigma}-2 \eta_{\sigma} \eta_{\sigma^{\prime}} p_{\sigma \rightarrow \sigma^{\prime}}\right)= \\
=\delta_{\sigma, \sigma^{\prime}} \sum_{\sigma^{\prime \prime \prime}}\left(\left(\frac{\partial \eta_{\sigma}}{\partial \eta_{\sigma^{\prime \prime}}} \eta_{\sigma^{\prime \prime \prime}}+\eta_{\sigma} \frac{\partial \eta_{\sigma^{\prime \prime \prime}}}{\partial \eta_{\sigma^{\prime \prime}}}\right) p_{\sigma^{\prime \prime \prime} \rightarrow \sigma}-\eta_{\sigma^{\prime \prime \prime}} \frac{\partial \eta_{\sigma^{\prime \prime \prime}}}{\partial \eta_{\sigma^{\prime \prime}}} p_{\sigma \rightarrow \sigma^{\prime \prime \prime}}\right)+ \\
+\eta_{\sigma} \frac{\partial \eta_{\sigma}}{\partial \eta_{\sigma^{\prime \prime}}} p_{\sigma^{\prime} \rightarrow \sigma}-\left(\frac{\partial \eta_{\sigma}}{\partial \eta_{\sigma^{\prime \prime}}} \eta_{\sigma^{\prime}}+\eta_{\sigma} \frac{\partial \eta_{\sigma^{\prime}}}{\partial \eta_{\sigma^{\prime \prime}}}\right) p_{\sigma \rightarrow \sigma^{\prime}}= \\
=\delta_{\sigma, \sigma^{\prime}} \sum_{\sigma^{\prime \prime \prime}}\left(\left(\delta_{\sigma, \sigma^{\prime \prime}} \eta_{\sigma^{\prime \prime \prime}}+\eta_{\sigma} \delta_{\sigma^{\prime \prime \prime}, \sigma^{\prime \prime}}\right) p_{\sigma^{\prime \prime \prime} \rightarrow \sigma}-\eta_{\sigma^{\prime \prime \prime}} \delta_{\sigma^{\prime \prime \prime}, \sigma^{\prime \prime}} p_{\sigma \rightarrow \sigma^{\prime \prime \prime}}\right)+ \\
+\eta_{\sigma} \delta_{\sigma, \sigma^{\prime \prime}} p_{\sigma^{\prime} \rightarrow \sigma}-\left(\delta_{\sigma, \sigma^{\prime \prime}} \eta_{\sigma^{\prime}}+\eta_{\sigma} \delta_{\sigma^{\prime}, \sigma^{\prime \prime}}\right) p_{\sigma \rightarrow \sigma^{\prime}}=\delta_{\sigma, \sigma^{\prime}, \sigma^{\prime \prime}} \sum_{\sigma^{\prime \prime \prime}} \eta_{\sigma^{\prime \prime \prime}} p_{\sigma^{\prime \prime \prime} \rightarrow \sigma}+ \\
+\delta_{\sigma, \sigma^{\prime}}\left(\eta_{\sigma} p_{\sigma^{\prime \prime} \rightarrow \sigma}-\eta_{\sigma^{\prime \prime}} p_{\sigma \rightarrow \sigma^{\prime \prime}}\right)+\delta_{\sigma, \sigma^{\prime \prime}}\left(\eta_{\sigma} p_{\sigma^{\prime} \rightarrow \sigma}-\eta_{\sigma^{\prime}} p_{\sigma \rightarrow \sigma^{\prime}}\right)-\delta_{\sigma^{\prime}, \sigma^{\prime \prime}} \eta_{\sigma} p_{\sigma \rightarrow \sigma^{\prime}}
\end{gathered}
$$

Para fazer a análise de estabilidade, precisamos estudar as trajetórias tangentes ao espaço $W_{n}$ gerado pelos autovetores $\vec{v}_{\sigma}=\hat{e}_{\sigma}-\vec{\eta}^{*}$, ou seja, precisamos considerar

$$
\vec{\epsilon}=\sum_{\sigma} \alpha_{\sigma} \vec{v}_{\sigma}
$$

onde a soma é sobre os elementos $\sigma$ de $\Omega$, com $\lambda_{\sigma}=0$, e estudar como os $\alpha_{\sigma}$ evoluem no tempo. O teorema da variedade central garante que essa análise é consistente (ele essencialmente diz no nosso caso que existe uma variedade suave, não necessariamente única, composta de trajetórias do sistema e que é tangente a $W_{n}$ em $\vec{\eta}^{*}$, o enunciado exato se encontra no apêndice A.4), de forma que se $\sigma \in \Omega$ e $\lambda_{\sigma}=0$, então como o único desses autovetores que tem coordenada $\sigma$ não-nula é o $\vec{v}_{\sigma}$, basta estudar a evolução dessas coordenadas de $\vec{\epsilon}$. Voltando para a equação 4.2.21, se $\sigma \in \Omega$ e $\lambda_{\sigma}=0$, então a expressão se simplifica e temos

$$
F_{\sigma}\left(\vec{\eta}^{*}\right)=0, \frac{\partial F_{\sigma}}{\partial \eta_{\sigma^{\prime}}}\left(\vec{\eta}^{*}\right)=0 \text { e } \frac{1}{2} \frac{\partial^{2} F_{\sigma}}{\partial \eta_{\sigma^{\prime}} \partial \eta_{\sigma^{\prime \prime}}}\left(\vec{\eta}^{*}\right)=\delta_{\sigma, \sigma^{\prime}, \sigma^{\prime \prime}} \sum_{\sigma^{\prime \prime \prime}} \eta_{\sigma^{\prime \prime \prime}}^{*} p_{\sigma^{\prime \prime \prime} \rightarrow \sigma},
$$

pois $\eta_{\sigma^{\prime}}^{*} p_{\sigma \rightarrow \sigma^{\prime}}=0 \forall \sigma^{\prime}$ e $\eta_{\sigma}^{*}=0$. Além disso, como $\epsilon_{\sigma}=\eta_{\sigma}$ para essas opiniões, temos

$$
\dot{\eta}_{\sigma} \simeq \mu_{\sigma} \eta_{\sigma}^{2}, \text { onde } \mu_{\sigma} \equiv \sum_{\sigma^{\prime}} \eta_{\sigma^{\prime}}^{*} p_{\sigma^{\prime} \rightarrow \sigma}
$$


na vizinhança do ponto fixo. Como a solução da equação 4.2.22 é

$$
\eta_{\sigma}(t)=\frac{\eta_{\sigma o}}{1-\mu_{\sigma} \eta_{\sigma o} t},
$$

então se $\mu_{\sigma}>0$ para alguma opinião de $\Omega$ tal que $\lambda_{\sigma}=0$, as trajetórias nas redondezas do ponto fixo são repelidas da variedade $\eta_{\sigma}=0$ (aonde o ponto fixo se encontra). Portanto se $\lambda_{\sigma}=0$ e $\mu_{\sigma}>0$ para algum $\sigma$, temos que o ponto $\vec{\eta}^{*}$ não é Lyapunov estável. Como $\mu_{\sigma} \geq 0$, então $\mu_{\sigma}>0$ se e somente se $\eta_{\sigma^{\prime}}^{*} p_{\sigma^{\prime} \rightarrow \sigma} \neq 0$ para algum $\sigma^{\prime}$, o que é equivalente a dizer que $p_{\sigma^{\prime} \rightarrow \sigma} \neq 0$ para algum $\sigma^{\prime} \in \Delta$, ou seja, $\sigma$ é capaz de convencer alguma das opiniões sobreviventes do ponto fixo. Isso fornece uma explicação intuitiva para a instabilidade, pois se $\lambda_{\sigma}=0$ e $\mu_{\sigma}>0$, então $\sigma$ consegue convencer uma das opiniões sobreviventes, porém nenhuma das sobreviventes consegue convencer $\sigma$, de forma que se tomarmos um estado próximo de $\vec{\eta}^{*} \operatorname{com} \eta_{\sigma} \neq 0$, a opinião $\sigma$ vai lentamente convencer agentes com opinião $\Delta$, sem perder agentes para outras opiniões.

Finalmente, vamos analisar o caso em que $\lambda_{\sigma}=0$ e $\mu_{\sigma}=0$. Vamos chamar de $\omega$ o conjunto de opiniões em $\Omega$, tais que $\lambda_{\sigma}$ e $\mu_{\sigma}$ são nulos. Primeiro notamos que

$$
\mu_{\sigma}=0 \Leftrightarrow \eta_{\sigma^{\prime}}^{*} p_{\sigma^{\prime} \rightarrow \sigma}=0 \forall \sigma^{\prime} \Leftrightarrow p_{\sigma^{\prime} \rightarrow \sigma}=0 \forall \sigma^{\prime} \in \Delta \Leftrightarrow \sigma \notin \Delta_{+}(\mathcal{R}),
$$

que resume parte da nossa discussão anterior. As equações 4.2.19 e 4.2.24 implicam que as opiniões em $\omega$ são as opiniões que não sobrevivem e não interagem com as opiniões sobreviventes. Ou seja, $\omega \cap \Gamma_{\Delta}(\mathcal{R})=\varnothing$. Como $\mathcal{R}$ tem apenas uma componente, isso implica que $\omega \neq \Omega$. Suponha que $\lambda_{\sigma} \neq 0 \forall \sigma \in \Omega-\omega$. Defina então

$$
\Lambda \equiv \max _{\sigma \in \Omega-\omega} \lambda_{\sigma} \text { e } \eta_{\omega}=\sum_{\sigma \in \omega} \eta_{\sigma}
$$

Segue que $\Lambda<0$ e que a evolução temporal de $\eta_{\omega}$ é

$$
\dot{\eta}_{\omega}=\sum_{\sigma \in \omega} \sum_{\sigma^{\prime} \in \Omega-\omega} \eta_{\sigma} \eta_{\sigma^{\prime}}\left(\eta_{\sigma} p_{\sigma^{\prime} \rightarrow \sigma}-\eta_{\sigma^{\prime}} p_{\sigma \rightarrow \sigma^{\prime}}\right)
$$

Na vizinhança de $\vec{\eta}^{*}$ temos

$$
\eta_{\sigma} \simeq \eta_{\sigma \mathrm{o}} e^{\lambda_{\sigma} t} \forall \sigma \in \Omega-\omega,
$$

de forma que eu posso escrever a seguinte aproximação para a evolução temporal de $\eta_{\omega}$ :

$$
\dot{\eta}_{\omega} \simeq \sum_{\sigma \in \omega} \sum_{\sigma^{\prime} \in \Omega-\omega}\left(\eta_{\sigma^{\prime} \mathrm{o}} e^{\lambda_{\sigma^{\prime}} t} \eta_{\sigma}^{2} p_{\sigma^{\prime} \rightarrow \sigma}-\eta_{\sigma^{\prime} \mathrm{o}}^{2} e^{2 \lambda_{\sigma^{\prime}} t} \eta_{\sigma} p_{\sigma \rightarrow \sigma^{\prime}}\right)
$$

A qualidade dessa aproximação depende apenas de quão próxima a condição inicial está do ponto fixo estudado, logo para estabelecer a estabilidade, basta analisar qual das seguintes situações acontece: 
- Se $\eta_{\omega}$ se torna arbitrariamente próximo de 0 para uma condição inicial suficientemente próxima de $\vec{\eta}^{*}$, temos estabilidade assintótica.

- Se $\eta_{\omega}$ permanece para sempre, menor que uma constante $\epsilon>0$, sempre que a distância entre $\vec{\eta}^{*}$ e a condição incial for menor que $\delta(\epsilon)$, temos estabilidade de Lyapunov.

Podemos deduzir da aproximação 4.2.26 as seguintes desigualdades:

$$
\begin{gathered}
-\sum_{\sigma \in \omega} \sum_{\sigma^{\prime} \in \Omega-\omega} \eta_{\sigma^{\prime} \mathrm{o}}^{2} e^{2 \lambda_{\sigma^{\prime}} t} \eta_{\sigma} p_{\sigma \rightarrow \sigma^{\prime}} \leq \dot{\eta}_{\omega} \leq \sum_{\sigma \in \omega} \sum_{\sigma^{\prime} \in \Omega-\omega} \eta_{\sigma^{\prime} \mathrm{o}} e^{\lambda_{\sigma^{\prime}} t} \eta_{\sigma}^{2} p_{\sigma^{\prime} \rightarrow \sigma} \Rightarrow \\
-\sum_{\sigma \in \omega} \sum_{\sigma^{\prime} \in \Omega-\omega} e^{2 \lambda_{\sigma^{\prime}} t} \eta_{\sigma} \leq \dot{\eta}_{\omega} \leq \sum_{\sigma \in \omega} \sum_{\sigma^{\prime} \in \Omega-\omega} e^{\lambda_{\sigma^{\prime}} t} \eta_{\sigma} \Rightarrow \\
-\sum_{\sigma \in \omega} \sum_{\sigma^{\prime} \in \Omega-\omega} e^{2 \Lambda t} \eta_{\sigma} \leq \dot{\eta}_{\omega} \leq \sum_{\sigma \in \omega} \sum_{\sigma^{\prime} \in \Omega-\omega} e^{\Lambda t} \eta_{\sigma} \Rightarrow \\
-|\Omega-\omega| e^{2 \Lambda t} \eta_{\omega} \leq \dot{\eta}_{\omega} \leq|\Omega-\omega| e^{\Lambda t} \eta_{\omega} \Rightarrow \\
-|\Omega-\omega| e^{2 \Lambda t} \leq \frac{\mathrm{d}}{\mathrm{d} t} \ln \left(\eta_{\omega}\right) \leq|\Omega-\omega| e^{\Lambda t} .
\end{gathered}
$$

Finalmente, integrando no tempo e tomando o limite $t \rightarrow \infty$ vem

$$
\eta_{\omega 0} e^{|\Omega-\omega| / 2 \Lambda} \leq \eta_{\omega} \leq \eta_{\omega_{0}} e^{-|\Omega-\omega| / \Lambda}
$$

Como $\eta_{\omega}$ possui o minorante $\eta_{\omega o} e^{|\Omega-\omega| / 2 \Lambda}$ que é maior que 0 para todo $\eta_{\omega o} \neq 0$, então não temos estabilidade assintótica. Porém, $\eta_{\omega}$ possui o majorante $\eta_{\omega_{0}} e^{-|\Omega-\omega| / \Lambda}$ que pode ser feito arbitrariamente pequeno, tomando uma condição inicial arbitrariamente próxima de $\vec{\eta}^{*}$, de forma que o ponto fixo é Lyapunov estável. Em outras palavras, a variedade $\eta_{\omega}=0$ não repele as trajetórias, nem as atrai. Para entender o que está acontecendo, lembramos que $\mathcal{R}_{\omega}$ possui pelo menos um ponto fixo $\vec{x}$ (os estados de consenso são pontos fixos triviais de todas as regras de confiança) e que $\Delta$ e $\omega$ estão desconectados entre si em $\mathcal{R}$ (mesmo que eles façam parte da mesma componente), de forma que em $\mathcal{R}_{\Delta \cup \omega}, \Delta$ e $\omega$ funcionam como componentes diferentes (lembramos que ambos podem ser a união de várias componentes). Segue dos resultados que obtivemos na seção 4.2.1 que $\vec{x} \eta_{\omega}+\vec{\eta}^{*}\left(1-\eta_{\omega}\right)$ também é um ponto fixo de $\mathcal{R}$ (aqui $\vec{x}$ é obtido pegando as coordenadas do ponto fixo de $\mathcal{R}_{\omega}$ e atribuindo 0 às coordenadas que não correspondam a opiniões em $\omega$ ). Além disso,

$$
\vec{x} \eta_{\omega}+\vec{\eta}^{*}\left(1-\eta_{\omega}\right)=\vec{\eta}^{*}+\sum_{\sigma \in \omega} x_{\sigma} \vec{v}_{\sigma}
$$

de forma que essa linha de pontos fixos está contida no espaço $W_{n}$, gerado pelos autovetores com parte real nula (mais precisamente, o subespaço gerado pelos $\vec{v}_{\sigma}$ em que $\sigma \in \omega$ ).

$\mathrm{O}$ aspecto mais importante dos resultados que obtivemos é que as propriedades de estabilidade de um ponto fixo só dependem de quais das opiniões sobrevivem nele (o conjunto 
$\Delta$ ) e de quais opiniões são capazes de convencer quais opiniões (o esqueleto direcionado da regra de confiança, $\operatorname{Sk}(\mathcal{R})$ )

Podemos juntar todas as nossas conclusões para determinar quando um ponto fixo é um atrator. Primeiro, o conjunto $\Delta$ de opiniões que sobrevivem deve ser tal que $\mathcal{R}_{\Delta}$ tenha $|\Delta|$ componentes, o que corresponde a dizer que ele é independente em $\mathcal{R}$. Caso contrário, nossos resultados da seção 4.2.2 implicam que o jacobiano teria algum autovalor com parte real positiva. Isso também implica que a variedade em que as opiniões em $\Delta$ sobrevivem,

$$
\mathcal{M}_{\Delta}=\left\{\vec{\eta} \in \operatorname{Sim}_{M} \mid \eta_{\sigma}=0 \forall \sigma \in \bar{\Delta}\right\},
$$

que haviamos definido na equação 4.2.20, é constítuida inteiramente de pontos fixos (a existência desses pontos fixos depende apenas de $\Delta$ ser independente, de forma que mesmo sem estudar quando um ponto fixo com determinadas propriedades existe no caso geral, que será o assunto da próxima seção, podemos determinar todos os atratores estáticos do modelo). Os nossos resultados implicam que as propriedades de estabilidade de todos os pontos dessa variedade são iguais e logo, devemos estabelecer quando $\mathcal{M}_{\Delta}$ é um atrator. Para que ela seja um atrator, precisamos que $\omega=\varnothing$, pois senão não temos estabilidade assintótica. Além disso, como vimos, se $\omega \neq \varnothing, \Delta$ e $\omega$ não possuem conexões entre si e logo $\mathcal{M}_{\Delta \cup\{\sigma\}}$ também seria uma variedade de pontos fixos para todo $\sigma \in \omega$. Obviamente, se temos $\omega=\varnothing$, então todos os $\lambda_{\sigma}$ devem ser não-nulos, pois senão teríamos direções instáveis. Isso equivale a $\Omega \subseteq \Delta_{-}$. Como $\Omega=\bar{\Delta}$, então $\bar{\Delta} \subseteq \Delta_{-}$. Mas $\Delta$ é independente, implicando $\Delta \cap \Delta_{-}=\varnothing \Rightarrow \Delta_{-} \subseteq \bar{\Delta}$ e $\log \mathrm{\Delta}=\Delta_{-}$. Porém, como provamos no teorema A.1.1, $\bar{\Delta}=\Delta_{-}$sempre implica que $\Delta$ é independente maximal. Logo $\mathcal{M}_{\Delta}$ é um atrator se e somente se $\Delta$ é tal que $\bar{\Delta}=\Delta_{-}(\mathcal{R})$ e para encontrar os atratores na prática, primeiro encontramos todos os conjuntos independentes maximais e testamos $\bar{\Delta}=\Delta_{-}(\mathcal{R})$ para eles. Uma consequência que deve ser levada em conta, para modelos com números maiores de opiniões, é que dependendo do tipo de regra de confiança, o número de atratores diferentes cresce exponencialmente com o número de opiniões $M$ (isso acontece, por exemplo, se o esqueleto da regra de confiança for um ciclo em que todas as arestas são duplamente conectadas. Mesmo assim, no caso geral, a base dessa exponencial é sempre limitada a $\sqrt[3]{3} \simeq 1.442$ ).

\subsection{Existência dos pontos fixos}

Na seção anterior, nós mostramos como obter as propriedades de estabilidade de um ponto fixo, dadas apenas quais são as opiniões que sobrevivem nele e qual a regra de confiança que está sendo usada e usamos esses resultados para determinar quais são os atratores estáticos do modelo. Por outro lado, não nos preocupamos em determinar quantos pontos fixos de cada tipo existem (nem mesmo quais de fato existem). Para fazer essa análise, vamos primeiro considerar uma regra de confiança que possui um esqueleto direcionado completo, em outras palavras, todas opiniões podem convencer todas as outras. Iremos primeiro mostrar que, nesse caso, o campo $\vec{F}$ possui propriedades que nos permitem usar o teorema de 
Poincaré-Hopf, que estabelece conexões entre as propriedades topológicas de uma variedade com as propriedades algébricas de campos suaves definidos nelas. Esse teorema, junto com as propriedades que estabelecemos na seção 4.2.2 para todos os pontos fixos de coexistência, nos permite provar que existe sempre exatamente um ponto fixo de coexistência, quando o esqueleto é completo. Depois, iremos usar os resultados que obtivemos para pontos fixos em que opiniões são extintas, junto com o fato que todas as regras de confiança podem ser levadas em uma regra com esqueleto direcionado completo, através de uma perturbação infinitesimal de seus parâmetros, para determinar em quais regras com uma componente temos pontos fixos de coexistência (com o auxílio do teorema da função implícita). Finalmente, usamos os resultados da seção 4.2.1 para estudar os pontos fixos de coexistência no caso geral; lembrando que as trajetórias de $\mathcal{M}_{\Delta}$ em um modelo com regra $\mathcal{R}$ são equivalentes às de um modelo com regra $\mathcal{R}_{\Delta}$, iremos extender os resultados para pontos fixos em que apenas as opiniões em $\Delta$ sobrevivem.

Os resultados que obtivemos na seção 4.2.2 implicam que, se temos um ponto fixo de coexistência, em uma regra com esqueleto direcionado completo (ou seja, uma regra de confiança em que $p_{\sigma \rightarrow \sigma^{\prime}} \neq 0 \forall \sigma \neq \sigma^{\prime}$ ), então esse ponto seria isolado e um nó instável; e pela nossa definição de índice na seção 1.3, o índice desse ponto fixo seria 1. Para determinar que, nesse caso, existe um único ponto fixo de coexistência, iremos usar o teorema de PoincaréHopf (cujo enunciado pode ser encontrado no apêndice A.4, teorema A.4.1). Aplicado ao nosso problema, esse teorema nos diz que, se encontrarmos uma subvariedade $\mathcal{S}$ de $\operatorname{Sim}_{M}{ }^{1}$, tal que o campo $\vec{F}$, que define a derivada no tempo de $\vec{\eta}$, aponte para fora ao longo de toda a borda, então a soma dos índices dos pontos fixos de $\vec{F}$ que estão em $\mathcal{S}$ é igual à característica de Euler de $\mathcal{S}$. Logo, se escolhermos um $\mathcal{S}$ que satisfaça todas as hipóteses e que esteja inteiramente na região onde se encontram os estados de coexistência, a soma dos índices dos pontos fixos vai ser igual ao número de pontos fixos de coexistência em $\mathcal{S}$ (pois todos os índices são iguais a 1) e esse número deve ser igual à característica de Euler.

Iremos considerar as seguintes subvariedades de $\operatorname{Sim}_{M}$ :

$$
\operatorname{Sim}_{M}^{\epsilon}=\left\{\vec{\eta} \in \operatorname{Sim}_{M} \mid \eta_{\sigma} \geq \epsilon \forall \sigma\right\}, \operatorname{com} \epsilon>0 .
$$

Todas elas estão inteiramente na região dos estados de coexistência, satisfazem as hipóteses do teorema e tem característica de Euler 1, o que significa, pelo nosso raciocínio anterior que, se $\vec{F}$ aponta para fora de $\operatorname{Sim}_{M}^{\epsilon}$ ao longo de toda a sua borda, então existe exatamente um ponto fixo de coexistência em $\operatorname{Sim}_{M}^{\epsilon}$. As bordas de $\operatorname{Sim}_{M}^{\epsilon}$ são as facetas dadas por $\eta_{\sigma}=\epsilon \mathrm{e}$ $\operatorname{logo} \vec{F}$ aponta pra fora ao longo de toda a borda se e somente se $F_{\sigma}<0$ sempre que $\eta_{\sigma}=\epsilon$ e $\eta_{\sigma^{\prime}} \geq \epsilon \forall \sigma^{\prime} \neq \sigma$. Defina então

$$
p_{\text {min }}=\min _{\sigma \neq \sigma^{\prime}}\left\{p_{\sigma \rightarrow \sigma^{\prime}}\right\} \quad \text { e } \quad p_{\max }=\max _{\sigma \neq \sigma^{\prime}}\left\{p_{\sigma \rightarrow \sigma^{\prime}}\right\}
$$

${ }^{1}$ Todas as subvariedades que tomaremos satisfazem os detalhes técnicos necessários. 
Pela nossa hipótese a respeito da regra de confiança (esqueleto completo) temos $p_{\min }>0$. Se $\eta_{\sigma}=\epsilon$, então

$$
\begin{gathered}
F_{\sigma}=\epsilon^{2} \sum_{\sigma^{\prime}} \eta_{\sigma^{\prime}} p_{\sigma^{\prime} \rightarrow \sigma}-\epsilon \sum_{\sigma^{\prime}} \eta_{\sigma^{\prime}}^{2} p_{\sigma \rightarrow \sigma^{\prime}} \leq \epsilon^{2} p_{\max } \sum_{\sigma^{\prime}} \eta_{\sigma^{\prime}}-\epsilon p_{\min } \sum_{\sigma^{\prime}} \eta_{\sigma^{\prime}}^{2} \leq \\
\leq \epsilon^{2} p_{\max }-\epsilon p_{\min } \min _{\vec{\eta} \in \operatorname{Sim} M}\left\{\sum_{\sigma^{\prime}} \eta_{\sigma^{\prime}}^{2}\right\} .
\end{gathered}
$$

Usando multiplicadores de Lagrange é fácil provar que

$$
\min _{\vec{\eta} \in \operatorname{Sim}_{M}}\left\{\sum_{\sigma} \eta_{\sigma}^{q}\right\}=\frac{1}{M^{q-1}} \forall q>0 .
$$

Logo a desigualdade 4.3.3 implica que

$$
F_{\sigma} \leq \epsilon^{2} p_{\max }-\epsilon \frac{p_{\min }}{M}
$$

de forma que $F_{\sigma}<0$ se tivermos

$$
\epsilon^{2} p_{\max }-\epsilon \frac{p_{\min }}{M}<0 \Leftrightarrow \epsilon<\frac{p_{\min }}{M p_{\max }} .
$$

Então se escolhermos um $\epsilon$ obedecendo a desigualdade 4.3.6, segue que $\vec{F}$ aponta para fora de $\operatorname{Sim}_{M}^{\epsilon}$ ao longo de toda a borda e nós podemos aplicar o teorema na variedade $\operatorname{Sim}_{M}^{\epsilon}$. Isso significa que podemos aplicar o teorema para um valor de $\epsilon$ positivo e arbitrariamente próximo de 0 , de forma que só deve existir um ponto fixo de coexistência e esse ponto fixo pode ser melhor localizado usando valores cada vez maiores de $\epsilon$ (pois o "tamanho" de $\operatorname{Sim}_{M}^{\epsilon}$ diminui $\operatorname{com} \epsilon$ ). Chegamos a conclusão que, se todos os parâmetros da regra de confiança forem não nulos (o que equivale a dizer que o esqueleto direcionado é um grafo direcionado completo), então existe exatamente um ponto fixo em que todas as opiniões coexistem e as coordenadas dele obedecem

$$
\eta_{\sigma} \geq \frac{p_{\min }}{M p_{\max }}
$$

Esse resultado cobre toda a região do espaço de parâmetros em que $p_{\sigma \rightarrow \sigma^{\prime}} \neq 0 \forall \sigma \neq \sigma^{\prime}$. Porém toda regra corresponde a um ponto no espaço de parâmetros, que pode ser levado a essa região por uma perturbação infinitesimal (trocando todos os $p_{\sigma \rightarrow \sigma^{\prime}}$ que são iguais a 0 por um valor $\epsilon$ arbitrariamente pequeno). Para estudar essas perturbações, vamos utilizar principalmente o teorema da função implícita (TFI). Como o jacobiano de $\vec{F}$, avaliado em um ponto fixo de coexistência, tem todos os autovalores com parte real positiva, com a exceção do artefato de imersão (chegamos nesse resultado na seção 4.2.2), podemos usar 
o TFI para chegar à conclusão que as coordenadas do ponto fixo em $\operatorname{Sim}_{M}$ são funções contínuas dos parâmetros da regra de confiança (para satisfazer as condições do teorema bastaria fazer uma construção como a que fizemos para mostrar a irrelevância dos artefatos de imersão na seção 1.3), no sentido que uma perturbação pequena dos parâmetros, equivale a uma perturbação pequena das coordenadas do ponto fixo. Segue que, se uma regra $\mathcal{R}$ é tal que não existem pontos fixos de coexistência, uma bifurcação deve estar acontecendo. Para entender isso, vamos começar com uma regra $\mathcal{R}^{\prime}$ que tenha esqueleto completo e fazer um caminho contínuo no espaço de parâmetros, até $\mathcal{R}$, passando apenas pela região em que $p_{\sigma \rightarrow \sigma^{\prime}} \neq 0 \forall \sigma \neq \sigma^{\prime}$. Mais precisamente, vamos definir um conjunto de regras $\mathcal{R}(\tau)$, com $\tau \in[0,1]$ e parâmetros $p_{\sigma \rightarrow \sigma^{\prime}}(\tau)$. Vamos tomar $\mathcal{R}(0)=\mathcal{R}^{\prime}, \mathcal{R}(1)=\mathcal{R}$ e todos os $p_{\sigma \rightarrow \sigma^{\prime}}$ contínuos, tais que $0<p_{\sigma \rightarrow \sigma^{\prime}}(\tau) \leq 1$ se $\tau \neq 1$. Lembramos que todos os $\mathcal{R}(\tau)$ tem esqueleto completo, com a exceção de $\mathcal{R}(1)$, que é igual a $\mathcal{R}$. O nosso resultado com regras de esqueleto completo nos diz que temos exatamente um ponto fixo de coexistência para cada regra desse caminho e que esse ponto fixo é uma função contínua dos parâmetros da regra. Portanto, devemos ter associado ao caminho $\mathcal{R}(\tau)$, no espaço de parâmetros, um caminho formado pelos pontos fixos de coexistência, $\vec{\eta}^{*}(\tau)$ em $\operatorname{Sim}_{M}$, que deve ser contínuo com respeito a $\tau$, com a exceção de $\tau=1$, quando não temos um ponto fixo de coexistência. Por outro lado, o ponto $\vec{\eta}_{\lim } \equiv \lim _{\tau \rightarrow 1} \vec{\eta}^{*}(\tau)$ deve ser um ponto fixo do modelo. Por hipótese, esse não é um ponto fixo de coexistência e o nosso caminho $\vec{\eta}^{*}(\tau)$ deve estar colidindo com outro ponto fixo na borda do espaço de fase, para $\tau=1$, correspondendo a uma bifurcação.

Temos opiniões que são extintas em $\vec{\eta}_{\text {lim }}$, de forma que esse ponto fixo deve se enquadrar em algum dos casos estudados na seção 4.2.3. Para identificar esse caso, chamamos a atenção para o fato do jacobiano de $\vec{F}$ ser uma função contínua das coordenadas $\vec{\eta}$ (isso pode ser trivialmente verificado pela equação 4.2.2), de forma que o jacobiano de $\vec{F}$, avaliado no ponto fixo $\vec{\eta}^{*}(\tau)$ é uma função contínua de $\tau$. Como os coeficientes do polinômio característico de uma matriz $M$ podem ser expressos como somas de determinantes de submatrizes de $M$, então eles são funções contínuas dos elementos de $M$, de forma que os coeficientes do polinômio característico do jacobiano avaliado em $\vec{\eta}^{*}(\tau)$ são funções contínuas de $\tau$. Finalmente, as raízes de um polinômio mônico (ou seja, onde o coeficiente da ordem mais alta é 1) são uma função contínua dos seus coeficientes ${ }^{2}$, no sentido que o conjunto de raízes como um todo, contadas as multiplicidades, é contínuo. Os autovalores são as raízes

\footnotetext{
${ }^{2} \mathrm{~A}$ rigor, essa noção de continuidade é mais delicada, pois uma topologia específica está subentendida. Se simplesmente definirmos uma função $\rho: \mathbb{C}^{n} \rightarrow \mathbb{C}^{n}$, onde $\mathbb{C}^{n}$ tem a topologia usual e $\rho$ leva cada polinômio mônico a alguma permutação fixa de suas raízes, não vamos ter em geral uma função contínua. Um exemplo é uma função que leva cada polinômio de ordem $n$ a uma $n$-tupla constituída das raízes ordenadas lexicograficamente (e repetidas de acordo com a multiplicidade). O impecilho principal para se construir uma função contínua nesses moldes são os pontos de ramificação que são inevitavelmente introduzidos para $n>1$ (a raiz quadrada que surge nas raízes de um polinômio de segundo grau é o exemplo mais simples) e que impedem que uma permutação das raízes seja escolhida de forma consistente, sem abrir mão da continuidade. Por outro lado, se usarmos uma função parecida, mas definida do espaço topológico $\mathbb{C}^{n}$ no espaço $\mathbb{C}^{n} / \sim$, onde $\sim$ é uma relação de equivalência tal que $x \sim y$ se e somente se $x, y \in \mathbb{C}^{n}$ podem ser levados de um no outro por uma permutação de suas coordenadas, então a função $\rho: \mathbb{C}^{n} \rightarrow \mathbb{C}^{n} / \sim$ é única e contínua.
} 
do polinômio característico, de forma que o espectro como um todo, com os autovalores indistinguíveis entre si, é contínuo com respeito a $\tau$. Como temos opiniões que são extintas em $\vec{\eta}_{\text {lim }}$, então $\Omega \neq \varnothing$ e todos os autovalores que vem do bloco $\mathcal{J}_{\Omega}^{*}$, na equação 4.2 .4 , tem parte real menor ou igual a 0 (lembrando que o artefato de imersão vem do bloco $\mathcal{J}_{\Delta}^{*}$ ). Porém, com a exceção do artefato, todos os autovalores tem parte real positiva, para $\tau \neq 1$, de forma que o limite quando $\tau$ tende a 1, da parte real de qualquer um dos autovalores do jacobiano avaliado em $\vec{\eta}^{*}(\tau)$ é maior ou igual a 0 . Então, a única forma de conciliar a continuidade dos autovalores com respeito a $\tau$ com essas conclusões é se todos os autovalores, que vem do bloco $\mathcal{J}_{\Omega}^{*}$, no jacobiano avaliado em $\vec{\eta}_{\text {lim }}$, forem nulos, ou seja $\lambda_{\sigma}=0 \forall \sigma \in \Omega$, ou ainda $\mathcal{J}_{\Omega}^{*}=0$, já que esse bloco é diagonal. Voltando à nossa análise na seção 4.2.3, isso é equivalente a $\sigma \notin \Delta_{-}(\mathcal{R}) \forall \sigma \in \Omega$, ou seja, nenhuma das opiniões extintas em $\vec{\eta}_{\text {lim }}$ é capaz de convencer nenhuma das opiniões sobreviventes. Como $\Omega=\bar{\Delta}$, essa expressão é equivalente a $\bar{\Delta} \cap \Delta_{-}(\mathcal{R})=\varnothing$ que, por definição, significa que $\Delta$ é uma fonte em $\mathcal{R}$ (ver seção 1.2), e como $\Omega=\bar{\Delta} \neq \varnothing, \Delta$ é diferente de $V(\mathcal{R})$. Resumindo, se uma regra $\mathcal{R}$ não possui pontos fixos de coexistência, deve existir uma fonte $\Delta \neq V(\mathcal{R})$ em $\mathcal{R}$.

Por outro lado, se $\mathcal{R}$ possui apenas uma única componente e ela é fortemente conexa, então pelo teorema A.1.3, $V(\mathcal{R})$ é uma fonte minimal e logo não existe um conjunto de opiniões $\Delta$ distinto de $V(\mathcal{R})$ que seja uma fonte. Segue que $\mathcal{J}_{\Omega}^{*} \neq 0$ para todos os pontos fixos em que existem opiniões extintas, implicando que existe um ponto fixo em que todas as opiniões coexistem (pois ele não poderia colidir na borda do espaço de fase, sem violar os nossos argumentos de continuidade). Isso também implica que a unicidade do ponto fixo de coexistência não é afetada, pois as únicas bifurcações que poderiam gerar outro ponto fixo, seriam tais que o jacobiano avaliado nele teria autovalores com parte real nula, além do artefato, contrariando nossas conclusões da seção 4.2.2 (na verdade, só deixariamos de ter unicidade se $\mathcal{R}$ tivesse mais de uma componente).

Suponha por outro lado que $\mathcal{R}$ possui apenas uma única componente, mas ela não é fortemente conexa. Então o mesmo teorema A.1.3 implica que existe um $\Delta$ que é um sorvedouro minimal e esse conjunto é diferente de $V(\mathcal{R})$, mas então definindo

$$
\eta_{\Delta}=\sum_{\sigma \in \Delta} \eta_{\sigma}
$$

temos

$$
\dot{\eta}_{\Delta}=\sum_{\sigma \in \Delta} \sum_{\sigma^{\prime} \in \bar{\Delta}}\left(\eta_{\sigma}^{2} \eta_{\sigma^{\prime}} p_{\sigma^{\prime} \rightarrow \sigma}-\eta_{\sigma} \eta_{\sigma^{\prime}}^{2} p_{\sigma \rightarrow \sigma^{\prime}}\right)
$$

pois a soma em que $\sigma, \sigma^{\prime} \in \Delta$ se anula, uma vez que $\eta_{\sigma}^{2} \eta_{\sigma^{\prime}} p_{\sigma^{\prime} \rightarrow \sigma}-\eta_{\sigma} \eta_{\sigma^{\prime}}^{2} p_{\sigma \rightarrow \sigma^{\prime}}$ é antissimétrico por uma troca de $\sigma$ por $\sigma^{\prime}$. Segue que

$$
\dot{\eta}_{\Delta}=\sum_{\sigma \in \Delta}\left(\eta_{\sigma}^{2} \sum_{\sigma^{\prime} \in \bar{\Delta} \cap \Delta_{-}} \eta_{\sigma^{\prime}} p_{\sigma^{\prime} \rightarrow \sigma}-\eta_{\sigma} \sum_{\sigma^{\prime} \in \bar{\Delta} \cap \Delta_{+}} \eta_{\sigma^{\prime}}^{2} p_{\sigma \rightarrow \sigma^{\prime}}\right) .
$$


Finalmente, como $\Delta$ é um sorvedouro, $\bar{\Delta} \cap \Delta_{+}=\varnothing$. Porém, $\mathcal{R}$ é conexo (já que ele só tem uma componente), o que implica que $\Gamma_{\Delta} \cap \bar{\Delta} \neq \varnothing$. Mas $\Gamma_{\Delta}=\Delta_{+} \cup \Delta_{-}$e portanto $\Delta_{-} \cap \bar{\Delta} \neq \varnothing$. Substituindo na equação 4.3 .8 obtemos

$$
\dot{\eta}_{\Delta}=\sum_{\sigma \in \Delta} \eta_{\sigma}^{2} \sum_{\sigma^{\prime} \in \Delta_{-} \cap \bar{\Delta}} \eta_{\sigma^{\prime}} p_{\sigma^{\prime} \rightarrow \sigma}
$$

e essa expressão nunca se anula se todos os $\eta_{\sigma}$ forem não-nulos, implicando que não existem pontos fixos de coexistência. Em suma, se uma regra de confiança tem uma componente, então ela tem pontos fixos de coexistência se e somente se ela for fortemente conexa e os pontos fixos de coexistência são únicos, quando existem.

Podemos agora aplicar esses resultados para chegar no cenário em que $\mathcal{R}$ tem mais de uma componente. Para que exista um ponto fixo de coexistência, no caso em que $\mathcal{R}$ tem $k$ componentes, esses pontos fixos devem existir para as regras restritas a cada uma das componentes, de forma que se $\mathcal{R}$ for uma união de componentes fortementes conexas, existe exatamente um hiperplano, $k-1$ dimensional, constítuido de pontos fixos de coexistência (definido pela equação 4.2.13) e não existem pontos fixos de coexistência no caso contrário.

Finalmente, as trajetórias na variedade $\mathcal{M}_{\Delta}$ :

$$
\mathcal{M}_{\Delta}=\left\{\vec{\eta} \in \operatorname{Sim}_{M} \mid \eta_{\sigma}=0 \forall \sigma \in \bar{\Delta}\right\}
$$

para um modelo com regra $\mathcal{R}$, são equivalentes às de um modelo com regra $\mathcal{R}_{\Delta}$, pois se $\vec{\eta} \in \mathcal{M}_{\Delta}$, então as equações de campo médio ficam

$\dot{\eta}_{\sigma}=\eta_{\sigma} \sum_{\sigma^{\prime}} \eta_{\sigma^{\prime}}\left(\eta_{\sigma} p_{\sigma^{\prime} \rightarrow \sigma}-\eta_{\sigma^{\prime}} p_{\sigma \rightarrow \sigma^{\prime}}\right)= \begin{cases}0 & \text {, se } \sigma \notin \Delta \\ \eta_{\sigma} \sum_{\sigma^{\prime} \in \Delta} \eta_{\sigma^{\prime}}\left(\eta_{\sigma} p_{\sigma^{\prime} \rightarrow \sigma}-\eta_{\sigma^{\prime}} p_{\sigma \rightarrow \sigma^{\prime}}\right) & \text {, se } \sigma \in \Delta .\end{cases}$

Segue que existem pontos fixos em que as opiniões em $\Delta$ são as que sobrevivem se e somente se $\mathcal{R}_{\Delta}$ for a união de componentes fortemente conexas e nesse caso, se $\mathcal{R}_{\Delta}$ tiver $k$ componentes, esses pontos fixos vão estar contidos em um único hiperplano, com $k-1$ dimensões. Todos os pontos fixos do modelo se enquadram nesse último caso, de forma que agora possuímos uma descrição completa de quais são os pontos fixos do modelo.

\subsection{O modelo Sznajd com ruído}

Algumas das estruturas que surgem nos resultados das seções 4.2 e 4.3 são estruturalmente instáveis, pois exibem pontos fixos que não são isolados. A introdução de um ruído nas equações de campo médio deve, por outro lado, eliminar essas estruturas, ou torná-las estruturalmente estáveis. Iremos primeiro introduzir um ruído uniforme no modelo Sznajd usual, para depois adicionar um ruído infinitesimal no modelo com regras de confiança, discutindo o que acontece quando o ruído não é uniforme. 


\subsubsection{Modelo usual com ruído uniforme}

Vamos começar com as equações para o modelo sem ruído e com regras de confiança. Para introduzir um ruído uniforme no modelo, iremos introduzir um parâmetro $\nu$ entre 0 e 1 e mudaremos as regras do modelo da seguinte forma:

- Com probabilidade $\nu$, um sítio é escolhido aleatoriamente e muda de opinião para uma opinião escolhida, também de forma aleatória.

- Se não (probabilidade $1-\nu$ ), as regras do modelo usual são seguidas.

Segue que a equação de campo médio passa a ser

$$
\dot{\eta}_{\sigma}=(1-\nu) F_{\sigma}+\nu\left(\frac{1}{M}\left(1-\eta_{\sigma}\right)-\eta_{\sigma}\left(1-\frac{1}{M}\right)\right)
$$

e para $\nu=0$ temos o modelo sem ruído. O termo $\nu\left(1-\eta_{\sigma}\right) / M$ correponde à probabilidade de um sítio, que não tem opinião $\sigma$, passar a ter opinião $\sigma$, por causa da regra de ruído, enquanto que o termo $\nu \eta_{\sigma}(1-1 / M)$ é a probabilidade que um sítio, com opinião $\sigma$, deixe de ter opinião $\sigma$, por causa da regra de ruído. Podemos reorganizar a equação 4.4.1 para uma forma mais simples. Como,

$$
\frac{1}{M}\left(1-\eta_{\sigma}\right)-\eta_{\sigma}\left(1-\frac{1}{M}\right)=\frac{1}{M}-\frac{\eta_{\sigma}}{M}-\eta_{\sigma}+\frac{\eta_{\sigma}}{M}=\frac{1}{M}-\eta_{\sigma}=\frac{1}{M} \sum_{\sigma^{\prime}}\left(\eta_{\sigma^{\prime}}-\eta_{\sigma}\right),
$$

então a equação de campo médio se torna, a menos da escala de tempo

$$
\dot{\eta}_{\sigma}=F_{\sigma}+\theta \sum_{\sigma^{\prime}}\left(\eta_{\sigma^{\prime}}-\eta_{\sigma}\right), \text { onde } \theta=\frac{\nu}{M(1-\nu)} .
$$

Iremos chamar o parâmetro $\theta$ de intensidade do ruído. Ele tem um papel equivalente ao de $\nu$, porém ele varia entre 0 (ausência de ruído) e $\infty$ (os sítios trocam de opinião de maneira aleatória, sem interagir entre si). Para o modelo usual, a equação 4.4.2 se torna

$$
\dot{\eta}_{\sigma}=\sum_{\sigma^{\prime}}\left(\eta_{\sigma}-\eta_{\sigma^{\prime}}\right)\left(\eta_{\sigma} \eta_{\sigma^{\prime}}-\theta\right) .
$$

Iremos agora encontrar os pontos fixos do modelo e estabelecer quais são as bifurcações que acontecem, a medida que a intensidade do ruído cresce. Iremos aproveitar algumas simetrias das equações 4.4.3, para resolver explicitamente os pontos fixos e algumas de suas propriedades. Primeiramente, iremos definir $Q(\vec{\eta})$ como

$$
Q=\sum_{\sigma} \eta_{\sigma}^{2}
$$


Podemos reescrever a equação 4.4.3 usando essa função da seguinte forma:

$$
\dot{\eta}_{\sigma}=\eta_{\sigma}^{2}-(\theta M+Q) \eta_{\sigma}+\theta .
$$

O ponto importante é que podemos usar 4.4.4 para escrever uma equação para os pontos fixos. Suponha que $\vec{\eta}^{*}$ seja um ponto fixo da equação 4.4.3. Então, se definirmos $Q^{*} \equiv$ $Q\left(\vec{\eta}^{*}\right)$, segue da equação 4.4.4 que todas as coordenadas de $\vec{\eta}^{*}$ obedecem

$$
\eta_{\sigma}^{2}-\left(\theta M+Q^{*}\right) \eta_{\sigma}+\theta=0 .
$$

Por outro lado, isso significa que toda coordenada de $\vec{\eta}^{*}$ é uma solução de

$$
\eta^{2}-\left(\theta M+Q^{*}\right) \eta+\theta=0 .
$$

O principal desafio de tentar encontrar os pontos fixos através da equação 4.4 .5 é que suas soluções dependem de $Q^{*}$, que por sua vez depende das soluções. Porém, fixando um valor para $Q^{*}$, a equação 4.4.6 possui no máximo 2 soluções. Apesar de nem todos os valores de $Q^{*}$ corresponderem a algum ponto fixo, mesmo assim, isso significa que as coordenadas de um ponto fixo só podem ter no máximo 2 valores distintos. Em outras palavras, todos os pontos fixos de 4.4.3 são tais que existem 2 inteiros positivos $m$ e $n$, tais que $\vec{\eta}^{*}$ possui $m$ coordenadas iguais a $\eta_{+}, n$ coordenadas iguais a $\eta_{-}$e $m+n=M$. A principal vantagem de reescrever o problema dessa forma, é que agora só temos 2 variáveis (uma das quais pode ser eliminada impondo que a soma das coordenadas é 1 , ou seja $m \eta_{+}+n \eta_{-}=1$ ) e os pontos fixos são parametrizados por um par de números inteiros. Além disso, se trocarmos $M$ por $m+n$ e não fixarmos o valor da soma de $m \operatorname{com} n$, estaremos "resolvendo ao mesmo tempo", todos os valores possíveis de $M$.

Voltando na equação 4.4 .3 e igualando a derivada a 0 , vem

$$
\left\{\begin{array}{l}
m \eta_{+}+n \eta_{-}=1 \\
\left(\eta_{+}-\eta_{-}\right)\left(\eta_{+} \eta_{-}-\theta\right)=0
\end{array}\right.
$$

pois o termo $\left(\eta_{\sigma}-\eta_{\sigma^{\prime}}\right)$ da equação 4.4.3 só é não nulo se $\eta_{\sigma}=\eta_{+}$e $\eta_{\sigma^{\prime}}=\eta_{-}$(ou vice-versa), de forma que todos os termos da soma em $\sigma^{\prime}$ que não são nulos, são iguais entre si.

Uma solução trivial é $\eta_{+}=\eta_{-}$, que dá $\eta_{\sigma}=1 / M \forall \sigma$. Dividindo por $\left(\eta_{+}-\eta_{-}\right)$, então $\eta_{+} \eta_{-}=\theta$ e substituindo a primeira equação de 4.4.7, vem $m \eta_{+}^{2}-\eta_{+}+n \theta=0$. Supondo sem perda de generalidade que $m \eta_{+} \geq n \eta_{-}$, então

$$
\eta_{+}(m, n)=\frac{1+\sqrt{1-4 m n \theta}}{2 m} \quad \text { e } \quad \eta_{-}(m, n)=\frac{1-\sqrt{1-4 m n \theta}}{2 n},
$$

e iremos identificar esses pontos fixos como sendo do tipo $(m, n)$. Note que a expressão de $\eta_{+}$não faz sentido e $\eta_{-}=0$, quando $m=0$ e a expressão para $\eta_{-}$é mal definida para $n=0$, porém isso não tem relevância, pois a nossa imposição $m \eta_{+} \geq n \eta_{-}$, implica que as equações 4.4.8 supõem que se todas as coordenadas são iguais então $n=0$, ao invés de $m=0$ e se 
$n=0$, o valor de $\eta_{-}$não é importante (já que o valor de $\eta_{+}=1 / m$ é consistente). Por essa mesma razão, iremos identificar por $(M, 0)$ o ponto fixo proveniente da solução trivial $\eta_{+}=\eta_{-}$.

As bifurcações desse modelo são então todas devido a colisões entre pontos fixos. Uma bifurcação trivial acontece quando $\eta_{+}=\eta_{-}$é uma solução de $\eta_{+} \eta_{-}=\theta$, o que acontece para $\theta_{0}=1 / M^{2}$. A equação 4.4.8 implica que todos os pontos fixos tais que $m \geq n$ colidem no centro do simplexo para esse valor de $\theta$. Da definição de $\theta$, segue que essa bifurcação acontece quando a probabilidade de seguir a regra de ruído é $\nu_{0}=1 /(M+1)$. A outra bifurcação acontece quando os pontos fixos do tipo $(m, n)$ colidem com os do tipo $(n, m)$. Nessa bifurcação, os pontos fixos se aniquilam e portanto a colisão acontece para um valor de $\theta$ tal que as raízes na equação 4.4.8 passem a ser complexas, ou seja

$$
\theta_{m, n}=\frac{1}{4 m n} \Rightarrow \nu_{m, n}=\frac{M}{M+4 m n}=\frac{1}{1+\frac{4 m n}{M}} .
$$

Como

$$
(m-n)^{2} \geq 0 \Rightarrow(m+n)^{2}-4 m n \geq 0 \Rightarrow M \geq \frac{4 m n}{M},
$$

então $\nu_{0} \leq \nu_{m, n}$ e a igualdade só vale se $m=n$. Portanto se a probabilidade de seguir o ruído for maior do que $\nu_{1, M-1}=M /(5 M-4) \leq 1 / 3$, o único ponto fixo do modelo é o ponto em que todas as opiniões aparecem na mesma proporção na rede.

\subsubsection{O modelo com regras de confiança e ruído infinitesimal}

Com a introdução de regras de confiança, o tratamento simplificado da seção 4.4.1 não é mais possível. Além disso, para uma intensidade de ruído arbitrária, um tratamento nos moldes das seções 4.2 e 4.3 também não é possível. Logo, iremos nos restringir a um ruído com intensidade infinitesimal e fazer um tratamento perturbativo, partindo dos resultados obtidos na ausência de ruído. Como vamos introduzir um ruído infinitesimal, é de se esperar que algumas estruturas saiam inalteradas. Por exemplo, podemos usar o teorema da função implícita para chegar a conclusão que, se $\mathcal{J}_{\vec{F}}^{*}$, o jacobiano avaliado em algum ponto fixo, tiver todos os autovalores não nulos, com a exceção do artefato de imersão, causado pela conservação da soma das componentes, então esse ponto fixo sobrevive a perturbações do sistema que também sejam tais que a soma das coordenadas é conservada (como a introdução de um ruído), ou seja, eles serão estruturalmente estáveis. Nossos resultados anteriores implicam que isso quer dizer que apenas dois tipos de pontos fixos serão ameaçados pela introdução do ruído:

- Pontos fixos tais que o grafo $\mathcal{R}_{\Delta}$, obtido a partir da regra de confiança $\mathcal{R}$ e do conjunto de opiniões que sobrevivem nesse ponto fixo, $\Delta$, tem mais de uma componente. 
- Pontos fixos em que existe alguma opinião $\sigma$ que é uma opinião extinta $(\sigma \in \Omega)$ e tal que $\lambda_{\sigma}=0$.

Além disso, no caso de pontos fixos em que opiniões são extintas, mesmo que eles não se enquadrem em nenhum desses dois casos, precisaremos verificar se os pontos permanecem dentro do espaço de fase $\operatorname{Sim}_{M}$. Vamos fazer essa análise supondo que tenhamos um pontto fixo do modelo com ruído de intensidade $\theta$, onde $\theta$ é pequeno, e tomando o limite $\theta \rightarrow 0$, para verificar quando esse limite dá um ponto fixo com determinadas propriedades. Para isso, faremos uma expansão em Taylor, desprezando termos de ordem $\theta^{2}$, para chegar em uma equação para um vetor que, grosso modo, se comporta como uma derivada do ponto fixo, com respeito a $\theta$.

O que vamos obter ao final dessa seção é um resultado parcial sobre o que acontece com a introdução do ruído. Os resultados obtidos nessa seção são comparativamente mais fracos, já que, como é de se esperar, existem várias dificuldades em tentar um tratamento desse tipo, principalmente porque só conseguimos informações qualitativas a respeito dos pontos fixos, nas seções anteriores. Apesar disso, conseguimos mostrar que o comportamento do modelo com ruído, mesmo de intensidade infinitesimal, é muito mais rico que o do modelo sem ruído, com o surgimento de atratores que não são estáticos, por exemplo.

Vamos começar expandindo a equação 4.4.2, que descreve o caso geral com ruído uniforme:

$$
\dot{\eta}_{\sigma}=\sum_{\sigma^{\prime}}\left(\eta_{\sigma} \eta_{\sigma^{\prime}}\left(\eta_{\sigma} p_{\sigma^{\prime} \rightarrow \sigma}-\eta_{\sigma^{\prime}} p_{\sigma \rightarrow \sigma^{\prime}}\right)+\theta\left(\eta_{\sigma^{\prime}}-\eta_{\sigma}\right)\right)
$$

Podemos considerar um ruído mais geral em que, com probabilidade $1-\nu$, as regras do modelo usual são seguidas e, com probabilidade $\nu$, escolhemos um sítio aleatoriamente. Se esse sítio escolhido tiver opinião $\sigma$, então, com probabilidade $n_{\sigma \rightarrow \sigma^{\prime}}$, ele passa a ter opinião $\sigma^{\prime}$, o que nos leva à equação (definindo $\theta$ da maneira usual, segundo a equação 4.4.2)

$$
\dot{\eta}_{\sigma}=\sum_{\sigma^{\prime}}\left(\eta_{\sigma} \eta_{\sigma^{\prime}}\left(\eta_{\sigma} p_{\sigma^{\prime} \rightarrow \sigma}-\eta_{\sigma^{\prime}} p_{\sigma \rightarrow \sigma^{\prime}}\right)+\theta\left(\eta_{\sigma^{\prime}} n_{\sigma^{\prime} \rightarrow \sigma}-\eta_{\sigma} n_{\sigma \rightarrow \sigma^{\prime}}\right)\right) .
$$

Finalmente, podemos simplesmente escolher um campo contínuo e suficientemente diferenciável $\vec{G}$, definido em $\operatorname{Sim}_{M}$, tal que $\overrightarrow{1} \cdot \vec{G}=0$ (para conservar a soma das variáveis $\eta$ durante a evolução temporal) e $G_{\sigma}>0$ para todos os pontos tais que $\eta_{\sigma}=0$, para todo $\sigma$. Essa última restrição impede que opiniões sejam extintas e garante que as variáveis serão sempre positivas ao longo de toda a evolução temporal. Como veremos mais adiante, ela também é responsável por manter dentro do espaço de fase os pontos fixos em que opiniões são extintas e que sobrevivem à adição de ruído. Isso resulta na equação:

$$
\dot{\eta}_{\sigma}=F_{\sigma}(\vec{\eta})+\theta G_{\sigma}(\vec{\eta}) .
$$

Se supusermos que temos funções, $\widetilde{\eta}(\theta)$, descrevendo cada um dos pontos fixos do modelo com ruído, como uma função contínua de $\theta$, podemos perguntar quais dos pontos fixos 
do modelo sem ruído são acessíveis, após tomarmos o limite $\theta \rightarrow 0$ dessas funções. Essa pergunta é relevante, pois se tivermos, por exemplo, um conjunto independente, $\Delta$, na regra de confiança, é de se esperar que a adição de ruído, não importa o quão pequeno, faça com que $\mathcal{M}_{\Delta}$ deixe de ser um conjunto de pontos fixos (ou seja, deve ocorrer uma bifurcação). Podemos ver isso no exemplo do modelo com ruído uniforme restrito a essas opiniões (somente com a regra de confiança $\mathcal{R}_{\Delta}$ ), que só tem um ponto fixo (que é atrativo) no interior do espaço de fase. A conclusão é que nem todos os pontos fixos do modelo sem ruído devem ser acessíveis a partir do modelo com ruído. Além disso, como o ruído seria a única forma de "comunicação" das opiniões em $\Delta$ com as em $\bar{\Delta}$, os pontos fixos que são acessíveis devem depender da forma do ruído.

Por outro lado, se um ponto fixo (do modelo sem ruído) for acessível, como o limite de um ponto fixo do modelo com ruído quando $\theta \rightarrow 0$, isso significa que ele sobrevive à adição de ruído, pois temos um ponto fixo arbitrariamente próximo dele quando usamos um ruído arbitrariamente pequeno (caso contrário, ele não seria o limite). Por essa razão, iremos chamar esses pontos fixos de robustos.

Como já argumentamos, os pontos fixos robustos devem em geral depender da forma do ruído que estamos utilizando, com a exceção dos pontos fixos estruturalmente estáveis (quando o único autovalor nulo do jacobiano $\mathcal{J}_{\vec{F}}^{*}$, avaliado no ponto fixo, for o artefato de imersão), que serão sempre robustos. Para encontrar quais são os pontos fixos robustos, iremos partir da hipótese que podemos descrever os pontos fixos por funções $\widetilde{\eta}(\theta)$, que são, não só contínuas, como diferenciáveis, com respeito a $\theta^{3}$, de forma que $\widetilde{\eta}$ tem uma derivada $\vec{\psi}=\partial \widetilde{\eta} / \partial \theta$, contínua com respeito a $\theta$. Vamos começar com a seguinte equação, que é uma consequência da definição de $\widetilde{\eta}$ e da equação de campo médio, com ruído de intensidade $\theta$ :

$$
\vec{F}(\widetilde{\eta}(\theta))+\theta \vec{G}(\widetilde{\eta}(\theta))=\overrightarrow{0} .
$$

A equação 4.4.12, para um ruído de intensidade $\alpha \theta$ se torna

$$
\vec{F}(\widetilde{\eta}(\alpha \theta))+\alpha \theta \vec{G}(\widetilde{\eta}(\alpha \theta))=\overrightarrow{0} .
$$

Escolhendo um valor $\alpha$ tal que $0<\alpha<1$, podemos combinar as equações 4.4.12 e 4.4.13 de uma maneira que podemos fazer uma expansão de Taylor e encontrar uma equação para $\vec{\psi}$. Primeiro notamos que a equação 4.4 .13 pode ser reescrita como

$$
\overrightarrow{0}=\vec{F}(\widetilde{\eta}(\alpha \theta))+\alpha \theta \vec{G}(\widetilde{\eta}(\alpha \theta))=\vec{F}(\widetilde{\eta}(\theta+(\alpha-1) \theta))+\alpha \theta \vec{G}(\widetilde{\eta}(\theta+(\alpha-1) \theta)) .
$$

Então, se expandirmos $\widetilde{\eta}$ até termos proporcionais a $\theta$ temos

$$
\vec{F}\left(\widetilde{\eta}(\theta)+(\alpha-1) \theta \vec{\psi}(\theta)+\mathcal{O}\left(\theta^{2}\right)\right)+\alpha \theta \vec{G}\left(\widetilde{\eta}(\theta)+(\alpha-1) \theta \vec{\psi}(\theta)+\mathcal{O}\left(\theta^{2}\right)\right)=\overrightarrow{0} .
$$

\footnotetext{
${ }^{3}$ Isso é garantido se o jacobiano, $\mathcal{J}_{\vec{F}+\theta \vec{G}}$, avaliado em $\widetilde{\eta}(\theta)$ tiver apenas o artefato de imersão, como autovalor nulo.
} 
Em seguida, expandimos $\vec{F}$ e $\vec{G}$ até essa ordem, para obter

$$
\vec{F}(\widetilde{\eta}(\theta))+(\alpha-1) \theta \mathcal{J}_{\vec{F}}(\widetilde{\eta}(\theta)) \vec{\psi}(\theta)+\alpha \theta \vec{G}(\widetilde{\eta}(\theta))=\mathcal{O}\left(\theta^{2}\right)
$$

e substituindo a equação 4.4 .12 vem

$$
(\alpha-1) \theta\left(\mathcal{J}_{\vec{F}}(\widetilde{\eta}(\theta)) \vec{\psi}(\theta)+\vec{G}(\widetilde{\eta}(\theta))\right)=\mathcal{O}\left(\theta^{2}\right)
$$

e dividindo por $(\alpha-1) \theta$, chegamos finalmente em

$$
\mathcal{J}_{\vec{F}}(\widetilde{\eta}(\theta)) \vec{\psi}(\theta)+\vec{G}(\widetilde{\eta}(\theta))=\mathcal{O}(\theta) .
$$

Dessa forma, se considerarmos

$$
\vec{\psi}=\lim _{\theta \rightarrow 0} \vec{\psi}(\theta), \quad \mathcal{J}_{\vec{F}}^{*}=\lim _{\theta \rightarrow 0} \mathcal{J}_{\vec{F}}(\theta), \quad \vec{G}^{*}=\lim _{\theta \rightarrow 0} \vec{G}(\theta)
$$

no limite $\theta \rightarrow 0$, temos a equação

$$
\mathcal{J}_{\vec{F}}^{*} \cdot \vec{\psi}=-\vec{G}^{*} .
$$

Logo, se $\vec{\eta}^{*}$ for um ponto fixo robusto, devemos ter um $\widetilde{\eta}(\theta)$, tal que

$$
\vec{\eta}^{*}=\lim _{\theta \rightarrow 0} \widetilde{\eta}(\theta)
$$

de forma que deve existir uma solução para $\mathcal{J}_{\vec{F}}^{*} \cdot \vec{\psi}=-\vec{G}^{*}$. Note que, dado um $\vec{\eta}^{*}$, as variáveis que precisamos resolver são as coordenadas de $\vec{\psi}$. E portanto, precisamos impor explicitamente a condição $\overrightarrow{1} \cdot \vec{\psi}=0$ (a derivada de $\widetilde{\eta}$ deve obedecer isso, pois a soma das coordenadas de $\widetilde{\eta}$ deve ser 1 em todo o espaço de fase, ou seja, $\overrightarrow{1} . \widetilde{\eta}=1$ ).

Mais uma vez, vamos denotar por $\Omega$ o conjunto das opiniões que são extintas em $\vec{\eta}^{*}$ e por $\Delta$ o conjunto das que sobrevivem. Chamaremos de $\Delta_{1}, \ldots, \Delta_{k}$ as componentes de $\mathcal{R}_{\Delta}$ e iremos denotar as matrizes e vetores em blocos (a menos que explicitemos o contrário), com as primeiras linhas e colunas correspondendo às opiniões de $\Delta_{1}$, seguidas pelas correspondentes às opiniões em $\Delta_{2}$ e assim por diante, até as opiniões em $\Delta_{k}$, seguidas pelas opiniões em $\Omega$, quando $\Omega$ for não-nulo. Iremos também usar as notações $\vec{x}_{i}, \vec{x}_{\Delta}$ e $\vec{x}_{\Omega}$, para as coordenadas provenientes de $\Delta_{i}, \Delta$ e $\Omega$, de um vetor $\vec{x}$ e usaremos superscritos $\vec{x}^{(i)}$, para vetores que não obedecerem essa convenção. Em algumas situações, mais adiante, também teremos vetores distintos mas que tem papéis semelhantes e iremos distinguí-los ora usando uma flecha $(\rightarrow)$, ora usando um til $(\sim)$. Logo

$$
\vec{\psi}=\left[\begin{array}{c}
\vec{\psi}_{1} \\
\vdots \\
\vec{\psi}_{k} \\
\vec{\psi}_{\Omega}
\end{array}\right] \quad \text { e } \quad \vec{G}^{*}=\vec{G}\left(\vec{\eta}^{*}\right)=\left[\begin{array}{c}
\vec{G}_{1}^{*} \\
\vdots \\
\vec{G}_{k}^{*} \\
\vec{G}_{\Omega}^{*}
\end{array}\right]
$$


e o jacobiano $\mathcal{J}_{\vec{F}}^{*}$ passa a ser denotado

$$
\mathcal{J}_{\vec{F}}^{*}=\left[\begin{array}{ccccc}
\mathcal{J}_{\Delta_{1}}^{*} & 0 & \ldots & 0 & \mathcal{N}_{1} \\
0 & \mathcal{J}_{\Delta_{2}}^{*} & \ldots & 0 & \mathcal{N}_{2} \\
\vdots & \vdots & \ddots & \vdots & \vdots \\
0 & 0 & \ldots & \mathcal{J}_{\Delta_{k}}^{*} & \mathcal{N}_{k} \\
0 & 0 & \ldots & 0 & \mathcal{J}_{\Omega}^{*}
\end{array}\right],
$$

onde

$$
\left[\begin{array}{c}
\mathcal{N}_{1} \\
\vdots \\
\mathcal{N}_{k}
\end{array}\right]
$$

é a matriz $\mathcal{N}$, que aparece na equação 4.2 .4 e que foi desprezada na nossa análise das seções anteriores. Então a equação $\mathcal{J}_{\vec{F}}^{*} \cdot \vec{\psi}=-\vec{G}^{*}$, junto com a imposição $\overrightarrow{1} \cdot \vec{\psi}=0$, pode ser reescrita como

$$
\left\{\begin{array}{l}
\mathcal{J}_{\Omega}^{*} \cdot \vec{\psi}_{\Omega}=-\vec{G}_{\Omega}^{*} \\
\mathcal{J}_{\Delta_{i}}^{*} \cdot \vec{\psi}_{i}+\mathcal{N}_{i} \cdot \vec{\psi}_{\Omega}=-\vec{G}_{i}^{*} \\
\overrightarrow{1} \cdot \vec{\psi}=0
\end{array}\right.
$$

no caso $\Omega \neq \varnothing$ e como

$$
\left\{\begin{array}{l}
\mathcal{J}_{\Delta_{i}}^{*} \cdot \vec{\psi}_{i}=-\vec{G}_{i}^{*} \\
\overrightarrow{1} \cdot \vec{\psi}=0
\end{array}\right.
$$

se $\Omega=\varnothing$.

Vamos começar a análise com o caso $\Omega \neq \varnothing$. Lembramos que a matriz $\mathcal{J}_{\Omega}^{*}$ é sempre diagonal, com os termos da diagonal iguais a $\lambda_{\sigma}$ e portanto essa equação pode ser reescrita como $\psi_{\sigma} \lambda_{\sigma}=-G_{\sigma}^{*} \forall \sigma \in \Omega$. Por causa de nossas hipóteses (em particular, $G_{\sigma}>0$ sempre que $\eta_{\sigma}=0$ ), todas as coordenadas de $\vec{G}_{\Omega}^{*}$ são positivas, de forma que existe uma solução para $\vec{\psi}_{\Omega}$ se e somente se $\lambda_{\sigma} \neq 0 \forall \sigma \in \Omega$, ou de forma equivalente, se $\mathcal{J}_{\Omega}^{*}$ for inversível. Nesse caso,

$$
\psi_{\sigma}=-\frac{G_{\sigma}^{*}}{\lambda_{\sigma}}>0 \forall \sigma \in \Omega,
$$

de forma que o ponto fixo após a introdução do ruído está dentro do espaço de fase (graças a hipótese que $G_{\sigma}>0$, sempre que $\eta_{\sigma}=0$ ). Por outro lado, quando um dos $\lambda_{\sigma}$ é nulo, então a nossa hipótese de diferenciabilidade da função $\widetilde{\eta}$ falha e precisamos olhar de outra forma o que está acontecendo. Se $\sigma \in \Omega$ e $\lambda_{\sigma}=0$, então a equação 4.2.22 passa a ser, com a adição de ruído 


$$
\dot{\eta}_{\sigma} \simeq \mu_{\sigma} \eta_{\sigma}^{2}+\theta G_{\sigma}\left(\vec{\eta}^{*}\right) .
$$

Mas então, a nossa hipótese que $G_{\sigma}>0$ quando $\eta_{\sigma}=0$ implica que 4.4 .20 não se anula na vizinhança de $\mathcal{M}_{\Delta}$ (a variedade em que as opiniões em $\Delta$ sobrevivem), para nenhum $\theta>0$. Isso significa que a adição de um ruído infinitesimal "destroi" todos os pontos fixos que estiverem na variedade $\mathcal{M}_{\Delta}$ (o $\vec{\eta}^{*}$ incluso), no sentido que não existe nenhum ponto fixo do modelo com ruído que esteja arbitrariamente próximo deles e logo não existem pontos fixos do modelo com ruído, que no limite sem ruído sejam pontos fixos em que as opiniões em $\Delta$ sejam as que sobrevivem. Isso permite determinar que alguns tipos de pontos fixos que encontramos no modelo sem ruído, nunca serão robustos, não importa a forma exata do ruído. A saber, todos os pontos fixos em que opiniões são extintas e nos quais a variedade $\mathcal{M}_{\Delta}$ não seja atrativa, não são robustos.

Voltando ao caso em que temos uma solução para $\vec{\psi}_{\Omega}$, precisamos tentar agora encontrar uma solução para $\vec{\psi}_{i}$. Mais precisamente, como temos $k$ componentes; $\Delta_{1}, \ldots, \Delta_{k}$ em $\mathcal{R}_{\Delta}$ e um ponto fixo do modelo sem ruído em que as opiniões em $\Delta$ sobrevivem (por hipótese), então os resultados da seção 4.2.1 implicam que deve existir um hiperplano $\mathcal{F}$ de pontos fixos satisfazendo essas condições (ponto fixo do modelo sem ruído, com as componentes que estamos considerando) e que tem $k-1$ dimensões. Esse hiperplano é formado pelas combinações convexas de $k$ pontos fixos, que estão nas variedades $\mathcal{M}_{\Delta_{1}}, \ldots, \mathcal{M}_{\Delta_{k}}$ e que correspondem aos únicos pontos fixos de coexistência do modelo sem ruído e regras $\mathcal{R}_{\Delta_{1}}, \ldots, \mathcal{R}_{\Delta_{k}}$ respectivamente. Usando a notação da seção 4.2.1, o que iremos fazer é determinar os valores $\eta_{\Delta_{1}}^{*}, \ldots, \eta_{\Delta_{k}}^{*}$ (que parametrizam $\mathcal{F}$ ) para os quais existem soluções para os $\vec{\psi}_{i}$. Substituindo a solução $\vec{\psi}_{\Omega}=-\left(\mathcal{J}_{\Omega}^{*}\right)^{-1} \cdot \vec{G}_{\Omega}^{*}$ nas equações 4.4 .17 temos

$$
\left\{\begin{array}{l}
\mathcal{J}_{\Delta_{i}}^{*} \cdot \vec{\psi}_{i}=\mathcal{N}_{i} \cdot\left(\mathcal{J}_{\Omega}^{*}\right)^{-1} \cdot \vec{G}_{\Omega}^{*}-\vec{G}_{i}^{*} \\
\overrightarrow{1} \cdot \vec{\psi}=0 .
\end{array}\right.
$$

A forma dessas equações é parecida com as de 4.4.18, de forma que em grande parte do que se segue podemos resolver o caso $\Omega=\varnothing$ trocando $\mathcal{N}_{i} \cdot\left(\mathcal{J}_{\Omega}^{*}\right)^{-1} \cdot \vec{G}_{\Omega}^{*}$ por $\overrightarrow{0}_{i}$. Notamos que 4.4.21 especifica que o ponto fixo do modelo com ruído deve fazer parte de $\operatorname{Sim}_{M}(\overrightarrow{1} \cdot \vec{\psi}=0)$ e que cada $\vec{\psi}_{i}$ é uma solução do sistema

$$
\mathcal{J}_{\Delta_{i}}^{*} \cdot \vec{\psi}_{i}=\mathcal{N}_{i} \cdot\left(\mathcal{J}_{\Omega}^{*}\right)^{-1} \cdot \vec{G}_{\Omega}^{*}-\vec{G}_{i}^{*},
$$

que para um $\vec{\eta}^{*}$ fixo é um sistema linear, em que $\vec{\psi}_{i}$ é a incógnita. Aqui temos uma complicação, que será o nosso ponto de partida para determinar quais são os valores de $\eta_{\Delta_{i}}^{*}$ correspondentes a pontos robustos. O problema é que esse sistema possui $\left|\Delta_{i}\right|$ variáveis (as coordenadas de $\vec{\psi}_{i}$ ) e $\left|\Delta_{i}\right|$ equações (uma para coordenada, correspondente a uma opinião em $\Delta_{i}$ ). Porém, como $\mathcal{J}_{\Delta_{i}}^{*}$ é o jacobiano avaliado em um ponto fixo de coexistência, para o modelo sem ruído e a regra $\mathcal{R}_{\Delta_{i}}$ possui apenas uma componente (por definição), os resultados da seção 4.2.2 implicam que o posto dessa matriz é $\left|\Delta_{i}\right|-1$, por causa do artefato de 
imersão, de forma que o sistema é solúvel somente se as equações não forem linearmente independentes. Para determinar quando isso acontece, podemos aproveitar que o autovetor à esquerda de $\mathcal{J}_{\Delta_{i}}^{*}$ é $\overrightarrow{1}_{i}$, ou seja, $\overrightarrow{1}_{i} \cdot \mathcal{J}_{\Delta_{i}}^{*}=\overrightarrow{0}_{i}$, para chegar em

$$
\begin{gathered}
\overrightarrow{1}_{i} \cdot \mathcal{J}_{\Delta_{i}}^{*} \cdot \vec{\psi}_{i}=\overrightarrow{1}_{i} \cdot \mathcal{N}_{i} \cdot\left(\mathcal{J}_{\Omega}^{*}\right)^{-1} \cdot \vec{G}_{\Omega}^{*}-\overrightarrow{1}_{i} \cdot \vec{G}_{i}^{*} \Rightarrow \\
\overrightarrow{1}_{i} \cdot\left(-\mathcal{N}_{i} \cdot\left(\mathcal{J}_{\Omega}^{*}\right)^{-1} \cdot \vec{G}_{\Omega}^{*}+\vec{G}_{i}^{*}\right)=0 .
\end{gathered}
$$

Iremos chamar a equação 4.4.22 de equação de robustez. Para exemplificar como passar desse caso $(\Omega \neq \varnothing)$ para o caso $\Omega=\varnothing$, notamos que podemos fazer o mesmo raciocínio para a equação 4.4.18, para chegar em $\overrightarrow{1}_{i} \cdot \vec{G}_{i}^{*}=0$, que é a equação de robustez 4.4.22, após trocarmos $\mathcal{N}_{i} \cdot\left(\mathcal{J}_{\Omega}^{*}\right)^{-1}$. $\vec{G}_{\Omega}^{*}$ por $\overrightarrow{0}_{i}$. Por enquanto, não nos preocuparemos com o valor de $\overrightarrow{1} \cdot \vec{\psi}$.

Notamos agora que as equações 4.4.22, para os diferentes $i$, não são independentes. Somando sobre todas elas vem

$$
-\left(\sum_{i=1}^{k} \overrightarrow{1}_{i} \cdot \mathcal{N}_{i}\right) \cdot\left(\mathcal{J}_{\Omega}^{*}\right)^{-1} \cdot \vec{G}_{\Omega}^{*}+\sum_{i=1}^{k} \overrightarrow{1}_{i} \cdot \vec{G}_{i}^{*}=0 .
$$

Como $\overrightarrow{1} \cdot \mathcal{J}_{\vec{F}}^{*}=\overrightarrow{0}$, então segue da equação 4.4.16 que

$$
\sum_{i=1}^{k} \overrightarrow{1}_{i} \cdot \mathcal{N}_{i}=-\overrightarrow{1}_{\Omega} \cdot \mathcal{J}_{\Omega}^{*}
$$

e como $\overrightarrow{1} \cdot \vec{G}=0$, também temos

$$
\sum_{i=1}^{k} \overrightarrow{1}_{i} \cdot \vec{G}_{i}=-\overrightarrow{1}_{\Omega} \cdot \vec{G}_{\Omega}
$$

de forma que a soma de 4.4.22, para todos os $i$ dá

$$
\overrightarrow{1}_{\Omega} \cdot \mathcal{J}_{\Omega}^{*} \cdot\left(\mathcal{J}_{\Omega}^{*}\right)^{-1} \cdot \vec{G}_{\Omega}^{*}-\overrightarrow{1}_{\Omega} \cdot \vec{G}_{\Omega}^{*}=0 \Rightarrow 0=0 .
$$

Para resolver as equações 4.4.21 e encontrar os $\vec{\psi}_{i}$, é necessário que as equações 4.4.22 sejam satisfeitas. Iremos agora mostrar que se temos uma solução para 4.4.22, então sempre podemos encontrar soluções para 4.4.21. Iremos abordar as equações usando os $\eta_{\Delta_{i}}^{*}$ como variáveis. Temos $k$ equações, só que como verificamos, no máximo $k-1$ delas são independentes, de forma que podemos introduzir a equação $\overrightarrow{1} \cdot \vec{\eta}=1 \mathrm{sem}$ problemas. Introduzindo esse vínculo, temos o sistema

$$
\left\{\begin{array}{l}
\overrightarrow{1}_{i} \cdot\left(\vec{G}_{i}^{*}-\mathcal{N}_{i} \cdot\left(\mathcal{J}_{\Omega}^{*}\right)^{-1} \cdot \vec{G}_{\Omega}^{*}\right)=0 \\
\sum_{i} \eta_{\Delta_{i}}^{*}=1 .
\end{array}\right.
$$

Note que estamos usando como parâmetros nessas equações as coordenadas dos pontos de coexistência para as regras $\mathcal{R}_{\Delta_{i}}$ e que, para que uma solução corresponda a um ponto fixo 
em $\operatorname{Sim}_{M}$, é necessário que ela obedeça às desigualdades $\eta_{\Delta_{i}}^{*} \geq 0 \forall i$. Se tivermos uma solução para o sistema 4.4.23, então o sistema linear

$$
\mathcal{J}_{\Delta_{i}}^{*} \cdot \vec{\psi}_{i}=\mathcal{N}_{i} \cdot\left(\mathcal{J}_{\Omega}^{*}\right)^{-1} \cdot \vec{G}_{\Omega}^{*}-\vec{G}_{i}^{*},
$$

não é linearmente independente e como $\mathcal{J}_{\Delta_{i}}^{*}$ tem posto $\left|\Delta_{i}\right|-1$, existe uma equação desse sistema que, se retirada, torna as equações restantes linearmente independentes. Note que só precisamos analisar o caso em que esses sistemas tem mais de uma equação, já que o caso com uma equação segue automaticamente da equação 4.4.23 (o sistema só possui uma equação que é $0=0$ ). Seja então $\mathcal{J}_{i}^{\prime}$ uma submatriz de $\mathcal{J}_{\Delta_{i}}^{*}$, quadrada de ordem $\left|\Delta_{i}\right|-1 \mathrm{e}$ posto $\left|\Delta_{i}\right|-1$, obtida retirando a linha $u$ e a coluna $v ; \psi_{i}^{\prime}$, o vetor obtido após retirarmos a coordenada $v$ de $\vec{\psi}_{i} ; \mathcal{N}_{i}^{\prime}$, a matriz obtida após retirar a linha $u$ de $\mathcal{N}_{i}$ e $G_{i}^{\prime}$, o vetor obtido após retirar a coordenada $u$ de $\vec{G}_{i}^{*}$. Então o sistema de equações

$$
\mathcal{J}_{i}^{\prime} \cdot \psi_{i}^{\prime}=\mathcal{N}_{i}^{\prime} \cdot\left(\mathcal{J}_{\Omega}^{*}\right)^{-1} \cdot \vec{G}_{\Omega}^{*}-G_{i}^{\prime}
$$

é linearmente independente (pois $\operatorname{det}\left(\mathcal{J}_{i}^{\prime}\right) \neq 0$ ). Logo, se $\widetilde{\psi}_{i}$ for um vetor com coordenada $v$ igual a 0 e que é igual a

$$
\left(\mathcal{J}_{i}^{\prime}\right)^{-1} \cdot\left(\mathcal{N}_{i}^{\prime} \cdot\left(\mathcal{J}_{\Omega}^{*}\right)^{-1} \cdot \vec{G}_{\Omega}^{*}-G_{i}^{\prime}\right),
$$

após removermos a sua coordenada $v$, então $\widetilde{\psi}_{i}$ resolve o sistema 4.4 .24 (por causa da equação 4.4.23 e da definição dele), de forma que $\widetilde{\psi}_{i}+\alpha_{i} \vec{\eta}_{i}^{*}$ é a solução geral do sistema 4.4.24, pois $\mathcal{J}_{\Delta_{i}}^{*} \cdot \vec{\eta}_{i}^{*}=\overrightarrow{0}_{i}{ }^{4}$. Falta apenas então satisfazer a equação $\overrightarrow{1} \cdot \vec{\psi}=0$, o que é trivial, pois pelo menos um dos $\eta_{\Delta_{i}}^{*}$ é não-nulo e

$$
\overrightarrow{1} \cdot \vec{\psi}=\overrightarrow{1}_{\Omega} \cdot \vec{\psi}_{\Omega}+\sum_{i=1}^{k} \overrightarrow{1}_{i} \cdot \widetilde{\psi}_{i}+\sum_{i=1}^{k} \alpha_{i} \eta_{\Delta_{i}}^{*}=0 .
$$

Isso define apenas um dos $\alpha_{i}$ e portanto o conjunto de todos os $\vec{\psi}$ associados a uma solução de 4.4.23 tem $k-1$ variáveis livres. Isso acontece por que a matriz $\mathcal{J}_{\vec{F}}^{*}$ não tem informações suficientes para determinar $\vec{\psi}$ no caso geral e seria necessário olhar o jacobiano de $\vec{F}+\theta \vec{G}$ em um ponto fixo do modelo com ruído. Notamos que, com pequenas alterações, o mesmo acontece com o caso $\Omega=\varnothing$, de forma que podemos apenas determinar um espaço afim, com $k-1$ dimensões, aonde $\vec{\psi}$ deve estar.

Apesar disso, podemos concluir que para buscar pontos fixos robustos do modelo sem ruído, o importante é a condição $\bar{\Delta} \subseteq \Delta_{-}$e as soluções em que $\eta_{\Delta_{i}}^{*}>0$ para o sistema

\footnotetext{
${ }^{4} \mathrm{O}$ número de variáveis livres deve ser 1 (apenas o $\alpha_{i}$, no nosso caso), o que segue do teorema de RouchéCapelli (teorema A.2.3): Um sistema linear tem soluções se e somente se a matriz dos coeficientes $\left(\mathcal{J}_{\Delta_{i}}^{*}\right.$ no nosso caso) tem o mesmo posto que a matriz aumentada (isso é essencialmente o que satisfazer a equação 4.4.22 para um dado $i$ significa) e as soluções formam um espaço afim com dimensão igual ao número de variáveis $\left(\left|\Delta_{i}\right|\right)$ menos o posto da matriz dos coeficientes $\left(\left|\Delta_{i}\right|-1\right)$.
} 
4.4.23. Como $\overrightarrow{1}_{i} \cdot \mathcal{N}_{i} \cdot\left(\mathcal{J}_{\Omega}^{*}\right)^{-1}$ aparece na equação 4.4 .23 , iremos calcular explicitamente as suas coordenadas. Lembrando a equação 4.2.3, $\left(\mathcal{N}_{i}\right)_{\sigma, \sigma^{\prime}}=\eta_{\sigma}^{* 2} p_{\sigma^{\prime} \rightarrow \sigma}$, onde $\sigma \in \Delta_{i}$ e $\sigma^{\prime} \in \Omega$, de forma que

$$
\left(\overrightarrow{1}_{i} \cdot \mathcal{N}_{i} \cdot\left(\mathcal{J}_{\Omega}^{*}\right)^{-1}\right)_{\sigma}=\sum_{\sigma \in \Delta_{i}} \frac{\eta_{\sigma^{\prime}}^{* 2} p_{\sigma \rightarrow \sigma^{\prime}}}{\lambda_{\sigma}} .
$$

Para tornar as expressões mais compactas, iremos fazer a seguinte definição

$$
\xi_{i, \sigma}=\sum_{\sigma^{\prime} \in \Delta_{i}} \eta_{\sigma^{\prime}}^{* 2} p_{\sigma \rightarrow \sigma^{\prime}} \Rightarrow \lambda_{\sigma}=-\sum_{i} \xi_{i, \sigma} \forall \sigma \in \Omega,
$$

de forma que substituindo as equações 4.4 .25 e 4.4 .26 em 4.4.22, reescrevemos a equação de robustez como

$$
\sum_{\sigma \in \Delta_{i}} G_{\sigma}^{*}=\sum_{\sigma \in \Omega} \frac{\xi_{i, \sigma} G_{\sigma}^{*}}{\lambda_{\sigma}} \forall i
$$

Uma vez que já sabemos como tratar o caso geral, iremos passar para um caso menos abstrato, estudando o ruído não-uniforme da equação 4.4.10, ou seja, $G_{\sigma}=\sum_{\sigma^{\prime}}\left(\eta_{\sigma^{\prime}} n_{\sigma^{\prime} \rightarrow \sigma}-\right.$ $\left.\eta_{\sigma} n_{\sigma \rightarrow \sigma^{\prime}}\right)$. Por conveniência, iremos definir

$$
\phi_{i, \sigma}=\sum_{\sigma^{\prime} \in \Delta_{i}} \eta_{\sigma^{\prime}}^{*} n_{\sigma^{\prime} \rightarrow \sigma}
$$

Logo, a expressão para $G_{\sigma}^{*}$ se torna

$$
\begin{aligned}
& G_{\sigma}^{*}=\sum_{\sigma^{\prime}}\left(\eta_{\sigma^{\prime}}^{*} n_{\sigma^{\prime} \rightarrow \sigma}-\eta_{\sigma}^{*} n_{\sigma \rightarrow \sigma^{\prime}}\right)=\left(\sum_{i} \sum_{\sigma^{\prime} \in \Delta_{i}} \eta_{\sigma^{\prime}}^{*} n_{\sigma^{\prime} \rightarrow \sigma}\right)-\sum_{\sigma^{\prime}} \eta_{\sigma}^{*} n_{\sigma \rightarrow \sigma^{\prime}} \Rightarrow \\
& G_{\sigma}^{*}=\sum_{i} \phi_{i, \sigma}, \text { se } \sigma \in \Omega \quad \text { e } \quad \sum_{\sigma \in \Delta_{i}} G_{\sigma}^{*}=\sum_{\sigma \in \Delta_{i}} \sum_{j} \phi_{j, \sigma}-\sum_{\sigma} \phi_{i, \sigma} \forall i .
\end{aligned}
$$

Podemos usar esse resultado para escrever a equação de robustez com esse ruído, a partir de 4.4.27:

$$
\sum_{\sigma \in \Delta_{i}} \sum_{j} \phi_{j, \sigma}-\sum_{\sigma} \phi_{i, \sigma}-\sum_{\sigma \in \Omega} \frac{\xi_{i, \sigma}}{\lambda_{\sigma}} \sum_{j} \phi_{j, \sigma}=0 \forall i
$$

e as soluções $\eta_{\Delta_{i}}^{*}$ para essa equação, onde $\eta_{\Delta_{i}}^{*} \geq 0 \forall i$, dão os pontos fixos robustos. Para dar uma idéia da real complexidade dessa equação, iremos expandí-la em termos dos $\eta_{\Delta_{i}}^{*}$ (que são as variáveis) e das soluções de coexistência para as regras $\mathcal{R}_{\Delta_{i}}$. Se $\vec{\zeta}_{i}$ for a solução de coexistência para a regra $\mathcal{R}_{\Delta_{i}}$, então $\vec{\eta}_{i}^{*}=\eta_{\Delta_{i}}^{*} \cdot \vec{\zeta}_{i}$ e logo 


$$
\begin{gathered}
\phi_{i, \sigma}=\eta_{\Delta_{i}}^{*} \sum_{\sigma^{\prime} \in \Delta_{i}} \zeta_{\sigma^{\prime}} n_{\sigma^{\prime} \rightarrow \sigma}, \quad \xi_{i, \sigma}=\eta_{\Delta_{i}}^{* 2} \sum_{\sigma^{\prime} \in \Delta_{i}} \zeta_{\sigma^{\prime}} p_{\sigma \rightarrow \sigma^{\prime}} \mathrm{e} \\
\lambda_{\sigma}=-\sum_{i} \eta_{\Delta_{i}}^{* 2} \sum_{\sigma^{\prime} \in \Delta_{i}} \zeta_{\sigma^{\prime}} p_{\sigma \rightarrow \sigma^{\prime}} .
\end{gathered}
$$

Substituindo tudo na equação 4.4.30 temos

$$
\begin{gathered}
\eta_{\Delta_{i}}^{* 2} \sum_{\sigma^{\prime} \in \Omega} \frac{\sum_{j} \eta_{\Delta_{j}}^{*}\left(\sum_{\sigma \in \Delta_{i}} \zeta_{\sigma}^{2} p_{\sigma^{\prime} \rightarrow \sigma}\right)\left(\sum_{\sigma^{\prime \prime} \in \Delta_{j}} \zeta_{\sigma^{\prime \prime}} n_{\sigma^{\prime \prime} \rightarrow \sigma^{\prime}}\right)}{\sum_{j} \eta_{\Delta_{j}}^{* 2}\left(\sum_{\sigma^{\prime \prime} \in \Delta_{j}} \zeta_{\sigma^{\prime \prime}}^{2} p_{\sigma^{\prime} \rightarrow \sigma^{\prime \prime}}\right)}+ \\
-\eta_{\Delta_{i}}^{*}\left(\sum_{\sigma \in \Delta_{i}} \zeta_{\sigma} \sum_{\sigma^{\prime}} n_{\sigma \rightarrow \sigma^{\prime}}\right)+\sum_{j} \eta_{\Delta_{j}}^{*}\left(\sum_{\sigma^{\prime} \in \Delta_{j}} \zeta_{\sigma^{\prime}} \sum_{\sigma \in \Delta_{i}} n_{\sigma^{\prime} \rightarrow \sigma}\right)=0 .
\end{gathered}
$$

Note que o caso do ruído uniforme já está incluso como um caso particular e é obtido tomando $n_{\sigma \rightarrow \sigma^{\prime}}=1$, o que implica que $\phi_{i, \sigma}=\eta_{\Delta_{i}}^{*}$. Substituindo na equação de robustez para o ruído uniforme (ou seja, trocando $\phi_{i, \sigma}$ por $\eta_{\Delta_{i}}^{*}$ na equação 4.4.32) ela se simplifica para

$$
\begin{gathered}
\sum_{\sigma \in \Delta_{i}} \sum_{j} \eta_{\Delta_{j}}^{*}-\sum_{\sigma} \eta_{\Delta_{i}}^{*}-\sum_{\sigma \in \Omega} \frac{\xi_{i, \sigma}}{\lambda_{\sigma}} \sum_{j} \eta_{\Delta_{j}}^{*}=0 \Rightarrow \\
\left|\Delta_{i}\right|-M \eta_{\Delta_{i}}^{*}-\sum_{\sigma \in \Omega} \frac{\xi_{i, \sigma}}{\lambda_{\sigma}}=0 \forall i
\end{gathered}
$$

e expandindo $\xi_{i, \sigma}$, vem

$$
\eta_{\Delta_{i}}^{* 2} \sum_{\sigma^{\prime} \in \Omega} \frac{\sum_{\sigma \in \Delta_{i}} \zeta_{\sigma}^{2} p_{\sigma^{\prime} \rightarrow \sigma}}{\sum_{j} \eta_{\Delta_{j}}^{* 2}\left(\sum_{\sigma^{\prime \prime} \in \Delta_{j}} \zeta_{\sigma^{\prime \prime}}^{2} p_{\sigma^{\prime} \rightarrow \sigma^{\prime \prime}}\right)}-M \eta_{\Delta_{i}}^{*}+\left|\Delta_{i}\right|=0 .
$$

A primeira dificuldade para resolver as equações de robustez é que precisamos encontrar os $\vec{\zeta}_{i}$, o que envolve encontrar pontos fixos de coexistência do modelo sem ruído. Segundo, dados esses pontos fixos, precisamos resolver um sistema de equações não-lineares, definido pelas equações de robustez. Em particular, o denominador $\lambda_{\sigma}$ (que está presente desde as equações 4.4.27) torna problemático transformar as equações do sistema em polinômios, já que isso envolveria encontrar o mínimo múltiplo comum entre os $\lambda_{\sigma}$ com $\sigma \in \Omega$ e multiplicar as equações por ele. O problema surge porque, em geral, isso significa multiplicar as equações por $\prod_{\sigma \in \Omega} \lambda_{\sigma}$, que é um polinômio de grau $2|\Omega|$, o que dependendo do caso pode tornar a solução proibitiva.

A conclusão é que não podemos resolver essas equações analiticamente, a menos que estejamos em uma situação especial, em que os pesos da regra de confiança foram escolhidos cuidadosamente, para que os $\vec{\zeta}_{i}$ e os $\eta_{\Delta_{i}}^{*}$ pudessem ser encontrados; ou então, em alguns 
casos com poucas opiniões (3 ou 4). Iremos então nos focar no que deve acontecer com as variedades estáveis e instáveis dos pontos fixos robustos, após a adição de um ruído pequeno. Mais uma vez, faremos um tratamento com um ruído $\vec{G}$, abstrato, para depois examinar casos concretos. Se um ponto fixo é robusto, então ele deve sofrer uma variação pequena, quando o ruído é pequeno. As propriedades de estabilidade dele serão dadas pelo jacobiano $\mathcal{J}_{\vec{F}+\theta \vec{G}}=\mathcal{J}_{\vec{F}}+\theta \mathcal{J}_{\vec{G}}$. A nossa hipótese que $\vec{G}$ é suficientemente diferenciável implica que $\theta \mathcal{J}_{\vec{G}}$ será pequeno. Então, por continuidade, os autovalores de $\mathcal{J}_{\vec{F}}+\theta \mathcal{J}_{\vec{G}}$, avaliados no ponto fixo robusto (já deslocado, pela adição de ruído) são uma variação pequena dos autovalores de $\mathcal{J}_{\vec{F}}^{*}$. Em outras palavras, o espectro relevante é uma função contínua de $\theta$ (um raciocínio mais sofisticado mostra que ela também será diferenciável). Segue que os autovalores com parte real não-nula mantém o sinal da parte real, após a adição do ruído infinitesimal. Falta então apenas a análise do que acontece com os autovalores nulos previstos na seção 4.2.1.

Para descrever o que acontece com esses autovalores, iremos usar o formalismo desenvolvido no apêndice A.3, sobre teoria de perturbações em matrizes gerais. O que faremos é escrever $\mathcal{J}_{\vec{F}}+\theta \mathcal{J}_{\vec{G}}$, avaliado no ponto fixo, expandindo até a primeira ordem em $\theta$, para chegarmos em uma matriz $\mathcal{J}_{\vec{F}}^{*}+\theta P$. Como veremos, $P$ terá 2 contribuições, a variação de $\mathcal{J}_{\vec{F}}$ por causa do deslocamento do ponto fixo e o jacobiano $\mathcal{J}_{\vec{G}}$ avaliado no ponto fixo sem o deslocamento. O formalismo que usaremos é até certo ponto semelhante ao que é feito nas teorias de perturbação de primeira ordem, no caso degenerado, em mecânica quântica não-relativística, porém com adaptações para matrizes que não sejam hermitianas. Precisamos para tanto utilizar os autovetores à esquerda e à direita de $\mathcal{J}_{\vec{F}}^{*}$, que tenham autovalor 0 . Usando as equações $4.2 .5,4.2 .14$ e 4.2.8, vemos que os autovetores à direita, $\vec{v}^{(i)}$, são combinações lineares de

$$
\vec{v}^{(1)} \equiv\left[\begin{array}{c}
\vec{\zeta}_{1} \\
\overrightarrow{0}_{2} \\
\overrightarrow{0}_{3} \\
\vdots \\
\overrightarrow{0}_{k} \\
\overrightarrow{0}_{\Omega}
\end{array}\right], \vec{v}^{(2)} \equiv\left[\begin{array}{c}
\overrightarrow{0}_{1} \\
\vec{\zeta}_{2} \\
\overrightarrow{0}_{3} \\
\vdots \\
\overrightarrow{0}_{k} \\
\overrightarrow{0}_{\Omega}
\end{array}\right], \vec{v}^{(3)} \equiv\left[\begin{array}{c}
\overrightarrow{0}_{1} \\
\overrightarrow{0}_{2} \\
\vec{\zeta}_{3} \\
\vdots \\
\overrightarrow{0}_{k} \\
\overrightarrow{0}_{\Omega}
\end{array}\right], \ldots, \vec{v}^{(k)} \equiv\left[\begin{array}{c}
\overrightarrow{0}_{1} \\
\overrightarrow{0}_{2} \\
\overrightarrow{0}_{3} \\
\vdots \\
\overrightarrow{\vec{\zeta}_{k}} \\
\overrightarrow{0}_{\Omega}
\end{array}\right] .
$$

Segue das equações 4.2.7 e 4.2.15 que os autovetores à esquerda, $\vec{u}^{(i)}$, podem ser escolhidos de forma que $\vec{u}^{(i)} \cdot \vec{v}^{(j)}=\delta_{i, j}$, bastando definí-los como

$$
\begin{gathered}
\vec{u}^{(1)} \equiv\left[\begin{array}{llllll}
\overrightarrow{1}_{1} & \overrightarrow{0}_{2} & \overrightarrow{0}_{3} & \ldots & \overrightarrow{0}_{k} & \vec{y}^{(1)}
\end{array}\right], \vec{u}^{(2)} \equiv\left[\begin{array}{lllllll}
\overrightarrow{0}_{1} & \overrightarrow{1}_{2} & \overrightarrow{0}_{3} & \ldots & \overrightarrow{0}_{k} & \vec{y}^{(2)}
\end{array}\right], \\
\vec{u}^{(3)} \equiv\left[\begin{array}{llllllll}
\overrightarrow{0}_{1} & \overrightarrow{0}_{2} & \overrightarrow{1}_{3} & \ldots & \overrightarrow{0}_{k} & \vec{y}^{(3)}
\end{array}\right], \ldots, \vec{u}^{(k)} \equiv\left[\begin{array}{lllllll}
\overrightarrow{0}_{1} & \overrightarrow{0}_{2} & \overrightarrow{0}_{3} & \ldots & \overrightarrow{1}_{k} & \vec{y}^{(k)}
\end{array}\right],
\end{gathered}
$$

onde as coordenadas dos vetores $\vec{y}^{(i)}$ correspondem às opiniões do conjunto $\Omega$. Para determinar essas coordenadas que estão faltando usamos a equação 4.4.16, que implica que se $\vec{u}^{(i)} \cdot \mathcal{J}_{\vec{F}}^{*}=\overrightarrow{0}$, então 


$$
\overrightarrow{1}_{i} \cdot \mathcal{N}_{i}+\vec{y}^{(i)} \cdot \mathcal{J}_{\Omega}^{*}=\overrightarrow{0}_{\Omega} .
$$

Como se trata de um ponto fixo robusto, então $\mathcal{J}_{\Omega}^{*}$ é inversível e podemos resolver a equação:

$$
\vec{y}^{(i)}=-\overrightarrow{1}_{i} \cdot \mathcal{N}_{i} \cdot\left(\mathcal{J}_{\Omega}^{*}\right)^{-1} \Rightarrow\left(\vec{y}^{(i)}\right)_{\sigma}=\frac{-\xi_{i, \sigma}}{\lambda_{\sigma}} .
$$

Chamamos a atenção que, se $\Omega=\varnothing$, então não precisamos nos preocupar com isso, já que os $\vec{y}^{(i)}$ não aparecem.

Após a adição de um ruído $\theta$, o ponto fixo robusto é deslocado de $\vec{\eta}^{*}$ para $\vec{\eta}^{*}+\theta \vec{\psi}$. O último ingrediente que precisamos para aplicar nosso tratamento perturbativo é o jacobiano $\mathcal{J}_{\vec{F}}+\theta \mathcal{J}_{\vec{G}}$, avaliado em $\vec{\eta}^{*}+\theta \vec{\psi}$, mantendo apenas termos até a primeira ordem em $\theta$. Ou seja, precisamos expandir

$$
\begin{gathered}
\mathcal{J}_{\vec{F}}\left(\vec{\eta}^{*}+\theta \vec{\psi}\right)+\theta \mathcal{J}_{\vec{G}}\left(\vec{\eta}^{*}+\theta \vec{\psi}\right)= \\
=\mathcal{J}_{\vec{F}}\left(\vec{\eta}^{*}\right)+\theta \sum_{\sigma} \psi_{\sigma}\left(\frac{\partial}{\partial \eta_{\sigma}} \mathcal{J}_{\vec{F}}\right)\left(\vec{\eta}^{*}\right)+\theta \mathcal{J}_{\vec{G}}\left(\vec{\eta}^{*}\right)+\mathcal{O}\left(\theta^{2}\right) .
\end{gathered}
$$

Como deduzimos no apêndice A.3, as derivadas com respeito a $\theta$ dos autovalores nulos serão os autovalores da matriz $\mathfrak{G}^{5}$, cujos termos são

$$
\mathfrak{G}_{i, j}=\vec{u}^{(i)}\left(\sum_{\sigma} \psi_{\sigma}\left(\frac{\partial}{\partial \eta_{\sigma}} \mathcal{J}_{\vec{F}}\right)\left(\vec{\eta}^{*}\right)+\mathcal{J}_{\vec{G}}\left(\vec{\eta}^{*}\right)\right) \vec{v}^{(j)} .
$$

Para os próximos cálculos faremos as seguintes definições

$$
\widetilde{\zeta}^{(1)} \equiv\left[\begin{array}{c}
\vec{\zeta}_{1} \\
\overrightarrow{0}_{2} \\
\overrightarrow{0}_{3} \\
\vdots \\
\overrightarrow{0}_{k}
\end{array}\right], \widetilde{\zeta}^{(2)} \equiv\left[\begin{array}{c}
\overrightarrow{0}_{1} \\
\vec{\zeta}_{2} \\
\overrightarrow{0}_{3} \\
\vdots \\
\overrightarrow{0}_{k}
\end{array}\right], \widetilde{\zeta}^{(3)} \equiv\left[\begin{array}{c}
\overrightarrow{0}_{1} \\
\overrightarrow{0}_{2} \\
\overrightarrow{\zeta_{3}} \\
\vdots \\
\overrightarrow{0}_{k}
\end{array}\right], \ldots, \widetilde{\zeta}^{(k)} \equiv\left[\begin{array}{c}
\overrightarrow{0}_{1} \\
\overrightarrow{0}_{2} \\
\overrightarrow{0}_{3} \\
\vdots \\
\vec{\zeta}_{k}
\end{array}\right]
$$

\footnotetext{
${ }^{5}$ Mais precisamente, o que fizemos nesse apêndice foi considerar uma matriz $M$ e uma perturbação $\alpha N$, onde $\alpha$ é pequeno. Nós mostramos que se $\lambda$ é um autovalor, com multiplicidade algébrica $\mu$ e $\mu$ autovetores à esquerda e à direita, $\vec{u}_{i}$ e $\vec{v}_{i}$, respectivamente, tais que $\vec{u}_{i} \cdot \vec{v}_{j}=\delta_{i, j}$ (o que é equivalente, pelo teorema A.2.8, a dizer que a multiplicidade geométrica também é $\mu$ ), então as derivadas desses autovalores com respeito a $\alpha$ (quando $\alpha=0$ ) são dadas pelos autovalores da matriz $\Lambda$, cujos termos são $\Lambda_{i, j}=\vec{u}_{i} \cdot N . \vec{v}_{j}$. Esse é essencialmente o mesmo resultado que o caso particular de uma teoria de perturbação degenerada em que $M$ e $N$ sejam hermitianas e $\alpha$ seja sempre real (que é comum em mecânica quântica não-relativística. Note que no jargão da mecânica quântica, os autovetores à esquerda são os bras e os à direita são os kets). Porém, na dedução do caso degenerado geral, temos que lidar com o fato que sempre existe uma matriz $N$ que torna $M+\alpha N$ não diagonalizável, quando $\alpha \neq 0$.
} 
ou seja, quando nos referirmos a $\widetilde{\zeta}^{(i)}$ estaremos nos referindo a um vetor, cujas componentes correspondem a opiniões em $\Delta$, onde as componentes correspondentes a $\Delta_{i}$ dão $\vec{\zeta}_{i}$ e as outras são 0 . De forma análoga, definiremos

$$
\widetilde{1}^{(1)} \equiv\left[\begin{array}{llll}
\overrightarrow{1}_{1} & \overrightarrow{0}_{2} & \ldots & \overrightarrow{0}_{k}
\end{array}\right], \widetilde{1}^{(2)} \equiv\left[\begin{array}{llll}
\overrightarrow{0}_{1} & \overrightarrow{1}_{2} & \ldots & \overrightarrow{0}_{k}
\end{array}\right], \ldots, \widetilde{1}^{(k)} \equiv\left[\begin{array}{llll}
\overrightarrow{0}_{1} & \overrightarrow{0}_{2} & \ldots & \overrightarrow{1}_{k}
\end{array}\right]
$$

Iremos calcular $\vec{u}_{i} \cdot M \cdot \vec{v}_{j}$ e iremos expressar $M$ em blocos,

$$
M=\left[\begin{array}{ll}
M_{\Delta, \Delta} & M_{\Delta, \Omega} \\
M_{\Omega, \Delta} & M_{\Omega, \Omega}
\end{array}\right],
$$

onde $M_{X, Y}$ denota o bloco de $M$, em que as colunas estão em $X$ e as linhas estão em $Y$. Logo:

$$
\begin{gathered}
\vec{u}_{i} \cdot M \cdot \vec{v}_{j}=\left[\begin{array}{cc}
\widetilde{1}^{(i)} & \vec{y}^{(i)}
\end{array}\right] \cdot\left[\begin{array}{ll}
M_{\Delta, \Delta} & M_{\Delta, \Omega} \\
M_{\Omega, \Delta} & M_{\Omega, \Omega}
\end{array}\right] \cdot\left[\begin{array}{c}
\widetilde{\zeta}^{(j)} \\
\overrightarrow{0}_{\Omega}
\end{array}\right]=\left[\begin{array}{ll}
\widetilde{1}^{(i)} & \vec{y}^{(i)}
\end{array}\right] \cdot\left[\begin{array}{c}
M_{\Delta, \Delta} \cdot \widetilde{\zeta}^{(j)} \\
M_{\Omega, \Delta} \cdot \widetilde{\zeta}^{(j)}
\end{array}\right] \Rightarrow \\
\vec{u}_{i} \cdot M \cdot \vec{v}_{j}=\widetilde{1}^{(i)} \cdot M_{\Delta, \Delta} \cdot \widetilde{\zeta}^{(j)}+\vec{y}^{(i)} \cdot M_{\Omega, \Delta} \cdot \widetilde{\zeta}^{(j)} .
\end{gathered}
$$

A primeira coisa que notamos é que os termos dos blocos $M_{\Delta, \Omega}$ e $M_{\Omega, \Omega}$ não são relevantes. Para calcular os termos de $\mathfrak{G}$, iremos dividir a matriz $M$ relevante em 2 partes, de forma que $M=M^{(1)}+M^{(2)}$, onde

$$
M^{(1)}=\sum_{\sigma} \psi_{\sigma}\left(\frac{\partial}{\partial \eta_{\sigma}} \mathcal{J}_{\vec{F}}\right)\left(\vec{\eta}^{*}\right)
$$

é a parte devida a modificação de $\mathcal{J}_{\vec{F}}$, causada pelo deslocamento $\theta \vec{\psi}$ do ponto fixo robusto $\mathrm{e}$

$$
M^{(2)}=\mathcal{J}_{\vec{G}}\left(\vec{\eta}^{*}\right)
$$

dá o efeito do jacobiano de $\vec{G}$, que é avaliado no ponto fixo sem deslocamento, pois esse deslocamento só causa um termo de ordem superior nesse caso. Essas 2 matrizes resultarão nas matrizes $\mathfrak{G}^{(1)}$ e $\mathfrak{G}^{(2)}$, onde $\mathfrak{G}=\mathfrak{G}^{(1)}+\mathfrak{G}^{(2)}$. Depois, notamos que $M^{(1)}$ depende de $\vec{\psi}$. Como não conseguimos determinar completamente os $\vec{\psi}_{i}$, iremos primeiro mostrar que somente as coordenadas de $\vec{\psi}_{\Omega}$ são necessárias. Os termos de $M^{(1)}$ são

$$
M_{\sigma, \sigma^{\prime}}^{(1)}=\sum_{\sigma^{\prime \prime}} \psi_{\sigma^{\prime \prime}}\left(\frac{\partial \mathcal{J}_{\vec{F}}}{\partial \eta_{\sigma^{\prime \prime}}}\right)_{\sigma, \sigma^{\prime}}\left(\vec{\eta}^{*}\right)=\sum_{\sigma^{\prime \prime}} \psi_{\sigma^{\prime \prime}}\left(\frac{\partial^{2} F_{\sigma}}{\partial \eta_{\sigma^{\prime}} \partial \eta_{\sigma^{\prime \prime}}}\right)\left(\vec{\eta}^{*}\right) .
$$

Lembramos a expressão de $\partial^{2} F_{\sigma} / \partial \eta_{\sigma^{\prime}} \partial \eta_{\sigma^{\prime \prime}}$, dada pela equação 4.2.21: 


$$
\begin{aligned}
\frac{\partial^{2} F_{\sigma}}{\partial \eta_{\sigma^{\prime}} \partial \eta_{\sigma^{\prime \prime}}} & =2 \delta_{\sigma, \sigma^{\prime}, \sigma^{\prime \prime}} \sum_{\sigma^{\prime \prime \prime}} \eta_{\sigma^{\prime \prime \prime}} p_{\sigma^{\prime \prime \prime} \rightarrow \sigma}+2 \delta_{\sigma, \sigma^{\prime}}\left(\eta_{\sigma} p_{\sigma^{\prime \prime} \rightarrow \sigma}-\eta_{\sigma^{\prime \prime}} p_{\sigma \rightarrow \sigma^{\prime \prime}}\right)+ \\
& +2 \delta_{\sigma, \sigma^{\prime \prime}}\left(\eta_{\sigma} p_{\sigma^{\prime} \rightarrow \sigma}-\eta_{\sigma^{\prime}} p_{\sigma \rightarrow \sigma^{\prime}}\right)-2 \delta_{\sigma^{\prime}, \sigma^{\prime \prime}} \eta_{\sigma} p_{\sigma \rightarrow \sigma^{\prime}} .
\end{aligned}
$$

Para o bloco $M_{\Omega, \Delta}^{(1)}$, consideramos essa expressão para $\sigma \in \Omega$

$$
\frac{\partial^{2} F_{\sigma}}{\partial \eta_{\sigma^{\prime}} \partial \eta_{\sigma^{\prime \prime}}}\left(\vec{\eta}^{*}\right)=2 \delta_{\sigma, \sigma^{\prime}, \sigma^{\prime \prime}} \sum_{\sigma^{\prime \prime \prime} \in \Delta} \eta_{\sigma^{\prime \prime \prime}}^{*} p_{\sigma^{\prime \prime \prime} \rightarrow \sigma}-2 \delta_{\sigma, \sigma^{\prime}} \eta_{\sigma^{\prime \prime}}^{*} p_{\sigma \rightarrow \sigma^{\prime \prime}}-2 \delta_{\sigma, \sigma^{\prime \prime}} \eta_{\sigma^{\prime}}^{*} p_{\sigma \rightarrow \sigma^{\prime}}
$$

Portanto, os coeficientes de $M_{\Omega, \Delta}^{(1)}$ serão

$$
\begin{gathered}
\sum_{\sigma^{\prime \prime}} \psi_{\sigma^{\prime \prime}}\left(\frac{\partial^{2} F_{\sigma}}{\partial \eta_{\sigma^{\prime}} \partial \eta_{\sigma^{\prime \prime}}}\right)\left(\vec{\eta}^{*}\right)=2 \delta_{\sigma, \sigma^{\prime}} \psi_{\sigma} \sum_{\sigma^{\prime \prime} \in \Delta} \eta_{\sigma^{\prime \prime}}^{*} p_{\sigma^{\prime \prime} \rightarrow \sigma}+ \\
-2 \delta_{\sigma, \sigma^{\prime}} \sum_{\sigma^{\prime \prime} \in \Delta} \psi_{\sigma^{\prime \prime}} \eta_{\sigma^{\prime \prime}}^{*} p_{\sigma \rightarrow \sigma^{\prime \prime}}-2 \psi_{\sigma} \eta_{\sigma^{\prime}}^{*} p_{\sigma \rightarrow \sigma^{\prime}}
\end{gathered}
$$

Como $\sigma \in \Omega$, mas $\sigma^{\prime} \in \Delta$, segue que $\delta_{\sigma, \sigma^{\prime}}=0$ e a expressão se simplifica para

$$
M_{\sigma, \sigma^{\prime}}^{(1)}=-2 \psi_{\sigma} \eta_{\sigma^{\prime}}^{*} p_{\sigma \rightarrow \sigma^{\prime}}
$$

Para o bloco $M_{\Delta, \Delta}^{(1)}$, devemos considerar a expressão quando $\sigma \in \Delta$

$$
\begin{gathered}
\sum_{\sigma^{\prime \prime}} \psi_{\sigma^{\prime \prime}}\left(\frac{\partial^{2} F_{\sigma}}{\partial \eta_{\sigma^{\prime}} \partial \eta_{\sigma^{\prime \prime}}}\right)\left(\vec{\eta}^{*}\right)=2 \delta_{\sigma, \sigma^{\prime}}\left(\psi_{\sigma} \sum_{\sigma^{\prime \prime} \in \Delta} \eta_{\sigma^{\prime \prime}}^{*} p_{\sigma^{\prime \prime} \rightarrow \sigma}+\right. \\
\left.+\sum_{\sigma^{\prime \prime}} \psi_{\sigma^{\prime \prime}}\left(\eta_{\sigma}^{*} p_{\sigma^{\prime \prime} \rightarrow \sigma}-\eta_{\sigma^{\prime \prime}}^{*} p_{\sigma \rightarrow \sigma^{\prime \prime}}\right)\right)+2 \psi_{\sigma}\left(\eta_{\sigma}^{*} p_{\sigma^{\prime} \rightarrow \sigma}-\eta_{\sigma^{\prime}}^{*} p_{\sigma \rightarrow \sigma^{\prime}}\right)-2 \psi_{\sigma^{\prime}} \eta_{\sigma}^{*} p_{\sigma \rightarrow \sigma^{\prime}} .
\end{gathered}
$$

Como $\sigma, \sigma^{\prime} \in \Delta$, iremos nos focar no que acontece quando $\sigma \in \Delta_{i}$ e $\sigma^{\prime} \in \Delta_{j}$. Nesse caso, temos que $\delta_{\sigma, \sigma^{\prime}}, p_{\sigma \rightarrow \sigma^{\prime}}$ e $p_{\sigma^{\prime} \rightarrow \sigma}$ são 0 se $\delta_{i, j}=0$. Para aproveitar essa propriedade, a soma sobre $\sigma^{\prime \prime} \in \Delta$ pode ser reescrita como uma soma em $k$ da soma sobre $\sigma^{\prime \prime} \in \Delta_{k}$ e a outra soma, sobre $\sigma^{\prime \prime}$, pode ser reescrita como uma soma sobre $\sigma^{\prime \prime} \in \Omega$ e $\sigma^{\prime \prime} \in \Delta$ (que também pode ser quebrada em uma soma sobre $k$ e $\Delta_{k}$ )

$$
\begin{aligned}
& \sum_{\sigma^{\prime \prime}} \psi_{\sigma^{\prime \prime}}\left(\frac{\partial^{2} F_{\sigma}}{\partial \eta_{\sigma^{\prime}} \partial \eta_{\sigma^{\prime \prime}}}\right)\left(\vec{\eta}^{*}\right)=2 \delta_{\sigma, \sigma^{\prime}}\left(\psi_{\sigma} \sum_{k} \sum_{\sigma^{\prime \prime} \in \Delta_{k}} \eta_{\sigma^{\prime \prime}}^{*} p_{\sigma^{\prime \prime} \rightarrow \sigma}+\sum_{\sigma^{\prime \prime} \in \Omega} \psi_{\sigma^{\prime \prime}} \eta_{\sigma}^{*} p_{\sigma^{\prime \prime} \rightarrow \sigma}+\right. \\
& \left.+\sum_{k} \sum_{\sigma^{\prime \prime} \in \Delta_{k}} \psi_{\sigma^{\prime \prime}}\left(\eta_{\sigma}^{*} p_{\sigma^{\prime \prime} \rightarrow \sigma}-\eta_{\sigma^{\prime \prime}}^{*} p_{\sigma \rightarrow \sigma^{\prime \prime}}\right)\right)+2 \psi_{\sigma}\left(\eta_{\sigma}^{*} p_{\sigma^{\prime} \rightarrow \sigma}-\eta_{\sigma^{\prime}}^{*} p_{\sigma \rightarrow \sigma^{\prime}}\right)-2 \psi_{\sigma^{\prime}} \eta_{\sigma}^{*} p_{\sigma \rightarrow \sigma^{\prime}}
\end{aligned}
$$


Adicionando explicitamente os deltas de Kronecker que discutimos anteriormente

$$
\begin{aligned}
& 2 \delta_{\sigma, \sigma^{\prime}} \delta_{i, j}\left(\psi_{\sigma} \sum_{k} \delta_{i, k} \sum_{\sigma^{\prime \prime} \in \Delta_{k}} \eta_{\sigma^{\prime \prime}}^{*} p_{\sigma^{\prime \prime} \rightarrow \sigma}+\sum_{k} \delta_{i, k} \sum_{\sigma^{\prime \prime} \in \Delta_{k}} \psi_{\sigma^{\prime \prime}}\left(\eta_{\sigma}^{*} p_{\sigma^{\prime \prime} \rightarrow \sigma}-\eta_{\sigma^{\prime \prime}}^{*} p_{\sigma \rightarrow \sigma^{\prime \prime}}\right)+\right. \\
& \left.\quad+\sum_{\sigma^{\prime \prime} \in \Omega} \psi_{\sigma^{\prime \prime}} \eta_{\sigma}^{*} p_{\sigma^{\prime \prime} \rightarrow \sigma}\right)+2 \delta_{i, j} \psi_{\sigma}\left(\eta_{\sigma}^{*} p_{\sigma^{\prime} \rightarrow \sigma}-\eta_{\sigma^{\prime}}^{*} p_{\sigma \rightarrow \sigma^{\prime}}\right)-2 \delta_{i, j} \psi_{\sigma^{\prime}} \eta_{\sigma}^{*} p_{\sigma \rightarrow \sigma^{\prime}},
\end{aligned}
$$

de onde segue, simplificando as somas com os deltas

$$
\begin{gathered}
\sum_{\sigma^{\prime \prime}} \psi_{\sigma^{\prime \prime}}\left(\frac{\partial^{2} F_{\sigma}}{\partial \eta_{\sigma^{\prime}} \partial \eta_{\sigma^{\prime \prime}}}\right)\left(\vec{\eta}^{*}\right)=2 \delta_{i, j}\left(\psi_{\sigma} \delta_{\sigma, \sigma^{\prime}} \sum_{\sigma^{\prime \prime} \in \Delta_{i}} \eta_{\sigma^{\prime \prime}}^{*} p_{\sigma^{\prime \prime} \rightarrow \sigma}+\right. \\
+\delta_{\sigma, \sigma^{\prime}} \sum_{\sigma^{\prime \prime} \in \Delta_{i}} \psi_{\sigma^{\prime \prime}}\left(\eta_{\sigma}^{*} p_{\sigma^{\prime \prime} \rightarrow \sigma}-\eta_{\sigma^{\prime \prime}}^{*} p_{\sigma \rightarrow \sigma^{\prime \prime}}\right)+\delta_{\sigma, \sigma^{\prime}} \sum_{\sigma^{\prime \prime} \in \Omega} \psi_{\sigma^{\prime \prime}} \eta_{\sigma}^{*} p_{\sigma^{\prime \prime} \rightarrow \sigma}+ \\
\left.+\psi_{\sigma}\left(\eta_{\sigma}^{*} p_{\sigma^{\prime} \rightarrow \sigma}-\eta_{\sigma^{\prime}}^{*} p_{\sigma \rightarrow \sigma^{\prime}}\right)-\psi_{\sigma^{\prime}} \eta_{\sigma}^{*} p_{\sigma \rightarrow \sigma^{\prime}}\right) .
\end{gathered}
$$

Podemos ver que os termos de $M_{\Omega, \Delta}^{(1)}$ dependem apenas de $\vec{\psi}_{\Omega}$ (equação 4.4.38), porém os termos de $M_{\Delta, \Delta}^{(1)}$ dependem explicitamente de todas as coordenadas de $\vec{\psi}$ (equação 4.4.39). Vamos então calcular $\widetilde{1}^{(i)} \cdot M_{\Delta, \Delta}^{(1)} \cdot \widetilde{\zeta}^{(j)}$, que é o que vai contribuir de fato para $\mathfrak{G}^{(1)}$ :

$$
\widetilde{1}^{(i)} \cdot M_{\Delta, \Delta}^{(1)} \cdot \widetilde{\zeta}^{(j)}=\sum_{\sigma \in \Delta_{i}} \sum_{\sigma^{\prime} \in \Delta_{j}} M_{\sigma, \sigma^{\prime}}^{(1)} \zeta_{\sigma^{\prime}}
$$

Da equação 4.4.39, temos que esse termo só é não-nulo se $i=j$, logo

$$
\begin{aligned}
& \widetilde{1}^{(i)} \cdot M_{\Delta, \Delta}^{(1)} \cdot \widetilde{\zeta}^{(j)}=2 \delta_{i, j} \sum_{\sigma^{\prime} \in \Delta_{i}} \zeta_{\sigma^{\prime}}\left(\psi_{\sigma^{\prime}} \sum_{\sigma^{\prime \prime} \in \Delta_{i}} \eta_{\sigma^{\prime \prime}}^{*} p_{\sigma^{\prime \prime} \rightarrow \sigma^{\prime}}+\right. \\
& +\sum_{\sigma^{\prime \prime} \in \Delta_{i}} \psi_{\sigma^{\prime \prime}}\left(\eta_{\sigma^{\prime}}^{*} p_{\sigma^{\prime \prime} \rightarrow \sigma^{\prime}}-\eta_{\sigma^{\prime \prime}}^{*} p_{\sigma^{\prime} \rightarrow \sigma^{\prime \prime}}\right)+\sum_{\sigma^{\prime \prime} \in \Omega} \psi_{\sigma^{\prime \prime}} \eta_{\sigma^{\prime}}^{*} p_{\sigma^{\prime \prime} \rightarrow \sigma^{\prime}}+ \\
& \left.\quad+\sum_{\sigma \in \Delta_{i}} \psi_{\sigma}\left(\eta_{\sigma}^{*} p_{\sigma^{\prime} \rightarrow \sigma}-\eta_{\sigma^{\prime}}^{*} p_{\sigma \rightarrow \sigma^{\prime}}\right)-\psi_{\sigma^{\prime}} \sum_{\sigma \in \Delta_{i}} \eta_{\sigma}^{*} p_{\sigma \rightarrow \sigma^{\prime}}\right) .
\end{aligned}
$$

Temos agora que todas as somas sobre $\Delta_{i}$ se cancelam. Para tornar isso mais claro, iremos trocar as somas sobre $\sigma^{\prime \prime}$ por somas em $\sigma$ e agrupar as somas sobre $\Delta_{i}$ 


$$
\begin{aligned}
\widetilde{1}^{(i)} \cdot M_{\Delta, \Delta}^{(1)} \cdot \widetilde{\zeta}^{(j)} & =2 \delta_{i, j} \sum_{\sigma^{\prime} \in \Delta_{i}} \zeta_{\sigma^{\prime}}\left(\sum _ { \sigma \in \Delta _ { i } } \left(\psi_{\sigma^{\prime}} \eta_{\sigma}^{*} p_{\sigma \rightarrow \sigma^{\prime}}+\psi_{\sigma}\left(\eta_{\sigma^{\prime}}^{*} p_{\sigma \rightarrow \sigma^{\prime}}-\eta_{\sigma}^{*} p_{\sigma^{\prime} \rightarrow \sigma}\right)+\right.\right. \\
& \left.\left.+\psi_{\sigma}\left(\eta_{\sigma}^{*} p_{\sigma^{\prime} \rightarrow \sigma}-\eta_{\sigma^{\prime}}^{*} p_{\sigma \rightarrow \sigma^{\prime}}\right)-\psi_{\sigma^{\prime}} \eta_{\sigma}^{*} p_{\sigma \rightarrow \sigma^{\prime}}\right)+\sum_{\sigma \in \Omega} \psi_{\sigma} \eta_{\sigma^{\prime}}^{*} p_{\sigma \rightarrow \sigma^{\prime}}\right)
\end{aligned}
$$

Após esses cancelamentos, $\widetilde{1}^{(i)} \cdot M_{\Delta, \Delta}^{(1)} \cdot \widetilde{\zeta}^{(j)}$ também só depende dos $\vec{\psi}_{\Omega}$ e logo, já possuimos todas as informações necessárias para seguir em frente.

$$
\widetilde{1}^{(i)} . M_{\Delta, \Delta}^{(1)} \cdot \widetilde{\zeta}^{(j)}=2 \delta_{i, j} \sum_{\sigma \in \Omega} \sum_{\sigma^{\prime} \in \Delta_{i}} \zeta_{\sigma^{\prime}} \psi_{\sigma} \eta_{\sigma^{\prime}}^{*} p_{\sigma \rightarrow \sigma^{\prime}}=\frac{-2 \delta_{i, j}}{\eta_{\Delta_{i}}^{*}} \sum_{\sigma \in \Omega} \frac{\xi_{i, \sigma} G_{\sigma}^{*}}{\lambda_{\sigma}}
$$

Para terminar de calcular $\mathfrak{G}^{(1)}$, vamos calcular $\vec{y}^{(i)} \cdot M_{\Omega, \Delta}^{(1)} \cdot \widetilde{\zeta}^{(j)}$. Substituindo a solução para $\vec{\psi}_{\Omega}$ (ou seja $\psi_{\sigma}=-G_{\sigma}^{*} / \lambda_{\sigma}$ ) em 4.4.38 temos

$$
M_{\sigma, \sigma^{\prime}}^{(1)}=\frac{2 G_{\sigma}^{*} \eta_{\sigma^{\prime}}^{*} p_{\sigma \rightarrow \sigma^{\prime}}}{\lambda_{\sigma}}
$$

e $\log 0$

$$
\vec{y}^{(i)} \cdot M_{\Omega, \Delta}^{(1)} \cdot \widetilde{\zeta}^{(j)}=\sum_{\sigma \in \Omega} \frac{-\xi_{i, \sigma}}{\lambda_{\sigma}} \sum_{\sigma^{\prime} \in \Delta_{j}} \frac{2 G_{\sigma}^{*} \eta_{\sigma^{\prime}}^{*} p_{\sigma \rightarrow \sigma^{\prime}} \zeta_{\sigma^{\prime}}}{\lambda_{\sigma}}=\frac{-2}{\eta_{\Delta_{j}}^{*}} \sum_{\sigma \in \Omega} \frac{\xi_{i, \sigma} \xi_{j, \sigma} G_{\sigma}^{*}}{\lambda_{\sigma}^{2}}
$$

Juntando os dois termos, 4.4.40 e 4.4.41, chegamos em

$$
\mathfrak{G}_{i, j}^{(1)}=-2 \sum_{\sigma \in \Omega} \frac{\xi_{i, \sigma} G_{\sigma}^{*}}{\lambda_{\sigma} \eta_{\Delta_{j}}^{*}}\left(\delta_{i, j}+\frac{\xi_{j, \sigma}}{\lambda_{\sigma}}\right)
$$

Finalmente, note que no caso $\Omega=\varnothing$, teriamos apenas o termo proveniente do bloco $M_{\Delta, \Delta}^{(1)}$ (que corresponderia na verdade a toda a matriz $M^{(1)}$ ) e ele não teria uma soma sobre $\Omega$, de forma que os cancelamentos que nos levaram a 4.4.40 significam que, nesse caso, $\mathfrak{G}^{(1)}=0$.

Notamos que essa matriz $\mathfrak{G}^{(1)}$ possui duas propriedades interessantes

$$
\sum_{i} \mathfrak{G}_{i, j}^{(1)}=-2 \sum_{\sigma \in \Omega} \sum_{i} \frac{\xi_{i, \sigma} G_{\sigma}^{*}}{\lambda_{\sigma} \eta_{\Delta_{j}}^{*}}\left(\delta_{i, j}+\frac{\xi_{j, \sigma}}{\lambda_{\sigma}}\right)=-2 \sum_{\sigma \in \Omega} \frac{\xi_{j, \sigma} G_{\sigma}^{*}}{\lambda_{\sigma} \eta_{\Delta_{j}}^{*}}\left(1+\sum_{i} \frac{\xi_{i, \sigma}}{\lambda_{\sigma}}\right) .
$$

Mas segue da equação 4.4.26 que 


$$
1+\sum_{i} \frac{\xi_{i, \sigma}}{\lambda_{\sigma}}=0
$$

e logo

$$
\sum_{i} \mathfrak{G}_{i, j}^{(1)}=0
$$

Da mesma forma,

$$
\sum_{j} \mathfrak{G}_{i, j}^{(1)} \eta_{\Delta_{j}}^{*}=-2 \sum_{\sigma \in \Omega} \sum_{j} \frac{\xi_{i, \sigma} G_{\sigma}^{*}}{\lambda_{\sigma}}\left(\delta_{i, j}+\frac{\xi_{j, \sigma}}{\lambda_{\sigma}}\right)=-2 \sum_{\sigma \in \Omega} \frac{\xi_{i, \sigma} G_{\sigma}^{*}}{\lambda_{\sigma}}\left(1+\sum_{j} \frac{\xi_{j, \sigma}}{\lambda_{\sigma}}\right)
$$

e novamente pela equação 4.4 .43

$$
\sum_{j} \mathfrak{G}_{i, j}^{(1)} \eta_{\Delta_{j}}^{*}=0
$$

Definindo então um vetor $\vec{H}=\left(\eta_{\Delta_{1}}^{*}, \ldots, \eta_{\Delta_{k}}^{*}\right)$, essas propriedades podem ser expressas como

$$
\overrightarrow{1} \cdot \mathfrak{G}^{(1)}=\overrightarrow{0} \quad \text { e } \quad \mathfrak{G}^{(1)} \cdot \vec{H}=\overrightarrow{0} .
$$

Para um ruído qualquer, $M^{(2)}=\mathcal{J}_{\vec{G}}$, o que é genérico demais para obtermos um resultado útil. Vamos então retomar o ruído não uniforme

$$
G_{\sigma}=\sum_{\sigma^{\prime}}\left(\eta_{\sigma^{\prime}} n_{\sigma^{\prime} \rightarrow \sigma}-\eta_{\sigma} n_{\sigma \rightarrow \sigma^{\prime}}\right)
$$

Para obter a expressão de $\mathfrak{G}^{(1)}$ substituímos 4.4 .47 na equação 4.4 .42 e usamos a definição de $\phi_{i, \sigma}$ (equação 4.4.28):

$$
\mathfrak{G}_{i, j}^{(1)}=-2 \sum_{\sigma \in \Omega} \frac{\xi_{i, \sigma}}{\lambda_{\sigma} \eta_{\Delta_{j}}^{*}}\left(\delta_{i, j}+\frac{\xi_{j, \sigma}}{\lambda_{\sigma}}\right) \sum_{k} \phi_{k, \sigma} .
$$

O jacobiano de $\vec{G}$ é, para esse ruído

$$
\left(\mathcal{J}_{\vec{G}}\right)_{\sigma, \sigma^{\prime}}=n_{\sigma^{\prime} \rightarrow \sigma}-\delta_{\sigma, \sigma^{\prime}} \sum_{\sigma^{\prime \prime}} n_{\sigma \rightarrow \sigma^{\prime \prime}}
$$

Logo, para obtermos $\mathfrak{G}^{(2)}$ calculamos primeiro $\widetilde{1}^{(i)} \cdot M_{\Delta, \Delta}^{(2)} \cdot \widetilde{\zeta}^{(j)}$

$$
\widetilde{1}^{(i)} \cdot M_{\Delta, \Delta}^{(2)} \cdot \widetilde{\zeta}^{(j)}=\sum_{\sigma \in \Delta_{i}} \sum_{\sigma^{\prime} \in \Delta_{j}} \zeta_{\sigma^{\prime}}\left(n_{\sigma^{\prime} \rightarrow \sigma}-\delta_{\sigma, \sigma^{\prime}} \sum_{\sigma^{\prime \prime}} n_{\sigma \rightarrow \sigma^{\prime \prime}}\right)=
$$




$$
\begin{gathered}
=\frac{1}{\eta_{\Delta_{j}}^{*}}\left(\sum_{\sigma \in \Delta_{i}} \sum_{\sigma^{\prime} \in \Delta_{j}} \eta_{\sigma^{\prime}}^{*} n_{\sigma^{\prime} \rightarrow \sigma}-\delta_{i, j} \sum_{\sigma^{\prime \prime}} \sum_{\sigma \in \Delta_{i}} \eta_{\sigma}^{*} n_{\sigma \rightarrow \sigma^{\prime \prime}}\right) \Rightarrow \\
\widetilde{1}^{(i)} \cdot M_{\Delta, \Delta}^{(2)} \cdot \widetilde{\zeta}^{(j)}=\frac{1}{\eta_{\Delta_{j}}^{*}}\left(\sum_{\sigma \in \Delta_{i}} \phi_{j, \sigma}-\delta_{i, j} \sum_{\sigma} \phi_{i, \sigma}\right) .
\end{gathered}
$$

A seguir, calculamos $\vec{y}^{(i)} \cdot M_{\Omega, \Delta}^{(2)} \cdot \widetilde{\zeta}^{(j)}$. Como $\sigma \neq \sigma^{\prime}$ (na expressão 4.4.49), então temos nesse caso apenas

$$
\begin{gathered}
\vec{y}^{(i)} \cdot M_{\Omega, \Delta}^{(2)} \cdot \widetilde{\zeta}^{(j)}=\sum_{\sigma \in \Omega} \sum_{\sigma^{\prime} \in \Delta_{j}} \frac{-\xi_{i, \sigma}}{\lambda} \zeta_{\sigma^{\prime}} n_{\sigma^{\prime} \rightarrow \sigma}=-\sum_{\sigma \in \Omega} \frac{\xi_{i, \sigma}}{\lambda \eta_{\Delta_{j}}^{*}} \sum_{\sigma^{\prime} \in \Delta_{j}} \eta_{\sigma^{\prime}}^{*} n_{\sigma^{\prime} \rightarrow \sigma} \Rightarrow \\
\vec{y}^{(i)} \cdot M_{\Omega, \Delta}^{(2)} \cdot \widetilde{\zeta}^{(j)}=-\sum_{\sigma \in \Omega} \frac{\xi_{i, \sigma} \phi_{j, \sigma}}{\lambda \eta_{\Delta_{j}}^{*}} .
\end{gathered}
$$

Portanto, juntando 4.4.50 e 4.4.51

$$
\mathfrak{G}_{i, j}^{(2)}=\frac{1}{\eta_{\Delta_{j}}^{*}}\left(\sum_{\sigma \in \Delta_{i}} \phi_{j, \sigma}-\sum_{\sigma \in \Omega} \frac{\xi_{i, \sigma} \phi_{j, \sigma}}{\lambda_{\sigma}}-\delta_{i, j} \sum_{\sigma} \phi_{j, \sigma}\right) .
$$

Ao contrário do acontece em $\mathfrak{G}^{(1)}$, se $\Omega=\varnothing$ a expressão para $\mathfrak{G}^{(2)}$ se simplifica, mas ainda temos $\mathfrak{G}^{(2)} \neq 0$, a saber

$$
\mathfrak{G}_{i, j}^{(2)}=\frac{1}{\eta_{\Delta_{j}}^{*}}\left(\sum_{\sigma \in \Delta_{i}} \phi_{j, \sigma}-\delta_{i, j} \sum_{\sigma} \phi_{j, \sigma}\right) .
$$

Podemos provar expressões análogas às da equação 4.4.46 para essa matriz $\mathfrak{G}^{(2)}$ :

$$
\sum_{i} \mathfrak{G}_{i, j}^{(2)}=\frac{1}{\eta_{\Delta_{j}}^{*}}\left(\sum_{i} \sum_{\sigma \in \Delta_{i}} \phi_{j, \sigma}-\sum_{\sigma \in \Omega} \phi_{j, \sigma} \sum_{i} \frac{\xi_{i, \sigma}}{\lambda_{\sigma}}-\sum_{\sigma} \phi_{j, \sigma}\right)
$$

e aplicando a equação 4.4 .43 temos

$$
\sum_{i} \mathfrak{G}_{i, j}^{(2)}=\frac{1}{\eta_{\Delta_{j}}^{*}}\left(\sum_{\sigma \in \Delta} \phi_{j, \sigma}+\sum_{\sigma \in \Omega} \phi_{j, \sigma}-\sum_{\sigma} \phi_{j, \sigma}\right)=0
$$

e

$$
\sum_{j} \mathfrak{G}_{i, j}^{(2)} \eta_{\Delta_{j}}^{*}=\sum_{j} \sum_{\sigma \in \Delta_{i}} \phi_{j, \sigma}-\sum_{\sigma \in \Omega}\left(\frac{\xi_{i, \sigma}}{\lambda_{\sigma}} \sum_{j} \phi_{j, \sigma}\right)-\sum_{\sigma} \phi_{j, \sigma}=0,
$$

pois 4.4.55 se torna equivalente à equação de robustez 4.4.30. Logo, podemos escrever 


$$
\overrightarrow{1} \cdot \mathfrak{G}^{(2)}=\overrightarrow{0} \quad \text { e } \quad \mathfrak{G}^{(2)} \cdot \vec{H}=\overrightarrow{0} .
$$

Notamos que a expressão 4.4.46, para a matriz $\mathfrak{G}^{(1)}$ vale para um ruído geral (apenas com as propriedades que exigimos no começo desta seção). Porém apenas $\overrightarrow{1} \cdot \mathfrak{G}^{(2)}=\overrightarrow{0}$ vale no caso geral (isso segue de $\overrightarrow{1} \cdot \mathcal{J}_{\vec{G}}=\overrightarrow{0}$, que por sua vez segue de $\overrightarrow{1} \cdot \vec{G}=0$, que é uma imposição necessária, para que a soma das coordenadas permaneça uma constante depois da introdução do ruído). Essas duas expressões podem, em um certo sentido, serem pensadas como análogas das expressões

$$
\overrightarrow{1} . \mathcal{J}_{\vec{F}}^{*}=\overrightarrow{0} \quad \text { e } \quad \mathcal{J}_{\vec{F}}^{*} \cdot \vec{\eta}^{*}=\overrightarrow{0},
$$

que encontramos na seção 4.2. Elas também implicam que um dos autovalores nulos sobrevive à adição de ruído. Mais uma vez, esse é o artefato de imersão, causado pela conservação da soma das variáveis. Isso segue da interpretação desses vetores $\overrightarrow{1}$ e $\vec{H}$, que estamos usando, em termos de vetores de $\mathbb{R}^{M}$. A saber, um vetor linha

$$
\left[\begin{array}{lll}
a_{1} & \ldots & a_{k}
\end{array}\right], \text { corresponde a } \sum_{i} a_{i} \vec{u}^{(i)}
$$

e um vetor coluna

$$
\left[\begin{array}{c}
b_{1} \\
\vdots \\
b_{k}
\end{array}\right] \text {, corresponde a } \sum_{i} b_{i} \vec{v}^{(i)}
$$

Logo, $\overrightarrow{1}$ corresponde a

$$
\left[\begin{array}{llll}
\overrightarrow{1}_{1} & \ldots & \overrightarrow{1}_{k} & \overrightarrow{1}_{\Omega}
\end{array}\right]
$$

(note que pelas nossas convenções esse vetor também seria denotado por $\overrightarrow{1}$ ) uma vez que as equações 4.4.35 e 4.4.43 implicam que

$$
\sum_{i} \vec{y}^{(i)}=\overrightarrow{1}_{\Omega}
$$

De forma parecida, $\vec{H}$ corresponde a

$$
\left[\begin{array}{c}
\vec{\eta}_{1}^{*} \\
\vdots \\
\vec{\eta}_{k}^{*} \\
\overrightarrow{0}_{\Omega}
\end{array}\right]=\vec{\eta}^{*} \text {, pois } \vec{\eta}_{i}^{*}=\eta_{\Delta_{i}}^{*} \vec{\zeta}_{i} \text { por definição. }
$$

Também podemos, usando um argumento semelhante ao que usamos na seção 4.2.2, provar que $\mathfrak{G}^{(1)}\left(\mathfrak{G}^{(2)}\right)$ é positiva (negativa) semi-definida e o 0 é o único autovalor com parte 
real nula, com multiplicidade algébrica 1. Porém, neste caso isso não nos permite determinar os sinais das partes reais dos autovalores que estamos perturbando, pois as derivadas deles com respeito a intensidade de ruído, são dadas pelos autovalores da matriz $\mathfrak{G}=\mathfrak{G}^{(1)}+\mathfrak{G}^{(2)}$ :

$$
\begin{gathered}
\mathfrak{G}_{i, j}=\frac{1}{\eta_{\Delta_{j}}^{*}}\left(-\delta_{i, j}\left(\sum_{\sigma} \phi_{j, \sigma}+2 \sum_{\sigma \in \Omega} \frac{\xi_{i, \sigma}}{\lambda_{\sigma}} \sum_{k} \phi_{k, \sigma}\right)+\right. \\
\left.\quad+\sum_{\sigma \in \Delta_{i}} \phi_{j, \sigma}-\sum_{\sigma \in \Omega} \frac{\xi_{i, \sigma}}{\lambda_{\sigma}}\left(\phi_{j, \sigma}+\frac{2 \xi_{j, \sigma}}{\lambda_{\sigma}} \sum_{k} \phi_{k, \sigma}\right)\right)
\end{gathered}
$$

que não é necessariamente semi-definida (ela pode ter autovalores com parte real positiva e com parte real negativa). Por completeza, no caso do ruído uniforme obtido com $n_{\sigma \rightarrow \sigma^{\prime}}=1$, que consideramos anteriormente, a matriz $\mathfrak{G}$ se torna (trocando $\phi_{i, \sigma}$ por $\eta_{\Delta_{i}}^{*}$ ):

$$
\mathfrak{G}_{i, j}=-\delta_{i, j}\left(M+2 \sum_{\sigma \in \Omega} \frac{\xi_{i, \sigma}}{\lambda_{\sigma} \eta_{\Delta_{j}}^{*}}\right)+\left|\Delta_{i}\right|-\sum_{\sigma \in \Omega} \frac{\xi_{i, \sigma}}{\lambda_{\sigma}}\left(1+\frac{2 \xi_{j, \sigma}}{\lambda_{\sigma} \eta_{\Delta_{j}}^{*}}\right) .
$$

Interessantemente, essa mesma matriz, $\mathfrak{G}$, surge em outra análise das equações 4.4.30. A equação de robustez $\overrightarrow{1}_{i} \cdot\left(\vec{G}_{i}^{*}-\mathcal{N}_{i} \cdot\left(\mathcal{J}_{\Omega}^{*}\right)^{-1} \cdot \vec{G}_{\Omega}^{*}\right)=0 \forall i$ pode primeiro ser reescrita usando $\vec{y}^{(i)}=-\overrightarrow{1}_{i} \cdot \mathcal{N}_{i} \cdot\left(\mathcal{J}_{\Omega}^{*}\right)^{-1}$ como

$$
\overrightarrow{1}_{i} \cdot \vec{G}_{i}^{*}+\vec{y}^{(i)} \cdot \vec{G}_{\Omega}^{*}=0 \forall i .
$$

Depois, podemos considerar o campo $\overrightarrow{\mathcal{G}}$ em $\operatorname{Sim}_{k}$, onde $\mathcal{G}_{i}=\overrightarrow{1}_{i} \cdot \vec{G}_{i}+\vec{y}^{(i)} \cdot \vec{G}_{\Omega}$ (note que o campo $\vec{G}$ não é avaliado no ponto fixo robusto) e o fluxo $\dot{\vec{x}}=\overrightarrow{\mathcal{G}}(\vec{x})$, de forma que os pontos robustos seriam pontos fixos desse fluxo (no sentido que as soluções da equação de robustez são os pontos fixos). Para que essa análise faça sentido, precisamos redefinir algumas coisas. Como na variedade $\mathcal{M}_{\Delta}$ as opiniões em $\Omega$ não sobrevivem, então $\eta_{\sigma}=$ $0 \forall \sigma \in \Omega$ e como estamos estudando os pontos robustos, dados os pontos de coexistência $\vec{\zeta}_{i}$ das diversas componentes de $\Delta$, na ausência de ruído, então vamos redefinir todas as funções de $\vec{\eta}$ como funções dos $\eta_{\Delta_{i}}^{*}$, efetivamente restringindo a definição dessas funções ao hiperplano $\mathcal{F}\left(\vec{\zeta}_{1}, \ldots, \vec{\zeta}_{k}\right)$ aonde estão os pontos fixos de $\mathcal{M}_{\Delta}$ para o modelo sem ruído. Mais precisamente, se temos uma função $f(\vec{\eta})$, iremos reescrevê-la como uma função $f^{\prime}$ definida por $f^{\prime}\left(\eta_{\Delta_{1}}^{*}, \ldots, \eta_{\Delta_{k}}^{*}\right)=f\left(\eta_{\Delta_{1}}^{*} \vec{\zeta}_{1}, \ldots, \eta_{\Delta_{k}}^{*} \vec{\zeta}_{k}, \overrightarrow{0}_{\Omega}\right)$. De maneira menos abstrata, para o ruído não-uniforme da equação 4.4.47, iremos considerar as seguintes modificações das definições dadas nas equações 4.4.26 e 4.4.28:

$$
\xi_{i, \sigma}(\vec{x}) \equiv x_{i}^{2} \sum_{\sigma^{\prime} \in \Delta_{i}} \zeta_{\sigma^{\prime}}^{2} p_{\sigma \rightarrow \sigma^{\prime}}, \quad \lambda_{\sigma}(\vec{x}) \equiv-\sum_{i} \xi_{i, \sigma}(\vec{x}), \quad \phi_{i, \sigma}(\vec{x}) \equiv x_{i} \sum_{\sigma^{\prime} \in \Delta_{i}} \zeta_{\sigma^{\prime}} n_{\sigma^{\prime} \rightarrow \sigma} .
$$


e o fluxo $\dot{\vec{x}}=\overrightarrow{\mathcal{G}}(\vec{x})$ onde

$$
\mathcal{G}_{i}(\vec{x}) \equiv \sum_{\sigma \in \Delta_{i}} \sum_{j} \phi_{j, \sigma}(\vec{x})-\sum_{\sigma} \phi_{i, \sigma}(\vec{x})-\sum_{\sigma \in \Omega} \frac{\xi_{i, \sigma}(\vec{x})}{\lambda_{\sigma}(\vec{x})} \sum_{j} \phi_{j, \sigma}(\vec{x}) .
$$

Voltando para o caso de um ruído geral, iremos mostrar que $\mathfrak{G}=\mathcal{J}_{\overrightarrow{\mathcal{G}}}(\vec{H})$, o jacobiano desse campo avaliado em $\vec{H}$. Para isso, primeiro suponha que $f$ é uma função de $\vec{\eta}$ que reescrevemos como uma função $f^{\prime}\left(x_{1}, \ldots, x_{k}\right)=f\left(x_{1} \vec{\zeta}_{1}, \ldots, x_{k} \vec{\zeta}_{k}, \overrightarrow{0}_{\Omega}\right)$, logo, pela rega da cadeia,

$$
\frac{\partial f^{\prime}}{\partial x_{i}}=\sum_{\sigma \in \Delta_{i}} \zeta_{\sigma} \frac{\partial f}{\partial \eta_{\sigma}}
$$

onde $\partial f / \partial \eta_{\sigma}$ também foi reescrita como uma função de $x_{1}, \ldots, x_{k}$. Portanto

$$
\frac{\partial f^{\prime}}{\partial x_{i}}=(\vec{\nabla} f)_{i} \cdot \vec{\zeta}_{i}=(\vec{\nabla} f)_{\Delta} \cdot \widetilde{\zeta}_{i}
$$

Também vamos precisar da expressão para as derivadas de $\xi_{i, \sigma}(\vec{x}) / \lambda_{\sigma}(\vec{x})$.

$$
\frac{\partial}{\partial x_{j}} \frac{\xi_{i, \sigma}(\vec{x})}{\lambda_{\sigma}(\vec{x})}=\frac{1}{\lambda_{\sigma}^{2}(\vec{x})}\left(\frac{\partial \xi_{i, \sigma}(\vec{x})}{\partial x_{j}} \lambda_{\sigma}(\vec{x})-\frac{\partial \lambda_{\sigma}(\vec{x})}{\partial x_{j}} \xi_{i, \sigma}(\vec{x})\right)
$$

Lembrando que $\xi_{i, \sigma}(\vec{x})=a_{i, \sigma} \cdot x_{i}^{2}$ para alguma constante $a_{i, \sigma}$ e que a equação 4.4 .59 expressa $\lambda_{\sigma}(\vec{x})$ em termos de $\xi_{i, \sigma}(\vec{x})$, temos

$$
\begin{gathered}
\frac{1}{\lambda_{\sigma}^{2}(\vec{x})}\left(\frac{2 \delta_{i, j} \xi_{i, \sigma}(\vec{x})}{x_{j}} \lambda_{\sigma}(\vec{x})+\frac{2 \xi_{j, \sigma}(\vec{x})}{x_{j}} \xi_{i, \sigma}(\vec{x})\right) \Rightarrow \\
\frac{\partial}{\partial x_{j}} \frac{\xi_{i, \sigma}(\vec{x})}{\lambda_{\sigma}(\vec{x})}=\frac{2 \xi_{i, \sigma}(\vec{x})}{x_{j} \lambda_{\sigma}(\vec{x})}\left(\delta_{i, j}+\frac{\xi_{j, \sigma}(\vec{x})}{\lambda_{\sigma}(\vec{x})}\right) .
\end{gathered}
$$

No caso geral $\mathcal{G}_{i}=\overrightarrow{1}_{i} \cdot \vec{G}_{i}+\vec{y}^{(i)}(\vec{x}) \cdot \vec{G}_{\Omega}=\widetilde{1}_{i} \cdot \vec{G}_{\Delta}+\vec{y}^{(i)}(\vec{x}) \cdot \vec{G}_{\Omega}$, onde lembramos que as coordenadas de $\vec{y}^{(i)}(\vec{x})$ são $-\xi_{i, \sigma}(\vec{x}) / \lambda_{\sigma}(\vec{x})$. Portanto

$$
\frac{\partial \mathcal{G}_{i}}{\partial x_{j}}=\widetilde{1}_{i} \cdot \frac{\partial \vec{G}_{\Delta}}{\partial x_{j}}+\frac{\partial \vec{y}^{(i)}(\vec{x})}{\partial x_{j}} \cdot \vec{G}_{\Omega}+\vec{y}^{(i)}(\vec{x}) \cdot \frac{\partial \vec{G}_{\Omega}}{\partial x_{j}}
$$

Segue da equação 4.4.61 que

$$
\frac{\partial \mathcal{G}_{i}}{\partial x_{j}}=\widetilde{1}_{i} \cdot\left(\mathcal{J}_{\vec{G}}\right)_{\Delta, \Delta} \cdot \widetilde{\zeta}_{j}+\vec{y}^{(i)}(\vec{x}) \cdot\left(\mathcal{J}_{\vec{G}}\right)_{\Omega, \Delta} \cdot \widetilde{\zeta}_{j}-\sum_{\sigma \in \Omega} G_{\sigma} \frac{\partial}{\partial x_{j}} \frac{\xi_{i, \sigma}(\vec{x})}{\lambda_{\sigma}(\vec{x})}
$$


Usando então a equação 4.4 .62 e a definição das matrizes $M^{(2)}$ e $\mathfrak{G}^{(2)}$, adaptadas como funções de $\vec{x}$ e a expressão para $\mathfrak{G}^{(1)}$ da equação 4.4 .42 (que não tem dependências explícitas com as coordenadas de $\vec{\psi}$ ), adaptada da mesma forma, então

$$
\frac{\partial \mathcal{G}_{i}}{\partial x_{j}}=\vec{u}^{(i)}(\vec{x}) \cdot M^{(2)} \cdot \vec{v}^{(j)}(\vec{x})-\sum_{\sigma \in \Omega} \frac{2 \xi_{i, \sigma}(\vec{x}) G_{\sigma}}{x_{j} \lambda_{\sigma}(\vec{x})}\left(\delta_{i, j}+\frac{\xi_{j, \sigma}(\vec{x})}{\lambda_{\sigma}(\vec{x})}\right)=\mathfrak{G}_{i, j}^{(2)}(\vec{x})+\mathfrak{G}_{i, j}^{(1)}(\vec{x}),
$$

de forma que

$$
\mathcal{J}_{\overrightarrow{\mathcal{G}}}(\vec{H})=\mathfrak{G}
$$

Para tornar isso mais concreto, vamos repetir esse desenvolvimento no caso do ruído nãouniforme. Como

$$
\begin{gathered}
\frac{\partial \phi_{i, \sigma}(\vec{x})}{\partial x_{j}}=\delta_{i, j} \frac{\phi_{i, \sigma}}{x_{j}}, \text { então } \\
\frac{\partial \mathcal{G}_{i}(\vec{x})}{\partial x_{j}}=\sum_{\sigma \in \Delta_{i}} \sum_{k} \frac{\partial \phi_{k, \sigma}(\vec{x})}{\partial x_{j}}-\sum_{\sigma} \frac{\partial \phi_{i, \sigma}(\vec{x})}{\partial x_{j}}-\sum_{\sigma \in \Omega} \frac{\partial}{\partial x_{j}}\left(\frac{\xi_{i, \sigma}(\vec{x})}{\lambda_{\sigma}(\vec{x})} \sum_{k} \phi_{k, \sigma}(\vec{x})\right)= \\
=\sum_{\sigma \in \Delta_{i}} \frac{\phi_{j, \sigma}(\vec{x})}{x_{j}}-\delta_{i, j} \sum_{\sigma} \frac{\phi_{j, \sigma}(\vec{x})}{x_{j}}-\sum_{\sigma \in \Omega}\left(\frac{\xi_{i, \sigma}(\vec{x}) \phi_{j, \sigma}(\vec{x})}{\lambda_{\sigma}(\vec{x}) x_{j}}+\right. \\
\left.+\frac{\partial}{\partial x_{j}}\left(\frac{\xi_{i, \sigma}(\vec{x})}{\lambda_{\sigma}(\vec{x})}\right) \sum_{k} \phi_{k, \sigma}(\vec{x})\right)
\end{gathered}
$$

e usando a equação 4.4 .62 temos

$$
\begin{gathered}
\frac{1}{x_{j}}\left(\sum_{\sigma \in \Delta_{i}} \phi_{j, \sigma}(\vec{x})-\delta_{i, j} \sum_{\sigma} \phi_{j, \sigma}(\vec{x})-\sum_{\sigma \in \Omega}\left(\frac{\xi_{i, \sigma}(\vec{x}) \phi_{j, \sigma}(\vec{x})}{\lambda_{\sigma}(\vec{x})}+\right.\right. \\
\left.\left.+\sum_{k} \frac{2 \phi_{k, \sigma}(\vec{x}) \xi_{i, \sigma}(\vec{x})}{\lambda_{\sigma}(\vec{x})}\left(\delta_{i, j}+\frac{\xi_{j, \sigma}(\vec{x})}{\lambda_{\sigma}(\vec{x})}\right)\right)\right)
\end{gathered}
$$

e reorganizando os termos

$$
\begin{aligned}
& \frac{\partial \mathcal{G}_{i}(\vec{x})}{\partial x_{j}}=\frac{1}{x_{j}}\left(-\delta_{i, j}\left(\sum_{\sigma} \phi_{j, \sigma}(\vec{x})+2 \sum_{\sigma \in \Omega} \frac{\xi_{i, \sigma}(\vec{x})}{\lambda_{\sigma}(\vec{x})} \sum_{k} \phi_{k, \sigma}(\vec{x})\right)+\right. \\
& \left.+\sum_{\sigma \in \Delta_{i}} \phi_{j, \sigma}(\vec{x})-\sum_{\sigma \in \Omega} \frac{\xi_{i, \sigma}(\vec{x})}{\lambda_{\sigma}(\vec{x})}\left(\phi_{j, \sigma}(\vec{x})+\frac{2 \xi_{j, \sigma}(\vec{x})}{\lambda_{\sigma}(\vec{x})} \sum_{k} \phi_{k, \sigma}(\vec{x})\right)\right) .
\end{aligned}
$$

Logo, comparando com a equação 4.4 .42 chegamos a conclusão que 


$$
\frac{\partial \mathcal{G}_{i}}{\partial x_{j}}(\vec{H})=\mathfrak{G}_{i, j}(\vec{x}) \Rightarrow \mathcal{J}_{\overrightarrow{\mathcal{G}}}(\vec{H})=\mathfrak{G}
$$

A conexão exata entre esse fluxo $\dot{\vec{x}}=\overrightarrow{\mathcal{G}}$ e a dinâmica, após a adição de ruído, na variedade central do modelo sem ruído, que é o hiperplano $\mathcal{F}\left(\vec{\zeta}_{1}, \ldots, \overrightarrow{\zeta_{k}}\right)$, ainda não está clara para nós. Porém, esse fluxo tem outra propriedade interessante, quando usamos o ruído não-uniforme $G_{\sigma}=\sum_{\sigma^{\prime}}\left(\eta_{\sigma^{\prime}} n_{\sigma^{\prime} \rightarrow \sigma}-\eta_{\sigma} n_{\sigma \rightarrow \sigma^{\prime}}\right)$. Nesse caso, a nossa hipótese que $G_{\sigma}>0$, sempre que $\eta_{\sigma}=0$, é equivalente a $n_{\sigma \rightarrow \sigma^{\prime}}>0$, para todo $\sigma$ e $\sigma^{\prime}$. Por outro lado, isso implica que $\phi_{i, \sigma}(\vec{x})>0$, sempre que $x_{i}>0$ e $\operatorname{logo} \sum_{i} \phi_{i, \sigma}(\vec{x})>0 \forall \vec{x} \in \operatorname{Sim}_{k} . x_{i}=0$ implica que

$$
\mathcal{G}_{i}(\vec{x})=\sum_{\sigma \in \Delta_{i}} \sum_{j} \phi_{j, \sigma}(\vec{x})>0
$$

e logo o campo $-\overrightarrow{\mathcal{G}}$ aponta para fora do espaço de fase, ao longo de toda a borda. Relembrando o teorema de Poincaré-Hopf, que usamos na seção 4.3, devemos ter 2 possibilidades:

- Os pontos fixos de $\overrightarrow{\mathcal{G}}$ no interior de $\operatorname{Sim}_{k}$ não são isolados (pelo teorema da função implícita, isso acontece somente se o 0 tiver multiplicidade maior que 1 , para $\mathfrak{G}$, avaliada em algum ponto fixo).

- Esses pontos fixos são isolados e logo podemos aplicar o teorema de Poincaré-Hopf, que nos diz que a soma dos índices dos pontos fixos de $-\overrightarrow{\mathcal{G}}$ é a característica de Euler de $\operatorname{Sim}_{k}$, que é $1\left(\log\right.$, a soma dá $(-1)^{k-1}$ para $\left.\overrightarrow{\mathcal{G}}\right)$.

De qualquer forma, deve existir algum ponto fixo do fluxo tal que

$$
x_{i}>0 \forall i \quad \text { e } \quad \sum_{i} x_{i}=1,
$$

sejam eles isolados ou não. Portanto, pelo menos um ponto fixo de $\mathcal{M}_{\Delta}$ sobrevive à adição do ruído infinitesimal (é robusto), se essa variedade for atrativa na vizinhança de seus pontos fixos, para o caso sem ruído (note que essa é uma condição necessária para as equações 4.4.30 serem válidas). Também podemos argumentar que deve existir uma variedade atrativa para o fluxo $\dot{\vec{x}}=\overrightarrow{\mathcal{G}}$ (que pode não ser um ponto fixo), embora o significado de tal variedade para as trajetórias no espaço de fase em que os $\eta_{\sigma}$ são as variáveis, também não esteja claro. Por outro lado, se provarmos para um dada regra e um dado ruído que o jacobiano de $\overrightarrow{\mathcal{G}}$ nos pontos fixos deve ser negativo semi-definido, com o autovalor 0 tendo multiplicidade 1 (e nenhum outro autovalor com parte real nula), então poderiamos mostrar com um argumento parecido com o da seção 4.3 que somente um ponto fixo sobrevive. Dada essa conexão entre $\mathcal{J}_{\overrightarrow{\mathcal{G}}}$ e $\mathfrak{G}$, é tentador conjecturar que $\mathfrak{G}$ é negativa semi-definida (com o 0 como único autovalor nulo e com multiplicidade 1) em todos os pontos fixos. Se isso fosse verdade, teríamos ao mesmo tempo uma prova da unicidade dos pontos fixos que sobrevivem à adição do ruído infinitesimal e um retrato completo da estabilidade das trajetórias em torno desses 


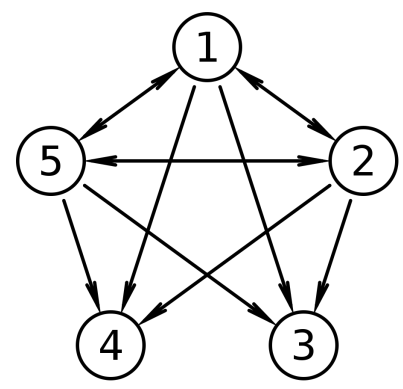

Figura 4.4.1. Contra-exemplo, em que $\mathfrak{G}$ não é negativa semidefinida para todos os pontos fixos com $\Delta=\{3,4\}$ e ruído uniforme (o que segue pelo fato que mais de um ponto fixo sobrevive à adição do ruído)

pontos robustos. Também significaria que $\vec{H}$ poderia ser calculado simplesmente integrando numericamente as equações

$$
\dot{x}_{i}=\mathcal{G}_{i}(\vec{x}) .
$$

Finalmente, como $\mathfrak{G}^{(1)}=0$ se $\Omega=\varnothing$, então $\mathfrak{G}$ é sempre negativa semi-definida nesse caso.

Porém, existem contra-exemplos em que $\mathfrak{G}$ tem autovalores com parte real positiva, a saber, na regra da figura 4.4.1, se tomarmos todos os pesos como sendo 0 ou 1 , ruído uniforme e analisarmos os pontos fixos de $\mathcal{M}_{\{3,4\}}$ que sobrevivem à adição de ruído, encontramos 3 pontos fixos diferentes ( 3 soluções para as equações 4.4.30), ao invés de um único, que seria esperado se a conjectura fosse verdadeira (mais precisamente, temos 2 atratores, em que a simetria entre 3 e 4 é quebrada e uma sela, no ponto em que $\eta_{3}=\eta_{4}$ ).

Independentemente disso, podemos estabelecer que sempre existe algum ponto fixo robusto em $\mathcal{M}_{\Delta}$ e que o fluxo $\dot{\vec{x}}=\overrightarrow{\mathcal{G}}$ sempre tem um atrator, o que talvez indique que sempre algum atrator sobreviva. Além disso, no apêndice A.1, nós provamos que para uma regra sem atratores, devem existir ciclos heteroclínicos (pelo menos um ciclo). Os nossos resultados mostram que mesmo a adição de um ruído infinitesimal destrói todos os pontos fixos que fazem parte desses ciclos heteroclínicos (pois todos esses pontos envolvem uma opinião $\sigma$ que não sobrevive e tal que $\lambda_{\sigma}=0$ ), o que implica que o modelo deve exibir ou ciclos limites, ou quasiperiodicidade, ou até mesmo comportamento caótico, nesses casos.

\subsection{Generalizações do modelo Sznajd}

O tratamento do modelo Sznajd na aproximação de campo médio pode ser facilmente extendido para o caso em que é necessário formar um grupo de tamanho $q \geq 2$ de sítios que concordem entre si, antes que o convencimento seja possível. E em que cada grupo tenta convencer $r$ sítios. Nesse caso, a expressão para $F_{\sigma}$ passa a ser 


$$
\dot{\eta}_{\sigma}=F_{\sigma}=r \sum_{\sigma^{\prime}} \eta_{\sigma} \eta_{\sigma^{\prime}}\left(\eta_{\sigma}^{q-1} p_{\sigma^{\prime} \rightarrow \sigma}-\eta_{\sigma^{\prime}}^{q-1} p_{\sigma \rightarrow \sigma^{\prime}}\right)
$$

Para obter essa equação é necessário fazer uma contabilidade de todos os processos que mudam o estado, desconsiderando correlações espaciais. Vamos assumir, logo de início, que estamos fazendo a simulação em um grafo completo e que a probabilidade de cada sítio ter uma dada opinião depende apenas da proporção em que essa opinião aparece na rede (independentemente do estado da rede), de forma que o formalismo completo do capítulo 3 não será necessário. Suponha primeiro que já tenhamos um grupo de tamanho $q$, em que todos tem opinião $\sigma$. Precisamos então sortear um sítio e o grupo "irá tentar convencer" esse sítio sorteado. Seja então $B_{\sigma}$, a probabilidade de sortear um sítio aleatoriamente e conseguir convencê-lo para a opinião $\sigma$ (usando uma regra de confiança dada). Temos

$$
B_{\sigma}=\sum_{\sigma^{\prime}} \eta_{\sigma^{\prime}} p_{\sigma^{\prime} \rightarrow \sigma}
$$

e com probabilidade $1-B_{\sigma}$, nada acontece (o grupo não consegue um convencimento). Por outro lado, a probabilidade de formar um grupo com $q$ sítios que tenham opinião $\sigma$ é $\eta_{\sigma}^{q} \mathrm{e}$ dado que eles tentam convencer $r$ sítios, o número médio de sítios que esse grupo consegue convencer (descartando a possibilidade de um mesmo sítio ser convencido mais de uma vez) é

$$
\sum_{s=1}^{r} s\left(\begin{array}{l}
r \\
s
\end{array}\right) B_{\sigma}^{s}\left(1-B_{\sigma}\right)^{r-s}=B_{\sigma} r \sum_{s=0}^{r-1}\left(\begin{array}{c}
r-1 \\
s
\end{array}\right) B_{\sigma}^{s}\left(1-B_{\sigma}\right)^{r-1-s}=B_{\sigma} r
$$

de forma que o número médio de sítios que são convencidos para a opinião $\sigma$, a cada iteração, é

$$
\eta_{\sigma}^{q} B_{\sigma} r=r \sum_{\sigma^{\prime}} \eta_{\sigma^{\prime}} \eta_{\sigma}^{q} p_{\sigma^{\prime} \rightarrow \sigma}
$$

De forma análoga, se $C_{\sigma, \sigma^{\prime}}$ for a probabilidade que um sítio com opinião $\sigma$ seja convencido para a opinião $\sigma^{\prime}$, por um grupo de $q$ agentes com opinião $\sigma^{\prime}$, então

$$
C_{\sigma, \sigma^{\prime}}=\eta_{\sigma} p_{\sigma \rightarrow \sigma^{\prime}}
$$

e o número médio de sítios com opinião $\sigma$ que é convencido por um grupo de $q$ sítios com opinião $\sigma^{\prime}$ é

$$
\sum_{s=1}^{r} s\left(\begin{array}{l}
r \\
s
\end{array}\right) C_{\sigma, \sigma^{\prime}}^{s}\left(1-C_{\sigma, \sigma^{\prime}}\right)^{r-s}=C_{\sigma, \sigma^{\prime}} r .
$$

Multiplicando pela probabilidade de formar um grupo de $q$ sítios com opinião $\sigma^{\prime}$ e somando sobre todos os $\sigma^{\prime}$ possíveis segue que 


$$
\sum_{\sigma^{\prime}} \eta_{\sigma^{\prime}}^{q} C_{\sigma, \sigma^{\prime}} r=r \sum_{\sigma^{\prime}} \eta_{\sigma} \eta_{\sigma^{\prime}}^{q} p_{\sigma \rightarrow \sigma^{\prime}}
$$

é o número médio de sítios com opinião $\sigma$ que são convencidos a cada iteração. Logo, se $\eta_{\sigma}$ for o valor esperado para a proporção de sítios com opinião $\sigma$ na rede, a cada passo de tempo temos

$$
\Delta \eta_{\sigma}=\frac{1}{N} r \sum_{\sigma^{\prime}} \eta_{\sigma} \eta_{\sigma^{\prime}}\left(\eta_{\sigma}^{q-1} p_{\sigma^{\prime} \rightarrow \sigma}-\eta_{\sigma^{\prime}}^{q-1} p_{\sigma \rightarrow \sigma^{\prime}}\right)+\mathcal{O}\left(\frac{1}{N^{2}}\right) .
$$

Note que tanto a equação 4.5.2, quanto a equação 4.5.3 contabilizam o caso $\sigma=\sigma^{\prime}$, de forma que ele se cancela na expressão 4.5.4. A variação $\Delta \eta_{\sigma}$ é então o aumento médio no número de sítios com opinião $\sigma$, vezes a variação em $\eta_{\sigma}$, causada pelo aumento do número de sítios com essa opinião em uma unidade $(1 / N)$. O termo $\mathcal{O}\left(1 / N^{2}\right)$ contabiliza erros de aproximação, causados por não estarmos levando em conta repetições na hora da escolha dos sítios (por exemplo, o mesmo sítio pode ser escolhido mais de uma vez para ser convencido). No limite de redes grandes a equação 4.5.4 se torna a equação 4.5.1 (pois ela é essencialmente a equação 4.5.1 integrada pelo método de Euler, com passo $1 / N$ ). Ao invés de fazer a análise detalhada dessas equações, mostramos rapidamente o que se modifica com relação ao tratamento das seções 4.2 e 4.3 (não faremos o tratamento do ruído).

As equações para os pontos fixos passam a ser

$$
\eta_{\sigma}=0 \quad \text { ou } \quad \sum_{\sigma^{\prime}} \eta_{\sigma^{\prime}}\left(\eta_{\sigma}^{q-1} p_{\sigma^{\prime} \rightarrow \sigma}-\eta_{\sigma^{\prime}}^{q-1} p_{\sigma \rightarrow \sigma^{\prime}}\right)=0 \quad \forall \sigma .
$$

Podemos novamente pensar nos pontos fixos em termos dos conjuntos $\Delta$ e $\Omega$ de opiniões que sobrevivem e que são extintas nele. Se $\mathcal{R}_{\Delta}$ tiver mais de uma componente, onde $\mathcal{R}$ é a regra que estamos usando, os resultados finais da seção 4.2.1 seguem sem alterações (através de um raciocínio bem semelhante), ou seja, se $\vec{\zeta}_{i}$ for um ponto fixo de modelo com a regra $\mathcal{R}_{\Delta_{i}}$, onde $\Delta_{1}, \ldots, \Delta_{k}$ são as componentes de $\mathcal{R}_{\Delta}$, então o hiperplano $\left(\vec{\zeta}_{1}, \ldots, \vec{\zeta}_{k}\right)$, definido da mesma forma que na equação 4.2.13, novamente será constituído de pontos fixos. No caso geral, o jacobiano pode novamente ser decomposto como

$$
\mathcal{J}^{*}=\left[\begin{array}{cc}
\mathcal{J}_{\Delta}^{*} & \mathcal{N} \\
0 & \mathcal{J}_{\Omega}^{*}
\end{array}\right]
$$

onde a matriz $\mathcal{J}_{\Omega}^{*}$ é diagonal e seus autovalores são

$$
\lambda_{\sigma}=-\sum_{\sigma^{\prime}} \eta_{\sigma^{\prime}}^{* q} p_{\sigma \rightarrow \sigma^{\prime}}
$$

No caso em que todas as opiniões sobrevivem e o modelo tem uma componente, pode-se novamente montar uma matriz $A=\mathcal{J}^{*} D+\left(\mathcal{J}^{*} D\right)^{T}$, com $D=\operatorname{diag}\left(\vec{\eta}^{*}\right)$, que será tal que 


$$
A_{\sigma, \sigma^{\prime}}=(1-q) r \eta_{\sigma}^{*} \eta_{\sigma^{\prime}}^{*}\left(\eta_{\sigma}^{* q-1} p_{\sigma^{\prime} \rightarrow \sigma}+\eta_{\sigma^{\prime}}^{* q-1} p_{\sigma \rightarrow \sigma^{\prime}}\right) .
$$

Para todo $q \geq 2$, essa matriz satisfaz todas as propriedades qualitativas que usamos na seção 4.2.2 para deduzir as propriedades do espectro no caso do modelo Sznajd, de forma que as mesmas conclusões seguem, ou seja $\mathcal{J}_{\Delta}^{*}$ é positiva semidefinida e o 0 é o único autovalor com parte real nula, com multiplicidade algébrica e geométrica iguais a 1 e extendendo para o caso em que $\mathcal{R}_{\Delta}$ tem mais de uma componente, ambas as multiplicidades do 0 passam a ser o número de componentes $k$ de $R_{\Delta}$.

No caso em que opiniões são extintas, temos as mesmas condições em $\Delta$ e $\Omega$ para que uma opinião $\sigma \in \Omega$ tenha $\lambda_{\sigma}=0$ ou não. Se $\sigma$ é tal que $\lambda_{\sigma}=0$, temos uma equação análoga a equação 4.2.22, dada pela $q$-ésima ordem da expansão em Taylor da equação 4.5.1:

$$
\dot{\eta}_{\sigma} \simeq \mu_{\sigma} \eta_{\sigma}^{q} \text {, onde } \mu_{\sigma} \equiv r \sum_{\sigma^{\prime}} \eta_{\sigma^{\prime}}^{*} p_{\sigma^{\prime} \rightarrow \sigma}
$$

que tem solução

$$
\eta_{\sigma}(t)=\frac{\eta_{\sigma \mathrm{o}}}{\sqrt[q-1]{1-(q-1) \mu_{\sigma} \eta_{\sigma \mathrm{o}}^{q-1} t}},
$$

o que implica que as trajetórias são repelidas de $\eta_{\sigma}=0$ nesse caso também. Finalmente, se $\lambda_{\sigma}=0$ e $\mu_{\sigma}=0$ para algum $\sigma$, podemos definir $\omega$ e $\Lambda$ da mesma forma que na seção 4.2.3, para chegar nas seguintes desigualdades

$$
\begin{gathered}
-r \sum_{\sigma \in \omega} \sum_{\sigma^{\prime} \in \Omega-\omega} \eta_{\sigma^{\prime} \mathrm{o}}^{q} e^{q \lambda_{\sigma^{\prime}} t} \eta_{\sigma} p_{\sigma \rightarrow \sigma^{\prime}} \leq \dot{\eta}_{\omega} \leq r \sum_{\sigma \in \omega} \sum_{\sigma^{\prime} \in \Omega-\omega} \eta_{\sigma^{\prime} \mathrm{o}} e^{\lambda_{\sigma^{\prime}} t} \eta_{\sigma}^{q} p_{\sigma^{\prime} \rightarrow \sigma} \Rightarrow \\
-r \sum_{\sigma \in \omega} \sum_{\sigma^{\prime} \in \Omega-\omega} e^{q \lambda_{\sigma^{\prime}} t} \eta_{\sigma} \leq \dot{\eta}_{\omega} \leq r \sum_{\sigma \in \omega} \sum_{\sigma^{\prime} \in \Omega-\omega} e^{\lambda_{\sigma^{\prime}} t} \eta_{\sigma} \Rightarrow \\
-r \sum_{\sigma \in \omega} \sum_{\sigma^{\prime} \in \Omega-\omega} e^{q \Lambda t} \eta_{\sigma} \leq \dot{\eta}_{\omega} \leq r \sum_{\sigma \in \omega} \sum_{\sigma^{\prime} \in \Omega-\omega} e^{\Lambda t} \eta_{\sigma} \Rightarrow \\
-r|\Omega-\omega| e^{q \Lambda t} \eta_{\omega} \leq \dot{\eta}_{\omega} \leq r|\Omega-\omega| e^{\Lambda t} \eta_{\omega} \Rightarrow \\
-r|\Omega-\omega| e^{q \Lambda t} \leq \frac{\mathrm{d}}{\mathrm{dt}} \ln \left(\eta_{\omega}\right) \leq r|\Omega-\omega| e^{\Lambda t} .
\end{gathered}
$$

Integrando no tempo e tomando o limite $t \rightarrow \infty$ vem

$$
\eta_{\omega \mathrm{o}} e^{r|\Omega-\omega| / q \Lambda} \leq \eta_{\omega} \leq \eta_{\omega_{\mathrm{O}}} e^{-r|\Omega-\omega| / \Lambda} .
$$

Segue que as propriedades de estabilidade dos pontos fixos para $q>2$ dependem das propriedades do esqueleto da regra de confiança, exatamente da mesma forma que o caso $q=2$ depende. 
Para estudar a existência dos pontos fixos, novamente começamos com uma regra que tem esqueleto direcionado completo e definindo novamente

$$
p_{\text {min }}=\min _{\sigma \neq \sigma^{\prime}}\left\{p_{\sigma \rightarrow \sigma^{\prime}}\right\} \quad \text { e } \quad p_{\max }=\max _{\sigma \neq \sigma^{\prime}}\left\{p_{\sigma \rightarrow \sigma^{\prime}}\right\}
$$

chegamos em um análogo da desigualdade 4.3.3:

$$
F_{\sigma} \leq \epsilon^{q} p_{\max }-\epsilon p_{\min } \min _{\vec{\eta} \in \operatorname{Sim}_{M}}\left\{\sum_{\sigma^{\prime}} \eta_{\sigma^{\prime}}^{2}\right\}
$$

e usando a equação 4.3.4, chegamos em um análogo da desigualdade 4.3.5

$$
F_{\sigma} \leq \epsilon^{q} p_{\max }-\epsilon \frac{p_{\min }}{M^{q-1}}
$$

Logo se

$$
\epsilon^{q} p_{\text {max }}-\epsilon \frac{p_{\text {min }}}{M^{q-1}}<0 \Leftrightarrow \epsilon<\frac{1}{M}\left(\frac{p_{\text {min }}}{p_{\text {max }}}\right)^{1 / q-1}
$$

então $F_{\sigma}<0$. Podemos então usar o teorema de Poincaré-Hopf de maneira semelhante ao que fizemos na seção 4.3, para chegar a conclusão que existe exatamente um ponto fixo em que todas as opiniões coexistem, cujas coordenadas obedecem a

$$
\eta_{\sigma} \geq \frac{1}{M}\left(\frac{p_{\min }}{p_{\max }}\right)^{1 / q-1}
$$

Podemos repetir os argumentos de continuidade da seção 4.3 (envolvendo as regras de confiança $\mathcal{R}(\tau)$ ) para chegar a conclusão que se uma regra $\mathcal{R}$ não exibe pontos de coexistência, então existe um conjunto $\Delta \neq V(\mathcal{R})$ de opiniões, que é uma fonte em $\mathcal{R}$ e logo se $\mathcal{R}$ só tiver uma componente, ela não pode ser fortemente conexa. Se, por outro lado, $\mathcal{R}$ só tiver uma componente e ela não for fortemente conexa, então existe um sorvedouro $\Delta \neq V(\mathcal{R}) \mathrm{e}$ definindo

$$
\eta_{\Delta}=\sum_{\sigma \in \Delta} \eta_{\sigma}
$$

chegamos a conclusão que $\dot{\eta}_{\Delta}>0$ em todo o interior do espaço de fase. Extendendo para o caso em que temos muitas opiniões, chegamos no mesmo resultado que obtivemos para $q=2$, ou seja, existem pontos fixos de coexistência em que as opiniões em $\Delta$ sobrevivem se e somente se $\mathcal{R}_{\Delta}$ for uma união de componentes fortemente conexas e se $\mathcal{R}_{\Delta}$ tiver $k$ componentes, esses pontos fixos formam um hiperplano com $k-1$ dimensões. 


\subsection{O modelo do votante}

A equação de campo médio do modelo do votante com regras de confiança é (conforme deduzido na seção 3.3)

$$
\dot{\eta}_{\sigma}=\sum_{\sigma^{\prime}} \eta_{\sigma} \eta_{\sigma^{\prime}}\left(p_{\sigma^{\prime} \rightarrow \sigma}-p_{\sigma \rightarrow \sigma^{\prime}}\right)=\eta_{\sigma} \sum_{\sigma^{\prime}} T_{\sigma, \sigma^{\prime}} \eta_{\sigma^{\prime}}
$$

onde a matriz antissimétrica $T$ é definida por $T_{\sigma, \sigma^{\prime}}=p_{\sigma^{\prime} \rightarrow \sigma}-p_{\sigma \rightarrow \sigma^{\prime}}$. Essa é a mesma equação que obtivemos na seção anterior para $q=r=1$ (equação 4.5.1. Pela descrição que demos, esses parâmetros corresponderiam na verdade ao modelo Ochrombel). Porém, como $q=1$ implica que $A=0$ (equação 4.5.8), então não podemos fazer a mesma análise nesse caso. As propriedades dos pontos fixos dependem inteiramente dessa matriz $T$. Se $\vec{\eta}^{*}$ é um ponto fixo do modelo, podemos novamente definir os conjuntos $\Delta$ e $\Omega$ de opiniões que sobrevivem e que são extintas respectivamente nesse ponto fixo. Definindo $T_{\Delta}$ como a submatriz principal de $T$ com índices em $\Delta$ (T $T_{\Delta, \Delta}$ na notação da seção 4.4.2) e $\vec{\eta}_{\Delta}^{*}$ como a parte do vetor $\vec{\eta}^{*}$ que contém as coordenadas em $\Delta$, então a equação para os pontos fixos se torna

$$
T_{\Delta} \cdot \vec{\eta}_{\Delta}^{*}=\overrightarrow{0}_{\Delta}
$$

Como $\vec{\eta}^{*}$ deve pertencer a $\operatorname{Sim}_{M}$, isso implica que os pontos fixos em que somente as opiniões em $\Delta$ sobrevivem são os pontos cujas coordenadas, $\vec{\eta}_{\Delta}^{*}$, formam um autovetor à direita de $T_{\Delta}$, com autovalor 0 , que seja tal que todas as coordenadas são positivas (os autovetores são normalizados, de forma que $\overrightarrow{1} \cdot \vec{\eta}^{*}=1$ ). Além disso, a análise desses pontos fixos corresponde à análise do modelo restrito às opiniões em $\Delta$ (isso pode ser verificado diretamente na equação 4.6.1). Suponha agora que $\vec{\eta}^{*}$ seja um autovetor de $T$, correspondendo a um ponto fixo $\left(\eta_{\sigma}^{*}>0 \forall \sigma\right)$ e que a multiplicidade do 0 seja $k+1$. Como é possível formar uma base ortogonal, $\left\{\vec{v}_{1}, \ldots, \vec{v}_{k}, \vec{\eta}^{*}\right\}$, com os autovetores de $T$ que tenham autovalor 0 , então definindo $\vec{u}_{i} \equiv \vec{v}_{i}-\left(\overrightarrow{1} . \vec{v}_{i}\right) \vec{\eta}^{*}$, temos uma nova base. Além disso, o hiperplano

$$
\mathcal{F}=\left\{\vec{\eta} \in \operatorname{Sim}_{M} \mid \vec{\eta}=\vec{\eta}^{*}+\sum_{i=1}^{k} \alpha_{i} \vec{u}_{i}\right\}
$$

é composto inteiramente por pontos fixos de coexistência.

Seja $\vec{\eta}^{*}$ tal que $T \cdot \vec{\eta}^{*}=\overrightarrow{0}$ e defina o vetor $\vec{L} \equiv\left(\ln \left(\eta_{1}\right), \ldots, \ln \left(\eta_{M}\right)\right)$ no interior de $\operatorname{Sim}_{M}$. Segue da equação de campo médio 4.6.1 que

$$
\begin{gathered}
\frac{\mathrm{d}}{\mathrm{dt}} \ln \left(\eta_{\sigma}\right)=\frac{\dot{\eta}_{\sigma}}{\eta_{\sigma}}=\sum_{\sigma^{\prime}} T_{\sigma, \sigma^{\prime}} \eta_{\sigma^{\prime}} \Rightarrow \dot{\vec{L}}=T \cdot \vec{\eta} \Rightarrow \vec{\eta}^{*} \cdot \dot{\vec{L}}=\vec{\eta}^{*} \cdot T \cdot \vec{\eta}= \\
=\left(T^{T} \cdot \vec{\eta}^{*}\right)^{T} \cdot \vec{\eta}=-\left(T \cdot \vec{\eta}^{*}\right)^{T} \cdot \vec{\eta}=\overrightarrow{0} \cdot \vec{\eta}=0 \Rightarrow \vec{L} \cdot \vec{\eta}^{*}=\sum_{\sigma} \eta_{\sigma}^{*} \ln \left(\eta_{\sigma}\right)=\text { cte. }
\end{gathered}
$$


Se as coordenadas de $\vec{\eta}^{*}$ forem positivas, então $\vec{L} \cdot \vec{\eta}^{*}$ diverge para $-\infty$ nas bordas do espaço de fase e $\vec{L} \cdot \vec{\eta}^{*}$ é uma função côncava. Segue que as hipersuperfícies definidas pela interssecção de $\vec{L} \cdot \vec{\eta}^{*}=$ cte. e $\operatorname{Sim}_{M}$ são fechadas e que existe um único máximo para $\vec{L} \cdot \vec{\eta}^{*}$ no espaço de fase. Esse máximo é dado por $\vec{\eta}^{*}$, o que pode ser visto facilmente usando multiplicadores de Lagrange:

$$
\begin{gathered}
Q(\vec{\eta}, \lambda)=\sum_{\sigma} \eta_{\sigma}^{*} \ln \left(\eta_{\sigma}\right)+\lambda\left(\sum_{\sigma} \eta_{\sigma}-1\right) \therefore \frac{\partial Q}{\partial \lambda}=0 \Rightarrow \overrightarrow{1} \cdot \vec{\eta}=1 \mathrm{e} \\
\frac{\partial Q}{\partial \eta_{\sigma}}=0 \Rightarrow \lambda+\frac{\eta_{\sigma}^{*}}{\eta_{\sigma}} \Rightarrow-\lambda \eta_{\sigma}=\eta_{\sigma}^{*} \Rightarrow \vec{\eta}=\vec{\eta}^{*}, \text { pois } \overrightarrow{1} \cdot \vec{\eta}=\overrightarrow{1} \cdot \vec{\eta}^{*} .
\end{gathered}
$$

Uma consequência disso é que, ao contrário do modelo Sznajd, oscilações são possíveis no modelo com campo médio mesmo sem a adição de ruído. Considere, por exemplo, um modelo com 3 opiniões: segue que, como a matriz $T$ é antissimétrica, o 0 vai ter multiplicidade algébrica e geométrica ímpar. A multiplicidade é 3 sss $T=0$ (isso é uma consequência da matriz ser sempre diagonalizável), mas nesse caso nada acontece no campo médio (é o caso do modelo do votante usual). Se a multiplicidade for 1 e tivermos um ponto fixo associado ao autovetor, então esse é o único ponto fixo no interior do espaço de fase e as regiões em que $\vec{L} \cdot \vec{\eta}^{*}$ é constante no espaço de fase são curvas fechadas. Como o espaço de fase vai ter 2 dimensões isso implica que as trajetórias oscilam em torno do ponto fixo (que dá o máximo de $\left.\vec{L} \cdot \vec{\eta}^{*}\right)$.

Em um caso com mais opiniões, em que a matriz $T$ seja não-nula, como a multiplicidade do 0 vai sempre ter a mesma paridade que $M$ (pois $T$ é real antissimétrica), essas regras de conservação restringem as trajetórias no interior do espaço de fase a hipersuperfícies com um número ímpar de dimensões (com uma única exceção, quando $T=0$ ) e se houver pelo menos um ponto fixo de coexistência, as hipersuperfícies devem ser fechadas (consequentemente, o campo médio não deve exibir "trajetórias complicadas" com menos que 5 opiniões, pois as únicas estruturas permitidas na presença de um ponto fixo de coexistência seriam ciclos). Uma peculiaridade desse resultado é que os comportamentos estruturalmente estáveis do modelo (ou seja, as situações robustas por uma perturbação dos parâmetros $p_{\sigma \rightarrow \sigma^{\prime}}$ ) dependem da paridade do número de opiniões. Se $M$ é par, a matriz $T$ tipicamente não tem o 0 como autovalor e logo não temos oscilações. Se $M$ é ímpar, por outro lado, a multiplicidade é tipicamente 1 e a existência de um ponto fixo de coexistência (e logo de oscilações) depende de um dos autovetores ter todas as componentes positivas, o que é uma propriedade robusta por perturbações ${ }^{6}$. Segue que o comportamento típico para um número par de opiniões é não exibir oscilações, porém com um número ímpar de opiniões existe uma região do espaço de parâmetros com medida não nula em que ocorrem oscilações (porém elas não ocorrem em todo o espaço de parâmetros). Por outro lado, se tivermos uma matriz $T$ com multiplicidade do 0 maior do que no caso típico, uma perturbação destrói essa propriedade,

${ }^{6}$ Em uma situação em que a multiplicidade algébrica é 1, o autovetor sempre varia de maneira contínua. 
mas só causa uma deformação contínua de $\vec{F}$, de forma que as trajetórias devem permanecer parecidas para perturbações pequenas e devemos ver oscilações no transiente características do caso com multiplicidade alta. Em particular, se o número de opiniões é par, podemos ter, em certas regiões do espaço de parâmetros, algo reminiscente de periodicidade, mesmo que esse não seja o comportamento estruturalmente estável. Em outras palavras, podemos ter uma região do espaço de parâmetros, com medida não-nula, em que temos quasiperiodicidade, mesmo se $M$ for par.

Para analisar o caso de um ponto fixo geral, considere o jacobiano $\mathcal{J}$ de $\dot{\vec{\eta}}$,

$$
\mathcal{J}_{\sigma, \sigma^{\prime}}=\eta_{\sigma} T_{\sigma, \sigma^{\prime}}+\delta_{\sigma, \sigma^{\prime}} \sum_{\sigma^{\prime \prime}} T_{\sigma, \sigma^{\prime \prime}} \eta_{\sigma^{\prime \prime}}
$$

Assim como no modelo Sznajd, temos uma simplificação quando avaliamos o jacobiano no ponto fixo,

$$
\mathcal{J}_{\sigma, \sigma^{\prime}}^{*}=\left\{\begin{array}{ll}
\eta_{\sigma}^{*} T_{\sigma, \sigma^{\prime}}, & \text { se } \sigma \in \Delta \\
\delta_{\sigma, \sigma^{\prime}} \sum_{\sigma^{\prime \prime}} T_{\sigma, \sigma^{\prime \prime}} \eta_{\sigma^{\prime \prime}}^{*}, & \text { se } \sigma \in \Omega
\end{array},\right.
$$

implicando que o jacobiano pode ser posto na seguinte forma bloco diagonal

$$
\mathcal{J}^{*}=\left[\begin{array}{cc}
\mathcal{J}_{\Delta}^{*} & \mathcal{N} \\
0 & \mathcal{J}_{\Omega}^{*}
\end{array}\right]=\left[\begin{array}{cc}
D T_{\Delta} & \mathcal{N} \\
0 & \mathcal{J}_{\Omega}^{*}
\end{array}\right]
$$

onde a matriz $D$ é a matriz diagonal formada pelas coordenadas de $\vec{\eta}_{\Delta}^{*}$. Segue que a matriz $D T_{\Delta}$ é similar a $D^{1 / 2} T_{\Delta} D^{1 / 2}$ e a matriz $D^{1 / 2}$ é real, implicando que $D^{1 / 2} T_{\Delta} D^{1 / 2}$ é antihermitiana e logo os autovalores de $\mathcal{J}^{*}$ que vem desse bloco são nulos ou imaginários puros (o que é esperado já que como mostramos o modelo pode exibir centros). $\mathcal{J}_{\Omega}^{*}$, por outro lado, é diagonal e real, de forma que para cada opinião em $\Omega$ temos um autovalor associado

$$
\lambda_{\sigma}=\sum_{\sigma^{\prime}} T_{\sigma, \sigma^{\prime}} \eta_{\sigma^{\prime}}^{*}
$$

que diz se as trajetórias são atraídas para a variedade $\eta_{\sigma}=0$ na vizinhança do ponto fixo. 



\title{
Capítulo 5
}

\section{Resultados de Simulação}

\author{
"Mathematics is an experimental science, and definitions do not come first, \\ but later on."
}

- Oliver Heaviside

"The mind loves the unknown. It loves images whose meaning is unknown, since the meaning of the mind itself is unknown."

- René Magritte

Para comparar as trajetórias de campo médio que foram estudadas no capítulo anterior com os resultados de simulação, devemos lembrar que $\eta_{\sigma}$ é o valor esperado para a proporção de sítios com opinião $\sigma$ e que as flutuações em uma realização da simulação decaem com $N$. Por causa disso, para obter resultados úteis, devemos, ou usar tamanhos grandes de rede, ou fazer médias entre várias realizações (ou ambas as abordagens juntas). Além disso, devemos nos lembrar que a condição inicial $\left(\eta_{1}, \ldots, \eta_{M}\right)$ para o campo médio corresponde, nas simulações, a sortear a opinião de um sítio com sendo $\sigma$ com probabilidade $\eta_{\sigma}$ durante a criação da condição inicial. Para explorar o espaço de fase inteiro, sempre sorteamos o $\vec{\eta}$ correspondente a condição inicial de maneira uniforme (lembrando que as médias entre várias realizações devem usar o mesmo $\vec{\eta}$ ).

Para as simulações seguintes, a menos que especificado, usamos redes Barábasi-Albert com $10^{5}$ sítios e conectividade mínima 5 (não usamos a mesma rede, porém os parâmetros usados foram sempre estes). Essas simulações são voltadas a fornecer exemplos dos resultados de campo médio do modelo Sznajd, em particular a regra para encontrar atratores e a regra que dita a existência dos pontos fixos, sublinhando alguns aspectos contra-intuitivos e algumas formas em que as simulações divergem dos resultados de campo médio. Também apresentamos alguns exemplos da teoria de perturbação que foi desenvolvida e simulações do modelo do votante. 


\subsection{Atratores e Estabilidade}

Para ilustrar os resultados sobre as propriedades de estabilidade dos pontos fixos, considere a regra $\mathcal{R}$, na figura 5.1.1 (na verdade uma família de regras de confiança). Os conjuntos maximais independentes são $\Delta=\{1,2\},\{1,5\},\{3\}$ e $\{4\}$, mas apenas $\{1,2\}$ e $\{1,5\}$ obedecem $\Delta_{-}=\bar{\Delta}$, significando que os únicos atratores estacionários são $\mathcal{M}_{\{1,2\}}$ e $\mathcal{M}_{\{1,5\}}$. Após um transiente devemos observar então uma de 2 situações, ou as únicas opiniões sobreviventes são 1 e 2 ou elas são 1 e 5 . Podemos ver isso a partir da série temporal para $\eta_{1}+\eta_{2}+\eta_{5}$ (que tende a 1 ) e $\eta_{2} \cdot \eta_{5}$ (que tende a 0 , porém com um transiente maior) (figuras 5.1.2a e 5.1.2b).

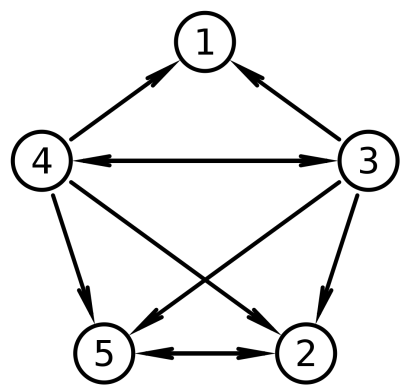

Figura 5.1.1. O esqueleto $\mathcal{R}$ de uma regra de confiança, onde 2 dos 4 conjuntos independentes maximais geram atratores. Esses atratores estáticos podem ser todos obtidos resolvendo $\Delta_{-}=\bar{\Delta}$ para essa regra, como demonstrado no capítulo 4, e são $\mathcal{M}_{\{1,2\}}$ e $\mathcal{M}_{\{1,5\}}$.

\begin{tabular}{|c|c|c|c|c|c|c|}
\hline$\Delta$ & $\bar{\Delta}$ & $\Delta_{-}$ & $\Delta_{+}$ & $i$ & $e$ & $n$ \\
\hline 3 & $1,2,4,5$ & 4 & $1,2,4,5$ & 3 & 1 & 0 \\
\hline 4 & $1,2,3,5$ & 3 & $1,2,3,5$ & 3 & 1 & 0 \\
\hline 3,4 & $1,2,5$ & 3,4 & $1,2,3,4,5$ & 4 & 0 & 0 \\
\hline $1 \times 2,5$ & 3,4 & $2,3,4,5$ & 2,5 & 1 & 2 & 1 \\
\hline
\end{tabular}

Tabela 5.1.1. Os pontos fixos da regra da figura 5.1.1 que não fazem parte de atratores, denotados pelas opiniões que sobrevivem neles $(\Delta)$. A linha de pontos fixos conectando a aresta $\overline{P_{2} P_{5}}$ ao vértice $P_{1}$ está denotada por $1 \times 2,5$. Para cada ponto fixo o número de direções instáveis, estáveis e neutras ( $i, e, n$ respectivamente). Esses valores podem ser calculados usando as fórmulas obtidas no capítulo 4.

Os outros pontos fixos podem ser encontrados olhando para os subgrafos induzidos da regra que são uniões de componentes fortemente conexas. Eles são, $\mathcal{R}_{\{3\}}, \mathcal{R}_{\{4\}}, \mathcal{R}_{\{3,4\}}$ e $\mathcal{R}_{\{1,2,5\}}$. Note que $\mathcal{R}_{\{1,2,5\}}=\mathcal{R}_{\{1\}} \cup \mathcal{R}_{\{2,5\}}$ e que ambas as componentes são fortemente conexas. Isso significa que nós vamos ter, na verdade, uma linha de pontos fixos conectando 


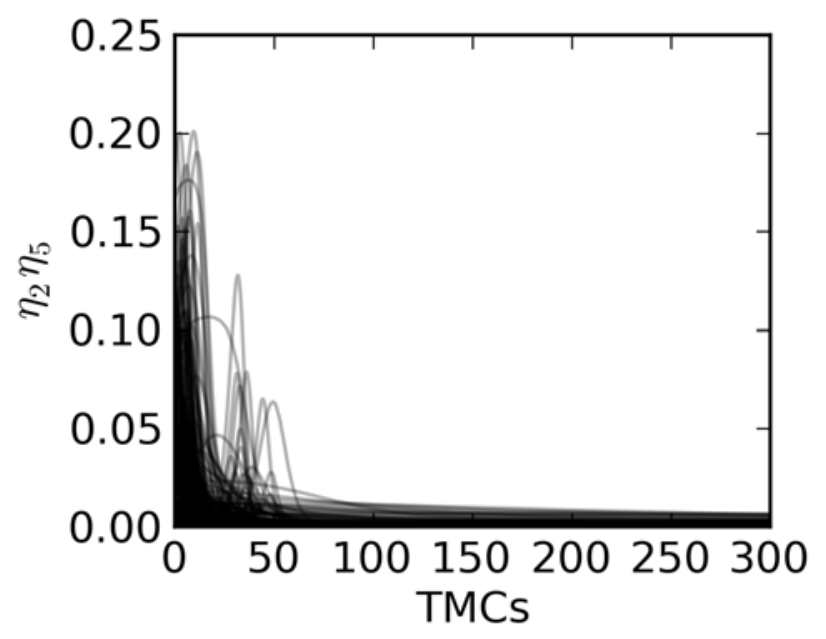

(a) $\eta_{1}+\eta_{2}+\eta_{5}$

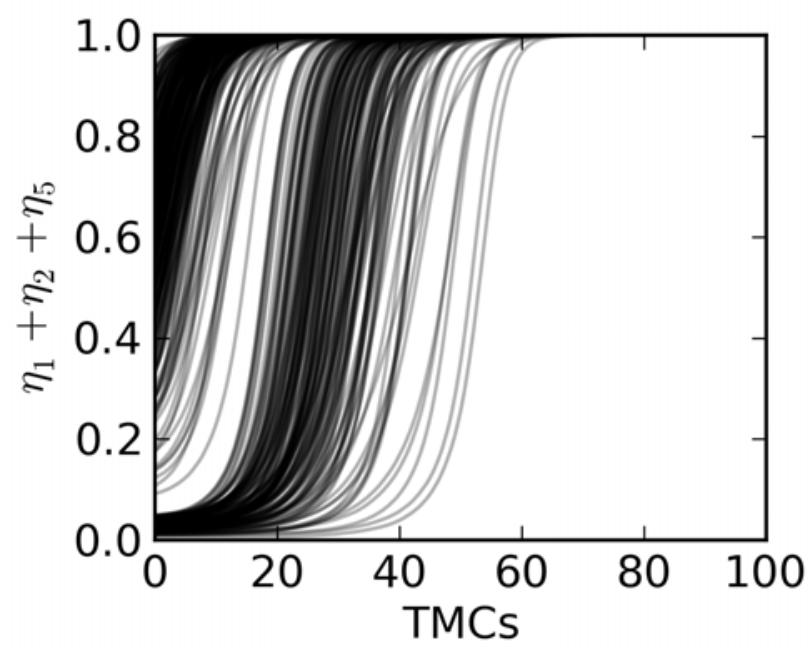

(b) $\eta_{2} \cdot \eta_{5}$

Figura 5.1.2. Séries temporais para a regra da figura 5.1 .1 onde os pesos são tomados como 0 ou 1 , mostrando os atratores da dinâmica. Note que na figura 5.1.2a o valor final é 1 (implicando que as opiniões 3 e 4 não sobrevivem). Na figura 5.1.2b o valor final é 0 , implicando que as opiniões $2 \mathrm{e}$ 5 não sobrevivem ao mesmo tempo.

a aresta $\overline{P_{2} P_{5}}$ ao vértice $P_{1}$. Logo, já levamos em conta os pontos provenientes dos subgrafos induzidos $\mathcal{R}_{\{1\}}$ e $\mathcal{R}_{\{2,5\}}$, ao considerar $\mathcal{R}_{\{1,2,5\}}$. As propriedades de estabilidade desses pontos se encontram na tabela 5.1.1. A projeção no espaço de fase (com os pesos da regra tomados como 0 ou 1), mostrando os atratores e as outras estruturas descritas na tabela se encontra na figura 5.1.3. 


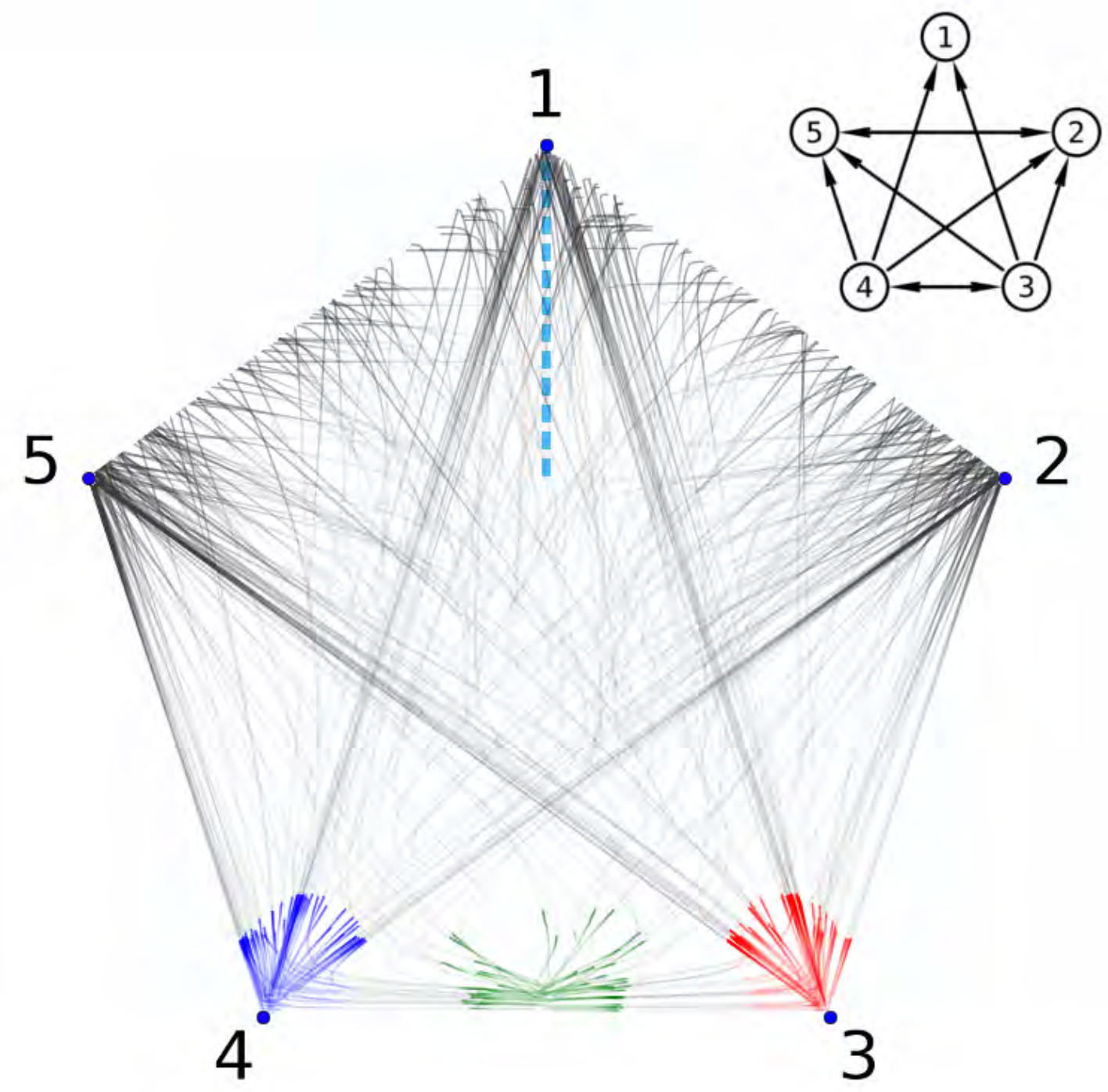

Figura 5.1.3. Projeção, no espaço de fase, do modelo com a regra da figura 5.1.1 no caso em que os pesos são 0 ou 1 mostrando as estruturas descritas na tabela 5.1.1 e os atratores. No canto superior direito temos um reoordenamento dos vértices da regra, explicitando os conjuntos independentes maximais. A linha tracejada em azul ciano mostra os pontos de sela $(1 \times 2,5$; na tabela 5.1.1), as trajetórias azuis são as que passam perto do ponto (4), as vermelhas passam perto de (3) e as verdes perto de $(3,4)$. Para todas essas trajetórias e as que estão em tons de cinza, cores mais claras indicam o início das trajetórias e as cores mais escuras indicam os finais delas. Podemos ver as trajetórias indo para os atratores $\mathcal{M}_{\{1,2\}}$ e $\mathcal{M}_{\{1,5\}}$, com algumas delas sendo primeiro atraídas pelas selas em $(1 \times 2,5)$ e depois repelidas. Também é possível ver as direções estáveis previstas para os pontos (3) e (4) e o fato que $(3,4)$ é um nó instável. 


\subsection{Opiniões inertes sobreviventes}

Agora vamos considerar 2 exemplos em que temos opiniões que sobrevivem em atratores, mesmo sem serem capazes de convencer nenhuma das outras (essas opiniões serão chamadas de inertes). Considere as regras nas figuras 5.2.1. Em $\mathcal{R}_{1}, \mathcal{M}_{\{1,3\}}$ é um atrator, apesar da opinião 1 ser inerte (não convence nenhuma das outras 3). Em $\mathcal{R}_{2}, \mathcal{M}_{\{4,5\}}, \mathcal{M}_{\{1,2\}}$ e $\mathcal{M}_{\{2,3\}}$ são os atratores, apesar da opinião 2, que aparece em 2 deles, ser inerte.

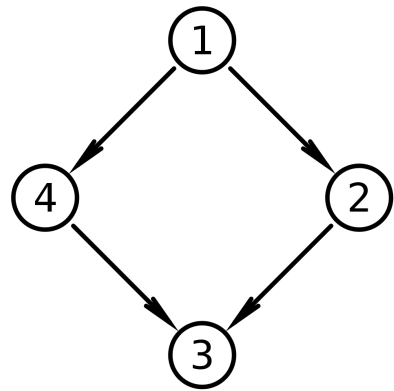

(a) $\mathcal{R}_{1}$

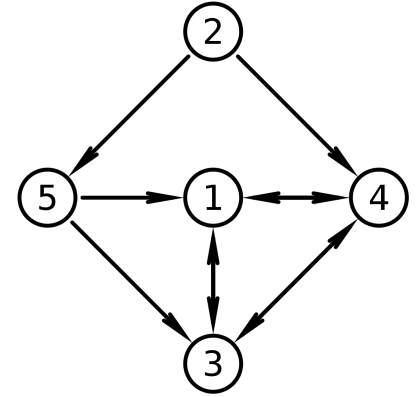

(b) $\mathcal{R}_{2}$

Figura 5.2.1. O esqueleto de 2 regras de confiança, $\mathcal{R}_{1}$ e $\mathcal{R}_{2}$, tais que opiniões inertes conseguem sobreviver em atratores estacionários.

Para checar que esse efeito esta presente nas simulações vamos olhar as séries temporais dos modelos com cada uma dessas regras (novamente, tomando os pesos como sendo 0 ou 1). Essas séries estão nas figuras 5.2.2a a 5.2.2d.

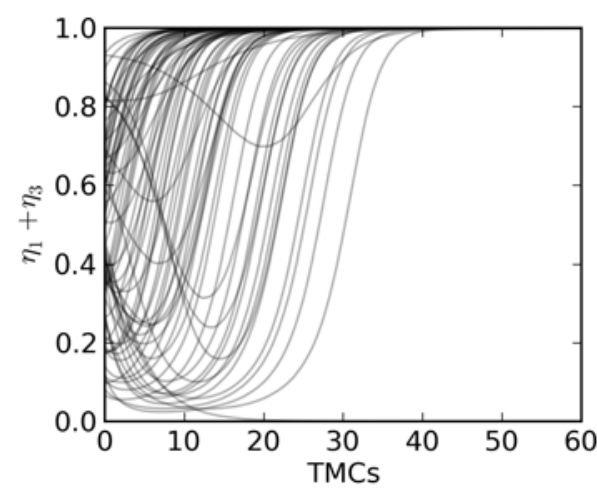

(a) $\left(\mathcal{R}_{1}\right) \eta_{1}+\eta_{3}$

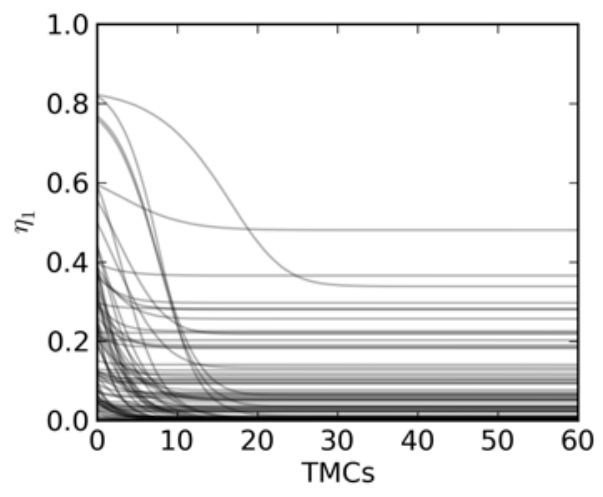

(b) $\left(\mathcal{R}_{1}\right) \eta_{1}$

Figura 5.2.2. Séries temporais para as regras $\mathcal{R}_{1}$ e $\mathcal{R}_{2}$. (continua) 


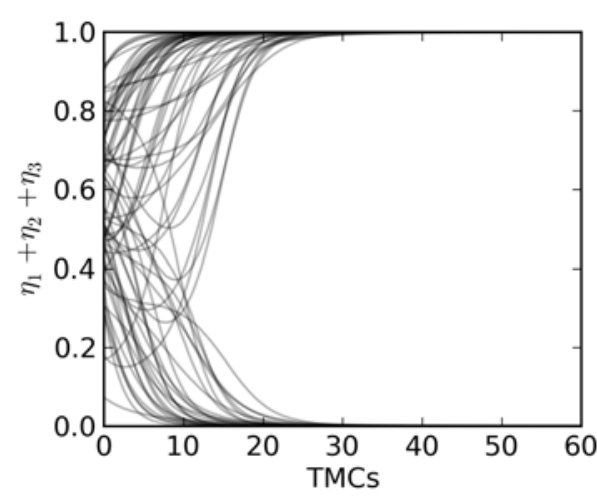

(c) $\left(\mathcal{R}_{2}\right) \eta_{1}+\eta_{2}+\eta_{3}$

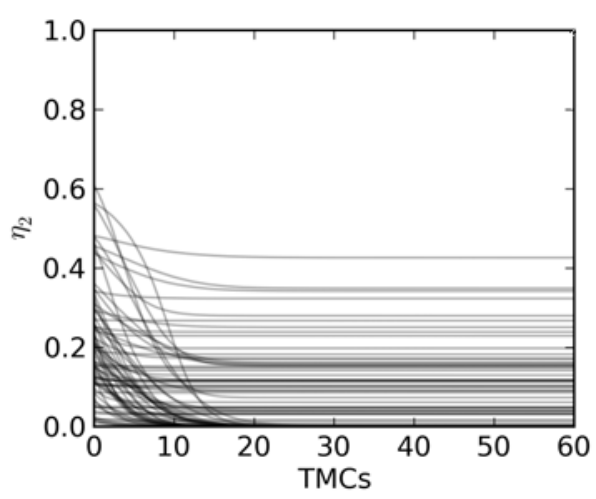

(d) $\left(\mathcal{R}_{2}\right) \eta_{2}$

Figura 5.2.2. Séries temporais para as regras $\mathcal{R}_{1}$ e $\mathcal{R}_{2}$ da figura 5.2 .1 com pesos 0 ou 1 . Os gráficos das figuras 5.2.2a e 5.2.2c mostram séries temporais que examinam o atrator como um todo (o valor final é 0 ou 1, dependendo do atrator alcançado), enquanto que os das figuras 5.2.2b e 5.2.2d focam as opiniões inertes sobreviventes (que sempre decaem mas podem atingir um valor estacionário não-nulo, indicando a sobrevivência).

\subsection{Regras sem atratores estacionários}

Considere uma regra em que todas as opiniões interagem (ou seja, para todo par de opiniões distintas, $\sigma$ e $\sigma^{\prime}$, temos que ou $p_{\sigma \rightarrow \sigma^{\prime}} \neq 0$ ou $p_{\sigma^{\prime} \rightarrow \sigma} \neq 0$ ), mas tal que toda opinião possui pelo menos uma opinião que ela não consiga convencer. Os conjuntos independentes dessa regra são todos unitários, mas nós acabamos de impor que, para todo $\sigma$, existe $\sigma^{\prime} \neq$ $\sigma$ tal que $\sigma^{\prime} \notin\{\sigma\}_{-}$, de forma que não existem soluções para $\Delta_{-}=\bar{\Delta}$ nessa regra $\mathrm{e}$ logo não temos atratores estacionários (é possível também construir outras regras com essa propriedade que não se enquadram nesse cenário). Um exemplo com 4 opiniões é dado na figura 5.3.1.

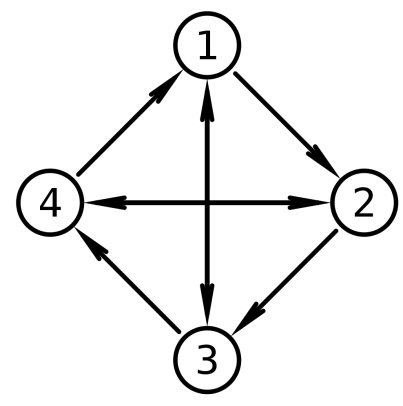

Figura 5.3.1. Uma regra sem atratores 
No apêndice A.1 (teorema A.1.2) nós provamos que, se um grafo não possui soluções para $\Delta_{-}=\bar{\Delta}$, então ele possui pelo menos um ciclo direcionado que não usa nenhuma aresta duplamente conectada. No espaço de fase, esses ciclos se traduzem em ciclos heteroclínicos. Esses ciclos vão ser sempre curvas poligonais conectando os vértices do simplexo correspondentes aos nós do ciclo no grafo. Além disso, se o ciclo passa pelos nós do conjunto $\Delta$, então $\Delta$ induz um grafo fortemente conexo na regra de confiança (e o grafo induzido possui somente uma componente), de forma que existe um ponto fixo em que todas as opiniões participantes do ciclo coexistem e que deve ser um nó instável (no exemplo da figura 5.3.1, o ciclo seria $1 \rightarrow 2 \rightarrow 3 \rightarrow 4 \rightarrow 1$, correspondendo ao ciclo heterocínico $\overline{P_{1} P_{2} P_{3} P_{4} P_{1}}$ e a um nó instável em que todas as opiniões coexistem). Olhando as trajetórias restritas à variedade $\mathcal{M}_{\Delta}$, isso significa que as trajetórias são repelidas do centro da variedade pelo nó e a medida que o tempo passa elas vão se aproximando cada vez mais do ciclo, que está completamente contido na borda de $\mathcal{M}_{\Delta}$. O resultado típico dessas regras é que, a medida que o tempo passa, as trajetórias se aproximam cada vez mais de um dos ciclos, o que causa oscilações com período que diverge no tempo. Durante as simulações, eventualmente uma flutuação aleatória faz com que uma das opiniões do ciclo seja extinta, levando o sistema a um estado estacionário.

\subsection{Transientes Longos e Estados Estacionários}

Em várias simulações observamos que ocorrem situações em que trajetórias ficam "presas" por longos períodos em estados que não são atratores. Em alguns desses casos, as simulações de fato congelaram, atingindo um estado estacionário em que não existem conexões ativas entre os agentes (ou seja, conexões entre um par de agentes que concordam entre si e um agente com uma opinião que possa ser convencida pela do par). Em outros casos existem conexões ativas, mas algumas das opiniões aparecem em quantidades desprezíveis e o conjunto $\Delta$ de opiniões que não são desprezíveis forma um conjunto independente, mas não uma solução de $\Delta_{-}=\bar{\Delta}$. No segundo caso, os pontos fixos em $\mathcal{M}_{\Delta}$ são selas, significando que (como mostramos no capítulo 4) $\widetilde{\Delta} \equiv\left(\bar{\Delta}-\Delta_{-}\right) \cap \Delta_{+} \neq \varnothing$ e, normalmente, uma (ou mais) das opiniões desprezíveis se reergue, causando um transiente longo.

Considerando as equações de campo médio, se $\sigma \in \widetilde{\Delta}$ (o que implica que $\sigma$ é desprezível), então ela evolui no tempo segundo ${ }^{1}$

$$
\eta_{\sigma}(t)=\frac{\eta_{\sigma \circ}}{1-\eta_{\sigma \circ} t \sum_{\sigma^{\prime} \in \Delta} \eta_{\sigma^{\prime} \mathrm{o}} p_{\sigma^{\prime} \rightarrow \sigma}}
$$

desde que as opiniões em $\bar{\Delta}$ continuem aparecendo em quantidades desprezíveis. Segue que o tempo gasto pelas trajetórias perto desses pontos de sela pode ser estimado, considerando o tempo que leva para que uma das opiniões em $\widetilde{\Delta}$ duplique a proporção em que ela aparece

${ }^{1}$ Maiores explicações estão no capítulo 4. 


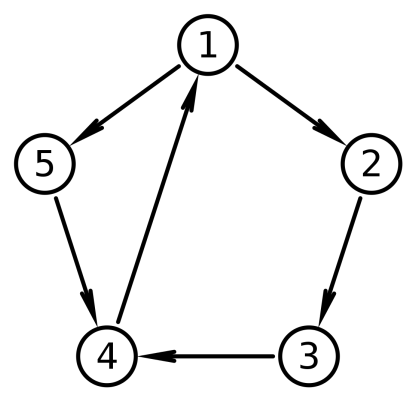

Figura 5.4.1. Uma regra com predisposição a exibir transientes longos e estados congelados.

na rede. Todas as outras opiniões em $\bar{\Delta}$ permanecem desprezíveis por tempos muito mais longos, como já discutido no capítulo 4. Resolvendo 5.4.1 temos

$$
\tau \simeq \min _{\sigma \in \widetilde{\Delta}}\left(\frac{1}{2 \eta_{\sigma \mathrm{o}} \sum_{\sigma^{\prime} \in \Delta} \eta_{\sigma^{\prime} \mathrm{o}} p_{\sigma^{\prime} \rightarrow \sigma}}\right)
$$

Podemos verificar essa relação para a integração das equações de campo médio e comparar esses resultados com as simulações. Nós iremos usar a regra da figura 5.4.1, com $\Delta=\{3,5\}$.

Para a nossa escolha de regra e de conjunto de opiniões, podemos simplificar 5.4.1 com

$$
\frac{1}{\eta_{4 \mathrm{o}}}-\frac{1}{\eta_{4}} \simeq t
$$

Os gráficos para o campo médio e para as simulações podem ser encontrados nas figuras 5.4.2a e 5.4.2b, que mostram que, se uma simulação não congela em um estado estacionário, então ela apresenta um período transiente que é muito mais rápido do que o previsto pelas equações de campo médio. 


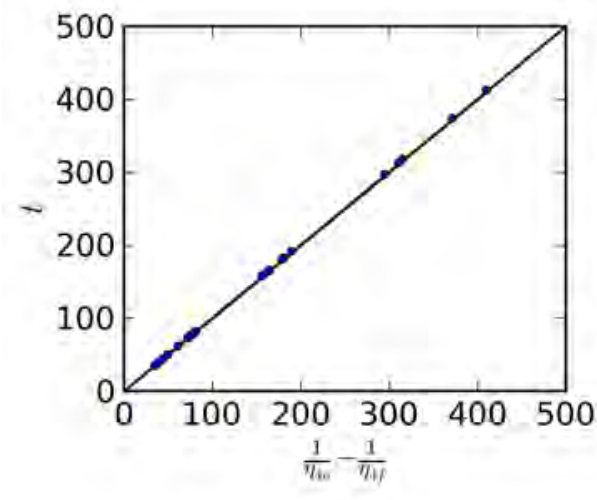

(a) Campo Médio

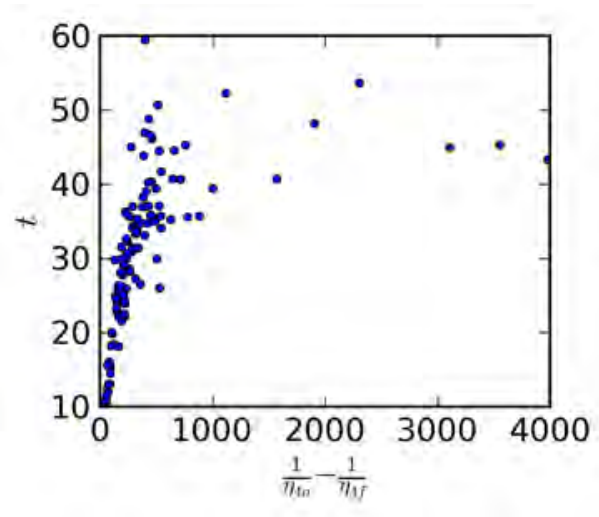

(b) Simulações em redes Barabási-Albert

Figura 5.4.2. Tempos de retorno para $\Delta=\{3,5\}$ (mais precisamente o tempo que a trajetória levou desde cruzar a superfície $\eta_{3}+\eta_{5}>1-\varepsilon$, até cruzar a superfície $\eta_{4}>2 \varepsilon$, com $\varepsilon=$ 0.025). Podemos ver que as simulações se comportam de forma bem diferente que o previsto pela aproximação de campo médio. Em particular, os tempos de retorno são menores do que os preditos pela equação 5.4.3 e a relação entre as 2 variáveis não é linear. Os pontos azuis correspondem aos dados obtidos através das simulações (na figura 5.4.2b) e das trajetórias integradas do campo médio (na figura 5.4.2a), enquanto que a linha em preto corresponde a previsão da equação 5.4.3 (que vale para a integração das equações de campo médio).

\subsection{Simulações em redes de Watts-Strogatz e competição cíclica}

Nessa seção nós iremos considerar a família de regras de confiança da figura 5.5.1 que pode ser interpretada como 2 partidos $(\{1,3\}$ e $\{2,4\})$ que possuem 2 facções cada uma (1, 3 e 2, 4 respectivamente). Além disso, temos uma competição cíclica entre as opiniões do tipo $A$ vence $B$ que vence $C$ que vence $D$ que vence $A$ e as 2 facções dentro de cada partido competem entre si de maneira simétrica.

Essa família possui 3 parâmetros $p, q, r$ e as suas regras serão denotadas por $\mathcal{R}(p, q, r)$. Estamos interessados no caso $p, q \neq 0$ (note que $r=0$ e $r \neq 0$ dá atratores diferentes no campo médio), de forma que os resultados de campo médio nos dizem que apenas um dos partidos sobrevive no estado estacionário.

Para as simulações nós usamos redes baseadas no modelo WS (ver seção 1.2.1), partindo de uma rede quadrada com condições periódicas de contorno e depois reconectando $s . E$ arestas, escolhidas aleatoriamente ( $s$ é um parâmetro de reconexão e $E$ é o número de arestas total da rede quadrada). Nas simulações, iremos sempre usar uma rede quadrada com lado $L=316$, como ponto de partida. Assim como nas outras simulações, exploramos todo o espaço de fase. Nesse caso vimos que, enquanto algumas simulações vão para estados esta- 


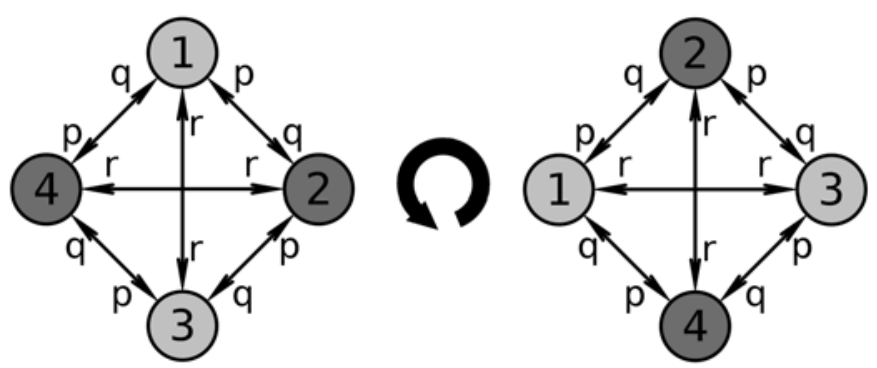

Figura 5.5.1. A regra de 2 partidos, $\mathcal{R}(p, q, r)$. Note que o grafo associado tem simetria de rotação proveniente da competição cíclica. Também segue que os 2 partidos (em cinza claro e escuro) são indistinguíveis, assim como as 4 opiniões. Estamos interessados principalmente nos casos com $p, q, r \neq 0$, cujos atratores de campo médio são os estados de consenso e $r=0$, mas com $p, q \neq 0$, em que os atratores são coexistências de facções dentro do mesmo partido (não há competição nesse caso, apenas falta de cooperação).

cionários inativos (os estados de consenso, que são estados absorventes e correspondem aos atratores do campo médio), um regime com oscilações estacionárias também é possível. Na figura 5.5.2 temos a série temporal da proporção de agentes que apóia um dos partidos, para diferentes simulações, usando uma regra em que esse comportamento pode ser observado para diferentes parâmetros de reconexão.

Podemos olhar a dependência desse comportamento com as propriedades da rede,mantendo a regra de confiança fixa e variando a reconexão (vamos usar $p=1, q=0.1$ e $r=0$ novamente). A medida que o parâmetro de reconexão aumenta, a distância média diminui e partes da rede que estavam originalmente distantes se aproximam. Definindo a amplitude das oscilações como sendo a média do desvio padrão, tomada sobre as simulações que exibem oscilações (usando uma janela móvel e após o transiente), vemos que a amplitude aumenta a medida que as reconexões aumentam, porém menos simulações passam a atingir o regime oscilatório, até que ele eventualmente deixa de existir. Na figura 5.5.3 temos a porcentagem de simulações que atingem esse regime, junto com a amplitude, em função da distância média.

A figura 5.5.2a mostra o comportamento típico das oscilações no caso com distância média alta $(\ell \simeq 42.6)$ e a figura 5.5.2b mostra um caso próximo da transição para o regime não-oscilatório $(\ell \simeq 20.3)$. Esses resultados podem ser melhor entendidos olhando o que está acontecendo no espaço de fase. As projeções do espaço de fase para valores diferentes de $s$ e para a rede BA estão na figura 5.5.4.

As oscilações nas figuras 5.5.2a e 5.5.2b vem de um atrator lembrando um ciclo limite, cujo tamanho aumenta a medida que as reconexões aumentam (figuras 5.5.4a, 5.5.4b e 5.5.4c), mas cujas bacias de atração diminuem (como visto na figura 5.5.3). Essas 2 condições parecem indicar que as bacias também ficam mais finas. 


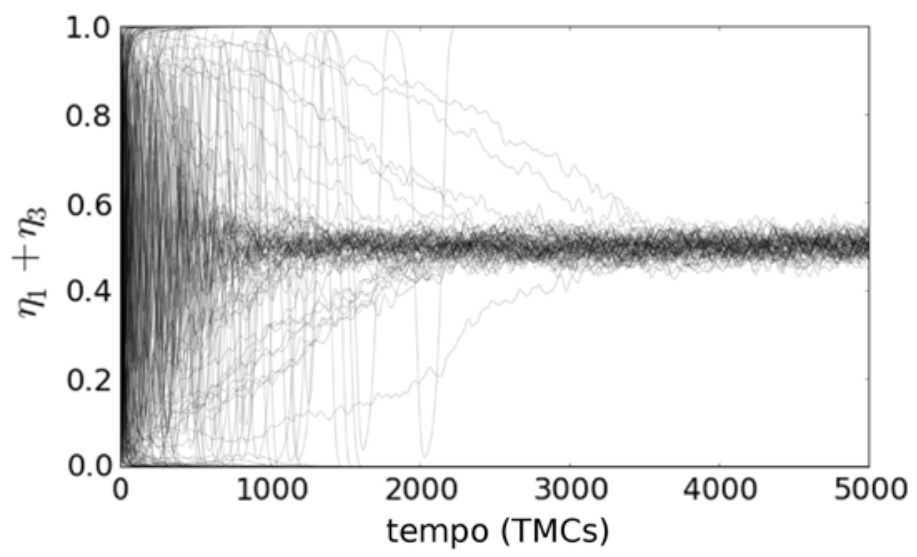

(a) $s=10^{-3}, \ell \simeq 42.6$

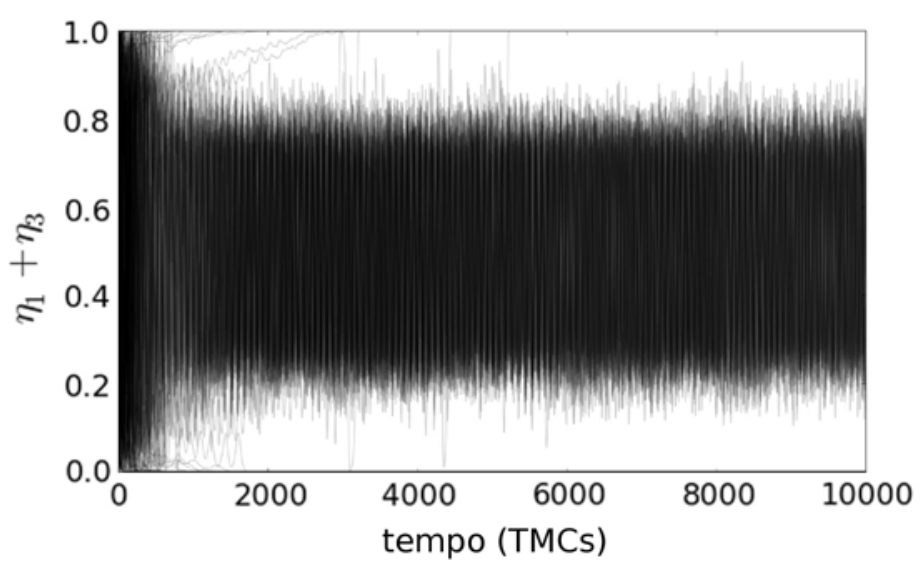

(b) $s=10^{-2}, \ell \simeq 20.3$

Figura 5.5.2. Séries temporais para $\eta_{1}+\eta_{3}$ no caso $p=1, q=0.1$ e $r=0$. Os parâmetros de reconexão e a distância média nas 2 redes são respectivamente $s=10^{-3}$ e $\ell=42.6$ e $s=10^{-2} \mathrm{e}$ $\ell=20.3$. 


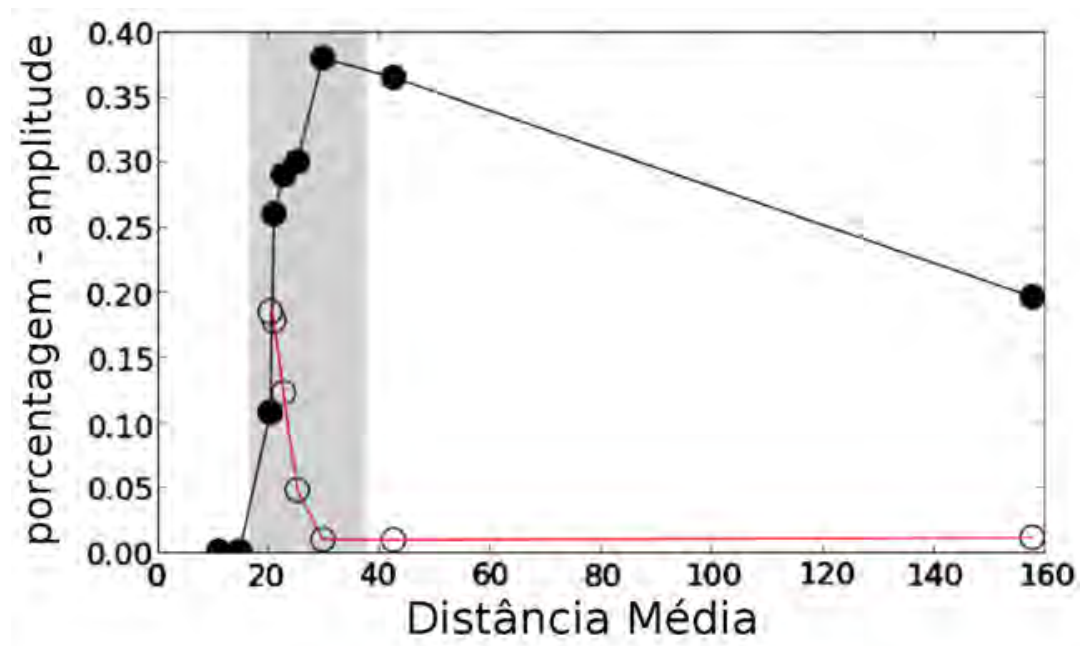

Figura 5.5.3. Amplitude das oscilações (o, curva vermelha) e porcentagem de simulações que atingem o regime oscilatório (•, curva preta). Note que não houveram simulações oscilando para $\ell \simeq 14.8 \mathrm{w} \ell \simeq 11.20$, correspondendo a parâmetros de reconexão $s=0.03$ e $s=0.1$ respectivamente. A região cinza é onde a transição entre existência e ausência de oscilações foi observada. A regra de confiança usada foi $p=1, q=0.1, r=0$.

Agora nós iremos fixar $s$ e explorar diferentes regras. Note que a única diferença entre as regras $\mathcal{R}(p, q, r)$ e $\mathcal{R}(\lambda p, \lambda q, \lambda r)$ (com $\lambda>0$ ) é a escala de tempo em que as coisas acontecem e que $\mathcal{R}(p, q, r)$ e $\mathcal{R}(q, p, r)$ são grafos isomorfos (um pode ser levado no outro mudando os "nomes" das opiniões), de forma que as propriedades da dinâmica (que não tenham a ver com escalas temporais) de todas essas regras devem ser iguais. Logo, podemos estudar o comportamento da família na figura 5.5.1 só com 2 parâmetros. Vamos focar no caso $r \neq 0$ e usar como parâmetros $p / r$ e $q / r$.

$\mathrm{Na}$ figura 5.5.5 temos a porcentagem de simulações que atingem o regime oscilatório para alguns valores de $\frac{p}{r}$ e $\frac{q}{r}$ (o parâmetro de reconexão é fixo em $s=3.10^{-3}$ ). Nessa figura podemos ver que as oscilações desaparecem quando $p \simeq q$, significando que algum grau de assimetria é necessário para o regime aparecer (e consequentemente a competição entre as opiniões é cíclica). 


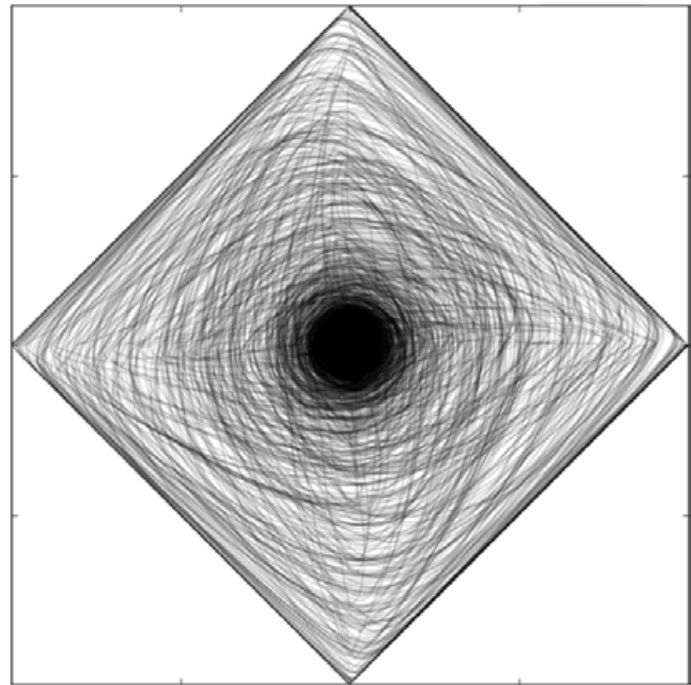

(a) $s=10^{-3}, \ell=42.6$

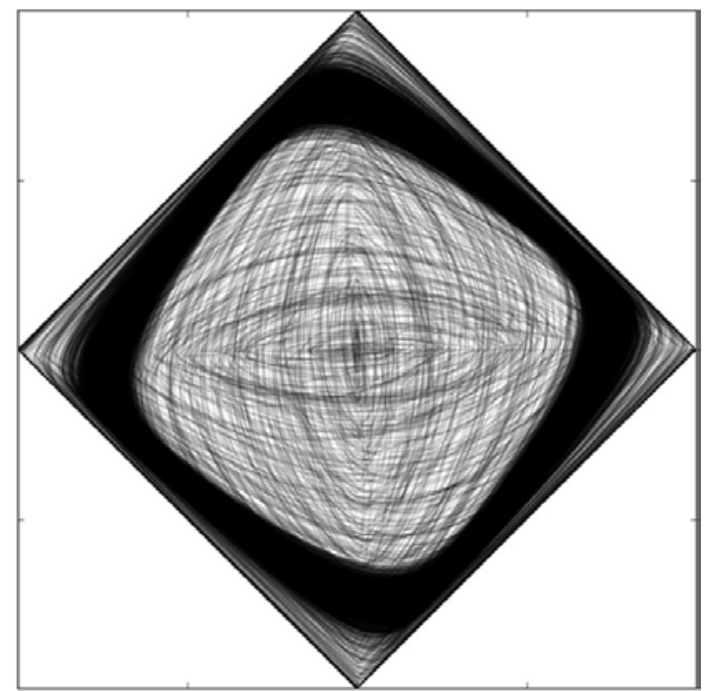

(c) $s=10^{-2}, \ell=20.3$

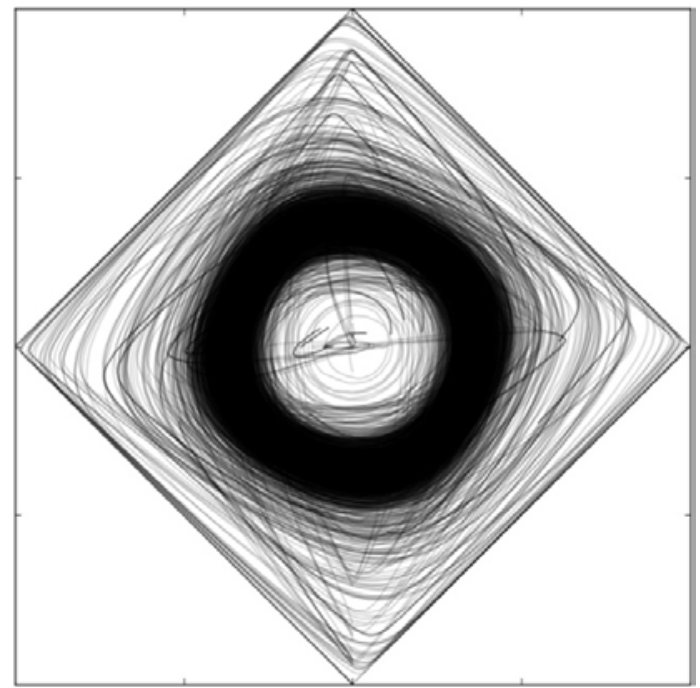

(b) $s=3.10^{-3}, \ell=29.8$

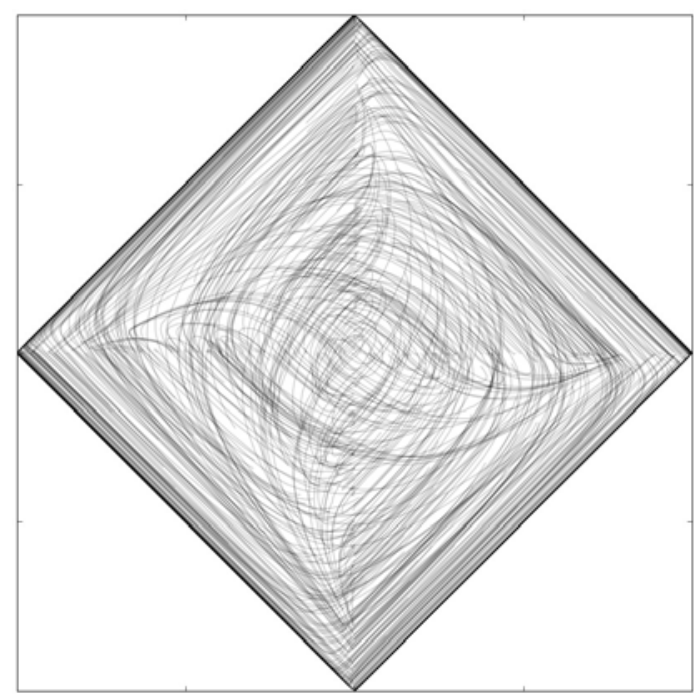

(d) Barabási-Albert, $\ell=4.3$

Figura 5.5.4. Projeção das trajetórias no espaço de fase para diferentes redes, usando a regra de confiança $p=1, q=0.1, r=0$. As redes nas figuras 5.5.4a a 5.5.4c são redes WS com diferentes parâmetros de reconexão e a figura 5.5.4d é a projeção para a rede BA com conectividade mínima 5 e tamanho $10^{5}$. Em todos os casos, as projeções dos 2 atratores do campo médio são as 2 diagonais do quadrado. (ou seja, estamos de fato fazendo o gráfico de $\eta_{1}-\eta_{3}$ por $\eta_{2}-\eta_{4}$ ) 


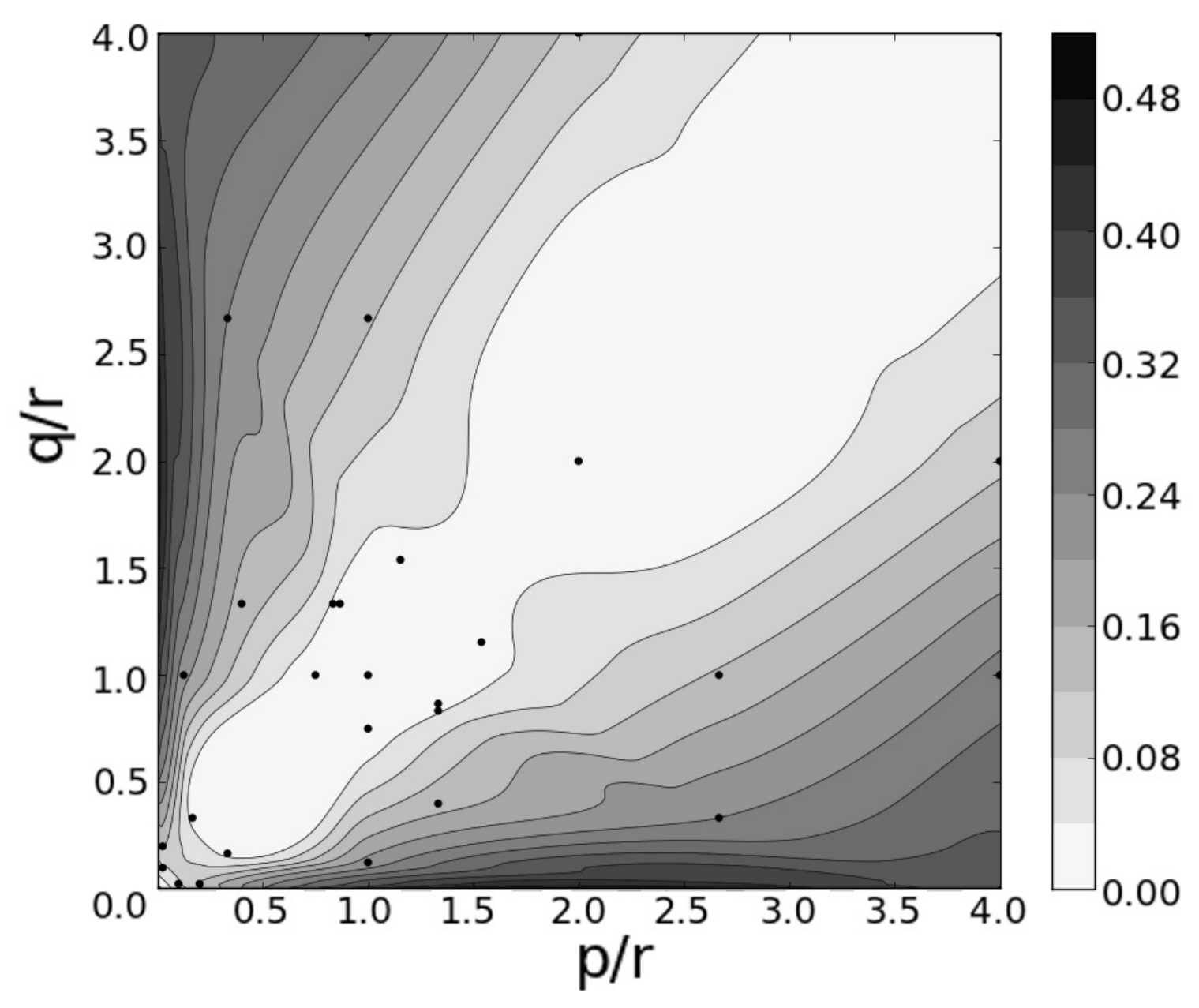

Figura 5.5.5. Porcentagem das simulações que terminaram no regime oscilatório em função de $\frac{p}{r}$ e $\frac{q}{r}$. A rede é fixa, com parâmetro de reconexão igual a $s=3 \cdot 10^{-3}$ e distância média aproximadamente 29.8. Este gráfico foi obtido usando splines cúbicos. Os pontos pretos representam os dados que foram de fato obtidos (alguns pontos na borda do quadrado e fora dele também foram medidos e levados em conta no cálculo do spline). Chamamos a atenção para os artefatos que esse tipo de interpolação causa, especialmente as "franjas" nas curvas de nível. Para melhorar a qualidade da interpolação, nós exploramos a simetria entre $p$ e $q$ para aumentar o número de pontos disponíveis e simetrizamos o resultado final. 


\subsection{Comparações quantitativas para o caso de 3 opiniões}

Como mencionamos nos resultados do modelo Sznajd (seção 2.4), o caso com 3 opiniões e confiança limitada tem resultados diferentes, quando simulado em redes quadradas e nas chamadas redes com vizinhos aleatórios, se partirmos de uma condição inicial em que todas as opiniões são sorteadas aleatoriamente e com a mesma probabilidade ${ }^{[43]}$. Para entender o que está acontecendo, vamos usar o modelo na aproximação de campo médio (o que é matematicamente equivalente ao modelo simulado na rede de vizinhos aleatórios) e uma rede Barabási-Albert no lugar da rede quadrada (que também reproduz o comportamento da rede quadrada, porém com simulações mais rápidas e menos ruído). Na figura 5.6.1 temos o espaço de fase do modelo com confiança limitada e 3 opiniões, com as trajetórias do campo médio e as trajetórias das simulações na rede (para tempos curtos). Apesar do campo médio ter trajetórias extremamente similares às das simulações, se a condição inicial uniforme for usada, o campo médio termina em consenso em $50 \%$ das vezes e em coexistência nos outros $50 \%$, enquanto que o modelo na rede BA quase sempre atinge o consenso.

$\mathrm{Na}$ figura 5.6.2 temos um detalhe do espaço de fase em torno do ponto correspondente a condição inicial que estamos estudando. Podemos ver que ocorre um deslocamento da bacia de atração por efeitos de rede e que corresponde a cerca de $1 \%$ das opiniões (ou seja, mudando as probabilidades de sorteio para que a nova condição inicial $\vec{\eta}_{\mathrm{o}}$ da rede BA esteja na bacia de coexistência, podemos fazer a mudança de forma que $\left\|\vec{\eta}_{\mathrm{o}}-\vec{\eta}_{u}\right\| \simeq 1 / 100$, onde $\vec{\eta}_{u}$ é a condição inicial uniforme, que estamos de fato estudando), de forma que a condição inicial cai dentro da bacia de atração do estado de consenso para a rede BA, enquanto que ela fica exatamente na borda da bacia de atração para as equações de campo médio (o que explica o resultado de $50 \%$ de probabilidade para cada atrator).

Os espaços de fase para os outros esqueletos não-direcionados (sempre com pesos $0 \mathrm{ou}$ 1 , regras de uma componente e eliminando casos equivalentes), se encontram nas figuras $5.6 .3 \mathrm{a}$ a $5.6 .3 \mathrm{k}$ 


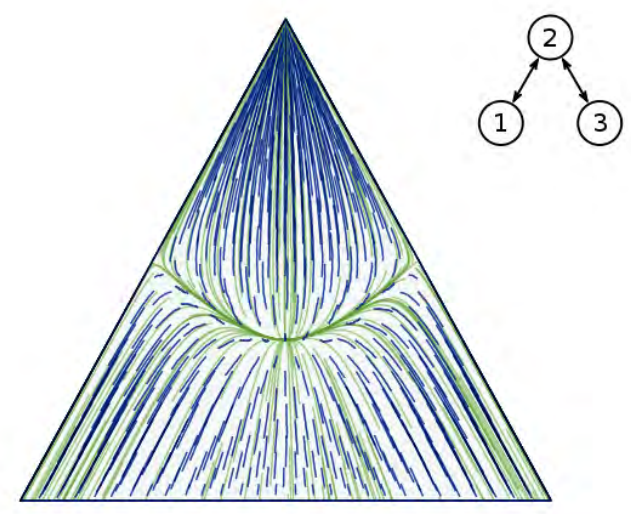

Figura 5.6.1. Espaço de fase para o modelo com 3 opiniões e confiança limitada. Podemos ver que as trajetórias do campo médio e as das simulações de tempos curtos são extremamente parecidas, apesar do comportamento diferente para condições iniciais sorteadas uniformemente. As trajetórias em verde correspondem ao campo médio e as trajetórias em azul são as simulações na rede BA.

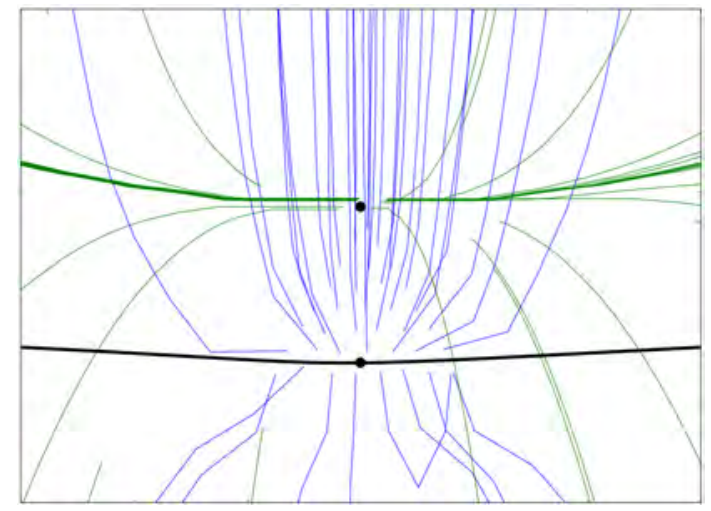

Figura 5.6.2. Detalhe do espaço de fase em torno da condição inicial uniforme. Novamente, as trajetórias em verde correspondem ao campo médio e as trajetórias em azul às simulações na rede BA. Os pontos marcados são os nós instáveis em que as 3 opiniões coexistem, para os 2 casos. Esse ponto fixo é no caso do campo médio exatamente o ponto que corresponde a condição inicial uniforme. Podemos ver claramente a fronteira entre as bacias de atração para o caso do campo médio e podemos estimar essa fronteira para o caso da rede BA (a curva em preto). Fica claro que as probabilidades de $50 \%$ encontradas no caso de vizinhos aleatórios são devidas inteiramente a flutuações no sorteio das condições iniciais, enquanto que o comportamento da rede BA para essa condição inicial segue do deslocamento sofrido pela fronteira das bacias de atração, que aumenta o tamanho da bacia de consenso. A distância entre os 2 pontos fixos é 1.5\% do comprimento de uma das arestas do espaço de fase (imerso de forma que todas tenham o mesmo comprimento). 


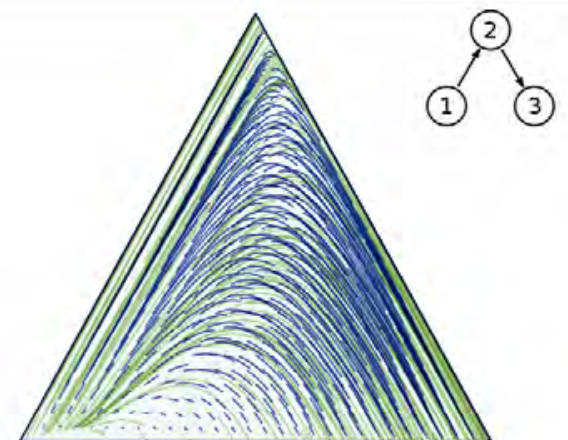

(a)

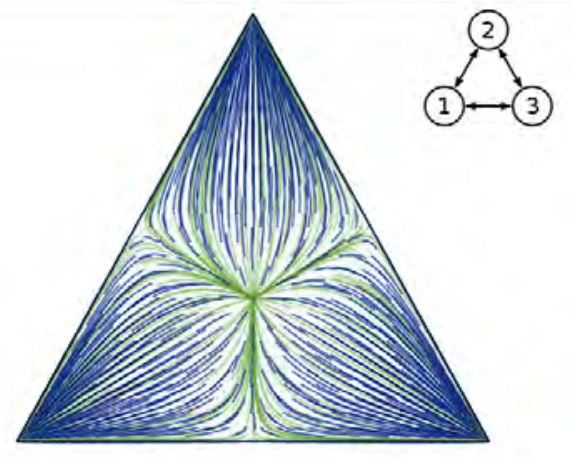

(b) O modelo usual

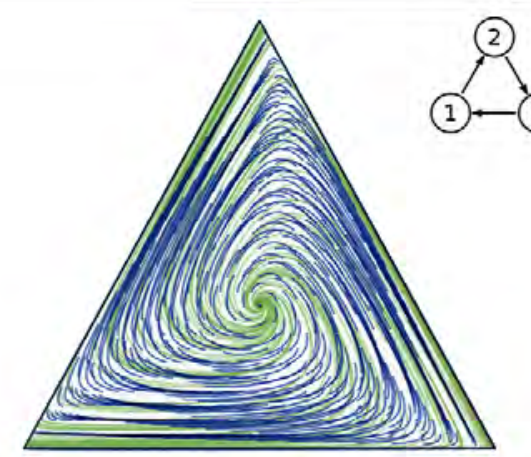

(c)

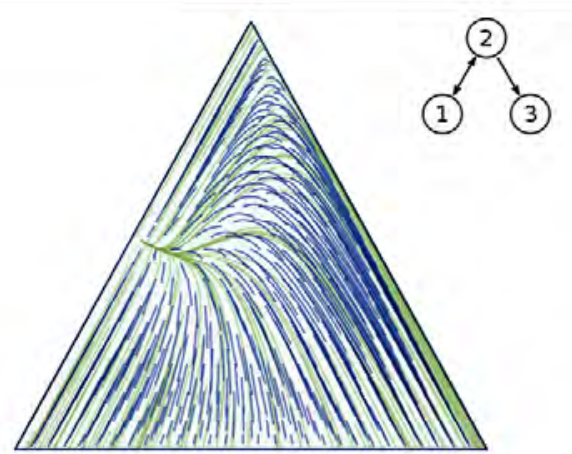

(d)

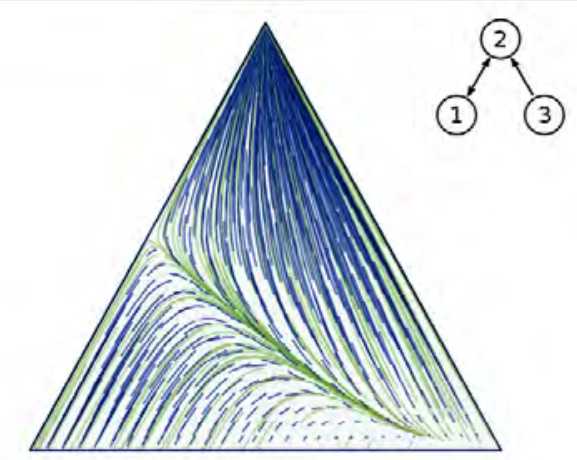

(e)

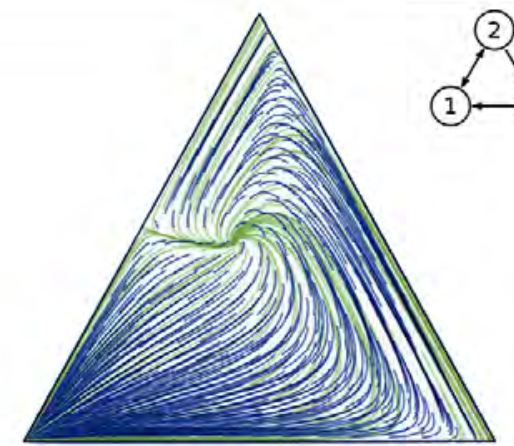

(g)

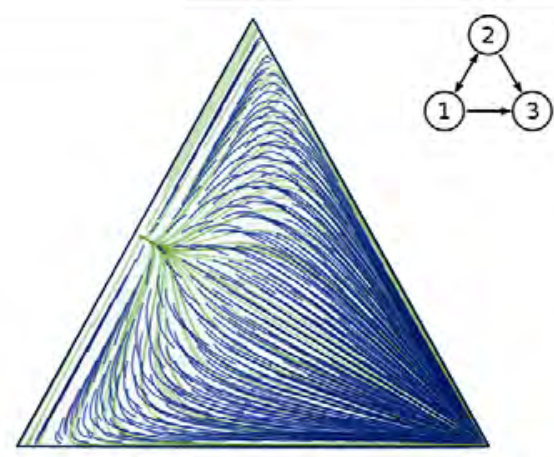

(f)

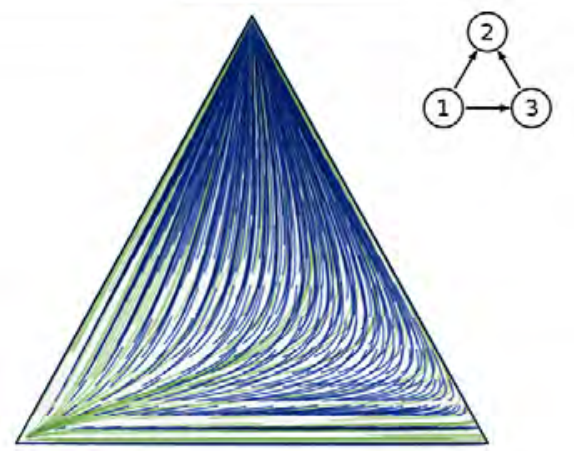

(h)

Figura 5.6.3. Espaços de fase para 3 opiniões (continua) 


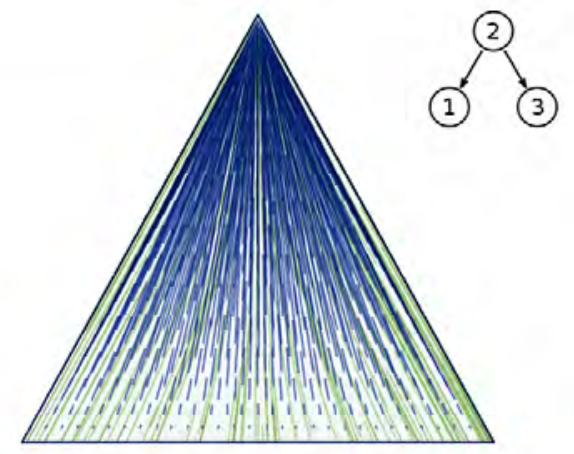

(i)

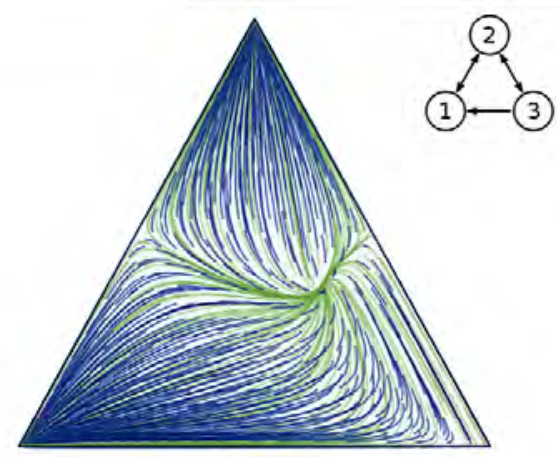

(j)

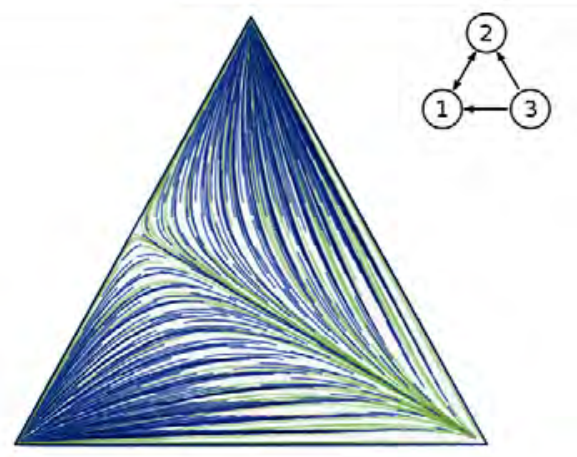

(k)

Figura 5.6.3. Os espaços de fase para os diferentes esqueletos direcionados (excluindo o caso com confiança limitada, que foi estudado separadamente, casos em que as equações podem ser integradas e esqueletos com mais de uma componente). Todos os pesos das regras de confiança são 0 ou 1 . 


\subsection{Modelo com regras de confiança e ruído}

Vamos agora apresentar um exemplo de como funcionam na prática os resultados da teoria de perturbação, desenvolvida na seção 4.4.2. Para tanto, vamos estudar a regra de confiança da figura 5.7.1. Os pontos fixos do modelo sem ruído são os pontos em que as opiniões 1 e 3 coexistem (que são atrativos) e o ponto em que temos consenso da opinião 2 (que é uma sela). Logo, os únicos pontos que sobrevivem à adição do ruído são tais que $\Delta=\{1,3\}$ e $\Omega=\{2\}$. As 2 componentes induzidas por $\Delta$ são $\{1\}$ e $\{3\}$, de forma que trivialmente $\zeta_{1}=\zeta_{3}=1$. Além disso, $\lambda_{2}=-p_{2 \rightarrow 3} \eta_{3}^{2}$. Escolhendo então os pesos da regra de confiança como 0 ou 1 , vem $\lambda_{2}=-\eta_{3}^{2}$. Se usarmos o ruído uniforme, então $G_{\sigma}=1$, se $\eta_{\sigma}=0$.

A equação de robustez 4.4 .34 para a componente $\{1\}$ fica

$$
\eta_{1}^{2}\left(\frac{p_{2 \rightarrow 1}}{\eta_{1}^{2} p_{2 \rightarrow 1}+\eta_{3}^{2} p_{2 \rightarrow 3}}\right)-3 \eta_{1}+1=0 .
$$

Mas como $p_{2 \rightarrow 1}=0$ (o arco $2 \rightarrow 1$ está ausente no esqueleto direcionado da regra), então o único ponto robusto do modelo sem ruído é

$$
\eta_{1}=\frac{1}{3}, \quad \eta_{2}=0, \quad \eta_{3}=\frac{2}{3} .
$$

A única previsão que podemos fazer sobre o ponto fixo após a adição do ruído são os valores de $\psi_{2}$ e $\psi_{1}+\psi_{3}$, onde $\vec{\psi}$ é a derivada do ponto fixo com respeito a intensidade de ruído $\theta$ (como vimos na seção 4.4.2, só podemos determinar $\vec{\psi}$ a menos de $k-1$ variáveis livres, onde $k$ é o número de componentes, induzidas por $\Delta$ ). Usando a equação 4.4.19, então

$$
\psi_{2}=\frac{-1}{\lambda_{2}}=\frac{1}{\eta_{3}^{2}}
$$

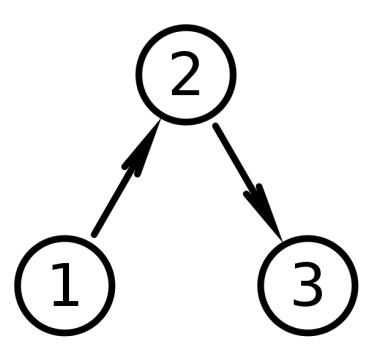

Figura 5.7.1. Regra que será usada no nosso exemplo do funcionamento da teoria de perturbação. 


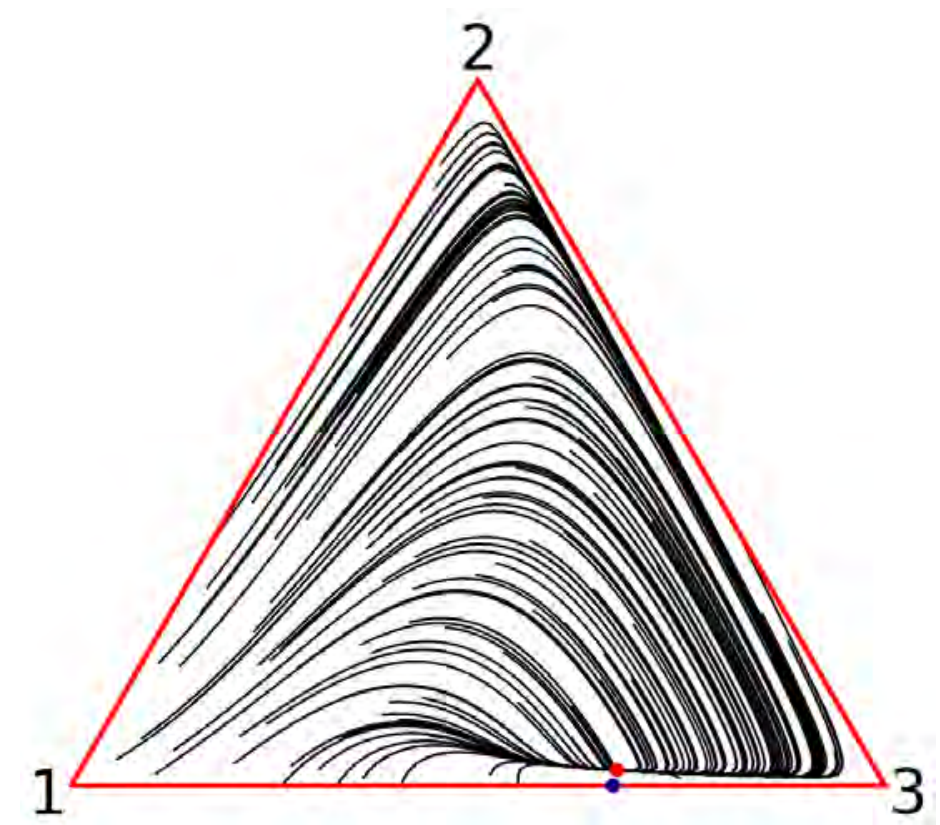

Figura 5.7.2. Espaço de fase do modelo no campo médio com a regra de confiança 5.7 .1 e ruído de intensidade $\theta=0.01$. O ponto azul é o ponto robusto do modelo sem ruído e o vermelho é o ponto fixo do modelo com ruído.

e substituindo 5.7.1 temos $\psi_{2}=9 / 4=2.25$. Usando agora a segunda equação em 4.4.18, segue que $\psi_{1}+\psi_{3}=-\psi_{2}=-9 / 4$. O espaço de fase do modelo simulado com essa regra de confiança e intensidade de ruído $\theta=0.01$ pode ser encontrado na figura 5.7.2. O valor de $\psi_{2}$ medido a partir dessas simulações foi $\psi_{2}^{(\text {sim })}=2.24846(1)$, que dá um erro relativo de $7.10^{-4}$. Os outros valores são $\psi_{1}^{(\text {sim })}=-0.55242(1)$ e $\psi_{3}^{(\text {sim })}=-1.69604(1)$.

Para estudar a variação dos autovalores do jacobiano avaliado no ponto fixo, podemos calcular a matriz $\mathfrak{G}$ pela equação 4.4 .58 , obtendo

$$
\mathfrak{G}=\left[\begin{array}{cc}
-2 & 1 \\
2 & -1
\end{array}\right]
$$

Um dos autovalores de $\mathfrak{G}$ é sempre 0 (isso foi deduzido na seção 4.4.2), por causa do artefato de imersão, de forma que o outro autovalor é o traço de $\mathfrak{G}$. Logo, quando $\theta$ é pequeno, um dos autovalores vai ser aproximadamente $\lambda_{2}=-4 / 9$, mais um termo de ordem $\mathcal{O}(\theta)$, enquanto que o outro será igual a $-3 \theta$.

\subsection{O modelo do votante}

Fizemos simulações para o modelo do votante em uma rede Barabási-Albert com tamanho $10^{5}$ e $m=5$, com uma regra de 3 opiniões, para verificar como as oscilações previstas na 
aproximação de campo médio se comportam. O comportamento do campo médio depende unicamente da matriz $T_{\sigma, \sigma^{\prime}}=p_{\sigma^{\prime} \rightarrow \sigma}-p_{\sigma \rightarrow \sigma^{\prime}}$. Usando a matriz

$$
T_{3}=\left[\begin{array}{ccc}
0 & 0.4151348 & -0.95238095 \\
-0.4151348 & 0 & 0.88923167 \\
0.95238095 & -0.88923167 & 0
\end{array}\right]
$$

que tem ponto fixo $(0.394,0.422,0.184)$ e tomando os parâmetros da regra de confiança como sendo

$$
p_{\sigma \rightarrow \sigma^{\prime}}=\max \left\{0, T_{\sigma^{\prime}, \sigma}\right\},
$$

obtemos os espaços de fase da figura 5.8.1. O comportamento oscilatório aparece na rede, porém as trajetórias vão se afastando do centro com o tempo e depois de um transiente o sistema se ordena. Podemos ver isso no detalhe do espaço de fase que se encontra na figura 5.8.2, assim como na série temporal de $Q=\vec{\eta}^{*} \cdot \vec{L}(\vec{\eta})$ (ver a seção 4.6 para maiores detalhes), que é uma constante no campo médio e que tende a $-\infty$ para estados ordenados (figura 5.8.3).

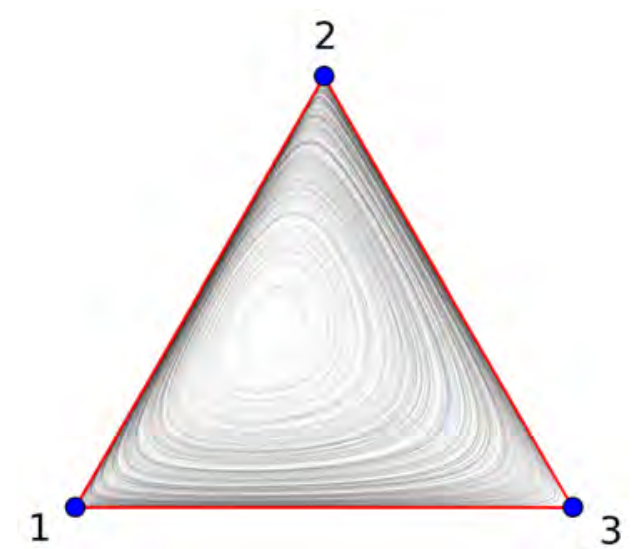

(a) Espaço de fase para o modelo do votante na rede Barabási-Albert

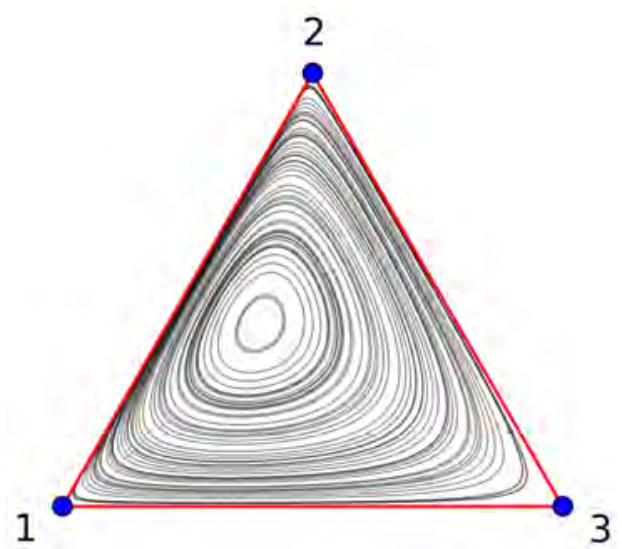

(b) Espaço de fase para o modelo do votante na aproximação de campo médio

Figura 5.8.1. Espaço de fase para o modelo do votante, em um caso com 3 opiniões em que temos um ponto fixo em que todas as opiniões coexistem.

A seguir olhamos um caso com 4 opiniões em que temos quasiperiodicidade. Para tanto, usamos o seguinte caso em que a matriz $T$ tem multiplicidade 2 para o autovalor 0 , mas todas as opiniões interagem entre si: 


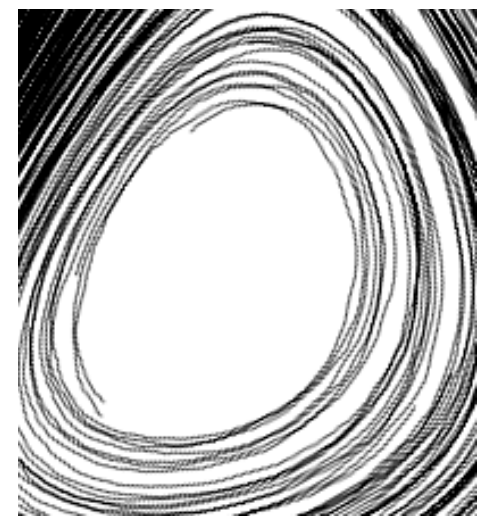

Figura 5.8.2. Detalhe do espaço de fase, mostrando que as trajetórias descrevem espirais se afastando da região onde se encontra o ponto fixo (estados desordenados) e vão para as bordas do espaço de fase (estados ordenados).

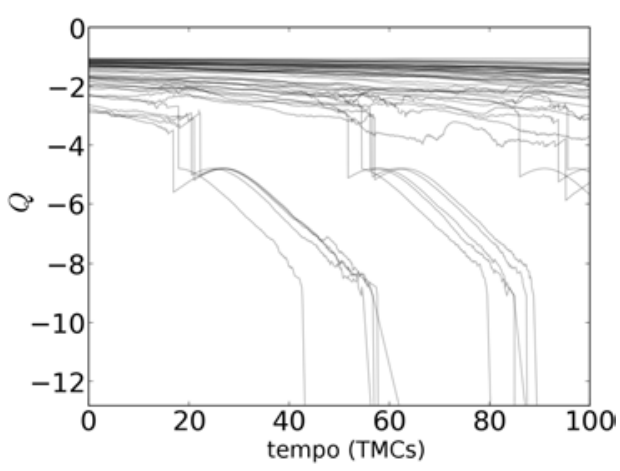

(a) Série temporal para tempos curtos

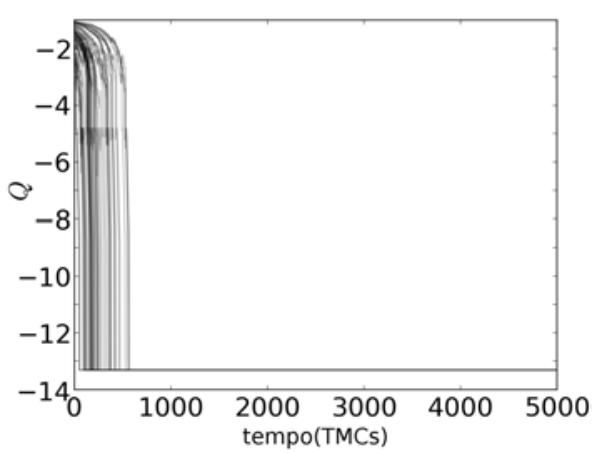

(b) Série temporal para tempos longos

Figura 5.8.3. Série temporal na rede da grandeza conservada do campo médio $(Q)$. Aqui ao invés de usarmos a série temporal de $\sum_{\sigma} \eta_{\sigma}^{*} \ln \left(\eta_{\sigma}\right)$, estamos usando $\sum_{\sigma} \eta_{\sigma}^{*} \ln \left(\eta_{\sigma}+\epsilon\right)$, com $\epsilon=10^{-10}$, de forma que podemos continuar acompanhando a evolução mesmo depois que uma das variáveis vá a 0 . Isso também permite extrair outras informações do gráfico. Por exemplo, a altura das quedas indica que as quedas bruscas vistas no gráfico para tempos curtos são causadas pela extinção da opinião 3, de forma que a opinião 2 sempre é a única sobrevivente nessas simulações, o que também pode ser deduzido pelo valor do patamar final, no gráfico para tempos longos. 


$$
T_{4}=\left[\begin{array}{cccc}
0 & -0.2584532 & 0.08044741 & 0.06948171 \\
0.2584532 & 0 & 0.44129866 & -0.77332313 \\
-0.08044741 & -0.44129866 & 0 & 0.35934563 \\
-0.06948171 & 0.77332313 & -0.35934563 & 0
\end{array}\right]
$$

e aproximamos os termos até a terceira casa decimal para obter

$$
T_{4}^{\prime}=\left[\begin{array}{cccc}
0 & -0.258 & 0.08 & 0.069 \\
0.258 & 0 & 0.441 & -0.773 \\
-0.08 & -0.441 & 0 & 0.359 \\
-0.069 & 0.773 & -0.359 & 0
\end{array}\right]
$$

As trajetórias do campo médio e da rede Barabási-Albert podem ser encontradas na figura 5.8.4. Iremos chamar as grandezas conservadas do campo médio para a matriz $T_{4}$ de $Q_{1}$ e $Q_{2}$ (usamos o autovetor $(0.359,0.144,0.24,0.257)$ para $Q_{1}$ e o autovetor $(0.803,-0.189$, $-0.562,-0.052)$ para $\left.Q_{2}\right)$. As séries temporais dessas grandezas, na aproximação de campo médio, estão nas figuras 5.8.5a e 5.8.5c. Nas figuras 5.8.4a a 5.8.4c pudemos perceber algumas semelhanças no formato das trajetórias, mas o modelo com a matriz $T_{4}$ simulado na rede Barabási se ordena muito mais rapidamente, apesar de algumas simulações terem permanecido até o final no estado oscilante, como evidenciado pela série temporal das grandezas conservadas do campo médio nas figuras $5.8 .5 \mathrm{~b}$ e 5.8.5d. Além disso, esses estados oscilantes não sobrevivem se usarmos ao invés da regra dada na equação 5.8.1, por exemplo, a regra de confiança

$$
p=\left[\begin{array}{cccc}
0 & 0.11450515 & 0.20662159 & 0.19406529 \\
0.41044739 & 0 & 0.61981487 & 0.11450515 \\
0.11450515 & 0.11450515 & 0 & 0.52597442 \\
0.11450515 & 1 & 0.11450515 & 0
\end{array}\right] .
$$

que também tem o comportamento de campo médio da matriz $T_{4}$. As séries temporais desse caso estão nas figuras 5.8 .5 e e 5.8.5f. 


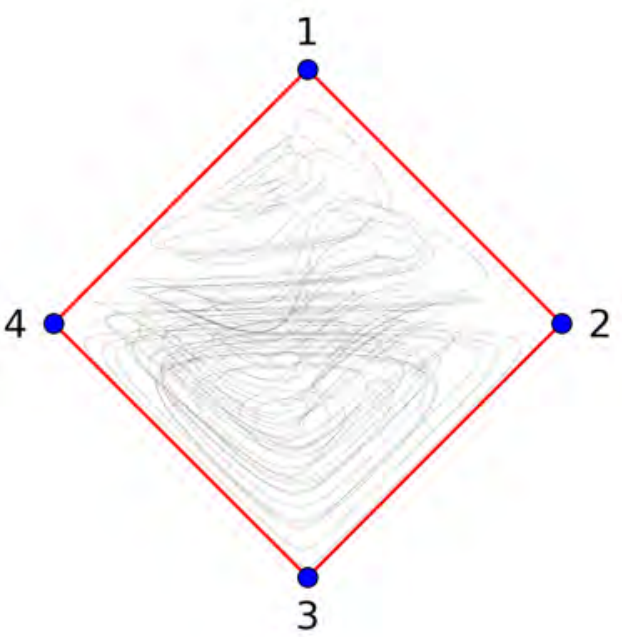

(a) $T_{4}$ (campo médio)

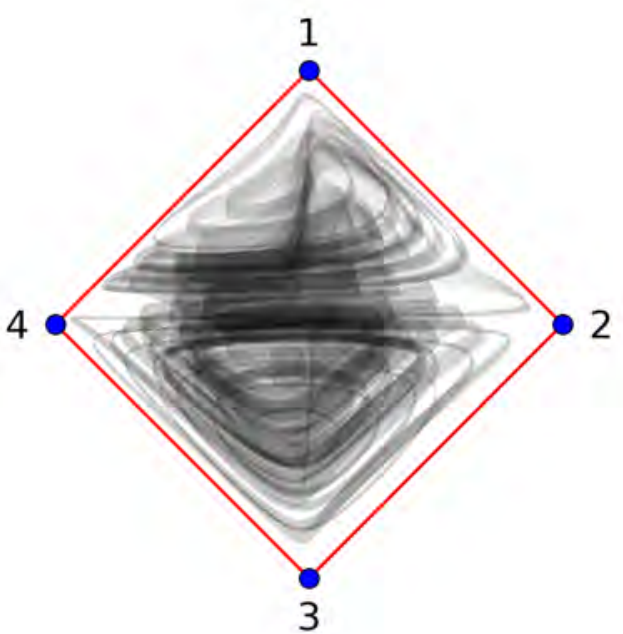

(b) $T_{4}^{\prime}$ (campo médio)

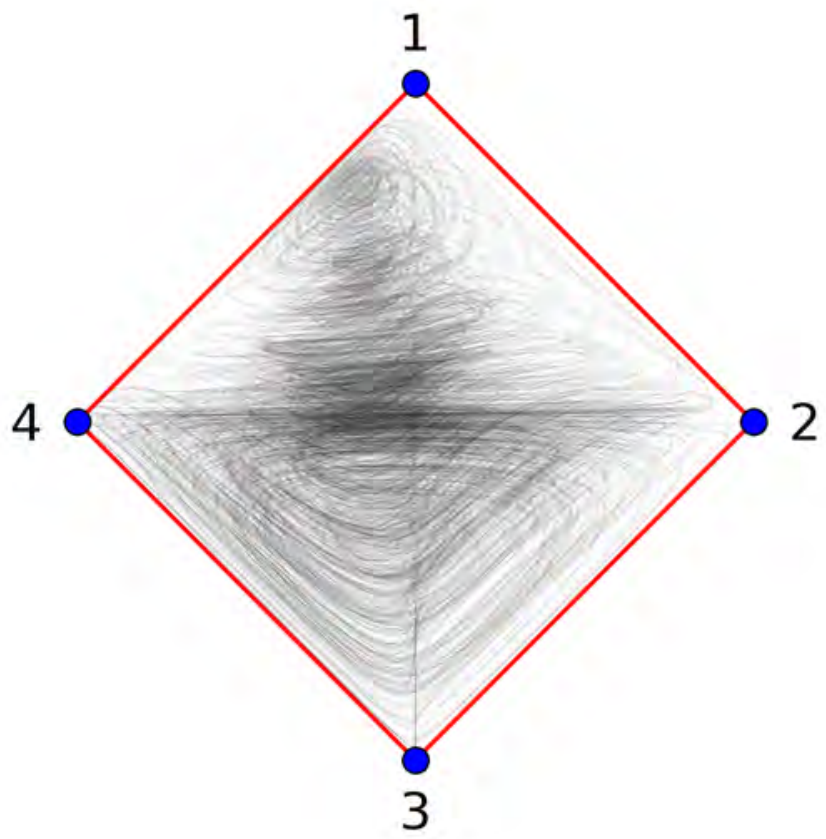

(c) $T_{4}$ (Barabási-Albert)

Figura 5.8.4. Espaço de fase para o modelo do votante na aproximação de campo médio, em um caso com 4 opiniões, usando as matrizes $T_{4}$ e $T_{4}^{\prime}$ e para o modelo na rede Barabási-Albert, usando $T_{4}$. 


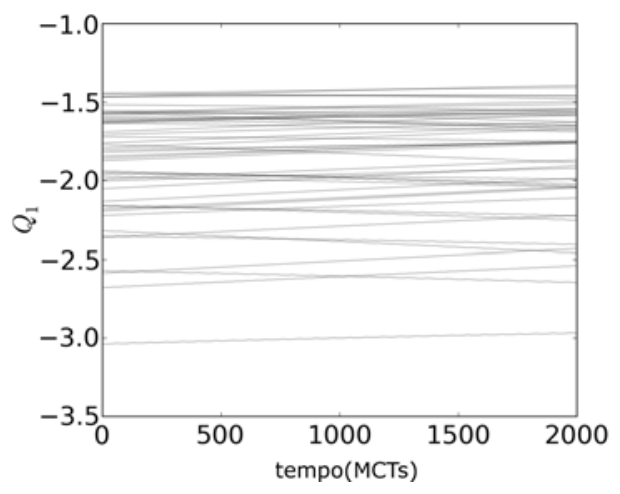

(a) $Q_{1}$ (campo médio usando $T_{4}^{\prime}$ )

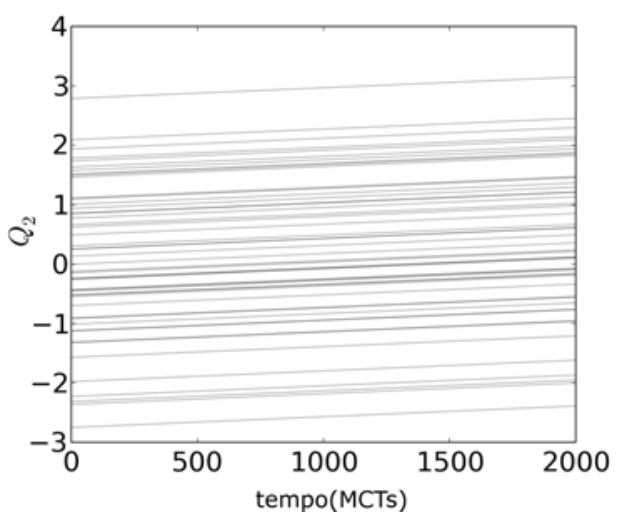

(c) $Q_{2}$ (campo médio usando $T_{4}^{\prime}$ )

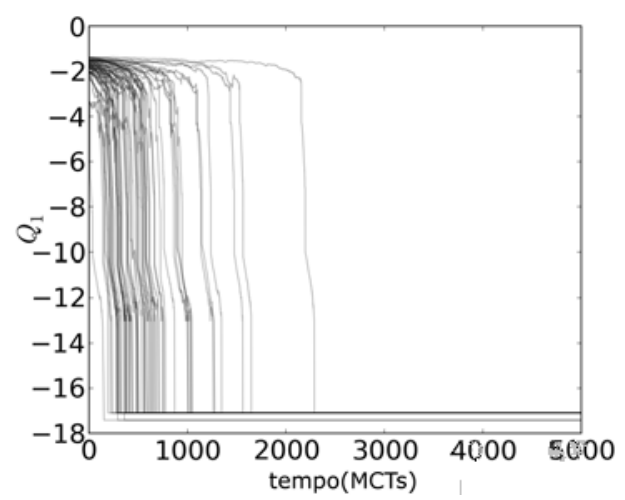

(e) $Q_{1}$ (Barabási-Albert usando a regra modificada)

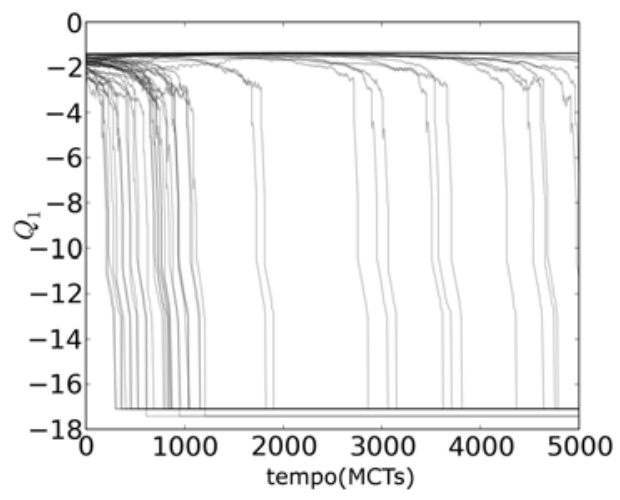

(b) $Q_{1}$ (Barabási-Albert usando $T_{4}$ )

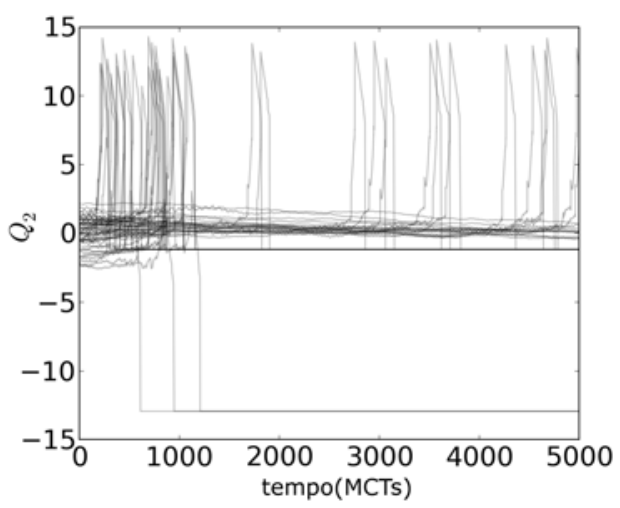

(d) $Q_{2}$ (Barabási-Albert usando $T_{4}$ )

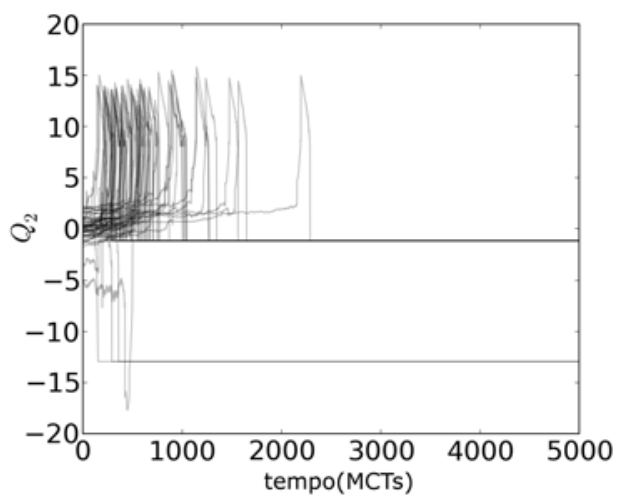

(f) $Q_{2}$ (Barabási-Albert usando a regra modificada)

Figura 5.8.5. Série temporal das grandezas conservadas do campo médio $\left(Q_{1}\right.$ e $\left.Q_{2}\right)$. Valem as mesmas observações a respeito da figura 5.8.3. Podemos ver algumas oscilações que se mantém após 5000 tempos de montecarlo e simulações que terminam em consenso da opinião $4\left(Q_{2}=\right.$ $-1.2)$ e em consenso da opinião $3\left(Q_{2}=-12.9\right)$. Nas séries para a regra dada em 5.8.2 as oscilações sobreviventes não estão presentes. 


\subsection{O modelo rock-paper-scissors de biodiversidade}

O modelo RPS (rock-paper-scissors) é um modelo de biodiversidade definido em uma rede quadrada com condições periódicas de contorno, em que os sítios podem ocupar 4 estados: pedra $(R)$, papel $(P)$, tesoura $(S)$ e vazio $(E)$. Os estados $R, P$ e $S$ representam as espécies ${ }^{[32]}$, porém mais adiante também vamos denotar estados genéricos (tanto as espécies $R, P, S$ quanto o estado vazio $E$ ) por $X$ ou $Y$. Revisitamos o modelo, introduzindo uma assimetria na interação entre as espécies (essa parte do trabalho foi feita em colaboração com o Institute for Complex Systems and Mathematical Biology da Universidade de Aberdeen). As regras do modelo (já com a nossa introdução de assimetria) são controladas por 4 parâmetros, $\alpha, \mu, \sigma$ e $\epsilon$ e são as seguintes:

- Escolha um par de sítios adjacentes (no que se segue, os estados desses sítios serão representados por um par $(X, Y)$, com a ordem determinada por um padrão, ou seja $(X, E)$ e $(E, X)$ representam a mesma aresta, mas se dizemos que $(X, E)$ vira $(X, Y)$, o sítio que tinha o estado $X$ permanece igual e o vazio adota o estado $Y$ ).

- Escolha uma de 3 possíveis interações, reprodução, competição e mobilidade. Essas interações são escolhidas com probabilidades

$$
\frac{\mu}{\mu+\sigma+\epsilon}, \frac{\sigma}{\mu+\sigma+\epsilon} \mathrm{e} \frac{\epsilon}{\mu+\sigma+\epsilon}
$$

respectivamente.

- Se a interação escolhida for reprodução e o estado do par for $(X, E)$, então o estado passa a ser $(X, X)$. Ou seja, durante a reprodução, se houver um sítio ocupado por uma espécie e um sítio vazio no par escolhido, então a espécie se espalha para o sítio vazio.

- Se a interação escolhida for mobilidade e o estado for $(X, Y)$, então o estado passa a ser $(Y, X)$. Ou seja, uma espécie se move para um espaço vazio sem se reproduzir (se $X$ ou $Y$ for igual a $E$ ), ou duas espécies trocam de lugar (se ambos forem diferentes de $E)$.

- Finalmente, se a interação escolhida for competição, um estado $(R, P)$ vira $(E, P)$ (com probabilidade 1), um estado $(P, S)$ vira $(E, S)$ (com probabilidade 1 ) e um estado $(S, R)$ vira $(E, R)$ com probabilidade $1-\alpha$ e permanece igual a $(S, R)$ caso contrário (probabilidade $\alpha$ ). Em todos os outros casos, nada acontece.

A assimetria é regida pelo parâmetro $\alpha$, com o modelo RPS usual sendo o caso $\alpha=0$. Esse modelo possui uma transição de fase na versão usual, quando variamos o parâmetro $\epsilon$ (usualmente são usados $\mu=\sigma=1$ ). O modelo sempre se organiza, porém de 2 formas diferentes. 
Para um valor suficientemente alto de $\epsilon$, o sistema vai para um dos estados absorventes (todos os sítios ou no estado $R$, ou no estado $S$, ou no estado $P$ ) e para valores baixos de $\epsilon$ temos a formação de um padrão robusto em que a rede é tomada por espirais (que podem ser explicadas pela equação de Landau-Guinzburg complexa), formando um estado quasi-periódico. Com a introdução de assimetrias, também temos uma transição de fase e para valores baixos de assimetria ( $\alpha \simeq 0.2$ ) também percebemos a formação dos mesmos padrões de espirais, porém com a formação de diversos padrões transientes antes deles se estabelecerem. Em particular, começando com uma condição incial em que as espécies são sorteadas com a mesma probabilidade e nenhum espaço vazio aparece inicialmente, obtemos padrões que lembram "bolhas". A medida que a assimetria aumenta (sempre usando essa mesma condição inicial), deixamos de ter espirais para ter estados em que as 3 espécies coexistem, mas com padrões espaciais complexos e com transientes em que o sistema passa por estados em que uma das espécies ocupa quase toda a rede. Alguns resultados preliminares também mostram que o valor de $\epsilon$ em que ocorre a transição para um dado tamanho de rede não escala da mesma forma com o tamanho do sistema.

Na figura 5.9.1, temos exemplos de alguns dos padrões espaciais que as espécies exibem. Essas simulações foram feitas em uma rede quadrada com lado $L=512, \mu=\sigma=1$. Diferentes valores de assimetria $(\alpha)$ foram usados e sempre com valores de mobilidade $(\epsilon)$ correspondentes a fase em que as espécies coexistem.

Finalmente, chamamos a atenção que esse modelo pode ser pensado também como um representante - com uma regra de confiança cíclica - da seguinte variante do modelo do votante, em que estados de indecisão são levados em conta:

- Escolha um sítio $i$ e um vizinho dele, $j$.

- Com certa probabilidade, $i$ e $j$ trocam de estado entre si. (mobilidade)

- Se não, $i$ tenta convencer $j$ :

- Se $i$ for indeciso nada acontece.

- Se não, caso $j$ seja indeciso, $i$ convence $j$. (reprodução)

- Se $j$ não for indeciso, então ele passa a ser indeciso com probabilidade $p_{\sigma_{j}, \sigma_{i}}$. (competição) 


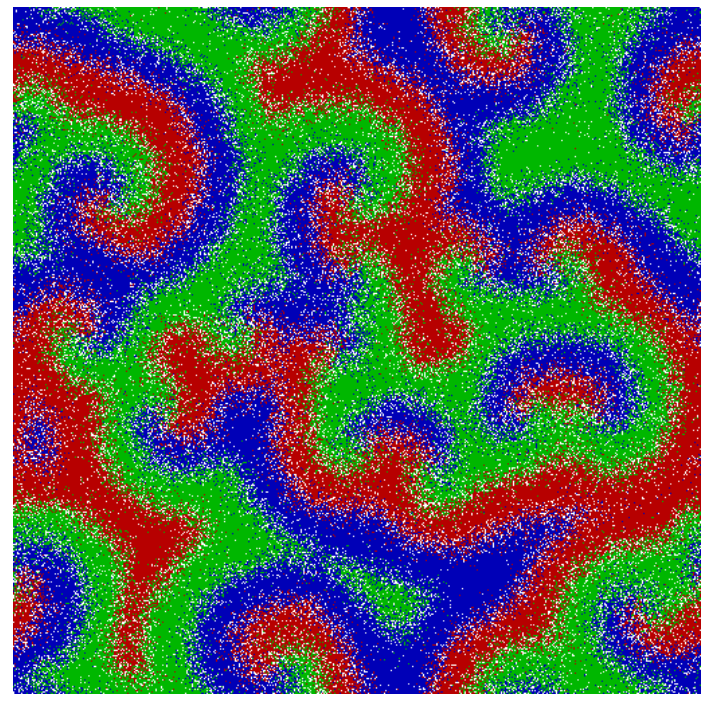

(a) $\alpha=0, \epsilon=25$

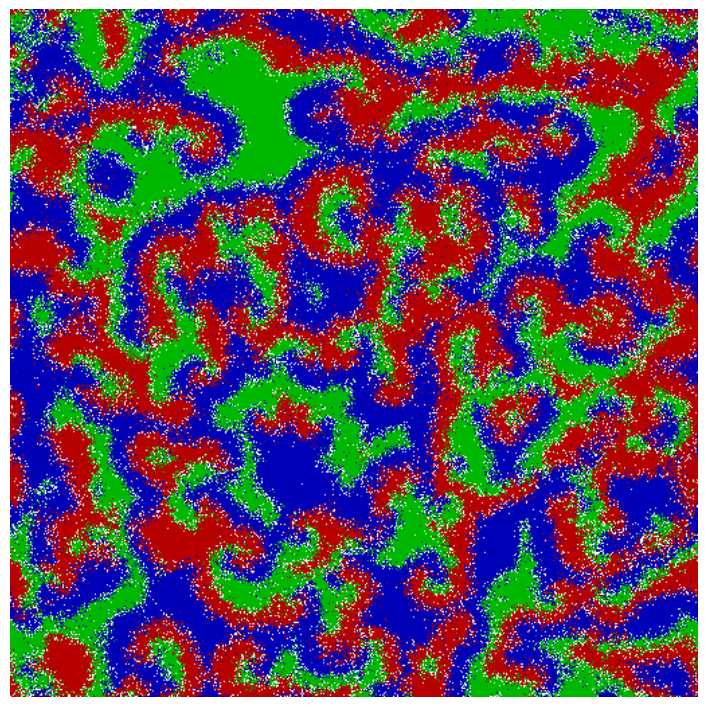

(c) $\alpha=0.4, \epsilon=3$

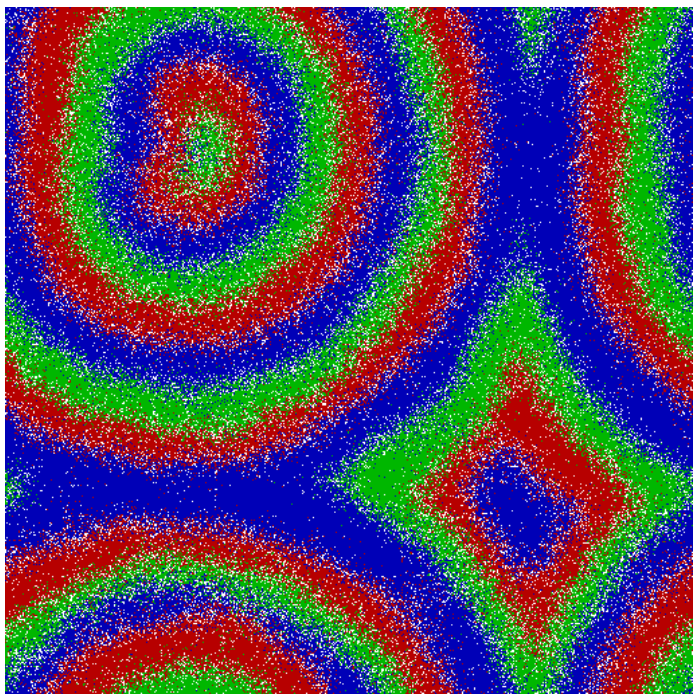

(b) $\alpha=0.1, \epsilon=25$

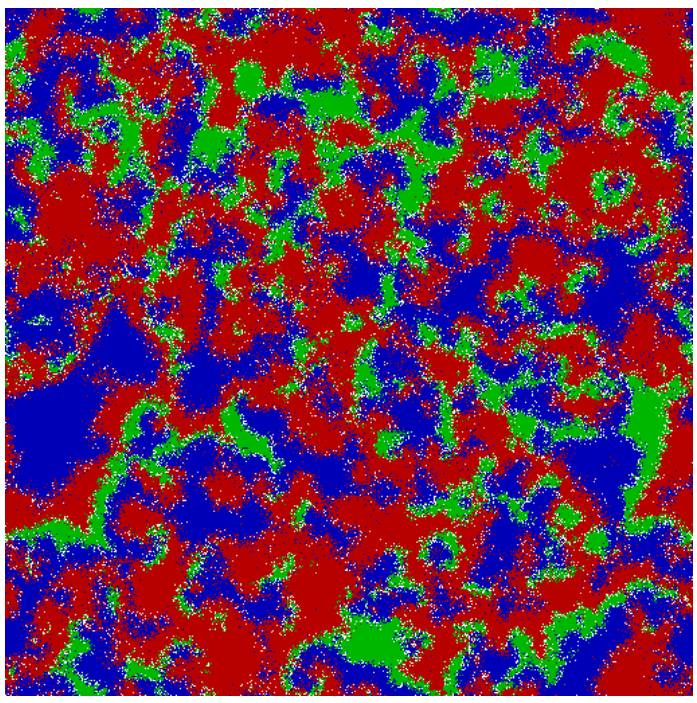

(d) $\alpha=0.7, \epsilon=1.5$

Figura 5.9.1. Padrões espaciais exibidos pelo modelo RPS, para diferentes valores de assimetria. Todas as simulações foram feitas em uma rede quadrada, com condições periódicas de contorno e lado $L=512$. As cores vermelha, verde e azul representam as espécies e o branco representa os espaços vazios. 


\section{Capítulo 6}

\section{Conclusão}

"It is not the answer that enlightens, but the question."

- Eugène Ionesco

Ao longo deste trabalho, estivemos preocupados em estudar o problema de como opiniões distintas conseguem coexistir em sociedades, uma vez que experimentos realizados por psicólogos ${ }^{[1 ; 36]}$ apontam que, em grupos pequenos, temos uma tendência a observar situações de consenso. Por causa da natureza complexa da interação entre seres humanos, é difícil obter resultados experimentais capazes de impor grandes restrições na modelagem desse fenômeno e, como consequência, existe uma míriade de modelos de propagação de opiniões distintos na literatura. Isso cria a priori um grande obstáculo, pois os resultados de um modelo podem não valer em outro e além disso, em modelos definidos em redes, a maioria deles tem uma forte dependência com a geometria da rede. O principal raciocínio por trás dessas modelagens é que o comportamento humano deve exibir características universais, de forma que os detalhes do modelo não devem ser importantes. Nesse espírito, e pensando em ajudar a conter a proliferação de modelos, propusemos uma abordagem capaz de unificar os modelos em diferentes famílias, as regras de confiança, que podem ser interpretadas como viéses e preconceitos (que são implementadas como conjuntos enormes de parâmetros) e nos propusemos a estudar o modelo do votante, o modelo Sznajd e suas generalizações. $\mathrm{O}$ aspecto qualitativo que esses modelos tem em comum é imitar alguns dos resultados experimentais obtidos por Asch na década de $50^{[1]}$, principalmente a eficiência desproporcional que uma maioria pode ter quando ela tenta convencer uma minoria (novamente, o experimento assume grupos pequenos). Para entender como essas regras poderiam funcionar, resolvemos estudar a aproximação de campo médio da equação mestra e chegamos na conclusão importante que não é necessário estudar o espaço de parâmetros inteiro e que todos as generalizações do modelo Sznajd se comportavam essencialmente da mesma forma (ambos esses fatos podem ser encarados como evidências da universalidade que esse tipo de modelagem assume que exista). 
Para obter esses resultados de campo médio, atribuímos a cada regra um grafo direcionado que chamamos de esqueleto da regra (a definição exata está na seção 2.1), em que os vértices são as opiniões e os arcos são as interações possíveis entre elas. Nós tratamos a aproximação de campo médio como um fluxo, em que as variáveis são as proporções em que cada opinião aparece no sistema. Fizemos um estudo do espaço de fase como um todo, significando que sorteamos as nossas condições iniciais aleatoriamente no espaço de fase. Essa abordagem tem a vantagem de estudar todas as estruturas presentes, o que evita o surgimento de discrepâncias que surgem quando sempre sorteamos todas as opiniões uniformemente na rede (como ocorre com o caso do modelo Sznajd com 3 opiniões e confiança limitada, que foi estudado na seção 5.6). Além disso, uma vez que os resultados são expressos como problemas de teoria de grafos temos um ganho tanto em termos de ferramental matemático, quanto de claridade e de intuição a respeito do comportamento da aproximação de campo médio, o que ajuda imensamente compreender as simulações.

Aplicando ferramentas de sistemas dinâmicos para estudar os pontos fixos do modelo na ausência de ruído chegamos nas seguintes conclusões:

- Todos os pontos fixos e suas propriedades de estabilidade podem ser determinadas a partir do esqueleto $\mathcal{R}$ da regra de confiança.

- Existe um ponto fixo em que as opiniões $\Delta$ coexistem se e somente se o grafo $\mathcal{R}_{\Delta}$, induzido por esse conjunto, for uma união de componentes fortemente conexas.

- Se $\mathcal{R}$ tiver apenas uma componente e ela for fortemente conexa, então existe exatamente um ponto fixo em que todas as opiniões coexistem. Se o modelo tem mais de uma opinião, esse ponto é sempre um nó instável.

- Se $\mathcal{R}_{\Delta}$ induz as componentes $\Delta_{1}, \ldots, \Delta_{k}$ e $\mathcal{M}_{X}$ for a variedade em que as opiniões do conjunto $X$ são as que sobrevivem, então se $\vec{\eta}_{i}$ for um ponto fixo do modelo, que está na variedade $\mathcal{M}_{\Delta_{i}}$, segue que todas as combinações convexas dos $\vec{\eta}_{i}$ são pontos fixos do modelo.

- Como consequência, se $\Delta$ for independente, todos os pontos de $\mathcal{M}_{\Delta}$ são pontos fixos.

- Outra consequência é que se $\mathcal{R}_{\Delta}$ induz uma união de $k$ componentes fortemente conexas, então existe exatamente um hiperplano $k-1$ dimensional em que todas as opiniões coexistem. A menos que $\Delta$ seja independente, esse hiperplano é Lyapunov instável.

- Se $\Delta$ for o conjunto de opiniões que sobrevivem em um ponto fixo, $\Omega$ for o conjunto de opiniões que são extintas nele e $\sigma \in \Omega$, então a variedade $\eta_{\sigma}=0$ (aonde o ponto fixo está) é atrativa na vizinhança desse ponto fixo se e somente se existe alguma opinião em $\Delta$ capaz de convencer $\sigma$ (ou seja, o arco relevante está presente no esqueleto $\mathcal{R}$ ). 
- Uma consequência é que todos os atratores estáticos do modelo sem ruído são variedades $\mathcal{M}_{\Delta}$, aonde $\Delta$ é independente. Mais precisamente, $\mathcal{M}_{\Delta}$ é um atrator se e somente se $\bar{\Delta}=\Delta_{-}$(que implica que $\Delta$ é independente maximal).

- No caso geral, as propriedades de estabilidade de um dado ponto fixo só dependem de quais opiniões sobrevivem nele $(\Delta)$, quais são extintas $(\Omega)$ e como elas se relacionam na regra de confiança. Mais precisamente, se $\mathcal{R}_{\Delta}$ induz $k$ componentes, teremos

$-|\Delta|+\left|\left(\bar{\Delta}-\Delta_{-}\right) \cap \Delta_{+}\right|-k$ direções instáveis.

- $\left|\bar{\Delta} \cap \Delta_{-}\right|$direções estáveis.

- $k-1$ direções neutras, ao longo das quais não há movimento.

- $\left|\left(\bar{\Delta}-\Delta_{-}\right) \cap\left(\bar{\Delta}-\Delta_{+}\right)\right|$direções neutras, ao longo das quais há movimento, mas sem que a variedade seja atrativa, nem repulsiva (temos estabilidade Lyapunov, mas não estabilidade assintótica).

- Se uma regra $\mathcal{R}$ for tal que não existem atratores estáticos (não existem soluções para $\bar{\Delta}=\Delta_{-}$), então sempre temos pelo menos um ciclo heteroclínico. Esse cíclos conectam estados que representam consenso de determinadas opiniões, e correspondem a ciclos no esqueleto da regra, em que não temos arestas duplamente conectadas.

- Se um ciclo heteroclínico passa pelos estados de consenso de cada uma das opiniões de um conjunto $\Delta$, então $\mathcal{R}_{\Delta}$ possui um ciclo que passa por todos os seus vértices, de forma que o grafo é fortemente conexo e logo existe um ponto fixo instável no interior de $\mathcal{M}_{\Delta}$, que é a variedade em que o ciclo está confinado no espaço de fase.

- Uma consequência é que as trajetórias devem sempre ser ou atraídas para um dos atratores estáticos, ou atraídas para um desses ciclos (causando oscilações com período divergente). Note que mesmo em alguns casos em que temos um atrator estático, podemos ter ciclos heteroclínicos, que possuam uma bacia de atração.

Fizemos a dedução desses resultados para o modelo Sznajd e para as suas generalizações (apenas o modelo do votante, dentre os modelos estudados, não exibe esse cenário). Depois, fizemos simulações em redes Barabási-Albert para mostrar até que ponto os resultados de campo médio são válidos. Lembramos que para chegar na aproximação de campo médio, definimos as variáveis $\eta_{\sigma}$, que correspondem ao valor esperado para a proporção de sítios com opinião $\sigma$, dada uma distribuição de probabilidades para todos os $M^{N}$ estados possíveis da rede (onde $M$ é o número de opiniões e $N$ é o número de sítio) e depois assumimos que os sítios são "indistinguíveis", o que é aproximadamente equivalente a estudar o modelo em um grafo completo. Isso tem duas consequências, a primeira é que as previsões de campo médio se traduzem como médias sobre realizações do modelo na rede (isso é até certo ponto esperado, já que as equações de campo médio são deterministas, mas o modelo simulado é 
estocástico) e a segunda é que efeitos que dependam de correlações espaciais não são capturados pela aproximação. Na maior parte dos casos, o modelo na rede Barabási-Albert se comporta de maneira semelhante ao modelo de campo médio, como visto na seção 5.6, em que mostramos a excelente concordância para o caso de 3 opiniões. Porém algumas propriedades do transiente se tornam diferentes a medida que o número de opiniões cresce. Alguns estados estácionários que não são previstos como atratores aparecem para algumas regras com 5 opiniões (seção 5.4). Eles surgem em estados "próximos" de estados que correspondem a pontos fixos (no espaço de fase esses pontos fixos de que estamos falando estão sempre em alguma variedade $\mathcal{M}_{\Delta}$, em que $\Delta$ é independente), porém eles possuem alguma direção repulsiva (pois $\bar{\Delta} \neq \Delta_{-}$). Além disso, os estados contém opiniões que aparecem em quantidades pequenas, que são capazes (segundo a regra de confiança) de convencer alguma das opiniões que sobreviveram em uma quantidade maior e se isso acontecesse, o sistema seria direcionado para um atrator do campo médio. A principal causa desses "estados congelados" é a parte da regra de todos os modelos baseados no modelo Sznajd, que diz que um grupo (com mais de um agente) de agentes concordando entre si deve ser formado para que haja convencimento, de forma que se esses sítios estiverem isolados entre si, eles não conseguem levar o modelo para o estado estacionário previsto pelo campo médio. Outro efeito que contribui é explicado pelo próprio campo médio, pois a repulsão das trajetórias por essas variedades que não são atratores não é um efeito de primeira ordem (não é previsto pela análise de estabilidade linear). Mais precisamente, para a variante do modelo Sznajd em que é necessário um grupo de tamanho $q$, esse é um efeito de $q$-ésima ordem. Finalmente, dadas as origens desses estados estacionários, vemos que eles são uma espécie de efeito de tamanho finito e que essas situações congeladas devem voltar a se tornar instáveis com a adição de ruído. Podemos resumir as conclusões sobre as simulações que fizemos na rede Barabási-Albert e algumas de nossas especulações da seguinte forma:

- Grosso modo, o modelo na rede Barabási-Albert tem um comportamento de campo médio.

- As discrepâncias que observamos são causadas por sítios de uma opinião que se tornam isolados (o que não é considerado pelo campo médio).

- Essas discrepâncias devem ser acentuadas com o aumento do número de opiniões $M$ e com o aumento do tamanho $q$ dos grupos necessários, para que haja convencimento.

- Essas discrepâncias devem ser amenizadas com o aumento do tamanho da rede e com a adição de ruído (talvez completamente eliminadas, no caso da adição de ruído).

- Todos os estados estacionários observados estavam muito próximos de variedades $\mathcal{M}_{\Delta}$ em que $\Delta$ é independente (mesmo nos casos que não obedeciam ao campo médio).

- Apesar da tendência para discrepâncias maiores, quando o número de opiniões é maior, outros aspectos da regra de confiança devem ter algum papel. Por exemplo, ambas as 
regras das figuras 5.1.1 e 5.4.1 tem 5 opiniões, mas as discrepâncias só são observadas na última. Como a única forma de isolar sítios seria convencê-los, para que uma opinião $A$ se torne isolada é necessário alguma outra opinião $B$ que consiga convencer $A$ e de preferência tal que $A$ não consegue convencer $B$. Porém a opinião $A$ não é eliminada, de forma que deve existir uma opinião $C$, com a qual $A$ não interage e que consiga convencer todos os sítios $B$. Logo, especulamos que um pedaço do esqueleto do tipo $A \rightarrow B \rightarrow C$, sem conexões entre $A$ e $C$ deva ser necessário (porém não suficiente) para que as discrepâncias sejam observadas.

Desenvolvemos uma teoria perturbativa para incluir ruído na aproximação de campo médio. Obtivemos alguns resultados parciais que, acreditamos, indicam que as discrepâncias encontradas para o modelo na rede Barabási-Albert devem sumir nesse caso. Alguns desses resultados não dependem dos detalhes da regra de confiança usada, apenas de seu esqueleto, porém alguns aspectos do modelo com ruído podem depender de quais são os valores exatos dos parâmetros que estão sendo usado, assim como de aspectos do ruído usado. Resumidamente chegamos nos seguintes resultados:

- Se um ponto fixo possui alguma opinião $\sigma$, que não sobrevive nele $(\sigma \in \Omega)$ e que não pode ser convencida (pelas restrições da regra) por nenhuma das opiniões sobreviventes $(\Delta)$, então esse ponto fixo não sobrevive à adição de ruído.

- Uma consequência é que todos os pontos fixos dos ciclos heteroclínicos, exibidos pelo modelo sem ruído, são destruídos pela adição de ruído e esses ciclos viram ciclos limites ou variedades invariantes mais complexas.

- Se $\mathcal{R}_{\Delta}$ induz $k$ componentes e os pontos fixos da variedade $\mathcal{M}_{\Delta}$ obedecem a restrição imposta pelo primeiro item dessa lista, então esses pontos tem que obedecer a $k-1$ vínculos extras para que eles sobrevivam à adição de ruído. A forma exata desses vínculos é dada por equações algébricas não-lineares que dependem dos parâmetros da regra de confiança e do comportamento do ruído em $\mathcal{M}_{\Delta}$. Note que se $k=1$, esses pontos não necessitam dos vínculos extras. É possível mostrar também que pelo menos um ponto da variedade satisfaz todos os vínculos.

- Nossos resultados também nos levam a conjecturar que se $\mathcal{M}_{\Delta}$ é um atrator do modelo sem ruído, então pelo menos um ponto dessa variedade sobrevive como um atrator do modelo com ruído.

Finalmente, fizemos simulações do modelo Sznajd em redes que são qualitativamente diferentes tanto da rede Barabási-Albert, quanto da rede completa, que seria a equivalente do modelo de campo médio. Para essas simulações, utilizamos redes de Watts-Strogatz, construídas a partir de redes quadradas com condições periódicas de contorno. Utilizamos nesse caso uma regra de confiança com 4 opiniões que tem um caráter cíclico e que não 
tem atratores no campo médio. O que observamos é que quando usamos poucas reconexões (o que torna a rede mais próxima de uma rede quadrada) os resultados são completamente diferentes dos de campo médio. Algumas condições iniciais rapidamente vão para estados de consenso, que é o resultado esperado para o campo médio (entre 70\% e 90\% dependendo dos parâmetros da regra), porém as outras trajetórias são atraídas para o ponto em que todas as opiniões coexistem na mesma quantidade, ou seja, temos um atrator extra nesse caso. A medida que a quantidade de reconexões aumenta, esse atrator vira um ciclo limite e cresce até colidir com a borda. A quantidade de simulações que são atraídas para esse atrator inicialmente cresce e depois cai bruscamente, quando o atrator colide com a borda.

Para o modelo do votante, temos um cenário completamente diferente, na aproximação de campo médio. Os resultados principais são:

- As propriedades das trajetórias são inteiramente regidas por uma matriz antissimétrica $T$, calculada a partir dos parâmetros da regra de confiança.

- Quando essa matriz tem autovetores com todas as componentes positivas e autovalor 0 , então as trajetórias estão contidas em hipersuperfícies fechadas e podemos observar oscilações estáveis.

- O comportamento típico depende da paridade do número de opiniões. Quando o número de opiniões é par, a região do espaço de parâmetros em que temos oscilações tem medida nula, o que não acontece quando o número de opiniões é ímpar.

- Podemos escrever uma lei de conservação para cada autovetor de $T$ com autovalor nulo (essa é a origem dos comportamentos oscilatórios).

- Quasiperiodicidade é possível para todos as quantidades de opinião.

- Conjecturamos que o comportamento típico do sistema depende apenas do padrão de sinais da matriz $T$ (quais termos dela são negativos, nulos ou positivos).

Fizemos simulações do modelo na rede Barabási-Albert com 3 opiniões e com 4 opiniões. Para 4 opiniões observamos a quasiperiodicidade, prevista pelo campo médio, porém o mais interessante é que no caso com 3 opiniões, as oscilações do campo médio se tornam instáveis e o centro do espaço de fase torna o centro do espaço de fase repulsivo, levando o sistema para um estado de consenso. Esse comportamento pode ser comparado com o do modelo do votante com 3 opiniões e competição cíclica (cujo campo médio se enquadra na nossa abordagem e exibe oscilações do mesmo tipo) simulado em uma rede quadrada, aonde as oscilações se tornam instáveis, mas o centro do espaço de fase pode se tornar atrativo, levando o sistema a um estado de coexistência ${ }^{[41]}$.

O último modelo a ser estudado foi o modelo rock-paper-scissors de biodiversidade que tem algumas semelhanças com o modelo do votante com regras de confiança (esse é essencialmente o modelo do votante, porém um sítio que é convencido passa primeiro por um 
estado de indecisão). Estudamos esse modelo em um rede quadrada, com condições periódicas de contorno e chegamos a conclusão que mesmo com a adição de assimetrias (o que é um desvio de como esse modelo é normalmente estudado e se enquadra melhor em nossa idéia de regras de confiança), a coexistência das diferentes espécies depende essencialmente da mobilidade delas.

Grosso modo, percebemos o seguinte padrão em nossas simulações:

- As redes Barabási-Albert, completa, de Erdös-Rényi (grafo aleatório) e a rede de Watts-Strogatz com muitas conexões tendem a levar os modelos (todos os modelos que foram estudados nessas redes) para situações em que temos uma coexistência inativa de opiniões, ou seja, aonde somente opiniões que não interagem entre si permanecem na rede.

- A rede quadrada e a rede de Watts-Strogatz tendem a facilitar um coexistência ativa das opiniões, ou seja uma coexistência em que opiniões que interagem entre si permanecem na rede, sem atingir um estado estático.

- No modelo rock-paper-scissors, a mobilidade pode ser encarada como uma distorção da rede, que efetivamente torna as distâncias menores.

A conclusão principal que chegamos a partir dessas simulações e dos resultados de campo médio para os diversos modelos é que os comportamentos qualitativos dependem de alguns poucos aspectos qualitativos da regra de confiança (o esqueleto no caso das variantes do modelo Sznjad) e da geometria que está sendo usada. Em particular, interações cíclicas e redes em que os sítios estão relativamente distantes entre si parecem ser essenciais para o surgimento de coexistências ativas. O nosso trabalho pode ser encarado até certo ponto como uma avaliação do potencial desses modelos para explicar a existência de diversidade de opiniões em sociedades.

Como mencionamos no início dessa conclusão, esses resultados representam um ganho na intuição sobre como esses modelos funcionam e podemos sugerir algumas variantes que consideramos promissoras, baseados neles. Podemos levantar com esses resultados duas perguntas. A primeira é, quais regras de confiança com interações cíclicas correspondem a mecanismos de convencimento e de interações de opiniões, observados no mundo real (ou então, como montar um toy model convincente nesses moldes)? A segunda é, como conciliar a necessidade de distâncias grandes com os resultados experimentais sobre a estrutura de redes sociais? Uma sugestão de toy model para regras cíclicas, que podemos dar (inspirada pela regra da seção 5.5) é uma modelagem de pontos de vista otimistas e pessimistas em política. A idéia é que cada partido $X$ corresponde a duas opiniões $X_{P}$ (pessimista) e $X_{O}$ (otimista). Teriamos uma probabilidade para trocar de opinião dentro do partido (sendo mais fácil se tornar pessimista) e probabilidades para trocar de partido, sendo mais fácil se tornar otimista no novo partido e deixar um partido em uma situação de pessimismo. Para a segunda pergunta, especulamos que o problema é que as intensidades das diferentes interações sociais 
de cada sítio não são levadas em conta. É mais fácil ser convencido por pessoas com quem temos vínculos mais fortes e da mesma forma, essas interações são mais frequentes. Se essas intensidades forem levadas em conta, acreditamos que a medida de distância relevante para esses modelos pode não ser a distância média usual (seria necessário redefinir a noção de distância para atribuir uma distância que dependesse da intensidade das conexões usadas em um caminho). Isso está de acordo com teorias sociológicas, segundo as quais, a maioria dos links em uma rede social são "links fracos", ou seja, entre pessoas que não se conhecem tão bem ${ }^{[19 ; 20]}$. Um aspecto interessante é que essa estrutura das redes sociais é normalmente ignorada, mas poderia ser verificada experimentalmente.

Outra coisa a ser levada em conta é o efeito que o ruído pode ter. Apesar de não termos resultados tão precisos nesse caso, conseguimos criar a regra de confiança da figura 4.4.1, em que temos duas opiniões que só interagem mediadas pelo ruído (uma não convence a outra diretamente) e que exibe 2 estados atrativos, em que essas opiniões coexistem, mas uma delas mantém uma maioria (isso é um efeito não-trivial das outras opiniões da regra, que não sobrevivem no atrator). É possível argumentar pelo teorema da função implícita e pelos próprios resultados da seção 4.4.2 que se adicionarmos nessa regra uma probabilidade $p$, tal que $p_{3 \rightarrow 4}=p_{4 \rightarrow 3}=p \neq 0$, então devemos ter essa situação de quebra de simetria quando $p \ll \theta$, onde $\theta$ é a intensidade do ruído, e apenas um atrator simétrico quando $\theta \ll p$ com uma bifurcação (transição de fase) "entre essas duas situações". Chamamos a atenção que a adição de um ruído impede a formação de estados estáticos, de forma que qualquer situação em que opiniões coexistam e que seja robusta pela adição de um ruído é postencialmente importante.

Finalmente, gostariamos de registrar que os resultados da seção 5.6, junto com uma versão preliminar dos resultados da seção 4.2 já foram publicados ${ }^{[43]}$, assim como os resultados da seção $5.5^{[44]}$. Os resultados das seções 4.2, 4.3 e 4.5 foram enviados para publicação e um preprint está disponível eletronicamente ${ }^{[45]}$. Também foi publicado um artigo em colaboração com K. Sznajd-Weron e M. Tabiszewski, sobre o modelo Sznajd com independência ${ }^{[25]}$. Uma colaboração com C. Grebogi e A. P. S. de Moura (sobre o modelo rock-paper-scissors, apresentado na seção 5.9) está em andamento e foi iniciada em um estágio de 2 meses na Universidade de Aberdeen. Gostariamos de lembrar que a generalidade de grande parte do nosso tratamento indica que essa abordagem usando sistemas dinâmicos pode ser aplicada em modelos bem além da sociofísica, com as analogias entre o modelo rock-paper-scissors e o modelo do votante sendo apenas um exemplo. 


\section{Apêndice A}

\section{Teoremas utilizados}

"My friend G. H. Hardy, who was professor of pure mathematics, enjoyed this pleasure (in mathematical demonstrations) in a very high degree. He told me once that if he could find a proof that I was going to die in five minutes he would of course be sorry to lose me, but this sorrow would be quite outweighed by pleasure in the proof. I entirely sympathised with him and was not at all offended."

- Bertrand Russell

"Mathematics is the science of skillful operations with concepts and rules invented just for this purpose."

- Eugene Wigner

Nesse apêndice apresentamos alguns teoremas que foram utilizados ao longo do resto do trabalho. Todos os teoremas são apresentados com as suas provas, exceto os da seção A.4, que fogem do escopo de nosso trabalho. Os teoremas da seção A.1 (A.1.1, A.1.2 e A.1.3), assim como a noção de alcance e o teorema A.2.11 são contribuições nossas (até onde sabemos). A noção de $\mathrm{C}_{0}$-matriz é a definição de matriz fracamente diagonal dominante (weakly diagonal dominant), porém restrita a uma certa classe de matrizes (que é conhecida como $L_{0}$ ). Os outros teoremas são razoavelmente conhecidos, mas normalmente não aparecem acompanhados de suas provas. Em particular a seção A.3 explica como estender a teoria de perturbação (no espírito de uma teoria de perturbação em mecânica quântica não-relativística) para matrizes não-hermitianas, explorando algumas sutilezas que surgem, como por exemplo, as diferenças entre os autovetores à esquerda e à direita e a possibilidade da matriz que estamos perturbando ser não diagonalizável (por simplicidade, nós nos restringimos à primeira ordem da perturbação para os autovalores). Apesar dessa exposição ser razoavelmente comum em textos avançados de engenharia, é rara de ser encontrada em livros-texto voltados para físicos. 


\section{A.1 Teoremas sobre grafos}

Teorema A.1.1. Seja $G$ um grafo e $\Delta$ um conjunto de nós em $G$, tal que $\bar{\Delta}=\Delta_{-}$. Isso implica que $\Delta$ é um conjunto independente maximal.

Demonstração. Para ver isso, suponha que $\Delta_{-}=\bar{\Delta}$ mas que $\Delta$ não seja independente, segue então que existe $\sigma, \sigma^{\prime} \in \Delta$ tais que $\sigma \in\left\{\sigma^{\prime}\right\}_{+} \Leftrightarrow \sigma^{\prime} \in\{\sigma\}_{-} \Rightarrow \sigma^{\prime} \in \Delta \cap \Delta_{-} \neq \varnothing \Rightarrow$ $\Delta \cap \bar{\Delta} \neq \varnothing$, o que leva a uma contradição.

Logo se $\Delta_{-}=\bar{\Delta}$ então $\Delta$ é independente. Se $\bar{\Delta}=\varnothing$ então é trivial que $\Delta$ é maximal. Porém se $\bar{\Delta} \neq \varnothing$, podemos tomar $\sigma \in \bar{\Delta}$. De onde segue que

$$
\begin{gathered}
(\Delta \cup\{\sigma\})_{-}=\Delta_{-} \cup\{\sigma\}_{-}=\bar{\Delta} \cup\{\sigma\}_{-} \Rightarrow \\
\Rightarrow(\Delta \cup\{\sigma\}) \cap(\Delta \cup\{\sigma\})_{-}=(\Delta \cup\{\sigma\}) \cap\left(\bar{\Delta} \cup\{\sigma\}_{-}\right)= \\
=((\Delta \cup\{\sigma\}) \cap \bar{\Delta}) \cup\left((\Delta \cup\{\sigma\}) \cap\{\sigma\}_{-}\right) \supseteq \\
\supseteq(\Delta \cup\{\sigma\}) \cap \bar{\Delta}=\{\sigma\} \neq \varnothing \Rightarrow(\Delta \cup\{\sigma\}) \cap(\Delta \cup\{\sigma\})_{-} \neq \varnothing,
\end{gathered}
$$

o que implica que $\Delta \cup\{\sigma\}$ não é independente e logo que $\Delta$ é maximal.

Def A.1.1. Um grafo direcionado $G$ é dito acíclico se não existem ciclos nele (uma aresta duplamente conectada conta como um ciclo para os efeitos dessa definição)

Def A.1.2. Um ordenamento topológico de um grafo direcionado $G$ é um ordenamento parcial estrito, $\prec$, tal que $i \prec j$ sss existe um caminho de $j$ até $i$ em $G$. Um grafo direcionado possui ordenamentos desse tipo sss ele for acíclico (o ordenamento pode ser construído com o algoritmo de $\mathrm{Kahn}^{[24]}$ ou de Tarjan ${ }^{[42]}$ ).

Teorema A.1.2. Seja $G$ um grafo direcionado tal que nenhum conjunto de nós obedece $\bar{\Delta}=$ $\Delta_{-}$, então existe um ciclo direcionado em $G$ que não usa nenhuma das arestas duplamente conectadas.

Demonstração. Suponha que não exista tal ciclo em $G$ e seja $G^{\prime}$ o grafo $G$ após a remoção de todas as arestas duplamente conectadas. Por hipótese, $G^{\prime}$ é um grafo direcionado acíclico e logo um ordenamento topológico em $G^{\prime}$ é possível. Ou seja, podemos definir um ordenamento parcial estrito em $V(G)$ :

$$
i \prec j \text { sss existe um caminho de } j \text { até } i \text { em } G^{\prime} .
$$

Também podemos definir a restrição desse ordenamento a um subconjunto $\Omega$ de $V(G)$, de forma que $i, j \in \Omega \Rightarrow i \prec_{\Omega} j$ sss $i \prec j$ (note que isso não é a mesma coisa que dizer que existe um caminho em $G_{\Omega}^{\prime}$ ). Considere agora o conjunto $\Delta$ contruído pelo seguinte algoritmo:

1. Atribua $\Delta \leftarrow \varnothing, \Xi \leftarrow \varnothing$ e $\Omega \leftarrow V(G)$. 
2. Se $\Omega$ for igual a $\varnothing$ pare. Caso contrário, seja $i$ um elemento minimal de $\prec_{\Omega}$.

3. Remova $i$ de $\Omega$ e o adicione a $\Delta$.

4. Remova de $\Omega$ os elementos do predecessor de $i$ com respeito a $G$ que estão em $\Omega$, $\left(i_{-}(G) \cap \Omega\right)$ e adicione-os a $\Xi$.

5. volte para o passo 2 .

Por construção, o conjunto $\Xi$ obedece $\bar{\Delta}=\Xi \subseteq \Delta_{-}$(tomando o predecessor com respeito a $G$ ). Além disso, o conjunto $\Delta$ é independente. Para ver isso, suponha que em algum momento durante a construção de $\Delta$, não existam conexões em $G$ entre nós de $\Delta$ e nós de $\Omega$ quando chegamos no passo 2 (isso é trivialmente verdadeiro para a iteração inicial do algoritmo) e seja $i$ o elemento minimal de $\prec_{\Omega}$ escolhido nesse passo. Como $i$ é minimal, não existem nós em $\Omega$ tais que $j \prec_{\Omega} i$, logo não existem caminhos partindo de $i$ até qualquer outro elemento de $\Omega$ no grafo $G^{\prime}$ e portanto $i_{+}(G) \cap \Omega$ contém apenas nós que estão conectados com o $i$ através de arestas duplamente conectadas, implicando que $i_{+}(G) \cap \Omega \subseteq i_{-}(G) \cap \Omega$ e que no passo 4 nós estamos transferindo todos os nós de $\Omega$, que ainda tinham alguma conexão com $\Delta$ após o passo 3 , para o conjunto $\Xi$. Segue que após uma iteração do algoritmo,ainda não existem conexões entre nós em $\Delta$ e nós em $\Omega$ quando voltamos ao passo 2 na iteração seguinte (e logo por indução, isso vale durante toda a construção de $\Delta$ ). Mas como os nós são adicionados em $\Delta$ vindos de $\Omega$ um por vez, adicionar um nó novo não adiciona conexões entre elementos de $\Delta$, implicando que $\Delta$ permanece independente durante toda a construção. Por outro lado, isso implica que $\Delta_{-} \subseteq \bar{\Delta}$. Relembrando que pela construção de $\Xi$ nós temos $\bar{\Delta} \subseteq \Delta_{-}$, segue que $\Delta_{-}=\bar{\Delta}$.

A relevância desse teorema para os problemas que estudamos é que uma solução para $\bar{\Delta}=\Delta_{\text {- }}$ no esqueleto de uma regra de confiança é equivalente a um atrator estático no espaço de fase e dizer que o ciclo $\sigma_{1} \rightarrow \sigma_{2} \rightarrow \ldots \rightarrow \sigma_{1}$ em um esqueleto não possui arestas duplamente conectadas é equivalente a dizer que a curva poligonal $\overline{P_{\sigma_{1}} P_{\sigma_{2}} \ldots P_{\sigma_{1}}}$ é um ciclo heteroclínico no espaço de fase, significando que toda regra que não tem atratores deve ter pelo menos um ciclo heteroclínico. Além disso, como pode ser visto na seção 4.4.2, a adição de um ruído infinitesimal destrói todos os pontos fixos que fazem parte desse ciclo, deixando no lugar um ciclo limite ou uma outra variedade mais complexa (possivelmente, um atrator estranho).

Def A.1.3. $O$ alcance de um nó $i$, $i_{\text {span }}$ é o conjunto de todos os nós $j$ em $G$, tais que existe um caminho de $i$ até $j$. O alcance de um conjunto de nós, $\Delta$, é definido como a união dos alcances de cada um de seus nós.

Corolário 1. Todo alcance é um sorvedouro e se $\Delta$ for um sorvedouro então $\Delta=\Delta_{\text {span }}$.

Corolário 2. Como nenhum arco deixa um sorvedouro, se $X$ for um sorvedouro e $Y \subseteq X$, então $Y_{\text {span }}=Y_{\text {span }}\left(G_{X}\right)$, o alcance de $Y$ em $G_{X}$. 
Teorema A.1.3. Um sorvedouro (fonte) é minimal sss ele induz um grafo fortemente conexo.

Demonstração. Seja $\Delta$ um sorvedouro que induz um grafo fortemente conexo em $G$. Suponha por absurdo que $\Delta$ não seja minimal, então existe $\Gamma \subset \Delta$, tal que $\Gamma$ também é um sorvedouro e $\Gamma \neq \varnothing$. Seja $\omega \in \Delta-\Gamma$. Como $G_{\Delta}$ é fortemente conexo, então para todos $i, j \in \Delta$ temos $i \in j_{\text {span }}\left(G_{\Delta}\right)$ e $\operatorname{logo} \omega \in \Gamma_{\text {span }}\left(G_{\Delta}\right)$. Como $\Gamma \subset \Delta$ e $\Delta$ é um sorvedouro em $G$, segue que $\Gamma_{\text {span }}\left(G_{\Delta}\right)=\Gamma_{\text {span }}(G)$ e como $\Gamma$ também é um sorvedouro em $G$, temos $\Gamma_{\text {span }}(G)=\Gamma$. Mas isso implica que $\omega \in \Gamma$, o que é uma contradição e logo $\Delta$ deve ser minimal.

Por outro lado, seja $\Delta$ um sorvedouro minimal em $G$ e suponha por absurdo que $G_{\Delta}$ não seja fortemente conexo, então existem $i, j \in \Delta$ tais que $i \notin j_{\text {span }}\left(G_{\Delta}\right)$. $\Delta$ é um sorvedouro, $\operatorname{logo} j_{\text {span }}\left(G_{\Delta}\right)=j_{\text {span }}(G)$. Também, $j_{\text {span }}(G) \subseteq \Delta_{\text {span }}(G)=\Delta$. Portanto $j_{\text {span }}(G) \subseteq$ $\Delta-\{i\}$ é um subconjunto próprio de $\Delta$, não-nulo e que é um sorvedouro (pois ele é o alcance de um vértice), contradizendo a hipótese que $\Delta$ é minimal. Portanto, $G_{\Delta}$ deve ser fortemente conexo.

A prova para fontes é obtida considerando o grafo reverso $G^{T}$ e usando o caso para sorvedouros

A relevância para o nosso problema é que quando uma regra de confiança possui apenas uma componente, a condição para a existência de pontos fixos de coexistência é mais fácil de ser provada usando sorvedouros minimais, enquanto que a generalização para mais de uma componente é mais fácil de ser entendida usando a noção de componentes fortemente conectadas. Da mesma forma, é mais fácil verificar que um grafo é fortemente conexo (isso pode ser provado de maneira construtiva, por exemplo). Esse teorema faz a ponte entre as 2 maneiras de expressar essa condição de existência.

\section{A.2 Teoremas sobre matrizes}

Iremos nesse apêndice apresentar alguns conceitos de álgebra linear e da teoria qualitativa de matrizes. Vamos supor que o leitor está familiarizado com o conceito de determinantes, sistemas lineares e algumas operações básicas de matrizes como montar submatrizes, transposições e inverter matrizes.

Def A.2.1. $O$ posto de uma matriz $M$ é o maior valor $k$, tal que $M$ possui uma submatriz $S$, quadrada e de ordem $k$, tal que $\operatorname{det}(S) \neq 0$. Vamos denotar o posto de $M$ como $\operatorname{rk}(M)$.

Teorema A.2.1. $\operatorname{rk}(M)=\operatorname{rk}\left(M^{T}\right)$.

Demonstração. A prova é trivial. As submatrizes de $M^{T}$ são transpostas de submatrizes de $M$, de forma que como $\operatorname{det}\left(A^{T}\right)=\operatorname{det}(A)$, então existe uma submatriz de $M^{T}$ com ordem $k$ e determinante não-nulo se e somente se existe uma submatriz de $M$, com as mesmas propriedades. 
Teorema A.2.2. $M$ é uma matriz com posto $k$ se e somente se as suas colunas geram um espaço com dimensão $k$.

Demonstração. Isso é uma consequência do fato que se $Q$ é uma matriz quadrada de ordem $n$, então $\operatorname{det}(Q) \neq 0$ se e somente se as colunas de $Q$ forem linearmente independentes e $\operatorname{logo}$, se e somente se as colunas geram um espaço de dimensão $n$. $\operatorname{Se} \operatorname{rk}(M)=k$, então existe uma submatriz $S$ de $M$, com ordem $k$ e tal que $\operatorname{det}(S) \neq 0$. Logo as colunas de $M$ correspondentes à matriz $S$ são linearmente independentes e segue que a dimensão do espaço gerado pelas colunas de $M$ é pelo menos $k$. Porém, essa dimensão só poderia ser maior que $k$ se existissem pelo menos $k+1$ colunas linearmente independentes de $M$. Só que se essas colunas existissem, poderiamos usá-las para montar uma submatriz quadrada de $M$, com ordem pelo menos $k+1$ e que teria determinante não-nulo, contrariando a hipótese que o posto de $M$ é $k$.

Def A.2.2. Seja o sistema linear $A \cdot \vec{x}=\vec{b}$. Vamos chamar de matriz dos coeficientes $a$ matriz A e de matriz aumentada a matriz

$$
[A \mid \vec{b}] \text {. }
$$

Teorema A.2.3 (Rouché-Capelli). Um sistema linear possui soluções se e somente se os postos da matriz dos coeficientes $C$ e da matriz aumentada forem iguais. Além disso, se o sistema possuir soluções e ele tiver $n$ variáveis, então o conjunto das soluções forma um espaço afim (um espaço linear deslocado) com dimensão igual a $n-\operatorname{rk}(C)$.

Demonstração. Seja $A \cdot \vec{x}=\vec{b}$ o sistema linear em questão. Escrevendo esse sistema em termos das colunas $\vec{A}_{i}$ da matriz $A$ temos

$$
\sum_{i} x_{i} \vec{A}_{i}=\vec{b}
$$

Em outras palavras, o sistema possui soluções se e somente se $\vec{b}$ for uma combinação linear das colunas de $A$.

Suponha então que o sistema possua soluções e vamos denotar por $\left[\vec{y}_{1}, \ldots, \vec{y}_{k}\right]$ o espaço gerado pelos vetores $\vec{y}_{1}, \ldots, \vec{y}_{k}$. Como $\vec{b}$ é uma combinação linear das colunas de $A$, então $\vec{b} \in\left[\vec{A}_{1}, \ldots, \vec{A}_{n}\right]$ (note que o número de colunas de $A$ deve ser o número de variáveis) e logo $\left[\vec{A}_{1}, \ldots, \overrightarrow{A_{n}}\right]=\left[\overrightarrow{A_{1}}, \ldots, \overrightarrow{A_{n}}, \vec{b}\right]$. Porém os espaços nos 2 lados dessa equação são respectivamente o espaço gerado pelas colunas da matriz dos coeficientes e o gerado pelas colunas da matriz aumentada e pelo teorema A.2.2, isso significa que as 2 matrizes tem o mesmo posto.

Se por outro lado as duas matrizes tem o mesmo posto, isso significa que os espaços $\left[\vec{A}_{1}, \ldots, \overrightarrow{A_{n}}\right]$ e $\left[\vec{A}_{1}, \ldots, \overrightarrow{A_{n}}, \vec{b}\right]$ tem a mesma dimensão (novamente pelo teorema A.2.2). Porém, pela definição de espaço gerado, temos $\left[\vec{A}_{1}, \ldots, \vec{A}_{n}\right] \subseteq\left[\vec{A}_{1}, \ldots, \overrightarrow{A_{n}}, \vec{b}\right]$, de forma que 
os 2 espaços terem a mesma dimensão implica que eles são iguais. Como por definição $\vec{b} \in\left[\vec{A}_{1}, \ldots, \overrightarrow{A_{n}}, \vec{b}\right]$, então segue que $\vec{b} \in\left[\vec{A}_{1}, \ldots, \overrightarrow{A_{n}}\right]$ e logo $\vec{b}$ é uma combinação linear das colunas de $A$, implicando que o sistema linear possui soluções.

Finalmente, suponha que o posto da matriz dos coeficientes e da matriz aumentada seja $k$. Isso implica que o sistema linear possui apenas $k$ equações independentes (pois senão, poderiamos usar essas equações para montar uma submatriz quadrada da matriz aumentada, com determinante diferente de 0 e ordem maior que $k$ ). Também implica que existe uma submatriz de $A, S$, que é quadrada, com ordem $k$ e determinante não-nulo. Sem perda de generalidade, podemos supor que $S$ é a matriz formada pelas primeiras $k$ linhas e colunas de A:

$$
S=\left[\begin{array}{ccc}
a_{1,1} & \ldots & a_{1, k} \\
\vdots & \ddots & \vdots \\
a_{k, 1} & \ldots & a_{k, k}
\end{array}\right]
$$

O sistema pode então ser reescrito como

$$
\left\{\begin{array}{ccc}
a_{1,1} x_{1}+\ldots+a_{1, k} x_{k} & = & b_{1}-a_{1, k+1} x_{k+1}-\ldots-a_{1, n} x_{n} \\
\vdots & \vdots \\
a_{k, 1} x_{1}+\ldots+a_{k, k} x_{k} & = & b_{k}-a_{k, k+1} x_{k+1}-\ldots-a_{k, n} x_{n} .
\end{array}\right.
$$

Definindo então a matriz $B$, os vetores $\vec{x}_{l}$ e $\vec{x}_{v}$

$$
B=\left[\begin{array}{ccc}
a_{1, k+1} & \ldots & a_{1, n} \\
\vdots & \ddots & \vdots \\
a_{k, k+1} & \ldots & a_{k, n}
\end{array}\right] \quad, \vec{x}_{l}=\left[\begin{array}{c}
x_{k+1} \\
\vdots \\
x_{n}
\end{array}\right] \quad \text { e } \quad \vec{x}_{v}=\left[\begin{array}{c}
x_{1} \\
\vdots \\
x_{k}
\end{array}\right]
$$

podemos reescrever o sistema como

$$
S \cdot \vec{x}_{v}=\vec{b}-B \cdot \vec{x}_{l} \Rightarrow \vec{x}_{v}=S^{-1} \cdot \vec{b}-S^{-1} \cdot B \cdot \vec{x}_{l},
$$

de forma que as soluções do sistema são os vetores da forma

$$
\left[\begin{array}{c}
S^{-1} \cdot \vec{b}-S^{-1} \cdot B \cdot \vec{x}_{l} \\
\vec{x}_{l}
\end{array}\right]=\left[\begin{array}{c}
S^{-1} \cdot \vec{b} \\
\overrightarrow{0}
\end{array}\right]+\left[\begin{array}{c}
-S^{-1} \cdot B \\
\mathbb{1}
\end{array}\right] \cdot \vec{x}_{l},
$$

onde as coordenadas de $\vec{x}_{l}$ são variáveis livres. Isso implica que as soluções formam um espaço afim, com um numero de dimensões igual ao número de coordenadas de $\vec{x}_{l}$, que é $n-k$.

Def A.2.3. Seja $M$ uma matriz quadrada, então um autovetor à direita, com autovalor $\lambda$ é um vetor $\vec{x} \neq \overrightarrow{0}$ (ou, uma matriz coluna), tal que $M . \vec{x}=\lambda \vec{x}$. De forma análoga, um autovetor à esquerda, com autovalor $\lambda$ é um vetor $\vec{y} \neq \overrightarrow{0}$ (ou, uma matriz linha), tal que $\vec{y} \cdot M=\lambda \vec{y}$. 
Nessa definição, os autovalores são encarados como uma propriedade dos autovetores. Porém, como o próximo teorema deixa claro, é mais interessante pensar neles como sendo propriedades da matriz $M$.

Teorema A.2.4. Ambos os tipos de autovetores tem os mesmos autovalores e os autovalores de uma matriz $M$ são as raízes do polinômio característico $p_{M}(\lambda)=\operatorname{det}(\lambda \mathbb{1}-M)$.

Demonstração. Suponha que tenhamos uma matriz $M$ quadrada e com valores em $\mathbb{C}$ e um número complexo $\lambda$. Vamos estudar o seguinte sistema linear

$$
M . \vec{x}=\lambda \vec{x} \Leftrightarrow(\lambda \mathbb{1}-M) \cdot \vec{x}=\overrightarrow{0} .
$$

O vetor $\vec{x}$ é um autovetor à direita se e somente se ele resolve esse sistema e é tal que $\vec{x} \neq \overrightarrow{0}$. Como o sistema é homogêneo, existe um $\vec{x}$ satisfazendo essas propriedades se e somente se $\lambda \mathbb{1}-M$ tiver determinante nulo, ou seja, os únicos autovalores possíveis para autovetores à direita são as soluções $\operatorname{de} \operatorname{det}(\lambda \mathbb{1}-M)=0$, que é uma equação polinomial de grau igual a ordem da matriz $M$.

Suponha então que $\lambda$ seja um autovalor do autovetor à direita $\vec{x}$. Logo, $M . \vec{x}=\lambda \vec{x} \mathrm{e}$ calculando o transposto dos 2 lados da equação temos $\vec{x} \cdot M^{T}=\lambda \vec{x}$, ou seja, $\vec{x}$ é um autovetor à esquerda da transposta de $M$ e tem o mesmo autovalor que $\vec{x}$. Falta então apenas mostrar que $M$ e $M^{T}$ são tais que os seus autovetores à direita tem os mesmos autovalores. Isso segue do determinante de $(\lambda \mathbb{1}-M)$ ser igual ao de $(\lambda \mathbb{1}-M)^{T}=\left(\lambda \mathbb{1}-M^{T}\right)$, de forma que $M$ e $M^{T}$ tem o mesmo polinômio característico.

Def A.2.4. A multiplicidade algébrica de um autovalor $\lambda$, da matriz $M$, é a multiplicidade dele como uma raiz do polinômio característico $\operatorname{det}(\lambda \mathbb{1}-M)$. A multiplicidade geométrica é a dimensão do espaço gerado pelos autovetores com esse autovalor. Logo, pelo teorema A.2.3, essa multiplicidade é $n-\operatorname{rk}(\lambda \mathbb{1}-M)$, onde $n$ é a ordem de $M$. Isso também implica (pelo teorema A.2.1) que a multiplicidade geométrica pode ser calculada tanto pelos autovetores à esquerda, quanto pelos à direita.

Teorema A.2.5. A multiplicidade geométrica, de um autovalor, nunca excede a algébrica.

Demonstração. É possível mostrar que os coeficientes do polinômio característico de $M$ são somas de determinantes de submatrizes principais de $M$ (o procedimento para fazer essa expansão é explorado em mais detalhe na prova do teorema A.2.16, mais adiante), de forma que se os determinantes de todas as submatrizes principais, com ordem $k \neq 0$, forem nulos, o coeficiente multiplicando $\lambda^{n-k}$ será nulo.

Sabendo disso, seja $\lambda$ um autovalor de $M$. Então o 0 será autovalor de $\lambda \mathbb{1}-M$, com as mesmas multiplicidades, algébrica e geométrica. Segue que se o posto de $\lambda \mathbb{1}-M$ for $n-q$, então o 0 é um autovalor de $\lambda \mathbb{1}-M$, com multiplicidade geométrica $q$ e considerando o polinômio característico $\operatorname{det}(\mu \mathbb{1}-\lambda \mathbb{1}+M)$, em função de $\mu$, então os coeficientes multiplicando os termos $\mu^{q-1}, \ldots, \mu^{0}$ são com certeza nulos, implicando que o 0 tem uma 
multiplicidade algébrica de pelo menos $q$ (a matriz pode ter todas as submatrizes principais de ordem $n-q$ com determinante 0 , mesmo existindo submatrizes que não sejam principais com determinante não-nulo). Segue que o $\lambda$ deve ser um autovalor de $M$, com multiplicidade algébrica maior ou igual a multiplicidade geométrica.

Teorema A.2.6. Suponha que $\vec{u}$ seja um autovetor à esquerda de uma matriz $M$ e $\vec{v} e ́$ um autovetor à direita da mesma matriz. Se os autovalores de $\vec{u}$ e $\vec{v}$ forem distintos, então $\vec{u} \cdot \vec{v}=0$.

Demonstração. Por hipótese temos

$$
\vec{u} \cdot M=\lambda \vec{u} \quad \text { e } \quad M \cdot \vec{v}=\mu \vec{v},
$$

$\operatorname{com} \lambda \neq \mu$. Então

$$
\lambda \vec{u} \cdot \vec{v}=\vec{u} \cdot M \cdot \vec{v}=\mu \vec{u} \cdot \vec{v} \Rightarrow(\lambda-\mu) \vec{u} \cdot \vec{v}=0 \Rightarrow \vec{u} \cdot \vec{v}=0 .
$$

Podemos generalizar esse teorema no caso em que a matriz $M$ é diagonalizável, ou seja, tal que $M=$ P.D.P. $P^{-1}$, com $D$ diagonal ${ }^{1}$.

Teorema A.2.7. Seja $M$ uma matriz diagonalizável, então é possível montar duas bases $\left\{\vec{u}_{i}\right\}$ e $\left\{\vec{v}_{i}\right\}$, em que os $\vec{u}_{i}$ são autovetores à esquerda, os $\vec{v}_{i}$ são autovetores à direita, $\vec{u}_{i}$ e $\vec{v}_{i}$ tem o mesmo autovalor quando eles tiverem o mesmo índice $i$ e $\vec{u}_{i} \cdot \vec{v}_{j}=\delta_{i, j}$.

Demonstração. Como a matriz é diagonalizável, então $M=P . D \cdot P^{-1}$. Como $M . P=P . D$ e a matriz $D$ é diagonal, então ela deve conter os autovalores de $M$ e as colunas de $P$ devem ser todas autovetores à direita de $M$. Como $P$ é inversível, isso implica que esses vetores são linearmente independentes e podemos escolhê-los como a nossa base $\left\{\vec{v}_{i}\right\}$. Da mesma forma, $P^{-1} \cdot M=D \cdot P^{-1}$, de forma que as linhas $P^{-1}$ são autovetores à esquerda que também formam uma base, que será a nossa escolha para $\left\{\vec{u}_{i}\right\}$. Por definição, $\vec{u}_{i}$ e $\vec{v}_{i}$ tem como autovalor o $i$-ésmo termo da diagonal principal de $D$. Finalmente, $\vec{u}_{i} \cdot \vec{v}_{j}$ é igual ao termo da $i$-ésima linha da $j$-ésima coluna de $P^{-1} \cdot P$, de forma que $\vec{u}_{i} \cdot \vec{v}_{j}=\delta_{i, j}$.

$\mathrm{Na}$ verdade vale a seguinte generalização (que não iremos provar), mesmo no caso de uma matriz não-diagonalizável

Teorema A.2.8. Se $\lambda$ é um autovalor de uma matriz $M$, cujas multiplicidades algébrica e geométrica são iguais, então existem 2 bases $\left\{\vec{u}_{i}\right\}$ e $\left\{\vec{v}_{i}\right\}$, em que os $\vec{u}_{i}$ são autovetores à esquerda e os $\vec{v}_{i}$ são autovetores à direita, todos com autovalor $\lambda$. Além disso $\vec{u}_{i} \cdot \vec{v}_{j}=\delta_{i, j}$.

\footnotetext{
${ }^{1}$ Pode-se mostrar que uma matriz é diagonalizável se e somente se todas as multiplicidades algébricas e geométricas forem iguais e nesse caso, as colunas da matriz $P$ serão autovetores à direita de $M$, formando uma base do espaço inteiro. A idéia básica aparece na prova do teorema A.2.7.
} 
Def A.2.5. Uma matriz quadrada $M$ é positiva (negativa) definida se todos os seus autovalores tiverem a parte real positiva (negativa). Uma matriz positiva (negativa) semidefinida tem todos os seus autovalores com parte real não-negativa (não-positiva).

Def A.2.6. Uma permutação é um transformação que leva uma matriz quadrada de ordem $n, M=\left(m_{i, j}\right)$ em $M^{\prime}=\left(m_{i, j}^{\prime}\right)$, onde $m_{i, j}^{\prime}=m_{\phi(i), \phi(j)}$, onde $\phi$ é uma bijeção de $\{1, \ldots, n\}$ em $\{1, \ldots, n\}$. Toda permutação é uma transformação de similaridade usando $S=\left(\delta_{\phi(i), j}\right)$ como matriz de similaridade.

Def A.2.7. Uma matriz bloco triangular é uma matriz da forma

$$
\left[\begin{array}{cc}
A & B \\
0 & C
\end{array}\right] \text { ou da forma } \quad\left[\begin{array}{ll}
A & 0 \\
B & C
\end{array}\right] \text {. }
$$

Def A.2.8. Uma matriz irredutível é uma matriz em que nenhuma permutação a leva em uma matriz bloco triangular.

Def A.2.9. Uma matriz é uma $\mathbf{C}_{0}$-matriz sss ela for quadrada, real, todas as entradas fora da diagonal principal forem não-positivas e a soma de cada coluna for não-negativa (e logo todos os termos da diagonal principal são não-negativos).

No que segue iremos definir para todas as matrizes quadradas

$$
R_{i}(M)=\sum_{j \neq i}\left|m_{i, j}\right| \quad \text { e } \quad S_{i}(M)=\sum_{j} m_{i, j} .
$$

O $M$ será omitido se não houverem ambiguidades.

Teorema A.2.9. Seja $M$ uma matriz real quadrada invertível. Então $(-1)^{m} \operatorname{det}(M)>0$, onde $m$ é o número de autovalores reais negativos de $M$.

Demonstração. Como $M$ é real, segue que todos os coeficientes do seu polinômio característico são reais e logo as soluções dele que não são reais aparecem em pares conjugados. Como o determinante de uma matriz é o produto de todos os autovalores, então como $M$ é invertível segue que $\operatorname{det}(M)=N P C$, onde $N$ é o produto de todos os autovalores negativos de $M, P$ é o produto de todos os positivos e $C$ é o produto de todos os autovalores que não são reais (definindo esses produtos como 1 quando a matriz não tiver autovalores do tipo correspondente). $C$ é o produto de números positivos, pois podemos multiplicar esses autovalores aos pares, juntando $\lambda \operatorname{com} \bar{\lambda}$, de forma que $C>0$. Também é trivial que $P>0$ e que $(-1)^{m} N>0$, implicando que $(-1)^{m} \operatorname{det}(M)>0$.

Teorema A.2.10. Uma matriz hermitiana $H$ é positiva definida sss existe $S$ invertível, tal que $H=S S^{\dagger}$ 
Demonstração. Seja $H$ uma matriz hermitiana. Então $H$ pode ser decomposta em $H=$ $U D U^{\dagger}$, onde $U$ é unitária e $D$ é a matriz diagonal formada pelos autovalores de $H$. Como os autovalores de uma matriz hermitiana são reais, segue que se $H$ é positiva definida, então existe uma matriz real, diagonal e invertível $T$, tal que $D=T^{2}$ (por exemplo, a matriz diagonal formada pelas raízes quadradas dos autovalores de $H$ ). Logo, $H=U T^{2} U^{\dagger}=$ $(U T)(U T)^{\dagger}$. Logo se $S=U T$, segue que $H=S S^{\dagger}$. Além disso, a inversa de $S$ existe e é dada por $S^{-1}=T^{-1} U^{\dagger}$.

Se por outro lado, $H=S S^{\dagger}$, onde $S$ é invertível, então se $x$ for um autovetor de $H$ com norma 1 e autovalor $\lambda$, temos

$$
\lambda=\lambda x^{\dagger} x=x^{\dagger} H x=x^{\dagger} S S^{\dagger} x=y^{\dagger} y=\|y\|^{2},
$$

onde $y=S^{\dagger} x$. Como $S$ é invertível, $S^{\dagger} x=\overrightarrow{0} \Leftrightarrow x=\overrightarrow{0}$, logo $y \neq \overrightarrow{0} \Rightarrow \lambda>0$. Portanto $H$ é positiva definida.

O seguinte teorema é um fortalecimento que conseguimos provar, de um teorema encontrado em ${ }^{[23]}$ (a versão original dá o caso em que $\mu=0$ e $T$ é diagonal).

Teorema A.2.11. Seja $M$ uma matriz tal que $2 N=M+M^{\dagger}$ é positiva semidefinida e seja $\mu$ a multiplicidade do autovalor 0 para a matriz $N$. Então se $T$ é uma matriz hermitiana, positiva definida, então MT é sempre positiva semidefinida e tal que a soma das multiplicidades geométricas dos autovalores com parte real nula é menor ou igual a $\mu$.

Demonstração. Seja $2 N=M+M^{\dagger}$. Essa matriz é hermitiana e por hipótese positiva semidefinida, logo os seus autovalores são reais, não-negativos e os autovetores formam uma base ortonormal. Essa base pode ser dividida em 2 partes, $\left\{u_{i}\right\}$, que tem os autovetores com autovalor 0 e $\left\{v_{i}\right\}$, com todos os outros. Seja $\lambda_{i}>0$ o autovalor tal que $N v_{i}=\lambda_{i} v_{i}$ e $U$ o espaço vetorial gerado por $\left\{u_{i}\right\}$. Seja $x$ um vetor coluna e $x^{\dagger}$ o vetor linha complexo conjugado, então

$$
\begin{gathered}
x^{\prime}=\sum_{i} \alpha_{i} u_{i}, \quad x^{\prime \prime}=\sum_{j} \beta_{j} v_{j}, \quad \text { e } \quad x=x^{\prime}+x^{\prime \prime} \Rightarrow \\
x^{\dagger} N x=\left(x^{\prime \dagger}+x^{\prime \prime \dagger}\right) N\left(x^{\prime}+x^{\prime \prime}\right)=\left(N x^{\prime}\right)^{\dagger}\left(x^{\prime}+x^{\prime \prime}\right)+x^{\prime \prime \dagger}\left(N x^{\prime}\right)+x^{\prime \prime \dagger} N x^{\prime \prime}= \\
=x^{\prime \prime \dagger} N x^{\prime \prime}=\sum_{i, j} \beta_{i}^{*} \beta_{j} v_{i}^{\dagger} N v_{j}=\sum_{i, j} \beta_{i}^{*} \beta_{j} \lambda_{j} \delta_{i, j}=\sum_{i}\left|\beta_{i}\right|^{2} \lambda_{i} .
\end{gathered}
$$

Logo, $x^{\dagger} N x$ é maior que 0 se $x \notin U$ e 0 se $x \in U$. Por outro lado $2 \operatorname{Re}\left(x^{\dagger} M x\right)=x^{\dagger} M x+$ $\left(x^{\dagger} M x\right)^{*}=x^{\dagger}\left(M+M^{\dagger}\right) x=2 x^{\dagger} N x$. Seja $S$ uma matriz não-singular e $w$ um autovetor normalizado de $S^{\dagger} M S$, com autovalor $\gamma$. Tomando $x=S w$ segue

$$
\operatorname{Re}(\gamma)=\operatorname{Re}\left(\gamma w^{\dagger} w\right)=\operatorname{Re}\left(w^{\dagger} S^{\dagger} M S w\right)=\operatorname{Re}\left(x^{\dagger} M x\right)=x^{\dagger} N x .
$$


Logo, se $W$ é o subespaço $W=\{y \in W \Leftrightarrow S y \in U\}$, temos $\operatorname{Re}(\gamma)=0$ sss $w \in W$ e $\operatorname{Re}(\gamma)>0$ caso contrário. Como $\mu$ é a dimensão de $U$ e $S$ é não-singular, então $W$ tem dimensão $\mu$. Todos os autovetores de $S^{\dagger} M S$ correspondendo a autovalores com parte real nula estão em $W$, logo a soma das multiplicidades algébricas desses autovalores, que é a dimensão do espaço gerado pelos autovetores correspondentes é menor ou igual a $\mu$, uma vez que esse espaço gerado deve ser um subespaço de $W$.

Concluímos que $S^{\dagger} M S$ é positiva semidefinida e tal que a soma das multiplicidades geométricas dos autovalores com parte real nula é menor ou igual a $\mu$. Os autovalores de uma matriz, suas multiplicidades algébricas e geométricas, dependem apenas da forma canônica de Jordan da matriz em questão. Como a forma canônica é invariante por transformações de similaridade, se usarmos $S^{\dagger}$ como matriz de similaridade, vemos que $S^{\dagger} M S$ e $M S S^{\dagger}$ tem a mesma forma canônica e logo os seus espectros tem as mesmas propriedades. Finalmente, qualquer matriz hermitiana, positiva definida $T$ pode ser escrita como $T=S S^{\dagger}$ (com $\operatorname{det}(S) \neq 0$ ), como provado no teorema A.2.10.

Teorema A.2.12 (Gershgorin). Seja $M$ uma matriz quadrada com termo geral $m_{i j}$ e $C_{i} o$ círculo $\left\{z \in \mathbb{C}|| z-m_{i i} \mid \leq R_{i}(M)\right\}$. Então se $C$ for

$$
C=\bigcup_{i} C_{i}=\bigcup_{j} B_{j}
$$

onde $B_{j}$ é conexo e a união de $n_{j} C_{i}$ 's de forma que $B_{j} \cap B_{k}=\varnothing \forall j \neq k$, então existem $n_{j}$ autovalores de $M$ (contadas as multiplicidades) no conjunto $B_{j}$.

Demonstração. Se $\lambda$ é um autovalor de $M$ com autovetor $\vec{v}$, então seja $i$ tal que $\left|v_{i}\right|=$ $\max _{j}\left|v_{j}\right|$. Segue que $v_{i} \neq 0 \mathrm{e}$

$$
\begin{gathered}
M \vec{v}=\lambda \vec{v} \Rightarrow \sum_{j} m_{i j} v_{j}=\lambda v_{i} \Rightarrow v_{i}\left(\lambda-m_{i i}\right)=\sum_{j \neq i} m_{i j} v_{j} \Rightarrow \lambda-m_{i i}=\sum_{j \neq i} m_{i j} \frac{v_{j}}{v_{i}} \Rightarrow \\
\left|\lambda-m_{i i}\right|=\left|\sum_{j \neq i} m_{i j} \frac{v_{j}}{v_{i}}\right| \leq \sum_{j \neq i}\left|m_{i j}\right|\left|\frac{v_{j}}{v_{i}}\right| \leq \sum_{j \neq i}\left|m_{i j}\right|=R_{i} .
\end{gathered}
$$

Logo $\lambda \in C_{i}$ para algum $i$. Seja $D$ a matriz diagonal com a mesma diagonal principal que $M$ e seja $M(t)=t M+(1-t) D . M(t)$ tem a mesma diagonal principal e logo os centros dos círculos $C_{i}(t)$ não dependem de $t$ e os raios deles $R_{i}(t)$ são iguais a $|t| R_{i}$. Segue que se 2 círculos são disjuntos para $t=1$ eles são disjuntos para todo $t$ entre 0 e $1, \operatorname{logo}$ se $B_{j}$ é conexo, é a união de exatamente $n_{j}$ círculos e não intersecta com nenhum outro $B_{k}$, esses $n_{j}$ círculos não intersectam com nenhum dos outros $n-n_{j}$ círculos ( $n$ é a ordem de $M$ ) para nenhum $t$ entre 0 e 1 . Como cada círculo possui exatamente um autovalor para $t=0 \mathrm{e}$ os autovalores são funções contínuas dos elementos da matriz, então um autovalor só pode sair de um círculo e ir para outro se eles se intersectam (pois todo autovalor deve pertencer a algum círculo), mas então nesse caso os 2 círculos pertencem ao mesmo $B_{j}$ para $t=1$. 
Teorema A.2.13. Uma $C_{0}$-matriz é sempre positiva semidefinida e o 0 é o único autovalor com parte real nula possível.

Demonstração. Como

$$
R_{i}=\sum_{i \neq j}-m_{i, j}
$$

aplicando o teorema de Gershgorin, segue que se $\lambda$ é um autovalor de $M$, então existe um $i$ tal que $\left|\lambda-m_{i, i}\right| \leq R_{i} \Rightarrow \lambda-m_{i, i}=\rho e^{i \theta}$ onde $\rho \leq R_{i}$. Logo, $\operatorname{Re}(\lambda)=m_{i, i}+\rho \cos (\theta) \geq$ $m_{i, i}-\rho \geq m_{i, i}-R_{i}$. Por outro lado, da definição de $S_{i}$, segue $S_{i}=m_{i, i}-R_{i}$, logo $\operatorname{Re}(\lambda) \geq S_{i} \geq 0$. Mas se $\operatorname{Re}(\lambda)=0$, então $S_{i}=0, \rho=R_{i}$ e $\cos (\theta)=-1 \Rightarrow \operatorname{Im}(\lambda)=0$. Logo, se 0 não for um autovalor de $M$, segue que $M$ é positiva definida.

Teorema A.2.14. Seja $M$ uma $C_{0}$-matriz, irredutível e tal que $S_{i}>0$ para algum $i$, então $M$ é positiva definida.

Demonstração. Pelo teorema A.2.13, basta provar que $\operatorname{det}(M) \neq 0$. Por outro lado, se $S_{i} \neq$ $0 \forall i$, o teorema de Gershgorin implicaria que o 0 não é um autovalor (por um raciocínio análogo ao da prova do teorema A.2.13) e logo que $\operatorname{det}(M)$ seria diferente de 0 .

Como $M$ é irredutível, não existe nenhuma permutação que a leve em uma matrix bloco triangular, além disso permutações não modificam o determinante, logo podemos assumir sem perda de generalidade que $M$ é tal que existe um inteiro $r(M)$, tal que $S_{i}(M)>0$ se $i \leq r(M)$ e $S_{i}(M)=0$ se $i>r(M)$ (pois bastaria fazer uma permutação conveniente em $M)$. Considere agora a seguinte transformação em $M$ e suponha que $r(M)<n$.

Tome $i$ e $j$ tais que $i \leq r(M), j>r(M)$ e $m_{j, i} \neq 0$. Tal par sempre existe, pois senão $M$ seria bloco triangular. Pegue agora a linha $i$, multiplique por $-m_{j, i} / 2 m_{i, i}$ e adicione na linha $j$, para obter a matriz $M^{\prime}$. Os únicos termos de $M^{\prime}$ diferentes dos de $M$ são os da linha $j$, que obedecem

$$
m_{j, l}^{\prime}=m_{j, l}-\frac{m_{i, l} m_{j, i}}{2 m_{i, i}} .
$$

Se $l \neq i, j$, como o termo adicionado é menor ou igual a zero $\left(m_{i, l}, m_{j, i} \leq 0\right.$ e $m_{i, i}>0 \Rightarrow$ $\left.-m_{i, l} m_{j, i} / 2 m_{i, i} \leq 0\right)$, então $m_{j, l}^{\prime} \leq 0$ nesse caso. Além disso

$$
m_{j, i}^{\prime}=m_{j, i}-\frac{m_{i, i} m_{j, i}}{2 m_{i, i}}=\frac{m_{j, i}}{2}<0 .
$$

Finalmente, $m_{j, j}^{\prime}>0$, pois

$$
S_{j}\left(M^{\prime}\right)=-\sum_{l} \frac{m_{i, l} m_{j, i}}{2 m_{i, i}}=\frac{-S_{i}(M) m_{j, i}}{2 m_{i, i}}>0 \text { e } m_{j, j}^{\prime}=S_{j}\left(M^{\prime}\right)-\sum_{l \neq j} m_{j, l}^{\prime} .
$$

Uma consequência disso é que $m_{k, l}^{\prime}=0 \Rightarrow m_{k, l}=0$, de forma que $M^{\prime}$ é irredutível (pois qualquer permutação que a tornasse bloco triangular, também tornaria $M$ bloco triangular). 
Isso implica que a matriz $M^{\prime}$ obedece à todas as hipóteses do teorema, além disso $\operatorname{det}(M)=$ $\operatorname{det}\left(M^{\prime}\right)$. Fazendo uma permutação tal que $j \leftrightarrow r(M)+1$ vemos que $r\left(M^{\prime}\right)>r(M)$, de forma que podemos pegar a matriz $M$ original e aplicar essa transformação sucessivamente, até chegar em uma matriz $N$ tal que $r(N)=n$. Essa matriz seria tal que $\operatorname{det}(M)=\operatorname{det}(N)$ e $S_{i}(N)>0 \forall i$, o que como vimos implica $\operatorname{det}(N) \neq 0, \operatorname{logo} \operatorname{det}(M) \neq 0$, provando que $M$ é positiva definida.

Teorema A.2.15. Uma matriz quadrada simétrica irredutível em que pelo menos um dos termos da diagonal principal é não-nulo, possui submatrizes principais irredutíveis de todas as ordens.

Demonstração. O caso em que a ordem da submatriz é 1 segue trivialmente da hipótese que algum dos termos da diagonal principal é não-nulo.

Uma matriz ser irredutível, ou não, depende apenas de quais termos são nulos, ou não. Logo, basta provar o teorema para a matriz $M$ definida por

$$
m_{i, j}= \begin{cases}1, & \text { se } a_{i, j} \neq 0 \\ 0, & \text { se } a_{i, j}=0\end{cases}
$$

onde $a_{i, j}$ e $m_{i, j}$ são os termos de $A$ e $M$ respectivamente. Como $A$ é simétrica, segue que $M$, todas as submatrizes principais de $M$ e todas as suas permutações são simétricas. $M$ também é a matriz de adjacências de um grafo não-direcionado $G$, em que os vértices são os índices de $M$ (os inteiros entre 1 e $n$, a ordem da matriz $M$ ). Além disso, a matriz de adjacências do subgrafo induzido $G_{X}$ é a submatriz principal $M_{X}$, obtida mantendo apenas os índices em $X$. Como $M_{X}$ é simétrica, então se ela não for irredutível, existe uma permutação que leva ela em uma forma bloco diagonal. Como $X$ não é unitário, isso significa que

$$
M_{X}=\left[\begin{array}{cc}
M_{Y} & 0 \\
0 & M_{Z}
\end{array}\right],
$$

onde $X=Y \cup Z$, o que significa que $G_{X}=G_{Y} \cup G_{Z}$ e logo $G_{X}$ não é conectado. Basta então provar que se $G$ for conectado e com mais de um vértice, podemos remover algum dos vértices e obter um novo grafo conectado (o teorema seguiria por indução. Como esses grafos são equivalentes as submatrizes da matriz $A$ original, estariamos efetivamente construindo a matriz de ordem $n-1$, depois a de ordem $n-2$ e assim por diante, até encontrarmos a matriz de ordem 2).

Seja $i$, um vértice qualquer de $G$. Vamos definir os conjuntos $i_{(m)}$ pela relação de recorrência

$$
i_{(m+1)}=i_{(m)} \cup\left(i_{(m)}\right)_{+},
$$

onde $i_{(1)}=\{i\}$. Como $G$ é conectado e com $n$ vértices, então $i_{(n)}=V(G)$ e logo (como $G$ tem mais de um vértice), existe $m<n$, tal que 


$$
i_{(m)} \neq V(G) \quad \text { e } \quad i_{(m+1)}=V(G) .
$$

Logo, se $\delta=i_{(m+1)}-i_{(m)}$, então todos os vértices de $V(G)-\delta$ podem ser alcançados a partir de $i$ sem passar por vértices em $\delta$ e se $j \in \delta$, existe um caminho de $i$ a $j$, em que $j$ é o único vértice em $\delta$. Segue que se removermos um dos vértices em $\delta$, continuam existindo caminhos de $i$ até todos os outros vértices restantes no grafo. Logo, podemos remover qualquer um dos vértices de $\delta$, para obter um subgrafo induzido com ordem $n-1$, que é conectado.

Teorema A.2.16. Seja $M$ uma matriz quadrada e $M^{(\sigma)}$ a submatriz principal obtida após remover a linha e a coluna $\sigma$, então se

$$
\operatorname{det}(M)=0 \quad e \quad \sum_{i} \operatorname{det}\left(M^{(i)}\right) \neq 0,
$$

o 0 é um autovalor de $M$ com multiplicidade algébrica 1.

Demonstração. Como $\operatorname{det}(M)=0$, então trivialmente o 0 é um autovalor de $M$, logo a multiplicidade dele é 1 sss o coeficiente que multiplica o termo de $1^{\mathrm{a}}$ ordem em $\lambda$ no polinômio característico de $M, p_{M}(\lambda)$, for não-nulo. Para chegar nesse coeficiente, basta expandir o determinante que define $p_{M}$ em $2^{n}$ termos, usando a propriedade de multilinearidade. Essa expansão é feita separando primeiro os termos que vem de $M$ dos que vem de $\lambda \mathbb{1}$ na primeira linha, obtendo a soma de 2 determinantes, depois em cada um deles fazemos a mesma separação na segunda linha, para cada um dos 4 determinantes resultantes separamos as terceiras linhas e assim por diante. Depois, aplicamos a regra de Laplace para cada um dos determinantes em todas as linhas provenientes de $\lambda \mathbb{1}$. A expansão resultante expressa os coeficientes do polinômio como somas de determinantes de submatrizes principais. Para obter o termo de $1^{\text {a }}$ ordem olhamos os termos em que somente uma das linhas é proveniente de $\lambda \mathbb{1}$, ou seja, os determinantes

$$
\begin{gathered}
\left|\begin{array}{ccccc}
-\lambda & 0 & 0 & \ldots & 0 \\
m_{2,1} & m_{2,2} & m_{2,3} & \ldots & m_{2, n} \\
m_{3,1} & m_{3,2} & m_{3,3} & \ldots & m_{3, n} \\
\vdots & \vdots & \vdots & \ddots & \vdots \\
m_{n, 1} & m_{n, 2} & m_{n, 3} & \ldots & m_{n, n}
\end{array}\right|+\left|\begin{array}{ccccc}
m_{1,1} & m_{1,2} & m_{1,3} & \ldots & m_{1, n} \\
0 & -\lambda & 0 & \ldots & 0 \\
m_{3,1} & m_{3,2} & m_{3,3} & \ldots & m_{3, n} \\
\vdots & \vdots & \vdots & \ddots & \vdots \\
m_{n, 1} & m_{n, 2} & m_{n, 3} & \ldots & m_{n, n}
\end{array}\right|+\ldots= \\
-\lambda\left|\begin{array}{cccc}
m_{2,2} & m_{2,3} & \ldots & m_{2, n} \\
m_{3,2} & m_{3,3} & \ldots & m_{3, n} \\
\vdots & \vdots & \ddots & \vdots \\
m_{n, 2} & m_{n, 3} & \ldots & m_{n, n}
\end{array}\right|-\lambda\left|\begin{array}{cccc}
m_{1,1} & m_{1,3} & \ldots & m_{1, n} \\
m_{3,1} & m_{3,3} & \ldots & m_{3, n} \\
\vdots & \vdots & \ddots & \vdots \\
m_{n, 1} & m_{n, 3} & \ldots & m_{n, n}
\end{array}\right|+\ldots=
\end{gathered}
$$


Teorema A.2.17. Seja $M$ uma $C_{0}$-matriz simétrica, irredutível, tal que $S_{i} \equiv \sum_{j} m_{i, j}=$ $0 \forall i$. Então $M$ é positiva semidefinida e o 0 é autovalor com multiplicidade algébrica 1.

Demonstração. Isso é um corolário dos teoremas anteriores. Os teoremas A.2.13 e A.2.16, implicam que basta mostrar que

$$
\sum_{i} \operatorname{det}\left(M^{(i)}\right) \neq 0
$$

Como $M$ é irredutível, $\log$ para todo $i$ existe $j \neq i$ tal que $m_{j, i} \neq 0$. Por outro lado

$$
S_{k}\left(M^{(i)}\right)=-m_{k, i} \geq 0
$$

uma vez que $i$ não é um índice de $M^{(i)}$. Segue que $M^{(i)}$ é uma $C_{0}$-matriz e que além disso $S_{j}\left(M^{(i)}\right)>0$. Como $M^{(i)}$ é positiva semidefinida, $\operatorname{det}\left(M^{(i)}\right) \geq 0$ (teorema A.2.9). Pelo teorema A.2.14, basta encontrar um $i$ tal que $M^{(i)}$ seja irredutível, mas a existência dessa matriz é assegurada pelo teorema A.2.15.

\section{A.3 Derivadas de autovalores e teoria de perturbação em matrizes}

Seja $M$ uma matriz quadrada, tal que $\lambda$ é um autovalor com multiplicidade algébrica e geométrica iguais a $\mu$ (a condição na multiplicidade significa que a matriz $M$ é para os nossos propósitos diagonalizável). Então existem bases $\left\{\vec{u}_{i}\right\}$ e $\left\{\vec{v}_{i}\right\}$ de autovetores a esquerda e a direita respectivamente, todos com autovalor $\lambda$ e obedecendo $\vec{u}_{i} \cdot \vec{v}_{j}=\delta_{i, j}$. Suponha agora que adicionemos uma perturbação $\epsilon N$ de forma que os novos autovetores à esquerda de $M+\epsilon N$ no limite $\epsilon \rightarrow 0$ são da forma

$$
\vec{a}_{i}+\epsilon \vec{w}_{i}, \operatorname{com} \vec{a}_{i}=\sum_{j} \alpha_{i, j} \vec{u}_{j}
$$

e tal que o autovalor de $\vec{a}_{i}$ é $\lambda+\epsilon \mu_{i}$. Então no limite em que $\epsilon$ vai a zero, temos

$$
\begin{gathered}
\left(\vec{a}_{i}+\epsilon \vec{w}_{i}\right) \cdot(M+\epsilon N)=\left(\vec{a}_{i}+\epsilon \vec{w}_{i}\right)\left(\lambda+\epsilon \mu_{i}\right)+\mathcal{O}\left(\epsilon^{2}\right) \Rightarrow \\
\vec{a}_{i} \cdot M+\epsilon\left(\vec{w}_{i} \cdot M+\vec{a}_{i} \cdot N\right)=\lambda \vec{a}_{i}+\epsilon\left(\lambda \vec{w}_{i}+\mu_{i} \vec{a}_{i}\right)+\mathcal{O}\left(\epsilon^{2}\right) \Rightarrow \\
\vec{w}_{i} \cdot(M-\lambda \mathbb{1})=-\vec{a}_{i} \cdot\left(N-\mu_{i} \mathbb{1}\right) .
\end{gathered}
$$

Para cada autovetor $\vec{a}_{i}$, a equação A.3.2 dá um sistema linear com $\vec{w}_{i}$ e $\alpha_{i, j}$ como variáveis. Para cada autovetor à direita de $M, \vec{v}_{j}$ temos:

$$
0=\vec{w}_{i} \cdot(M-\lambda \mathbb{1}) \cdot \vec{v}_{j}=-\vec{a}_{i} \cdot\left(N-\mu_{i} \mathbb{1}\right) \cdot \vec{v}_{j} \Rightarrow \vec{a}_{i} \cdot N \cdot \vec{v}_{j}=\mu_{i} \vec{a}_{i} \cdot \vec{v}_{j} .
$$


Seja então a matriz $\mathfrak{N} \equiv\left(\vec{u}_{i} . N . \vec{v}_{j}\right)$ e $D$ a matriz diagonal com os valores de $\mu_{i}$ (ou seja as derivadas dos autovalores com respeito ao parâmetro de perturbação $\epsilon$ ) para cada autovetor $\vec{a}_{i}$ na diagonal principal, segue que

$$
A . \mathfrak{N}=D . A .
$$

Uma consequência é que todos os $\mu_{i}$ (e logo as derivadas dos autovalores iguais a $\lambda$ ) devem ser autovalores de $\mathfrak{N}$.

Para ver que os autovalores de $\mathfrak{N}$ dão todas as derivadas (e todo autovalor é alguma derivada), considere o caso em que $\mathfrak{N}$ é diagonalizável (a nossa hipótese sobre $M$ não garante isso). Então podemos resolver a equação A.3.3 usando uma matriz $A$ invertível. Se $A=$ $\left(\alpha_{i, j}\right)$, podemos usar esses coeficientes $\alpha_{i, j}$ para montar uma base de autovetores à esquerda de $M+\epsilon N$ que gera o mesmo espaço que a base $\left\{\vec{u}_{i}\right\}$ e $\vec{a}_{i}=\sum_{j} \alpha_{i, j} \vec{u}_{j}$ resolve A.3.1 (com a derivada do autovalor correspondente igual a $\mu_{i}$ ). Se a matriz $\mathfrak{N}$ for não-diagonalizável, então usamos uma matriz arbitrariamente próxima de $N$ para a perturbação e que seja tal que $\mathfrak{N}$ é diagonalizável (o resultado segue pois os autovalores são funções contínuas e logo estão arbitrariamente próximos dos valores que vem da perturbação original).

\section{A.4 Teoremas de Topologia e de Sistemas Dinâmicos}

Iremos nessa seção usar o conceito de índice da raiz de um campo vetorial $\vec{F}: \mathbb{R}^{n} \rightarrow \mathbb{R}^{n}$. A definição geral de índice foge do escopo desse trabalho, mas pode ser pensada como uma medida de multiplicidade da raiz, porém, não no sentido usual. Uma ilustração disso é a raiz que surge em um dipólo, que pode ser pensada como um encontro de 2 monopólos opostos (em termos de sistemas dinâmicos, um ponto fixo desse tipo pode ser o resultado da colisão de 2 nós, um estável e o outro instável) e logo seria intuitivo que ele tivesse multiplicidade (e logo índice) 2. Porém o índice é uma multiplicidade no sentido que o índice do dipólo é a soma dos índices de cada um dos nós que colidiram para formá-lo, o que dá um resultado $1+(-1)^{n}$ (isso pode ser visto usando a definição simplificada que vamos fornecer), que só dá 2 se o número de dimensões for par. A definição que iremos usar vale para raízes em que o jacobiano $\mathcal{J}$ do campo é inversível quando avaliado na raiz que queremos calcular o índice.

Def A.4.1. Seja $\vec{x}^{*}$ a raiz de um campo vetorial $\vec{F}: \mathbb{R}^{n} \rightarrow \mathbb{R}^{n}$ e $\mathcal{J}$ o jacobiano de $\vec{F}$, avaliado em $\vec{x}^{*}$, então se $\operatorname{det}(\mathcal{J}) \neq 0$, segue que o índice é dado por

$$
\left\{\begin{aligned}
-1 & , \text { se } \operatorname{det}(\mathcal{J})<0 \\
1 & \text {, se } \operatorname{det}(\mathcal{J})>0
\end{aligned}\right.
$$

Chamamos a atenção que no caso geral, o índice pode a priori assumir qualquer valor inteiro e se referir a variedades mais gerais. 
Para o próximo teorema iremos usar também a característica de Euler, $\chi$. Como não iremos usar variedades complicadas, não vale a pena fazer a definição rigorosa desse invariante topológico. Basta saber que se uma variedade pode ser deformada continuamente (ou seja, através de homeomorfismos) em um ponto, então o invariante dela é 1.

Teorema A.4.1 (Poincaré-Hopf). Seja $\mathcal{M}$ uma variedade compacta e diferenciável, com um campo vetorial diferenciável, $\vec{v}$, definido nela, que só possui raízes isoladas (toda raiz tem uma vizinhança aberta em que ela é única). Se $\mathcal{M}$ não tiver borda, ou se $\vec{v}$ aponta para fora ao longo de toda a borda, então a soma dos índices das raízes de $\vec{v}$ em $\mathcal{M}$ é $\chi_{\mathcal{M}}$.

Esse teorema também pode ser pensado como uma forma de calcular a característica de Euler de uma variedade, tentando definir nela um campo bem comportado e que obedeça às hipóteses, para depois calcular a soma dos índices.

Teorema A.4.2 (Hartman-Grobman). Seja um campo vetorial suave $\vec{F}: \mathbb{R}^{n} \rightarrow \mathbb{R}^{n}$ e a equação diferencial $\dot{\vec{x}}=\vec{F}(\vec{x})$. Se $\vec{x}^{*}$ for uma raiz e $\mathcal{J}$ for o jacobiano de $\vec{F}$ avaliado em $\vec{x}^{*}$, então se todos os autovalores tem parte real não nula, as soluções da equação diferencial na vizinhança de $\vec{x}^{*}$ são topologicamente equivalentes às da equação $\dot{\vec{x}}=\mathcal{J} \cdot\left(\vec{x}-\vec{x}^{*}\right)$.

Esse teorema fornece condições para que a linearização do sistema linear dê uma descrição fiel do comportamento ao redor dos pontos fixos de um fluxo e é o que embasa todas as técnicas de análise de estabilidade linear. Um detalhe interessante é que ele mostra que esse tipo de análise não é suficiente para encontrar centros.

O seguinte teorema pode ser usado como um ponto de partida para estudar a estabilidade ao longo das direções em que a parte real do autovalor correspondente é nula (a análise desses casos é conhecida na literatura como teoria da variedade central). O enunciado que iremos dar assume, por simplicidade, que o jacobiano é diagonalizável, porém o teorema poderia ser generalizado, escrevendo o jacobiano na forma canônica de Jordan dele.

Teorema A.4.3 (Variedade Central). Seja o fluxo $\dot{\vec{x}}=\vec{F}(\vec{x})$, onde $\vec{F}: \mathbb{R}^{n} \rightarrow \mathbb{R}^{n}$ é r vezes continuamente diferencíavel $\left(C^{r}\right)$. Se $\vec{x}^{*}$ for um ponto fixo e $\mathcal{J}$ for o jacobiano de $\vec{F}$, então temos 3 subespaços afins $W_{e}, W_{i}$ e $W_{c}$, gerados respectivamente pelos autovetores de $\mathcal{J}$ que tem autovalores com parte real negativa, positiva e nula e deslocando a origem desses espaços para $\vec{x}^{*}$. Esses 3 subespaços de $\mathbb{R}^{n}$ são chamados de subespaços estável, instável e central, respectivamente. Para cada um desses espaços, temos variedades formadas inteiramente por trajetórias do fluxo e que são tangentes ao subespaço no ponto $\vec{x}^{*}$. As variedades tangentes a $W_{e}, W_{i}$ e $W_{c}$ são chamadas respectivamente de variedades estável, instável e central e elas possuem as seguintes propriedades:

- Tanto a variedade instável, quanto a estável, são únicas, $r$ vezes continuamente diferenciáveis e com o mesmo número de dimensões que $W_{i}$ e $W_{e}$, respectivamente.

- A variedade central não é necessariamente única, é $r-1$ vezes continuamente diferenciável e com o mesmo número de dimensões que $W_{c}$. 



\section{Apêndice B}

\section{Códigos utilizados}

"If it's not on fire, it's a software problem."

- Anônimo

"On two occasions I have been asked (by members of Parliament): 'Pray, Mr. Babbage, if you put into the machine wrong figures, will the right answers come out?' I am not able rightly to apprehend the kind of confusion of ideas that could provoke such a question."

- Charles Babbage

Este apêndice contém para fins de referência a parte principal do código usado para fazer as simulações na rede (sznajd_base.c. Algumas das versões de fato usadas tinham partes adicionais para fazer diferentes medidas, mas todas eram baseadas no código aqui apresentado) e o código usado (de fato) para gerar as redes Barabási (cria_BA.c) que foram usadas nas simulações. Para integrar as equações de campo médio, foi usado um método Runge-Kutta adaptativo, fornecido no pacote SciPy ${ }^{[35]}$ (o uso de um método adaptativo é crucial para as regras que exibem transientes longos, mas dispensável nos outros casos). Também é fornecida a documentação dos formatos de arquivo usados nesses códigos, além de instruções para modificar o código do modelo Sznajd, para simular o modelo do votante. Não estão inclusos os códigos utlizados para fazer a simulação do modelo rockpaper-scissors.

Utilizamos para fazer as simulações o cluster abax do grupo de mecânica estatística do departamento de física geral do Instituto de Física da Universidade de São Paulo. Para dar uma idéia dos custos computacionais envolvidos, uma simulação (s znajd_base.c) com duração de 1000 tempos de Monte Carlo em uma rede Barabási-Albert de tamanho $N=10^{6}$ e conectividade mínima $m=5$, usando a regra estudada na seção 5.1 e condição inicial sorteada usando $\vec{\eta}=\overrightarrow{1} / 5$, simulada em uma CPU Pentium P6200 $(2.13 \mathrm{GHz}, 3 \mathrm{Mb}$ de cache, 64 bits) com 3,3Gb de memória RAM disponível (sem swap) demandou 66Mb de memória e demorou 10min30s. O arquivo de saída resultante teve 36kb de tamanho. Para uma 
simulação de 100 tempos de Monte Carlo a duração da simulação é de 62s e o arquivo de saída teve $34 \mathrm{~kb}$ de tamanho. Para gerar uma rede Barabási-Albert com tamanho $N=10^{7} \mathrm{e}$ $m=5$ nas mesmas condições foi utilizado $2,7 \mathrm{~Gb}$ de memória e a criação da rede demorou $82 \mathrm{~s}$. O arquivo contendo a rede teve $750 \mathrm{Mb}$ de tamanho. Para gerar uma rede $N=10^{6}$ foi utilizado $270 \mathrm{Mb}$ de memória, levou $6 \mathrm{~s}$ e o arquivo de saída tinha $69 \mathrm{Mb}$. Uma rede de tamanho $N=10^{5}$ foi construída em menos de $1 \mathrm{~s}$, com $27 \mathrm{Mb}$ de memória e um arquivo de saída com 5,6Mb.

\section{B.1 Formatos dos arquivos utilizados}

Os códigos desse capítulo usam 4 formatos diferentes de arquivo para diferentes funções. Arquivos de regra de confiança (.p) que contém os parâmetros da regra a ser usada, arquivos de geometria (.geo) que contém uma descrição da rede usada como substrato da simulação (em uma representação de sítios), arquivos para representar o estado microscópico (. initial e .output) que são usados para especificar a opinião de cada sítio (tanto na condição inicial, quanto para armazenar o estado final em alguns casos) e arquivos para representar o estado macroscópico do sistema (. eta) ao longo do tempo, ou seja, as trajetórias no espaço de fase.

\section{B.1.1 Arquivos de regra de confiança}

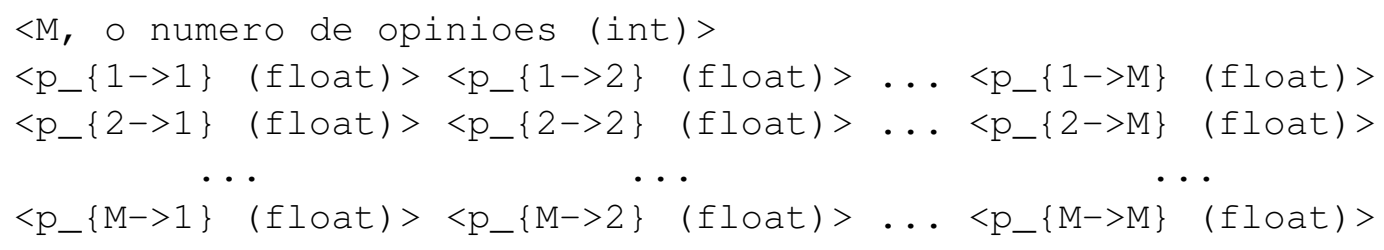

\section{B.1.2 Arquivos de geometria}

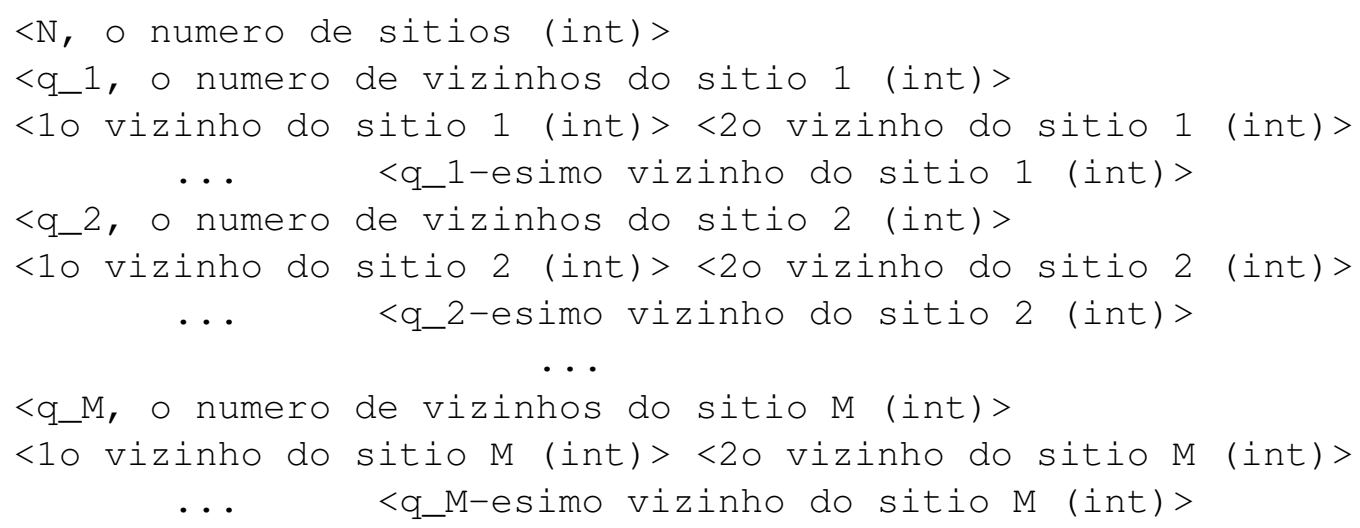




\section{B.1.3 Arquivos de condição inicial e de saída (estado microscópico)}

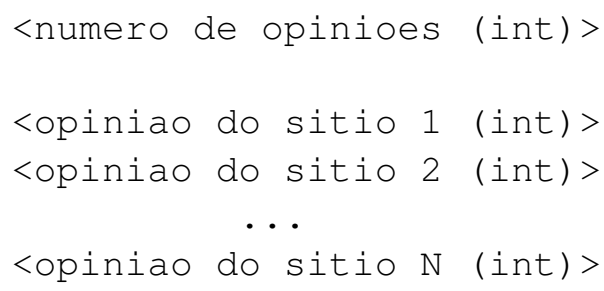

\section{B.1.4 Arquivos de saída (estado macroscópico)}

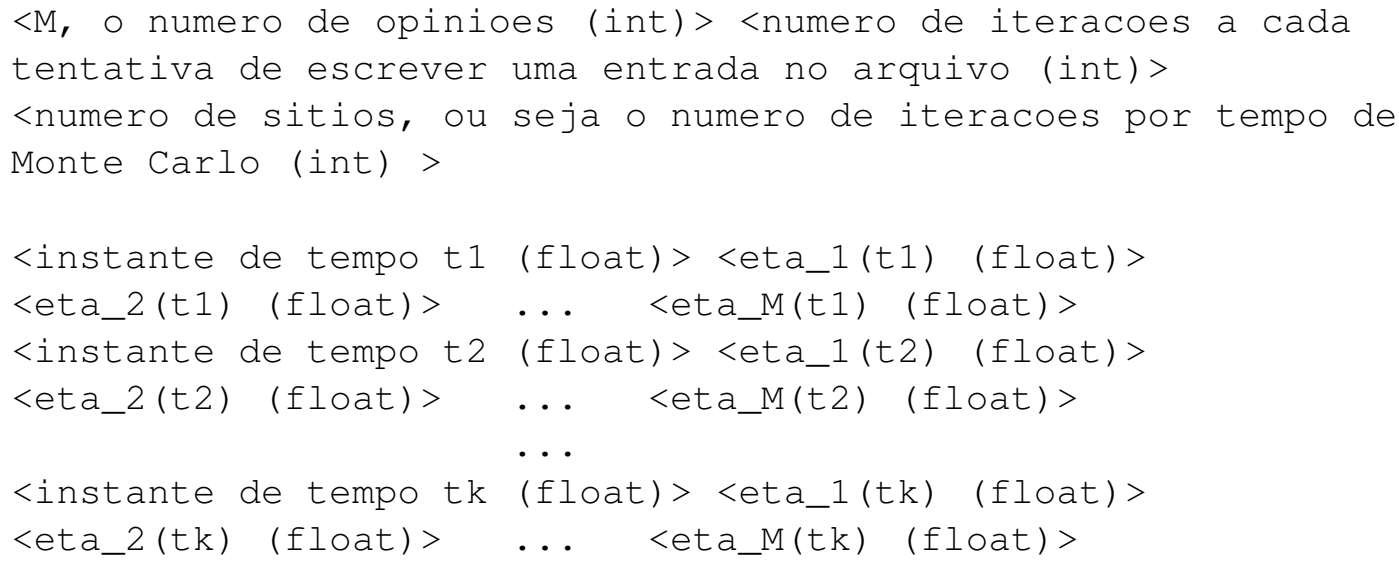

\section{B.2 sznajd_base.c}

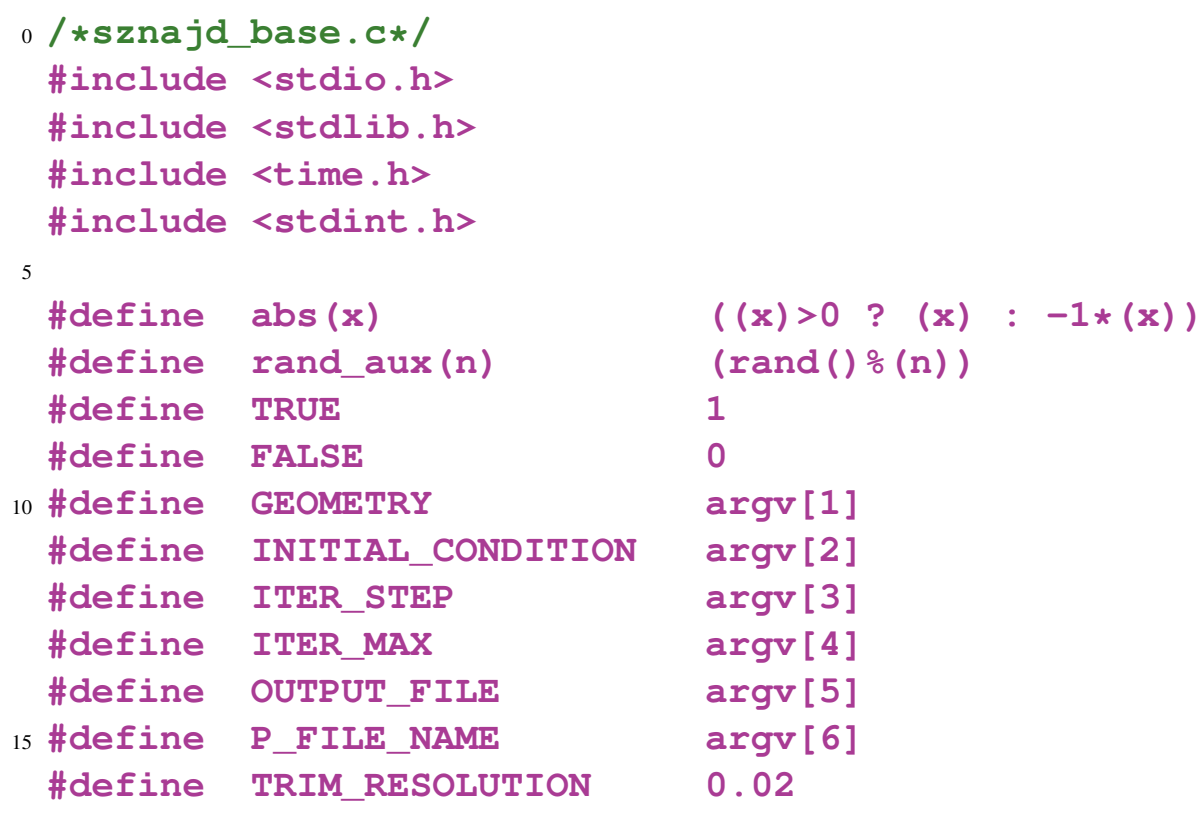




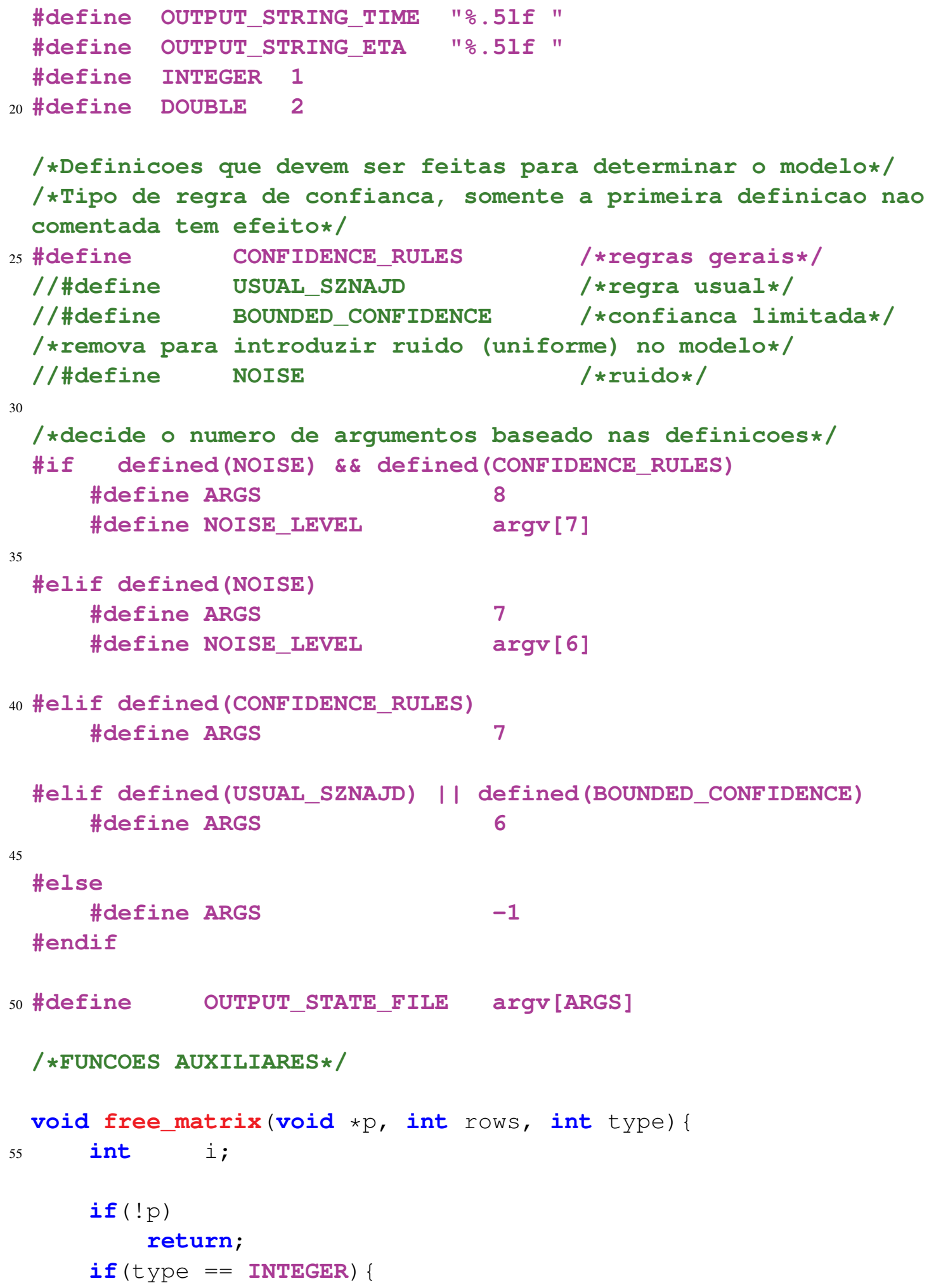


60

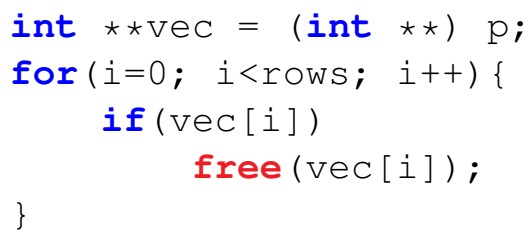




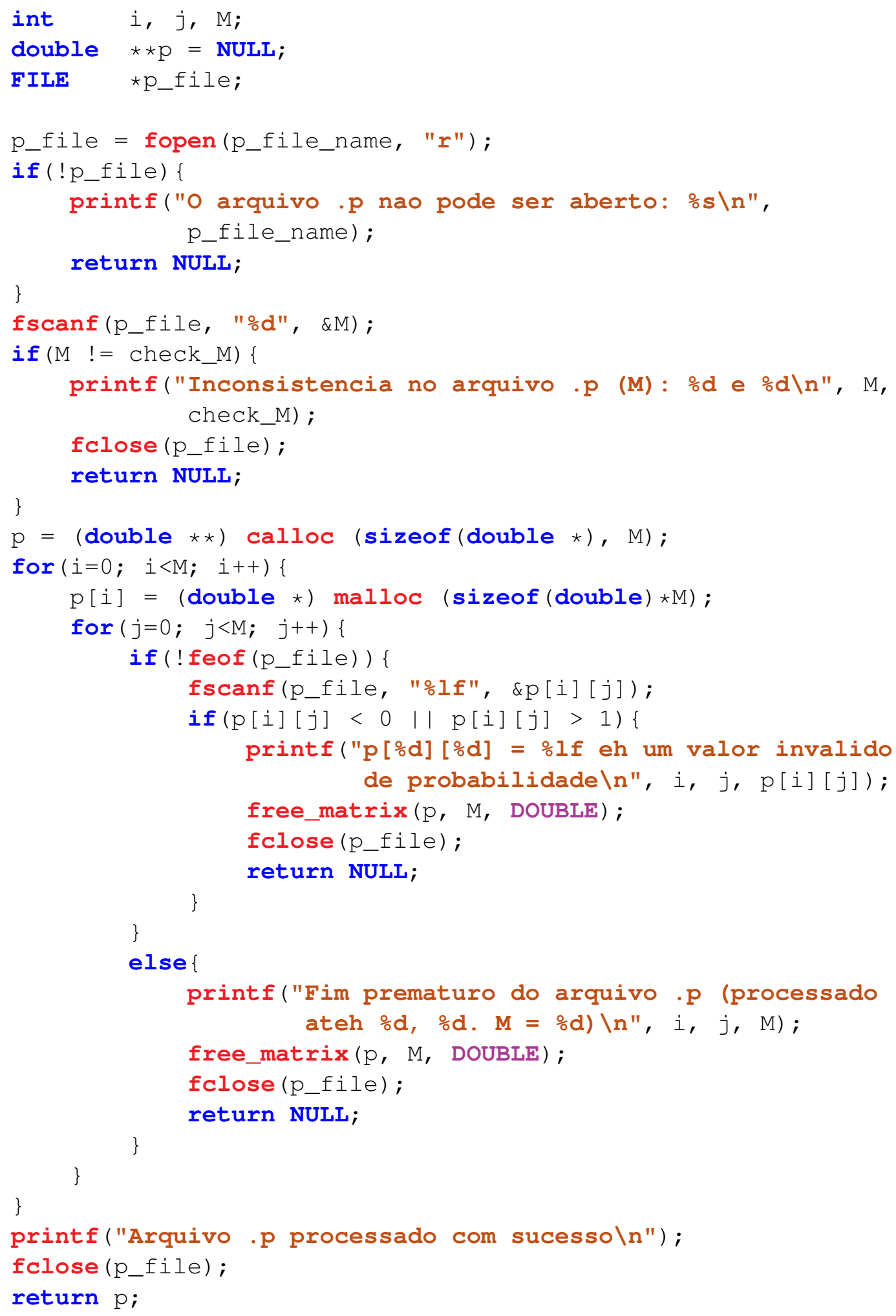




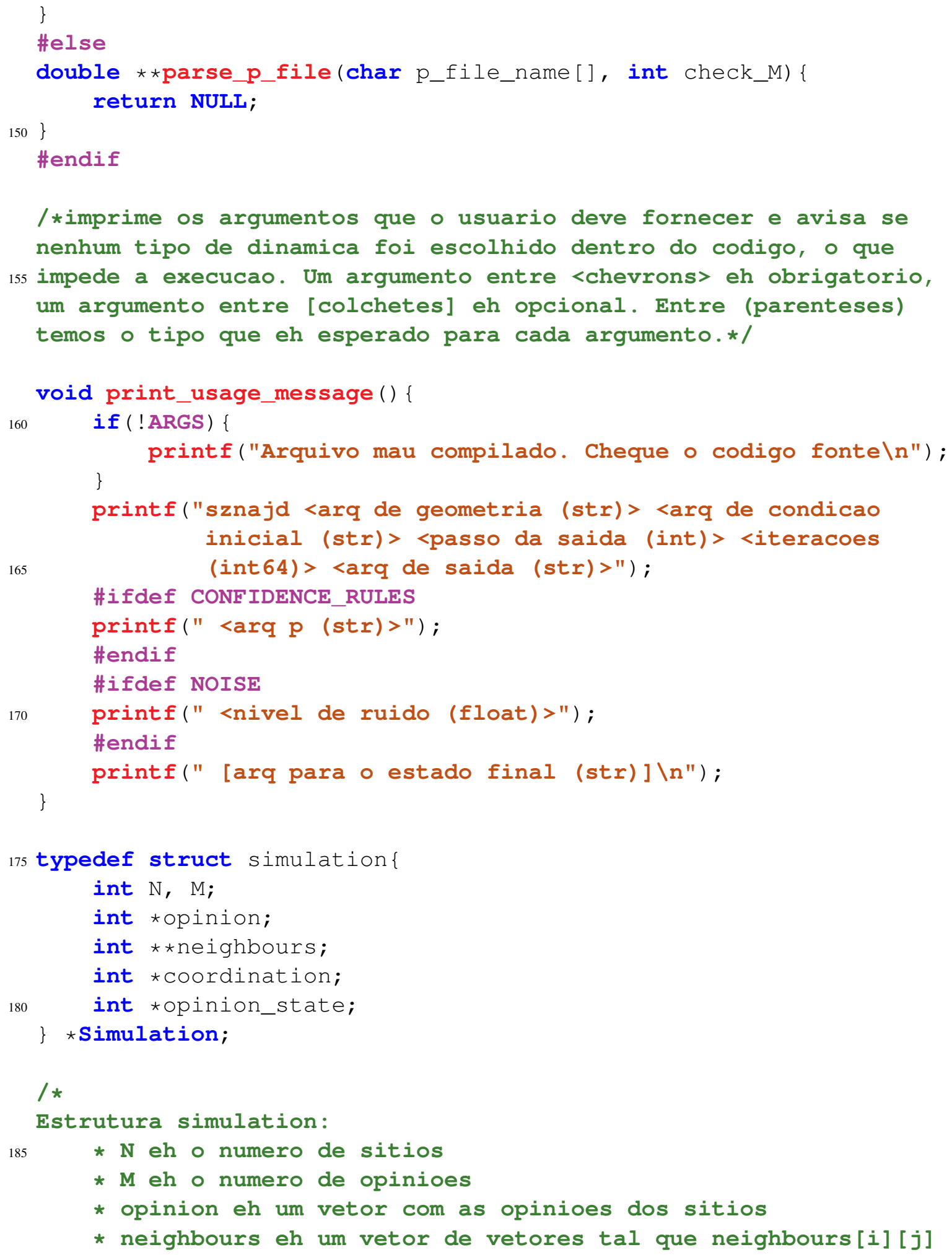


eh o j-esimo vizinho de $i$

190

* coordinations eh um vetor com os numeros de vizinhos

* opinion_state eh um vetor com o estado macroscopico, ou seja, o numero de sitios cm cada opiniao

o papel da estrutura eh basicamente o de um container

$195 * /$

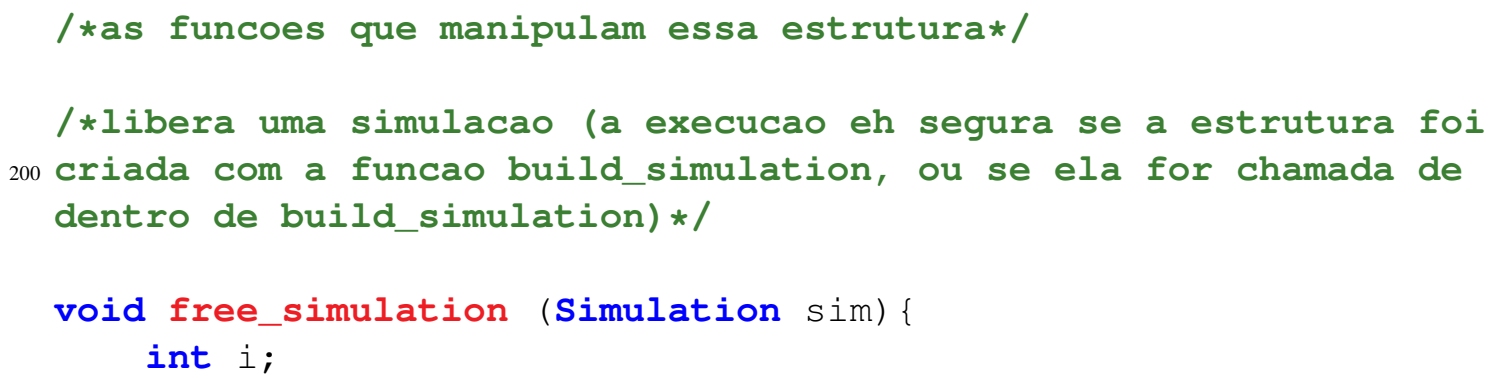

/*abre um arquivo de geometria e um de condicao inicial e inicia a 225 simulacao de acordo*/

Simulation build_simulation (char geometry_name[], char initial_condition_name [ ] ) \{

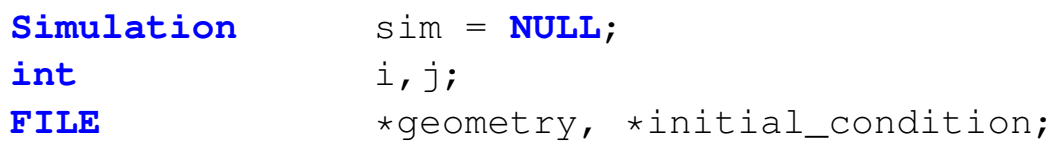




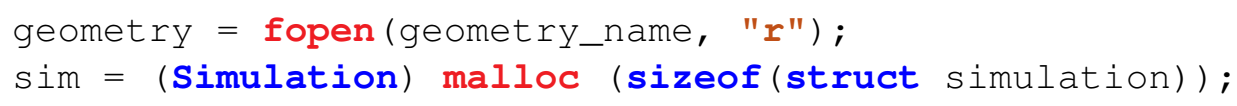
sim->opinion = sim->coordination = sim->opinion_state = NULL; sim->neighbours = NULL; 


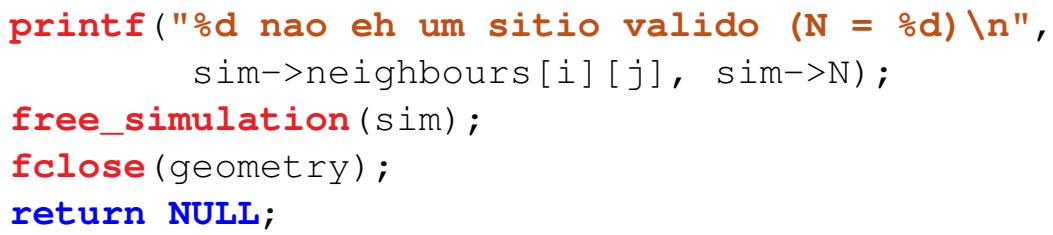




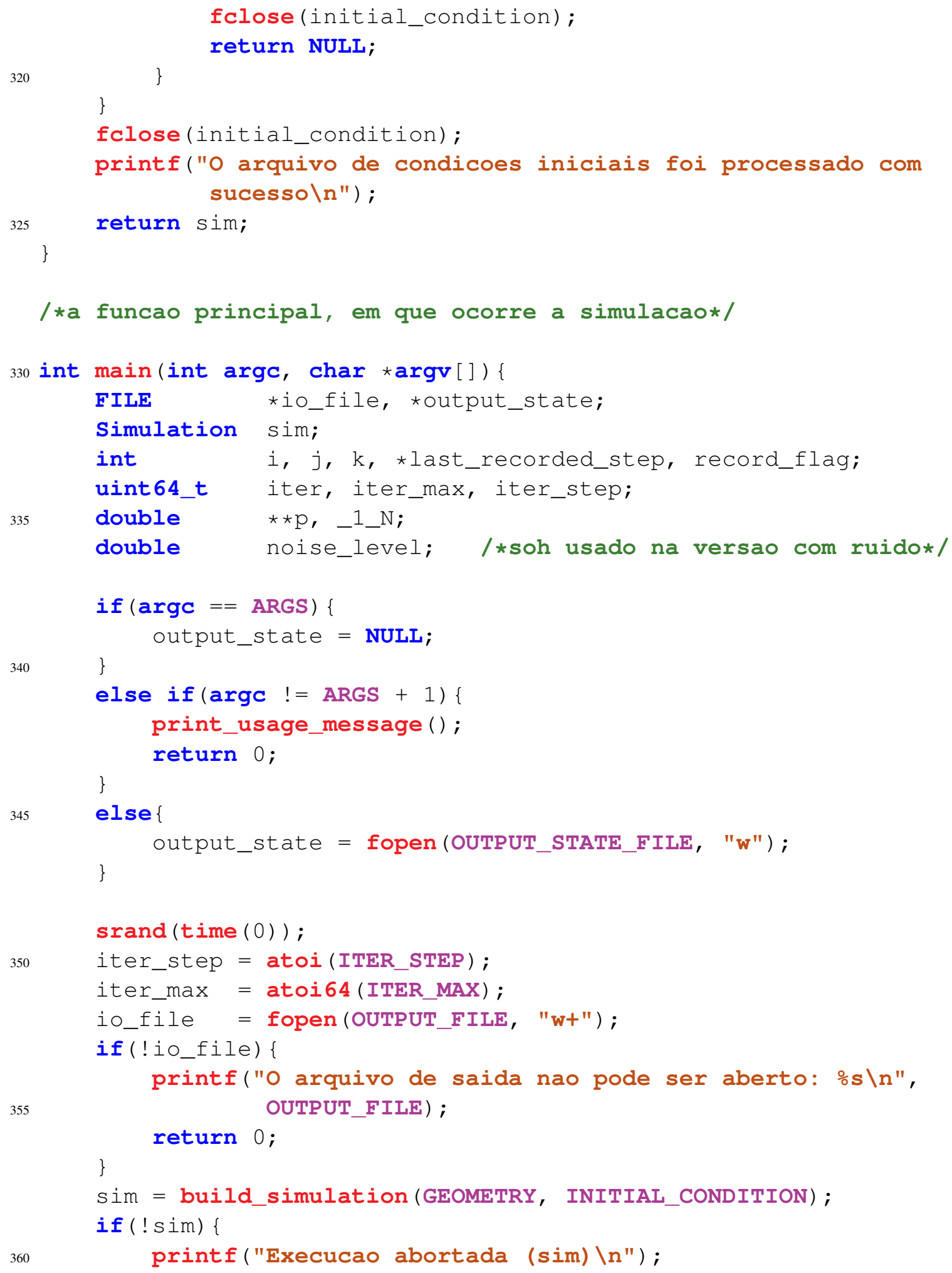




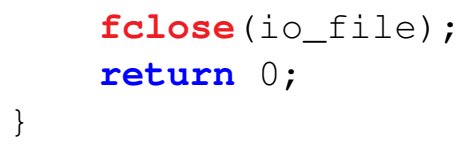


405

410

415

420

425

430

445

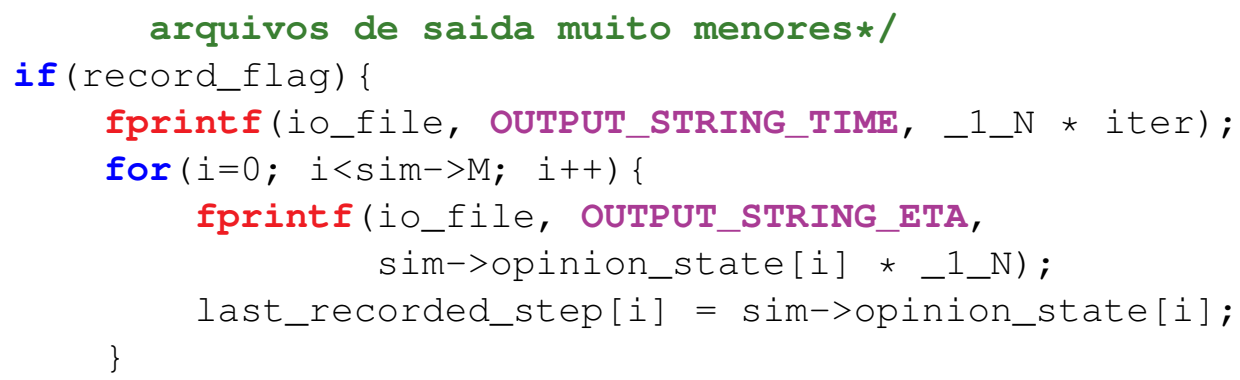

arquivos de saida muito menores*/ if (record_flag) \{

fprintf(io_file, OUTPUT_STRING_TIME, _1_N * iter); for $(i=0 ; \quad i<$ Sim->M; $i++)\{$

fprintf(io_file, OUTPUT_STRING_ETA, sim->opinion_state[i] * I $^{1}$ N) ;

last_recorded_step[i] = sim->opinion_state[i]; \}

fprintf(io_file, "\n");

\}

\}

$i=$ rand_aux $($ sim- $-\mathrm{N})$;

\#if defined(NOISE)

if ( ( double) rand ()) /RAND_MAX < noise_level) \{

sim->opinion_state[sim->opinion[i]] --;

sim->opinion[i] = rand_aux $($ sim- $>$ M);

sim->opinion_state[sim->opinion[i]] ++;

continue;

\#endif

$j=\operatorname{sim}->$ neighbours[i][rand_aux(sim->coordination[i])]; if (sim->opinion[i] == sim->opinion[j]) \{

$k=\operatorname{sim}->$ neighbours $[j][$ rand_aux $(\operatorname{sim}->\operatorname{coordination}[j])]$;

\#if defined(CONFIDENCE_RULES)

if ( ( double) rand ( ) ) /RAND_MAX <

p[sim->opinion[k]][sim->opinion[i]]) \{

/* regras de confianca */

\#elif defined(USUAL_SZNAJD)

if (sim->opinion[k] != sim->opinion[i])\{

/* modelo usual */

\#elif defined(BOUNDED_CONFIDENCE)

if (abs (sim->opinion[k] - sim->opinion[i]) ==1)\{ /* confianca limitada */

\#endif

$$
\begin{aligned}
& \text { sim->opinion_state[sim->opinion[i]] ++; } \\
& \text { sim->opinion_state[sim->opinion[k] }--; \\
& \text { sim->opinion[k] = sim->opinion[i]; }
\end{aligned}
$$


\}

fprintf(io_file, OUTPUT_STRING_TIME, _1_N * iter);

for $(i=0 ; i<$ Sim->M; $i++)\{$

450 fprintf(io_file, OUTPUT_STRING_ETA, sim->opinion_state[i]

$$
\text { * } \left.{ }^{1} \_\mathrm{N}\right) \text {; }
$$

\}

fprintf(io_file, "\n");

fclose (io_file);

455

printf("Simulacao concluida \n");

if (output_state) \{

fprintf(output_state, "\%d \n", sim->M) ;

for $(i=0 ; i<\operatorname{sim}->N$; $i++)\{$

fprintf(output_state, "\%d ", sim->opinion[i]);

460 \}

fflush (output_state);

fclose (output_state);

free_matrix (p, sim->M, DOUBLE);

465

free_simulation (sim);

return 0 ;

\section{B.2.1 Adaptações para o modelo do votante}

Para fazer a simulação do modelo do votante ao invés do modelo Sznajd é necesário trocar o código nas linhas 464 a 485 por

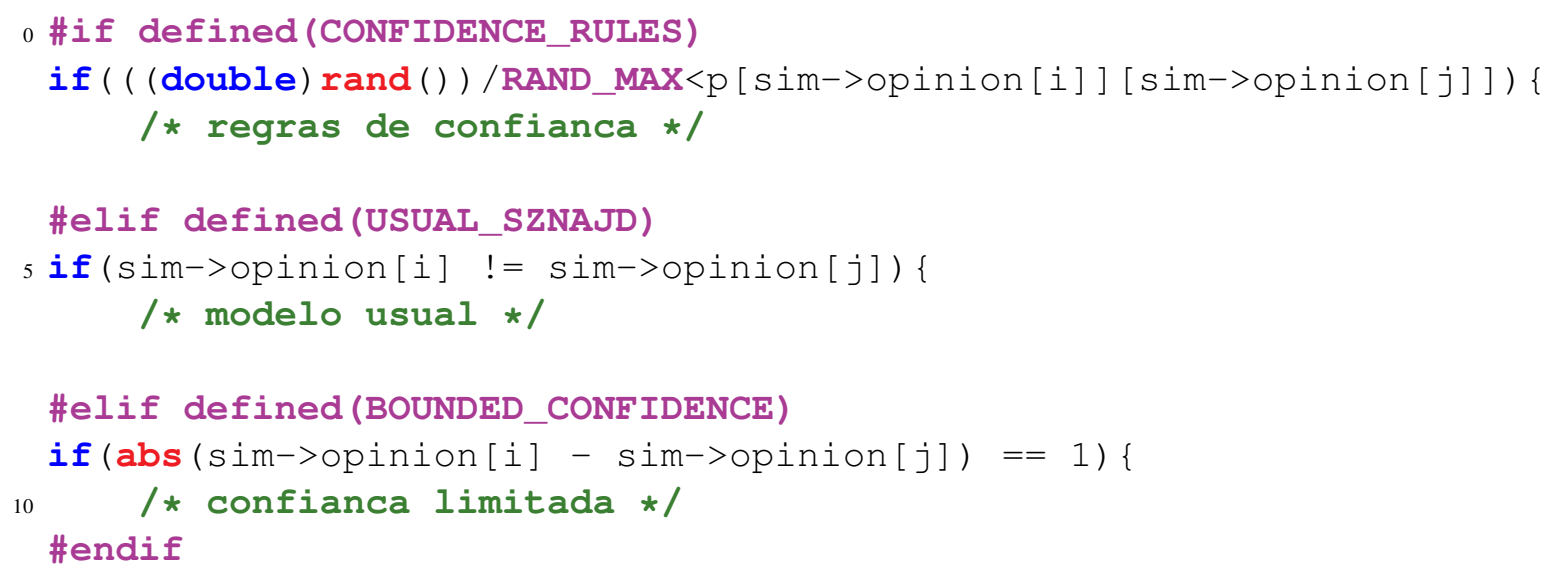




\section{B.3 cria_BA.c}

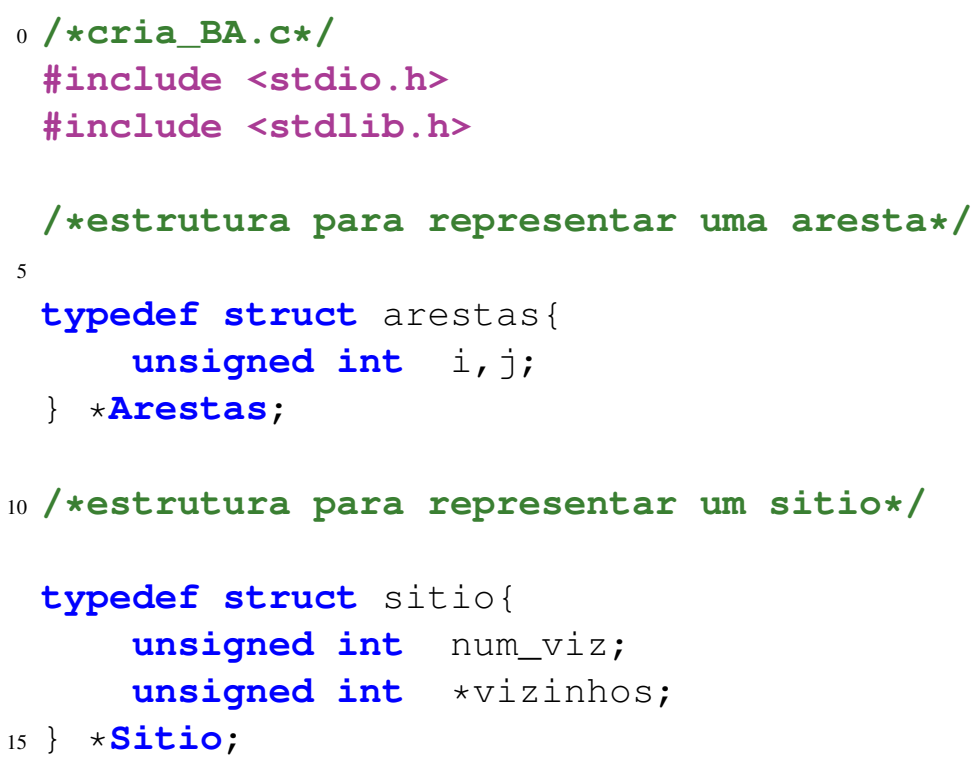




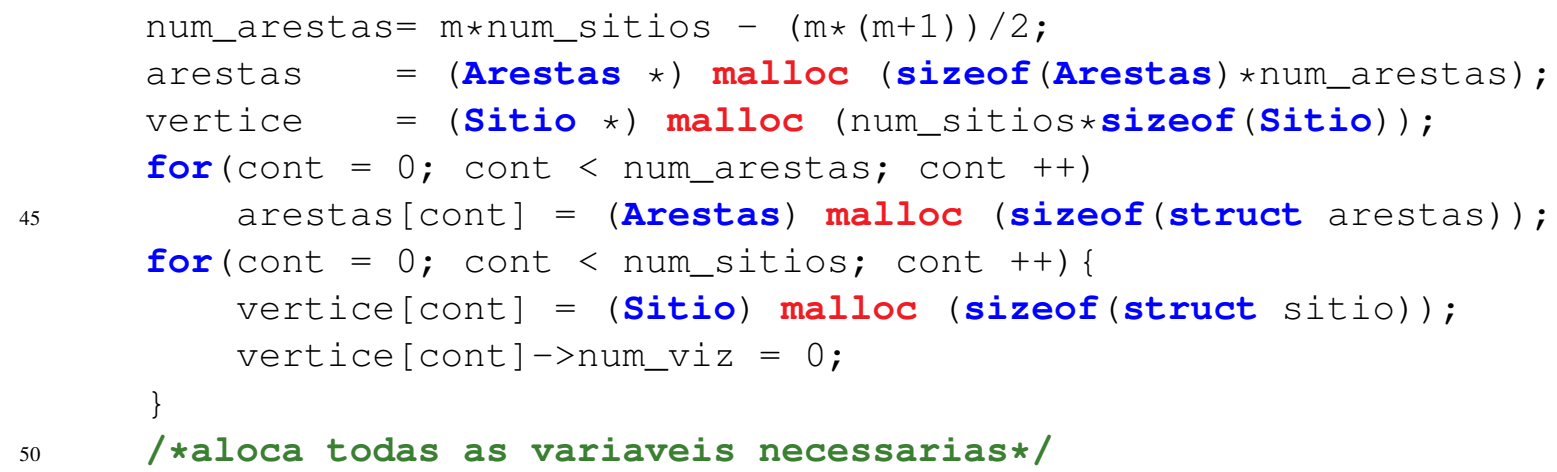
arestas [cont] = (Arestas) malloc (sizeof (struct arestas)); 
85

90

95

100

105

110

115

125

120 if (arestas [cont2]->i == sitio_sorteado || arestas[cont2]->j== sitio_sorteado)

break;

/*ou seja se alguma das arestas ja for entre j

e o sitio sorteado, temos uma repeticao*/

\}

if (cont2 != aresta_atual)

continue;

/ tou seja se ele saiu via break no loop, o que indica

repeticao, repita os sorteios para essa aresta*/

random /=2;

if $($ random\%2 $=0$ ) \{

arestas [aresta_atual] $->i=j$;

arestas[aresta_atual] $->j=$ sitio_sorteado;

\}

else \{

arestas[aresta_atual]->i = sitio_sorteado; arestas[aresta_atual] $->j=j$;

\}

/*coloca a aresta removendo possiveis vieses*/ cont ++ ;

aresta_atual ++ ;

\}

/*as arestas estao criadas*/

for (cont $=0 ;$ cont $<$ num_arestas; cont ++$)\{$

vertice [arestas [cont]->i]->num_viz ++;

vertice [arestas [cont]->j]->num_viz ++;

\}

for (cont $=0 ;$ cont $<$ num_sitios; cont ++$)\{$

vertice [cont]->vizinhos $=$

(int *) malloc ((vertice[cont]->num_viz) *sizeof(int)); vertice [cont]->num_viz $=0$;

/*essa variavel vai funcionar como um contador adiante*/

\}

/*aloca de forma correta os vetores de vizinhos*/

for (cont $=0 ;$ cont $<$ num_arestas; cont ++$)\{$

$\operatorname{aux}=$ vertice [arestas [cont] $->i]$;

aux->vizinhos [(aux->num_viz) ++] = arestas[cont]->j;

aux2 = vertice[arestas [cont]->j];

aux2->vizinhos [(aux2->num_viz) ++] = arestas[cont]->i;

\} 
/*atribui de forma correta os vizinhos de cada sitio*/

fprintf( $f, "$ od $\backslash \mathrm{n} "$, num_sitios);

for $($ cont $=0$; cont $<$ num_sitios; cont ++$)\{$

130

135

140

145

155

165

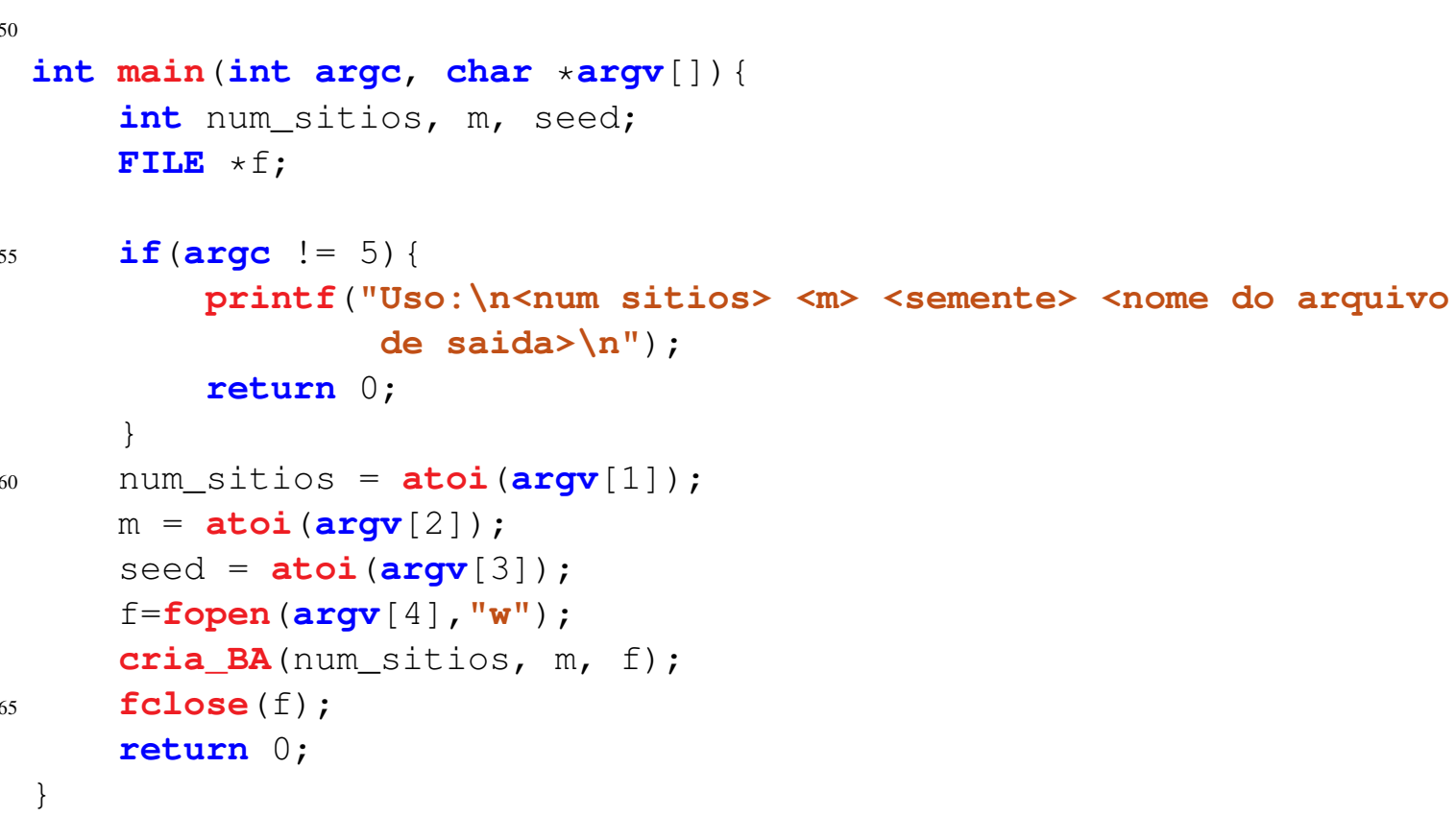




\section{Referências Bibliográficas}

[1] AsCH, S. E. Effects of group pressure upon the modification and distortion of judgment. In Groups, leadership and men., H. Guetzkow, Ed. Carnegie Press, Pittsburgh, PA, 1951. 2, 131

[2] AxELRod, R. The evolution of strategies in the iterated prisoner's dilemma. In $G e$ netic algorithms and simulated annealing, L. D. Davis, Ed. Morgan Kaufmann, 1987, pp. 32-41. 2

[3] Axelrod, R. The dissemination of culture: A model with local convergence and global polarization. The Journal of Conflict Resolution 41, 2 (Apr. 1997), 203-226. 31

[4] BARABÁsi, A. L., AND Albert, R. Emergence of scaling in random networks. Science 286, 5439 (1999), 509-512. 17

[5] Barabási, A. L., And Albert, R. Statistical mechanics of complex networks. Reviews of Modern Physics 74 (2002), 47-97. 2, 14, 16

[6] BeherA, L., AND Schweitzer, F. On spatial consensus formation: Is the sznajd model different from a voter model? International Journal of Modern Physics C 14, 10 (2003), 1331-1354. 28

[7] Bernardes, A. T., Stauffer, D., And Kertész, J. Election results and the sznajd model on barabasi network. European Physical Journal B 25 (2002), 123-127. 28

[8] Castellano, C., Fortunato, S., And Loreto, V. Statistical physics of social dynamics. Reviews of Modern Physics 81 (2009), 591-646. 1

[9] Cláudio Castellano, R. P.-S., And nOz, M. A. M. Nonlinear q-voter model. Physical Review E 80, 4 (2009), 041129. 29

[10] Costa-Filho, R. N., Almeida, M. P., Andrade, J. S., And Moreira, J. Scaling behaviour in a proportional voting process. Physical Review E 60 (1999), 10671068. 28 
[11] Deffunant, G., Neau, D., Amblard, F., And Weisbuch, G. Mixing beliefs among interacting agents. Advances in Complex Systems 3 (2000), 87-98. 30

[12] Do Prado, C. P. C., ANd Fiedler-FerRara, N. Caos - Uma Introdução. Edgard Blücher, 1994. 18

[13] Elster, J. Sour Grapes: Studies in the subvertion of rationality. Cambridge University Press, 1985. 2

[14] Festinger, L. A theory of Cognitive Dissonance. Stanford University Press, 1957. 2

[15] Fortunato, S. On the consensus threshold for the opinion dynamics of krausehegselmann. International Journal of Modern Physics C 16, 2 (2005), 259-270. 31

[16] Frachebourg, L., And Krapivsky, P. L. Exact results for kinetics of catalytic reactions. Physical Review E 53, 4 (Apr. 1996), 3009. 27

[17] Galam, S. Local dynamics vs. social mechanisms: A unifying frame. Europhysics Letters 70 (2005), 705-711. 25

[18] Galam, S., Gefen, Y., And Shapir, Y. Sociophysics: A mean behavior model for the process of strike. The Journal of Mathematical Sociology 9 (1982), 1-13. 2

[19] Granovetter, M. S. The strenght of weak ties. American Journal of Sociology 78, 6 (1973), 1360-1380. 138

[20] Granovetter, M. S. The strenght of weak ties: A network theory revisited. Sociological Theory 1 (1983), 201-233. 138

[21] Hegselmann, R., And Krause, U. Opinion dynamics and bounded confidence models, analysis, and simulation. Journal of Artificial Societies and Social Simulation 5, 3 (2002). 30

[22] Holley, R. A., AND Liggett, T. M. Ergodic theorems for weakly interacting infinite systems and the voter model. Annals of Probability 3, 4 (1975), 643-663. 25

[23] Johnson, C. R. Suficient conditions for d-stability. Journal of Economic Theory 9 (1974), 53-62. 148

[24] KAHn, A. B. Topological sorting of large networks. Communications of the ACM 5, 11 (1962), 558-562. 140

[25] Katarzina Sznajd-Weron, M. T., And Timpanaro, A. M. Phase transition in the sznajd model with independence. epl 96 (2011), 48002. 138 
[26] Klemm, K., Eguíluz, V. M., Toral, R., And Miguel, M. S. Nonequilibrium transitions in complex networks: A model of social interaction. Physical Review E 67 (Feb. 2003), 026120. 31

[27] Kondrat, G., And Sznajd-Weron, K. Three types of outflow dynamics on square and triangular lattices and universal scaling. Physical Review E 77 (2008), 021127. 25

[28] LEE, S. W. S., AND SCHWARZ, N. Washing away postdecisional dissonance. Science 328, 5979 (2010), 709. 2

[29] Masoller, C., Schifino, A. S., And Romanellic, L. Characterization of strange attractors of lorenz model of general circulation of the atmosphere. Chaos, Solitons and Fractals 6 (1995), 357-366. 22

[30] OCHRombel, R. Simulation of sznajd sociophysics model with convincing single opinions. International Journal of Modern Physics C 12, 7 (July 2001), 1091. 27

[31] PRICE, D. D. S. A general theory of bibliometric and other cumulative advantage processes. Journal of the American Society for Information Science and Technology 27, 5 (Sept. 1976), 292-306. 17

[32] Reichenbach, T., Mobilia, M., And Frey, E. Mobility promotes and jeopardizes biodiversity in rock-paper-scissors games. Nature 448 (2007), 1046-1049. 128

[33] Schelling, T. C. Dynamic models of segragation. The Journal of Mathematical Sociology 1 (1971), 143-186. 2

[34] Schulze, C. Sznajd opinion dynamics with global and local neighbourhood. International Journal of Modern Physics C 15, 6 (July 2004), 867-872. 29

[35] SCIPY. http://www.scipy.org. 157

[36] ShERIF, M. A study of some social factors in perception. Master's thesis, Columbia University, 1935. 2, 131

[37] Sousa, A. O., And SÁnchez, J. R. Outward-inward information flux in an opinion formation model on different topologies. Physica A 361, 1 (Feb. 2006), 319-328. 25

[38] Stauffer, D. The sznajd model of consensus building with limited persuasion. International Journal of Modern Physics C 13, 3 (2002), 315-318. 29

[39] Stauffer, D., Sousa, A. O., And de Oliveira, S. M. Generalization to square lattice of sznajd sociophysics model. International Journal of Modern Physics C 11 (2000), 1239-1245. 28 
[40] Sznajd-Weron, K., AND SznaJd, J. Opinion evolution in closed community. International Journal of Modern Physics C 11, 6 (2000), 1157-1165. 28

[41] Tainaka, K., And Itoh, Y. Topological phase transition in biological ecosystems. Europhysics Letters 15 (1991), 399-404. 136

[42] TARJAN, R. E. Edge-disjoint spanning trees and depth-first search. Algorithmica 6, 2 (1976), 171-185. 140

[43] Timpanaro, A. M., And do Prado, C. P. C. A generalized sznajd model. Physical Review E 80, 2 (2009), 021119. 5, 6, 24, 28, 117, 138

[44] Timpanaro, A. M., And do Prado, C. P. C. Coexistence of interacting opinions in a generalized sznajd model. Physical Review E 84, 2 (2011), 027101. 138

[45] Timpanaro, A. M., And do Prado, C. P. C. Connections between the sznajd model with general confidence rules and graph theory. arxiv:1205.3008 (2012). 138

[46] VAnnucchi, F. S. Sznajd model in complex networks (o modelo sznajd em redes complexas). Master's thesis, Universidade de São Paulo - São Paulo, Brazil, 2006. 6, 28

[47] Watts, D. J., And Strogatz, S. H. Collective dynamics of 'small-world' networks. Nature 393, 6684 (June 1998), 440-442. 16

[48] WeIDLICH, W. The statistical description of polarization phenomena in society. British Journal of Mathematical and Statistical Psychology 24 (1971), 251-266. 2 